\title{
O problema de Riemann-Hilbert para campos vetoriais complexos
}



Data de Depósito:

Assinatura:

\title{
Camilo Campana
}

\section{O problema de Riemann-Hilbert para campos vetoriais complexos}

\begin{abstract}
Tese apresentada ao Instituto de Ciências Matemáticas e de Computação - ICMC-USP, como parte dos requisitos para obtenção do título de Doutor em Ciências - Matemática. VERSÃO REVISADA
\end{abstract}

Área de Concentração: Matemática

Orientador: Prof.

Dr. Adalberto

Panobianco Bergamasco

Coorientador: Prof. Dr. Abdelhamid Meziani 
Ficha catalográfica elaborada pela Biblioteca Prof. Achille Bassi e Seção Técnica de Informática, ICMC/USP, com os dados fornecidos pelo(a) autor(a)

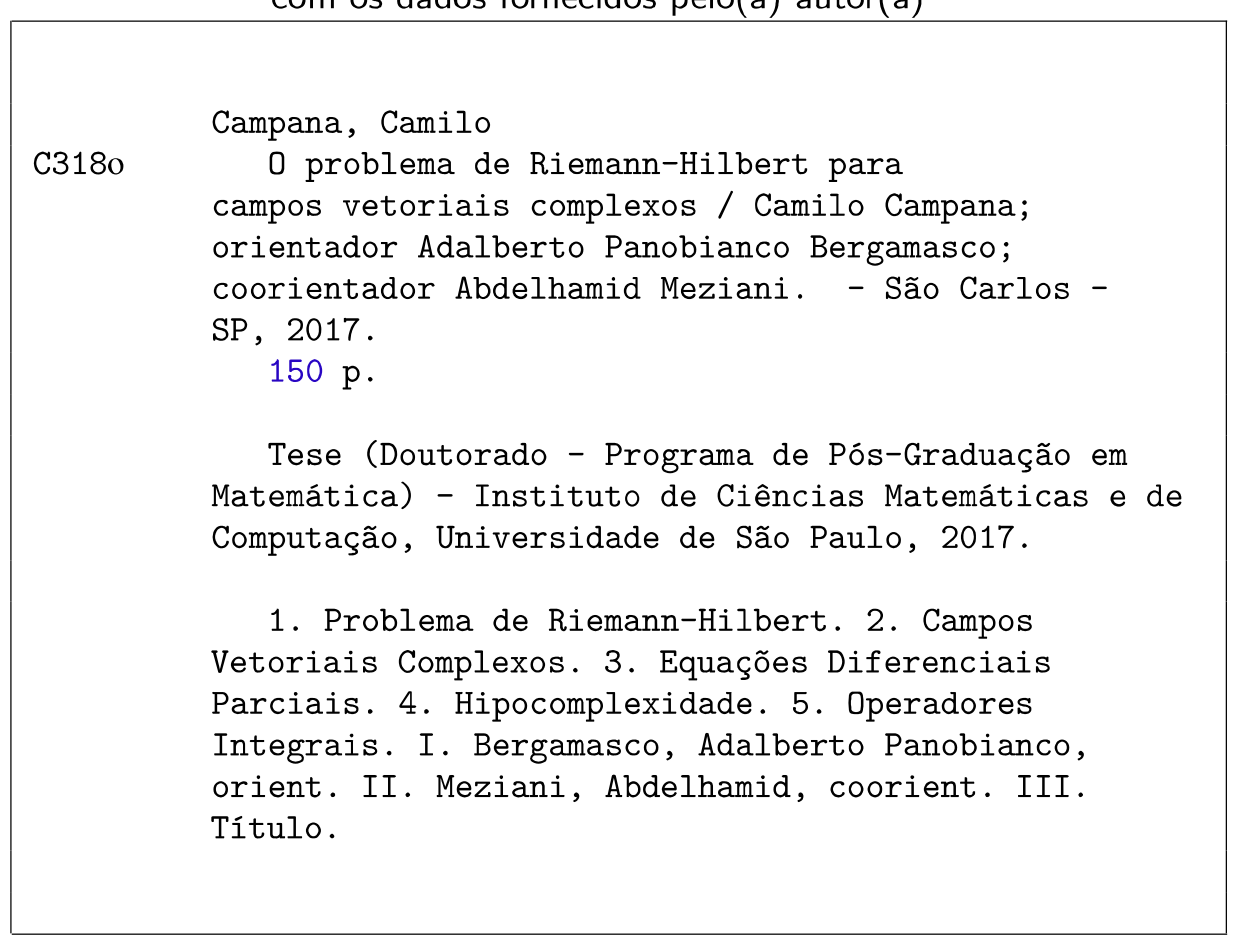




\section{Camilo Campana}

\section{The Riemann-Hilbert problem for complex vector fields}

Doctoral dissertation submitted to the Instituto de Ciências Matemáticas e de Computação - ICMCUSP, in partial fulfillment of the requirements for the degree of the Doctorate Program in Mathematics. FINAL VERSION

Concentration Area: Mathematics

Advisor: Prof. Dr. Adalberto Panobianco Bergamasco

Co-advisor: Prof. Dr. Abdelhamid Meziani 

Dedico este trabalho a minha família.

Em especial a minha bisavó Maria. 

A Deus.

Ao programa de pós graduação do ICMC-USP (Instituto de Ciências Matemáticas e de Computação). Aos professores e funcionários que contribuíram para a confecção dessa dissertação.

Agradecimento especial ao Professor Adalberto Panobianco Bergamasco, meu orientador de doutorado, pela amizade, direcionamento e dedicação.

Ao meu coorientador, Professor Abdelhamid Meziani, pelo trabalho engajado, dedicação, disponibilidade e amizade.

Ao Professor Paulo Leandro Dattori da Silva pela grande ajuda, não apenas com a matemática, pela amizade e pelo trabalho conjunto.

A minha família, especialmente, meus pais Lourdes e Judçon. Meus avós, Jovelina e Estevaldo e aos meus bisavós Maria e Américo.

Aos meus amigos. Em especial, Laís, Dione e Rodrigo.

Aos professores da UFES que foram de grande importância no início dessa jornada na matemática.

Gostaria de destacar a importância do estágio no exterior, apoiado pela FAPESP (processo n 2013/26463 - 7, Fundação de Amparo à Pesquisa do Estado de São Paulo (FAPESP)), para realização deste trabalho. Agradeço ao Professor Abdelhamid Meziani pela supervisão durante o período do estágio e a Florida International University por contribuir com o ambiente e estrutura para sua realização.

A todos os citados e às pessoas que não mencionei, mas que fizeram parte da minha trajetória, fica registrado o meu agradecimento.

À CAPES pelo apoio financeiro nos meses iniciais do doutorado.

À FAPESP (processo nº 2013/08452 - 8, Fundação de Amparo à Pesquisa do Estado de São Paulo (FAPESP)) pelo apoio financeiro. 

"É melhor acender uma vela do que praguejar contra a escuridão." (Provérbio popular) 



\section{RESUMO}

CAMPANA, C.. O problema de Riemann-Hilbert para campos vetoriais complexos. 2017. 150 f. Tese (Doutorado em Ciências - Matemática) - Instituto de Ciências Matemáticas e de Computação (ICMC/USP), São Carlos - SP.

Este trabalho trata de problemas de contorno definidos no plano. O problema central desta tese é o chamado Problema de Riemann-Hilbert, o qual pode ser descrito como segue. Seja $L$ um campo vetorial complexo não singular definido em uma vizinhança do fecho de um aberto simplesmente conexo do plano com fronteira suave.

O Problema de Riemann-Hilbert para o campo $L$ consiste em obter uma solução para a equação $L u=F(x, y, u)$ no aberto em estudo, sendo dada uma função $F$ mensurável. Pede-se também que a solução tenha extensão contínua até a fronteira e que satisfaça lá uma condição adicional; trabalha-se aqui no contexto das funções Hölder contínuas.

Foram obtidos resultados para o problema acima no caso em que $L$ pertence a uma classe de campos hipocomplexos.

O caso clássico conhecido é quando o campo vetorial é o operador de Cauchy-Riemann, ou, mais geralmente, quando é um campo elítico.

Palavras-chave: Problema de Riemann-Hilbert, Campos Vetoriais Complexos, Equações Diferenciais Parciais, Hipocomplexidade, Operadores Integrais. 



\section{ABSTRACT}

CAMPANA, C.. The Riemann-Hilbert problem for complex vector fields. 2017. $150 \mathrm{f}$. Tese (Doutorado em Ciências - Matemática) - Instituto de Ciências Matemáticas e de Computação (ICMC/USP), São Carlos - SP.

This work deals with boundary problems in the plane. The central problem in this thesis is the so-called Riemann-Hilbert problem, which may be described as follows. Let $L$ be a non-singular complex vector field defined on a neighborhood of the closure of a simply connected open subset of the plane having smooth boundary.

The Riemann-Hilbert problem for the vector field $L$ consists in finding a solution to the equation $L u=F(x, y, u)$ on the open set under study, where the given function $F$ is measurable. It is also required that the solution have a continuous extension up to the boundary and satisfy an additional condition there.

Results were obtained for the above problem when $L$ belongs to a class of hypocomplex vector fields.

The well-known classical case is the one in which the vector field under study is the CauchyRiemann operator, or more generally when it is an elliptic vector field.

Key-words: Riemann-Hilbert Problem, Complex Vector Fields, Partial Differential Equations, Hipocomplexity, Integral Operators. 

$1.1 \quad 0$ problema clássico de Riemann-Hilbert . . . . . . . . . . . 17

$1.2 \quad 0$ problema de Riemann-Hilbert para um campo vetorial complexo $L \mathbf{1 8}$

1.3 Descrição do conteúdo da tese . . . . . . . . . . . . 19

PRELIMINARES . . . . . . . . . . . . . . . . . 25

$2.1 \quad$ Espaço de funções . . . . . . . . . . . . . . . . . 25

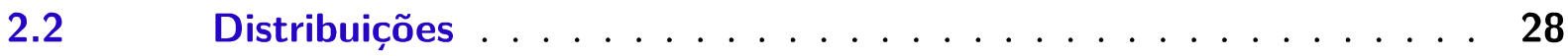

2.3 Campos vetoriais . . . . . . . . . . . . . . 29

2.3.1 Campos vetoriais de tipo finito e infinito . . . . . . . . . . 37

$2.4 \quad 0$ índice . . . . . . . . . . . . . . . . 38

2.5 Transversalidade de uma curva em relação a campo . . . . . . . 44

3 A CLASSE DOS CAMPOS VETORIAIS EM ESTUDO . . . . . 47

$3.1 \quad$ Uma classe de campos hipocomplexos . . . . . . . . . . . 47

$3.2 \quad$ Normalização . . . . . . . . . . . . . . . . . 53

3.3 Observações acerca do caso hipoelítico . . . . . . . . . 56



$5 \quad$ A EQUAÇÃO $L u=f \ldots \ldots \ldots \ldots \ldots$. . . . . . . . . . . . . .

$5.1 \quad 0$ operador integral $T_{Z} \ldots \ldots \ldots \ldots 71$

$5.2 \quad$ Existência de solução para a equação $L u=f \ldots \ldots$. . . . . . . . 76

$5.3 \quad$ Continuidade Hölder das soluções de $L u=f \ldots \ldots$. . . . . . . . . 80

$5.4 \quad$ Equação semilinear $L u=F(x, y, u) \ldots \ldots \ldots \ldots$

$5.5 \quad$ Princípio da Similaridade . . . . . . . . . . . . . . . 89

5.5.1 Lemas de diferenciação . . . . . . . . . . . . . . . . . . 90

5.5.2 Princípio da Similaridade para $L \ldots \ldots \ldots$. . . . . . . . . 95

6 O PROBLEMA DE RIEMANN-HILBERT . . . . . . . . . . . 99

$6.1 \quad 0$ operador integral $T_{\mathscr{Z}}^{\kappa} \ldots \ldots \ldots \ldots \ldots$

$6.2 \quad 0$ operador $S_{\mathscr{Z}} \ldots \ldots \ldots \ldots$. . . . . . . . . . . . . . .

6.3 O problema de Riemann-Hilbert para equações do tipo $L u=F(x, y, u) \mathbf{1 0 9}$

$6.4 \quad$ Uma propriedade do operador $T_{\mathscr{Z}} \ldots \ldots \ldots$ 
6.5 0 problema de Riemann-Hilbert para a equação $L u=a u+b \bar{u}+f \ldots 118$

6.6 0 problema de Riemann-Hilbert para a equação $L u=a u+f$ quando $L$ é hipoelítico . . . . . . . . . . . . . . . . . . . . . . 125

6.6.1 Resolubilidade global para campos hipoelíticos no plano . . . . . . 125

6.6.2 O problema de Riemann-Hilbert para a equação $L u=a u+f$. . . . 128

ANEXO A TEOREMAS IMPORTANTES USADOS NO TEXTO 131

ANEXO B ALGUNS RESULTADOS DA TEORIA CLÁSSICA . . . 135

REFERÊNCIAS . . . . . . . . . . . . . . . . . . 145

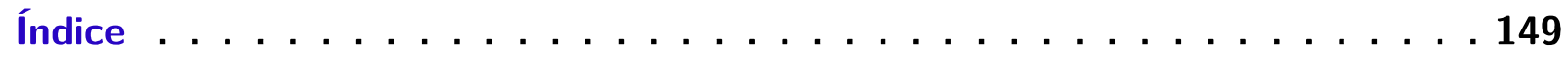


A área de interesse da presente tese refere-se ao estudo de problemas de contorno para campos vetoriais complexos.

A resolubilidade local de sistemas involutivos de equações diferenciais de primeira ordem (sem condições adicionais, tais como de Cauchy, Dirichlet, entre outras) tem experimentado muitos avanços (ver (BERHANU; CORDARO; HOUNIE, 2008) e (TREVES, 1992)). Um próximo passo natural é o estudo de problemas de contorno, como por exemplo, os problemas de Riemann-Hilbert associados a um campo vetorial complexo no plano.

\subsection{O problema clássico de Riemann-Hilbert}

O problema clássico de Riemann-Hilbert é um problema importante da teoria de problemas de contorno para funções holomorfas (analíticas). O problema é como segue. Dados um aberto simplesmente conexo $D \subset \mathbb{C}$ e funções $\Lambda, \phi$ definidas na fronteira $\partial D$ com $\Lambda$ a valores complexos, sem zeros e $\phi$ a valores reais, obter uma função holomorfa $h$ em $D$, contínua em $\bar{D}$, tal que $\mathfrak{R}(\bar{\Lambda} h)=\phi$ em $\partial D$. Em outras palavras, escrevendo em termos do operador de Cauchy-Riemann, $\partial / \partial \bar{z}$, temos o problema de contorno

$$
\left\{\begin{array}{lll}
\frac{\partial h}{\partial \bar{z}}=0 & \text { em } & D \\
\Re(\bar{\Lambda} h)=\phi & \text { em } & \partial D .
\end{array}\right.
$$

Este problema, bem como sua generalização ao caso em que a busca de uma função analítica é substituída pela busca de uma solução de uma equação elítica (linear ou não linear), tem sido estudado extensivamente sob várias hipóteses envolvendo a fronteira e os dados $\Lambda$ e $\phi$. Suas aplicações a problemas em física e engenharia são numerosas e bem documentadas (ver (ABLOWITZ; CLARKSON, 1991), (BEGEHR; CELEBI; TUTSCHKE, 1999), (BEGEHR; GILBERT; WEN, 1999), (CHEN, 1993), (MUSKHELISHVILI, 1953), (VEKUA, 1962) parte 2, por exemplo). 
Para um pequeno apanhado histórico veja (GAKHOV, 1966) e (MUSKHELISHVILI, 1953).

\subsection{0 problema de Riemann-Hilbert para um campo ve- torial complexo $L$}

Seja $L$ um campo vetorial complexo não singular de classe $C^{\varepsilon}, 0<\varepsilon<1$, definido em uma vizinhança de um aberto simplesmente conexo $\Omega \subset \mathbb{R}^{2}$ com fronteira suave e sejam $\Lambda \in C^{\alpha}(\partial \Omega ; \mathbb{C}),|\Lambda|=1, \phi \in C^{\alpha}(\partial \Omega ; \mathbb{R}), 0<\alpha<1$. O Problema de Riemann-Hilbert para o campo $L$ consiste em obter uma função contínua em $\bar{\Omega}$ que seja solução do problema de contorno

$$
\begin{cases}L u=0 & \text { em } \quad \Omega \\ \Re(\bar{\Lambda} u)=\phi & \text { em } \quad \partial \Omega\end{cases}
$$

ou mais geralmente quando consideramos, no lugar da equação $L u=0$ em $\Omega$ acima, uma equação da forma $L u=F(x, y, u)$ em $\Omega$, onde $F: \bar{\Omega} \times \mathbb{C} \rightarrow \mathbb{C}$ é uma função mensurável. Observemos que quando $L$ é elítico em $\bar{\Omega}$ (isto é, $L$ e seu conjugado complexo $\bar{L}$ são linearmente independentes em todo o conjunto $\bar{\Omega}$ ), então $L$ é equivalente ao operador de Cauchy-Riemann $\partial / \partial \bar{z}$ e o problema se reduz ao problema de Riemann-Hilbert clássico, que em muitos casos já foi resolvido. $O$ problema (1.2) difere do caso clássico quando o conjunto dos pontos não elíticos

$$
\Sigma=\left\{\mathrm{p} ; L_{\mathrm{p}} \text { e } \bar{L}_{\mathrm{p}} \text { são linearmente dependentes }\right\}
$$

chamado de conjunto característico de $L$, é não vazio. Neste caso, em que $\Sigma$ é não vazio, $L$ é chamado de campo elítico com degenerescências. No trabalho (MEZIANI, 2011), foi considerado o problema de Riemann-Hilbert para uma classe de campos vetoriais complexos. Pelo que sabemos, o problema de Riemann-Hilbert (1.2) para campos elíticos com degenerescências não foi considerado antes de (MEZIANI, 2011).

Uma das dificuldades do estudo do Problema de Riemann-Hilbert para um campo vetorial complexo $L$, definido num vizinhança de $\Omega$ como acima, é obter soluções contínuas para a equação $L u=f$ em $\Omega$, para $f \in L^{p}(\Omega), p>1$. Já a resolubilidade local para campos vetoriais complexos (de classe $C^{\infty}$ ) teve muitos avanços e é caracterizada pela condição $(\mathrm{P})$ de Nirenberg e Treves (veja (BERHANU; CORDARO; HOUNIE, 2008)).

Os resultados obtidos, que serão descritos na próxima seção, generalizam resultados de resolubilidade para o Problema de Riemann-Hilbert clássico.

Os principais resultados desta tese foram obtidos em trabalho conjunto e podem ser encontrados nos artigos (CAMPANA; SILVA; MEZIANI, 2017), (CAMPANA; SILVA; MEZIANI, 2016) e (CAMPANA; MEZIANI, 2016). 
Muitas das técnicas desenvolvidas nesta tese são generalizações de técnicas da teoria das Funções Analíticas Generalizadas, que podem ser encontradas nos livros (VEKUA, 1962), (BEGEHR, 1994) e (GAKHOV, 1966).

\subsection{Descrição do conteúdo da tese}

Agora vamos detalhar o conteúdo da tese.

O Capítulo 2 é dedicado a definições, conceitos básicos da área de Equações Diferenciais Parciais Lineares e alguns resultados que servem de base para o texto envolvendo campos hipocomplexos, como, por exemplo, a existência de integral primeira global que denotamos por $Z$. Também nesse capítulo introduzimos o índice de uma função com respeito a uma curva que será importante no estudo do problema de Riemann-Hilbert (Capítulo 6).

No Capítulo 3 estabelecemos uma classe de campos vetoriais complexos, definidos em um aberto $\tilde{\Omega}$ de $\mathbb{R}^{2}$, que chamaremos de classe $\mathscr{X}(\tilde{\Omega})$, ou apenas de classe $\mathscr{X}$. Esta classe pode ser caracterizada da seguinte forma. Se $L$ é um campo vetorial pertencente à classe $\mathscr{X}$ então $L$ é hipocomplexo (veja a definição 7 na página 35) e o conjunto característico $\Sigma$ de $L$ é uma subvariedade mergulhada de $\Omega$ de dimensão 1 , com um número finito de componentes conexas, denotadas por $\Sigma_{j}, j=1,2, . ., N$. Além disso, para cada componente conexa $\Sigma_{j}$ existe uma constante $\sigma_{j}>0$ tal que para cada $\mathrm{p} \in \Sigma_{j}$, existem um aberto $U, \mathrm{p} \in U \subset \tilde{\Omega}$, coordenadas locais $(s, t)$ centradas em $\mathrm{p}$ e uma função $\beta$ a valores reais tal que a função

$$
Z_{\sigma_{j}, \beta}(s, t)=s+i\left(\frac{t|t|^{\sigma_{j}}}{1+\sigma_{j}}+\beta(s)\right)
$$

é uma integral primeira de $L$.

Denotamos por $\sigma_{\mathrm{M}}=\max \left\{\sigma_{j} ; j=1,2, \ldots, N\right\}$.

Além de estabelecer a classe, é feito um estudo de normalização local e propriedades inerentes à classe. A normalização local é usada para fazer conexão entre duas partes deste texto, a parte Hard, formada por lemas técnicos (Capítulo 4), e a parte Soft, (situada nos Capítulos 5, 6 e 7) formada pela aplicação de resultados de Topologia e Análise Funcional.

O Capítulo 4 é composto por lemas técnicos que dão estimativas de integrais com parâmetros definidas em abertos de $\mathbb{R}^{2}$. Tais lemas servem de suporte para a boa definição de operadores integrais nos capítulos seguintes bem como estimativas envolvendo os mesmos.

No Capítulo 5 tratamos de equações envolvendo $L$. Em especial estudamos, para $L$ pertencente à classe $\mathscr{X}(\tilde{\Omega})$, a equação $L u=f \operatorname{em} \Omega$, com $\Omega$ um aberto pré-compacto tal que $\bar{\Omega} \subset \tilde{\Omega}$ e $f$ uma função em um espaço $L^{p}(\Omega)$ adequado. O caminho tomado, para tal, foi o estudo de operadores integrais relacionados ao campo $L$. Mais especificamente, dada uma integral 
primeira global $Z$ de $L$ no aberto $\Omega$, que é um homeomorfismo sobre sua imagem, definimos o operador integral $T_{Z}$, para $f \in L^{1}(\Omega)$, por

$$
T_{Z} f(x, y)=\frac{1}{2 \pi i} \int_{\Omega} \frac{f(\xi, \eta)}{Z(\xi, \eta)-Z(x, y)} d \xi d \eta, \text { para }(x, y) \in \bar{\Omega} .
$$

O operador integral $T_{Z}$ possui a seguinte propriedades: existe uma constante $M=$ $M\left(p, \sigma_{1}, \ldots, \sigma_{N}, \Omega\right)>0$ tal que se $f \in L^{p}(\Omega)$, com $p>2+\sigma_{\mathrm{M}}$, vale

$$
\left|T_{Z} f(x, y)\right| \leq M|| f \|_{p}, \quad \forall(x, y) \in \bar{\Omega} .
$$

Tal desigualdade nos diz que $T_{Z} f \in L^{\infty}(\Omega)$ e, mais que isso, $T_{Z}: L^{p}(\Omega) \rightarrow L^{\infty}(\Omega)$, para $p>$ $2+\sigma_{\mathrm{M}}$, é um operador contínuo.

O passo seguinte foi mostrar que $u:=T_{Z} f$, para $f \in L^{1}(\Omega)$, é uma solução da equação $L u=f$ em $\Omega$ quando $L$ é o Hamiltoniano de $Z$. Tal resultado é obtido com o estudo de uma fórmula de representação de soluções do tipo Cauchy-Pompeiu.

Depois de estabelecida a resolubilidade, estudamos a regularidade da função $T_{Z} f$ para $f \in L^{p}(\Omega), p>2+\sigma_{\mathrm{M}}$. Foi mostrado que, neste caso, obtemos regularidade Hölder para $T_{Z} f$. Mais que isso, $T_{Z} f$ satisfaz a seguinte condição de Hölder

$$
\left|T_{Z} f\left(x_{1}, y_{1}\right)-T_{Z} f\left(x_{2}, y_{2}\right)\right| \leq C|| f \|_{p}\left|Z\left(x_{1}, y_{1}\right)-Z\left(x_{2}, y_{2}\right)\right|^{\mu}, \quad \forall\left(x_{1}, y_{1}\right),\left(x_{2}, y_{2}\right) \in \bar{\Omega}
$$

onde $C=C\left(p, \sigma_{1}, \ldots, \sigma_{N}, \Omega\right)>0, \mu=\left(2-2 q-\tau_{\mathrm{M}}\right) / q, q=p /(p-1)$ e $\tau_{\mathrm{M}}=\sigma_{\mathrm{M}} /\left(\sigma_{\mathrm{M}}+1\right)$.

As desigualdades (1.4) e (1.5) nos dizem que o operador $T_{Z}$ tem um bom comportamento como um operador entre os espaços $L^{p}(\Omega), p>2+\sigma_{\mathrm{M}}$, e $C(\bar{\Omega})$. Este bom comportamento nos permitiu aplicar o "método topológico" (ver uma explicação para o significado dessa expressão logo abaixo) no estudo de equações semilineares da forma

$$
L u=F(x, y, u) \text { em } \Omega .
$$

A resolubilidade com regularidade Hölder também foi obtida para a equação acima com $F$ : $\bar{\Omega} \times \mathbb{C} \rightarrow \mathbb{C}$ sendo de duas formas, conforme explicado a seguir. Seja $p>2+\sigma_{\mathrm{M}}$. A primeira forma de $F$ satisfaz

- $F(., \zeta) \in L^{p}(\bar{\Omega})$, para todo $\zeta \in \mathbb{C}$

- $\left|F\left(x, y, \zeta_{1}\right)-F\left(x, y, \zeta_{2}\right)\right| \leq \Psi(x, y)\left|\zeta_{1}-\zeta_{2}\right|^{\alpha}$, para todos $\zeta_{1}, \zeta_{2} \in \mathbb{C}$

onde $\Psi \in L^{p}\left(\bar{\Omega} ; \overline{\mathbb{R}}_{+}\right)$e $0<\alpha<1$. Já a segunda forma de $F$, é a seguinte

$$
F(x, y, \zeta)=g(x, y) Q(x, y, \zeta)+f(x, y)
$$

onde $f, g \in L^{p}(\bar{\Omega})$ e $Q: \bar{\Omega} \times \mathbb{C} \rightarrow \mathbb{C}$ é uma função contínua limitada. 
Tal método topológico se resume a aplicação do Teorema do Ponto Fixo de Shauder ao operador integral contínuo $P: \Lambda_{M_{0}, C_{0}} \rightarrow \Lambda_{M_{0}, C_{0}}$, dado por

$$
P u=T_{Z}(F(\cdot, u))
$$

onde $\Lambda_{M_{0}, C_{0}}$ é um subconjunto não vazio, convexo e compacto de $C(\bar{\Omega})$. Note que grosso modo a continuidade de $P$ segue da continuidade de $T_{Z}$. A partir do ponto fixo e do resultado de resolubilidade da equação $L u=f$ obtemos a solução da equação semilinear.

Para terminar o Capítulo estudamos o Princípio da Similaridade para a equação

$$
L u=a u+b \bar{u} \operatorname{em} \Omega,
$$

o qual nos diz que toda solução contínua da equação pode ser escrita na forma

$$
u=(h \circ Z) e^{S} \text { em } \Omega
$$

onde $h$ é uma função holomorfa em $Z(\Omega)$ e $S \in C^{\mu}(\bar{\Omega})$, para um certo $\mu$, com $0<\mu<1$. A igualdade acima implica que $u$ tem propriedades semelhantes às das soluções do campo (que em geral são escritas da forma $h \circ Z$, com $h$ uma função holomorfa), principalmente quando olhamos para seus zeros, isto é, $u(\mathrm{p})=0$ se, e somente se, $h(Z(\mathrm{p}))=0$.

No Capítulo 6 estudamos problemas de contorno da forma

$$
\left\{\begin{array}{lll}
L u=F(x, y, u) & \text { em } & \Omega, \\
\Re(\bar{\Lambda} u)=\phi & \text { em } & \partial \Omega,
\end{array}\right.
$$

onde $\Omega$ é um aberto simplesmente conexo limitado com bordo suave, $\Lambda \in C^{\alpha}(\partial \Omega),|\Lambda|=1$, $\phi \in C^{\alpha}(\partial \Omega ; \mathbb{R}), 0<\alpha<1$.

A existência de solução do problema acima, como veremos, está relacionada com o sinal do índice da função $\Lambda$ com respeito a $\partial \Omega$ e a orientação da integral primeira do campo.

Para tratar o problema acima, definimos o operador $T_{Z}^{\kappa}$, que é uma modificação do operador $T_{Z}$.

Para $f \in L^{p}(\Omega), p>2+\sigma_{\mathrm{M}}$, e $\kappa \geq 0$ inteiro, operador $T_{Z}^{\kappa}$ é dado por

$$
T_{Z}^{\kappa} f(x, y)=T_{Z} f(x, y)-Z^{2 \kappa}(x, y) B_{Z} f(x, y),
$$

onde

$$
B_{Z} f(x, y) \doteq \frac{1}{2 \pi i} \int_{\Omega} \frac{Z(x, y) \overline{f(\xi, \eta)}}{1-Z(x, y) \overline{Z(\xi, \eta)}} d \xi d \eta .
$$

A função $B_{Z} f$ é limitada por um múltiplo constante da norma $L^{p}$ de $f$. Além disso, $B_{Z} f$ é uma função Hölder contínua em $\bar{\Omega}$ e solução do campo vetorial $L$ em $\Omega$ (i.e., $L\left(B_{Z} f\right)=0 \mathrm{em} \Omega$ ). Podemos concluir assim que $T_{Z}^{K}$ tem boas propriedades como $T_{Z}$, isto é, mantém as propriedades 
de resolubilidade e satisfaz desigualdades do tipo (1.4) e (1.5). Além disso, $B_{Z} f$, como visto acima, quando restrita ao bordo, se comporta como $\overline{T_{Z} f}$. Isto faz com que a função $T_{Z}^{\kappa} f$ satisfaça a seguinte condição de contorno:

$$
\Re\left(\overline{Z^{\kappa}} T_{Z}^{\kappa}\right)=f \operatorname{em} \partial \Omega
$$

Em seguida estudamos o operador de Schwarz $S_{Z}: C(\partial \Omega, \mathbb{R}) \rightarrow C(\bar{\Omega})$ dado por

$$
\left(S_{Z} \varphi\right)(x, y) \doteq \frac{1}{2 \pi i} \int_{\partial \Omega} \varphi(\xi, \eta) \frac{Z(\xi, \eta)+Z(x, y)}{Z(\xi, \eta)-Z(x, y)} \frac{d Z(\xi, \eta)}{Z(\xi, \eta)}
$$

O operador $S_{Z}$ tem a seguinte propriedade: se $\varphi \in C^{\alpha}(\partial \Omega, \mathbb{R}), 0<\alpha<1$, então $S_{Z} \varphi \in C^{\alpha}(\bar{\Omega})$ e satisfaz

$$
\left\{\begin{aligned}
L\left(S_{Z} \varphi\right)=0 & \text { em } \Omega \\
\Re\left(S_{Z} \varphi\right)=\varphi & \text { em } \partial \Omega
\end{aligned}\right.
$$

Utilizando os operadores $T_{Z}^{\kappa}$ e $S_{Z}$ definimos um operador integral da forma

$$
P^{\kappa} u(x, y)=T_{Z}^{\kappa}(F(\cdot, u))(x, y)+Z^{\kappa} S_{Z} \varphi, u \in C(\bar{\Omega}) .
$$

Note que $\Re\left(\overline{Z^{\kappa}} P^{\kappa} u\right)=\varphi$ em $\partial \Omega$.

Existem desigualdades semelhantes às desigualdades (1.4) e (1.5) para o operador $P^{\kappa}$. Assim, podemos usar, de forma análoga ao que foi feito no Capítulo 5, o Teorema do Ponto Fixo de Schauder para obter uma função Hölder contínua $u$ em $\bar{\Omega}$ tal que $P^{\kappa} u=u$. Pensando $L$ como $H_{Z}$, temos que $L u=F(x, y, u)$ em $\Omega$. Além disso, $\Re\left(\bar{Z}^{\kappa} u\right)=\varphi$ em $\partial \Omega$. Com uma mudança de incógnita, que depende do índice de $\Lambda$ com respeito a $\partial \Omega$ e da orientação de $Z$, obtemos uma solução Hölder contínua para o problema (1.8).

O passo posterior foi estudar o seguinte Problema de Riemann-Hilbert:

$$
\left\{\begin{array}{lll}
L u=a u+b \bar{u}+f & \text { em } & \Omega, \\
\Re(\bar{\Lambda} u)=\varphi & \text { em } & \partial \Omega
\end{array}\right.
$$

onde $\Lambda \in C^{\alpha}(\partial \Omega),|\Lambda|=1, \phi \in C^{\alpha}(\partial \Omega ; \mathbb{R}), 0<\alpha<1$, e $a, b, f \in L^{p}(\bar{\Omega}), p>2+\sigma_{\mathrm{M}}$.

$\mathrm{O}$ uso das propriedades do operador integral $T_{Z}^{\kappa}$ com a alternativa de Fredholm nos permitiu estabelecer a resolubilidade do problema em termos do sinal do índice de $\Lambda$. Vale assinalar que a equação tipo Vekua, $L u=a u+b \bar{u}+f$, presente no problema de Riemann-Hilbert (1.12), é diferente do tipo de equação tratada nos problemas anteriores acima. Assim, não foi aplicada a mesma técnica usada no problema precedente. No entanto pela linearidade do termo $a u+b \bar{u}$, na variável $u$, com $u$ no espaço vetorial das funções contínuas em $\bar{\Omega}$ com escalares reais, é possível definir um operador compacto, de $C(\bar{\Omega})$ sobre si mesmo, da forma

$$
G^{\kappa} u=T_{Z}^{\kappa}(a u+b \bar{u})
$$


que se enquadra na teoria de Fredholm. Obtemos uma solução Hölder contínua para o problema usando o Teorema da alternativa de Fredholm para provar a existência de um ponto fixo para uma equação integral envolvendo o operador $G^{\kappa}$.

Para finalizar o capítulo, estudamos o problema (1.12), quando $L$ é hipoelítico, $a$ e $f$ são de classe $C^{\infty}$ e $b$ é a função identicamente nula. Neste caso o problema fica mais simples e pode ser resolvido através da resolubilidade global de $L$ e da teoria clássica do problema de Riemann-Hilbert.

Os Apêndices A e B foram incluídos nesta tese no intuito de tornar o texto mais completo e facilitar a leitura. No Apêndice A listamos alguns teoremas importantes que foram usados no texto e no Apêndice $\mathrm{B}$ falamos de maneira resumida sobre alguns pontos da teoria clássica. $\mathrm{O}$ termo teoria clássica significa a teoria encontrada nos livros (VEKUA, 1962), (BEGEHR, 1994) e (GAKHOV, 1966). 



\section{PRELIMINARES}

\subsection{Espaço de funções}

Seja $\mathbb{Z}_{+}$o conjunto dos números inteiros não negativos $\{0,1,2,3, \ldots\}$.

Em $\mathbb{R}^{2}$ o par ordenado será denotado por $(x, y)$ ou por $(\xi, \eta)$ nesta seção. Seja $\Omega \subset \mathbb{R}^{2}$ um aberto. Denotamos por $C(\Omega)$ o conjunto funções a valores complexos em $\Omega$ que são contínuas, também denotado $C(\Omega, \mathbb{C})$, onde $\mathbb{C}$ denota o conjunto dos números complexos. Assim, seguindo o padrão da notação, denotamos por $C(\Omega, \mathbb{R})$, onde $\mathbb{R}$ denota o conjunto dos números reais, o conjunto das funções definidas em $\Omega$ com valores reais.

O espaço das funções de classe $C^{k}, k \in \mathbb{Z}_{+}$, em $\Omega$, indicado por $C^{k}(\Omega)$, consiste de todas as funções $f$ definidas em $\Omega$ a valores complexos com derivadas parciais contínuas até ordem $k$, isto é, $f \in C^{k}(\Omega)$ se, e somente se,

$$
\frac{\partial^{m+n} f}{\partial x^{m} \partial y^{n}} \in C(\Omega), m, n \in \mathbb{Z}_{+}, \operatorname{com} m+n \leq k
$$

Assumimos a notação $C^{0}(\Omega)=C(\Omega)$. Analogamente, $C^{\infty}(\Omega)$, consiste das funções definidas em $\Omega$ a valores complexos com derivadas parciais contínuas de todas as ordens. Mais explicitamente,

$$
C^{\infty}(\Omega)=\bigcap_{k \in \mathbb{Z}_{+}} C^{k}(\Omega)
$$

O suporte de uma função $f \in C^{0}(\Omega)$, indicado por $\operatorname{supp}(f)$, é o fecho em $\Omega$ do conjunto $\{\mathrm{p} \in \Omega ; f(\mathrm{p}) \neq 0\}$. O suporte de $f$ é o complementar em $\Omega$ do maior aberto onde $f$ é nula.

Indicamos por $C_{c}^{k}(\Omega)$, o subconjunto de $C^{k}(\Omega)$ das funções com suporte compacto e por $C_{c}^{\infty}(\Omega)$ o subconjunto de $C^{\infty}(\Omega)$ das funções de suporte compacto.

$$
C_{c}^{\infty}(\Omega)=\bigcap_{k \in \mathbb{Z}_{+}} C_{c}^{k}(\Omega)
$$


Observação 1. Os conjuntos $C^{k}(\Omega), C^{\infty}(\Omega), C_{c}^{k}(\Omega)$ e $C_{c}^{\infty}(\Omega)$ são espaços vetoriais sobre $\mathbb{K}$, onde $\mathbb{K}$ denota $\mathbb{C}$ ou $\mathbb{R}$, com as operações usuais de funções.

Seja $D=\Omega, \bar{\Omega}$ ou $\Gamma$, onde $\Gamma$ é uma curva em $\mathbb{R}^{2}$.

Dizemos que uma função $f$ satisfaz a condição de Hölder em $D$, com expoente $\alpha$, $0<\alpha \leq 1$, se existe uma constante $K=K(f, \alpha, D)>0$ tal que

$$
\left|f\left(\mathrm{p}_{1}\right)-f\left(\mathrm{p}_{2}\right)\right| \leq K\left|\mathrm{p}_{1}-\mathrm{p}_{2}\right|^{\alpha}, \text { para todos } \mathrm{p}_{1}, \mathrm{p}_{2} \in D
$$

Neste caso, $f$ também é chamada de Hölder contínua quando $0<\alpha<1$ e Lipschitz contínua quando $\alpha=1$. Denotamos por $C^{\alpha}(D)$ o espaço vetorial das funções $f: D \rightarrow \mathbb{C}$ que satisfazem a condição de Hölder em $D$ com expoente $0<\alpha<1$ e denotamos por $\operatorname{Lips}(D)$ o espaço vetorial das funções $f: D \rightarrow \mathbb{C}$ que satisfazem a condição de Hölder em $D$ com expoente $\alpha=1$.

Seja $\tilde{\Omega}$ um aberto de $\mathbb{R}^{2}$ e seja $\Omega$ um aberto de $\mathbb{R}^{2}$, com $\bar{\Omega}$ compacto em $\mathbb{R}^{2}$ tal que $\bar{\Omega} \subset \tilde{\Omega}$. (Em outras palavras, $\Omega$ está compactamente contido em $\tilde{\Omega}$ ). Suponha que $Z: \tilde{\Omega} \rightarrow \mathbb{C}$ é uma função de classe $C^{1}$. Definimos $C_{Z}^{\alpha}(\bar{\Omega})$ como o conjunto das funções $f: \bar{\Omega} \rightarrow \mathbb{C}$ tais que

$$
\left|f\left(\mathrm{p}_{1}\right)-f\left(\mathrm{p}_{2}\right)\right| \leq K\left|Z\left(\mathrm{p}_{1}\right)-Z\left(\mathrm{p}_{2}\right)\right|^{\alpha}
$$

para todos $\mathrm{p}_{1}, \mathrm{p}_{2} \in \bar{\Omega}$, onde $K=K(f, \alpha, \Omega, Z)>0$. Note que $C_{Z}^{\alpha}(\bar{\Omega}) \subset C^{\alpha}(\bar{\Omega})$.

Definimos $C^{k+\alpha}(\Omega), k \in \mathbb{Z}_{+}, 0<\alpha<1$, pelo espaço das funções $f \in C^{k}(\Omega)$ que satisfazem

$$
\frac{\partial^{m+n} f}{\partial x^{m} \partial y^{n}} \in C^{\alpha}(\Omega), m, n \in \mathbb{Z}_{+}, \operatorname{com} m+n=k .
$$

Também é usada a notação $C^{k+\alpha}(\Omega), k \in \mathbb{Z}_{+}, 0 \leq \alpha<1$, que indica apenas que $C^{k+\alpha}(\Omega)=$ $C^{k}(\Omega)$ quando $\alpha=0$. Dizer que uma função $f$ é de classe $C^{k+\alpha}$ em $\Omega$ equivale a dizer que $f \in C^{k+\alpha}(\Omega)$.

Observação 2. Dizer que $\Gamma \subset \mathbb{R}^{2}$ é uma curva de classe $C^{1+\varepsilon}, 0 \leq \varepsilon<1$, equivale a dizer que $\Gamma$ é uma subvariedade mergulhada de dimensão 1, de classe $C^{1+\varepsilon}$, de $\mathbb{R}^{2}$. Em particular, se $\Gamma$ é de classe $C^{1+\varepsilon}$, então $\Gamma$ é uma curva simples. Sobre a teoria de variedades diferenciáveis veja (LIMA, 2007).

Sejam $U$ e $V$ abertos de $\mathbb{R}^{2}$. Um homeomorfismo $F: U \rightarrow V$ é um difeomorfismo de classe $C^{k+\alpha}, k=1,2, \ldots, 0 \leq \alpha<1$, se $F_{1}, F_{2} \in C^{k+\alpha}(U)$ e $\left(F^{-1}\right)_{1},\left(F^{-1}\right)_{2} \in C^{k+\alpha}(V)$, onde $F_{j}$ denota as funções coordenadas de $F$ e $\left(F^{-1}\right){ }_{j}$ denota as funções coordenadas de $F^{-1}$.

Seja $\Omega$ um aberto de $\mathbb{R}^{2}$. Seja $f: \Omega \rightarrow \mathbb{C}$ uma função Lebesgue mensurável que satisfaz

$$
\int_{\Omega}|f(\xi, \eta)|^{p} d \xi d \eta<\infty, 1 \leq p<\infty
$$


O conjunto de tais funções é denotado por $L^{p}(\Omega)$. Como a relação de equivalência $f \approx g$ se e somente, se $f-g=0$ a menos de um conjunto de medida nula, $L^{p}(\Omega)$ é um espaço vetorial com escalares em $\mathbb{K}$ (onde $\mathbb{K}=\mathbb{C}$ ou $\mathbb{R}$ ), com a norma

$$
\|f\|_{p}=\left(\int_{\Omega}|f(\xi, \eta)|^{p} d \xi d \eta\right)^{\frac{1}{p}}
$$

Também denotamos $\|f\|_{p}$ por $\|f\|_{p, \Omega}$. Neste texto, será muito usada a seguinte desigualdade.

Desigualdade de Hölder. Se $f \in L^{p}(\Omega)$ e $g \in L^{q}(\Omega)$, com $1 / p+1 / q=1$, então $f g \in$ $L^{1}(\Omega)$ e vale

$$
\|f g\|_{1} \leq\|f\|_{p}\|g\|_{q}
$$

Definimos por $L^{\infty}(\Omega)$ como o conjunto das funções Lebesgue mensuráveis e limitadas em $\Omega$, com a relação de equivalência $\approx$ descrita acima. Temos que $L^{\infty}(\Omega)$ é um espaço vetorial normado, como $L^{p}(\Omega), 1 \leq p<\infty$, com a norma

$$
\|f\|_{\infty}=\sup \{|f(\xi, \eta)| ;(\xi, \eta) \in \Omega\}
$$

Também denotamos $\|f\|_{\infty}$ por $\|f\|_{\infty, \Omega}$. Para este espaço também vale a desigualdade de Hölder, isto é, se $f \in L^{1}(\Omega)$ e $g \in L^{\infty}(\Omega)$, então $f g \in L^{1}(\Omega)$ e

$$
\|f g\|_{1} \leq\|f\|_{1}\|g\|_{\infty}
$$

O conjunto das funções localmente integráveis em $\Omega$, indicado por $L_{l o c}^{1}(\Omega)$, é o conjunto das funções $f$ Lebesgue mensuráveis em $\Omega$ tais que

$$
\int_{K}|f(\xi, \eta)| d \xi d \eta<\infty
$$

para todo $K \subset \Omega$ compacto.

Ressaltamos que $L^{p}(\Omega) \subset L_{l o c}^{1}(\Omega), 1 \leq p \leq \infty$.

Definição 1. Seja $f: U \rightarrow \mathbb{C}$ uma função contínua, onde $U$ é um aberto de $\mathbb{C}$. Dizemos que $f$ é holomorfa (ou analítica) em $U$, se para cada $z_{0} \in U$ existe o limite

$$
f^{\prime}\left(z_{0}\right)=\lim _{\zeta \rightarrow 0} \frac{f\left(z_{0}+\zeta\right)-f\left(z_{0}\right)}{\zeta}
$$

Se $f$ é holomorfa em $U$ então a função obtida acima, $U \ni z \mapsto f^{\prime}(z) \in \mathbb{C}$ é chamada de derivada de $f$ e é denotada por $f^{\prime}$.

Sejam $U, V$ abertos de $\mathbb{C}$ e $f: U \rightarrow V$ é uma bijeção. Se $f$ é holomorfa em $U$ e $f^{-1}$ é holomorfa em $V$, então $f$ é dita um biholomorfismo de $U$ em $V$. 


\subsection{Distribuições}

A seguir definiremos o conceito de distribuições e faremos alguns comentários. Para um estudo aprofundado veja (HöRMANDER, a).

Um funcional linear de $C_{c}^{\infty}(\Omega)$ sobre $\mathbb{C}$ é uma função $u: C_{c}^{\infty}(\Omega) \rightarrow \mathbb{C}$ tal que

$$
u(a \phi+b \psi)=a u(\phi)+b u(\psi), \text { para } a, b \in \mathbb{C} \text { e } \phi, \psi \in C_{c}^{\infty}(\Omega)
$$

Denotaremos $\langle u, \varphi\rangle$ a ação de um funcional linear $u: C_{c}^{\infty}(\Omega) \rightarrow \mathbb{C}$ em sobre $\varphi \in C_{c}^{\infty}(\Omega)$, isto é, $\langle u, \varphi\rangle=u(\phi)$.

Definição 2. Uma distribuição em $\Omega$ é um funcional linear $u: C_{c}^{\infty}(\Omega) \rightarrow \mathbb{C}$ tal que para cada compacto $K \subset \Omega$, existe uma constante $C$ e um inteiro positivo $m$ tais que

$$
|\langle u, \varphi\rangle| \leq C \sum_{j+\ell \leq m} \sup _{K}\left|\frac{\partial^{j+\ell} \varphi}{\partial x^{j} \partial y^{\ell}}\right|,
$$

para toda $\varphi \in C_{c}^{\infty}(\Omega) \operatorname{com} \operatorname{supp}(\varphi) \subset K$.

Denotamos por $\mathscr{D}^{\prime}(\Omega)$ o conjunto de todas as distribuições em $\Omega$.

$\mathrm{Na}$ definição acima, se for possível determinar o mesmo $m$ para todo compacto $K$, dizemos que $u$ tem ordem menor ou igual a $m$. O conjunto das distribuições em $\Omega$ com ordem menor ou igual a $m \in \mathbb{Z}_{+}$é denotado por $\mathscr{D}_{m}^{\prime}(\Omega)$.

Vamos ver agora alguns exemplos de distribuições

Exemplo 1. Seja $f \in L_{l o c}^{1}(\Omega)$. Então

$$
\left\langle u_{f}, \varphi\right\rangle=\int_{\Omega} f(\xi, \eta) \varphi(\xi, \eta) d \xi d \eta, \varphi \in C_{c}^{\infty}(\Omega),
$$

define uma distribuição em $\Omega$ de ordem zero.

Observação 3. A aplicação $L_{l o c}^{1}(\Omega) \ni f \mapsto u_{f} \in \mathscr{D}^{\prime}(\Omega)$ é linear e injetora. Logo $L_{l o c}^{1}(\Omega)$ pode ser considerado como um subespaço de $\mathscr{D}^{\prime}(\Omega)$. Devido a isso, a distribuição $u_{f}$ será denotada por $f$. Em particular, toda função de classe $C^{k}(\Omega), k=0,1,2, \ldots, \infty$ define uma distribuição em $\Omega$.

Uma distribuição $u \in \mathscr{D}^{\prime}(\Omega)$ é dita $C^{k+\alpha}, k \in \mathbb{Z}_{+}, 0 \leq \alpha<1,\left(C^{\infty}\right)$ no aberto $U \subset \Omega$, se existe uma função $f \in C^{k+\alpha}(U)\left(C^{\infty}(U)\right)$ tal que

$$
\langle u, \varphi\rangle=\int_{U} f(\xi, \eta) \varphi(\xi, \eta) d \xi d \eta, \text { para toda } \varphi \in C_{c}^{\infty}(U) .
$$

Agora, vamos falar um pouco sobre derivação e multiplicação por funções no contexto de distribuições. 
Definição 3. Se $u \in \mathscr{D}^{\prime}(\Omega)$ e $j, \ell \in \mathbb{Z}_{+}$, definimos

$$
\left\langle\frac{\partial^{j+\ell} u}{\partial x^{j} \partial^{\ell}}, \varphi\right\rangle \doteq(-1)^{j+\ell}\left\langle u, \frac{\partial^{j+\ell} \varphi}{\partial x^{j} \partial^{\ell}}\right\rangle, \text { para } \varphi \in C_{c}^{\infty}(\Omega)
$$

e se $f \in C^{\infty}(\Omega)$, definimos

$$
\langle f u, \varphi\rangle \doteq\langle u, f \varphi\rangle, \operatorname{para} \varphi \in C_{c}^{\infty}(\Omega)
$$

Segue de facilmente da definição de distribuição que (2.1) e (2.2) definem distribuições que denotaremos, respectivamente, por $\frac{\partial^{j+\ell} u}{\partial x^{j} \partial^{\ell}}$ e $f u$.

Observação 4. Também temos, mas não de maneira imediata, que (2.2) define uma distribuição $f u \in \mathscr{D}_{m}^{\prime}(\Omega)$ se $f \in C^{m}(\Omega)$ e $u \in \mathscr{D}_{m}^{\prime}(\Omega)$. Veja (HöRMANDER, a), página 55.

Observação 5. As definições acima e espaços de funções estabelecidos para conjuntos em $\mathbb{R}^{2}$ podem ser definidos da mesma forma para conjuntos de $\mathbb{C}$ pela identificação $\mathbb{R}^{2} \ni(x, y) \mapsto$ $x+i y \in \mathbb{C}$. Por exemplo, seja $D$ um aberto de $\mathbb{C}$. Dizemos que uma função $f: D \rightarrow \mathbb{C}$ é de classe $C^{k+\alpha}$, isto é, $f \in C^{k+\alpha}(D), k \in Z^{+}, 0 \leq \alpha<1$, se e somente se, a função $\tilde{f}: \tilde{D} \rightarrow \mathbb{C}$, com $\tilde{D}=\left\{(x, y) \in \mathbb{R}^{2} ; x+i y \in D\right\}$, dada por $\tilde{f}(x, y)=f(x+i y)$, é tal que $\tilde{f} \in C^{k+\alpha}(\tilde{D})$.

\subsection{Campos vetoriais}

Seja $\tilde{\Omega} \subset \mathbb{R}^{2}$ um aberto. Um campo vetorial de classe $C^{k+\alpha}, k=0,1, \ldots, \infty, 0 \leq \alpha<1$, é uma aplicação de $L: C^{1}(\Omega) \rightarrow C(\Omega)$ dada por

$$
L=A(x, y) \frac{\partial}{\partial x}+B(x, y) \frac{\partial}{\partial y}
$$

onde $A, B \in C^{k+\alpha}(\tilde{\Omega})$.

Definição 4. Dizemos que um campo $L$, como em (2.3), é não singular no ponto $\mathrm{p} \in \tilde{\Omega}$ se $|A(\mathrm{p})|+|B(\mathrm{p})|>0$. O campo $L$ é chamdo de não singular em $\Omega$ se $L$ é não singular em cada ponto de $\tilde{\Omega}$.

O vetor conjugado de $L$, como em (2.3), denotado por $\bar{L}$, é o campo

$$
\bar{L}=\bar{A}(x, y) \frac{\partial}{\partial x}+\bar{B}(x, y) \frac{\partial}{\partial y}
$$

onde $\bar{A}$ e $\bar{B}$ são os conjugados das funções $A$ e $B$, respectivamente. O simbolo de $L$ é dado por

$$
L((x, y),(\xi, \eta))=A(x, y) \xi+B(x, y) \eta,
$$

$\operatorname{para}(x, y) \in \tilde{\Omega} \mathrm{e}(\xi, \eta) \in \mathbb{R}^{2}$. 
Definição 5. O campo vetorial $L$ é chamado elítico em $(x, y) \in \tilde{\Omega}$ quando

$$
L((x, y),(\xi, \eta)) \neq 0 \text { para todo }(\xi, \eta) \in \mathbb{R}^{2} \backslash\{(0,0)\} .
$$

O campo vetorial $L$ é chamado de elítico em $\tilde{\Omega}$ se é elítico em todo $(x, y) \in \tilde{\Omega}$.

Observe que se $L$ é elítico, então $L$ é não singular.

Proposição 1. Seja $L=A \partial / \partial x+B \partial / \partial y$ um campo de classe $C^{0}$ em $\tilde{\Omega}$ e seja $p \in \tilde{\Omega}$. As seguintes afirmações são equivalentes:

(a) L é elítico em p;

(b) $L_{p}$ e $\bar{L}_{p}$ são linearmente independentes em p;

(c) $\mathfrak{I}(A \bar{B})(\mathrm{p}) \neq 0$.

Demonstração. Suponha (a). Temos em particular, $A(\mathrm{p}) \neq 0$ e $B(\mathrm{p}) \neq 0$. Assim,

$$
A(\mathrm{p}) \xi+B(\mathrm{p}) \eta=A(\mathrm{p})\left(\xi+\Re\left(\frac{\overline{A(\mathrm{p})} B(\mathrm{p})}{|A(\mathrm{p})|^{2}}\right) \eta+i \mathfrak{I}\left(\frac{\overline{A(\mathrm{p})} B(\mathrm{p})}{|A(\mathrm{p})|^{2}}\right) \eta\right)
$$

onde $(\xi, \eta) \in \mathbb{R}^{2}$. Daí, segue que $\mathfrak{I}\left(\frac{\overline{A(\mathrm{p})} B(\mathrm{p})}{|A(\mathrm{p})|^{2}}\right) \neq 0$, o que equivale a $\mathfrak{I}(A \bar{B})(\mathrm{p}) \neq 0$. Portanto, (a) $\Rightarrow$ (c). Por outro lado, $\mathfrak{I}(A \bar{B})(\mathrm{p}) \neq 0$ implica em particular que $A(\mathrm{p}) \neq 0$. Logo vale (2.4), daí $A(\mathrm{p}) \xi+B(\mathrm{p}) \eta=0$ e $\mathfrak{I}(A \bar{B})(\mathrm{p}) \neq 0$ se, e somente se, $(\xi, \eta)=(0,0)$. Portanto, (c) $\Rightarrow(\mathrm{a})$. Observe que,

$$
2 i \mathfrak{I}(A \bar{B})(\mathrm{p})=\left|\begin{array}{ll}
A(\mathrm{p}) & \bar{A}(\mathrm{p}) \\
B(\mathrm{p}) & \bar{B}(\mathrm{p})
\end{array}\right|
$$

Segue de (2.5) que (c) $\Leftrightarrow$ (b).

Seja $L=A \partial / \partial x+B \partial / \partial y$ um campo de classe $C^{0}$ em $\tilde{\Omega}$. O conjunto

$$
\Sigma(\tilde{\Omega}, L)=\{(x, y) \in \tilde{\Omega} \mid \mathfrak{I}(A \bar{B})(x, y)=0\}
$$

é chamado de conjunto característico de $L$. Quando não há perigo de confusão denotamos $\Sigma(\tilde{\Omega}, L)$ por $\Sigma$. Note que $\Sigma$ é fechado e $L$ restrito a $\tilde{\Omega} \backslash \Sigma$ é elítico.

Exemplo 2. No contexto de funções de variáveis complexas surgem naturalmente os campos elíticos

$$
\frac{\partial}{\partial \bar{z}}=\frac{1}{2}\left(\frac{\partial}{\partial x}+i \frac{\partial}{\partial y}\right) \text { e } \frac{\partial}{\partial z}=\frac{1}{2}\left(\frac{\partial}{\partial x}-i \frac{\partial}{\partial y}\right), z=x+i y,
$$

definidos em $\mathbb{R}^{2}$ (ou em $\mathbb{C}$, pela identificação $x+i y=(x, y)$ ). Eles são chamados, respectivamente, de operador de Cauchy-Riemann e anti-operador de Cauchy-Riemann. 
Observação 6. É conhecido na Análise Complexa o seguinte fato. Seja $h: U \rightarrow \mathbb{C}$ uma função de classe $C^{1}$ no aberto $U \subset \mathbb{R}^{2}$. Se

$$
\frac{\partial h}{\partial \bar{z}}=0 \mathrm{em} U
$$

então $h$ é uma função holomorfa em $U$, tendo em mente a identificação de $\mathbb{R}^{2}$ com $\mathbb{C}$. Isto é, $\tilde{h}(z):=h(x, y)$ definida em no aberto $\tilde{U}=\{z=x+i y ;(x, y) \in U\} \subset \mathbb{C}$ possui derivada complexa (veja a definção 1). Se $h$ é holomorfa, vale $\frac{\partial h}{\partial \bar{z}}(x, y)=\tilde{h}^{\prime}(x+i y)$.

Proposição 2. Seja $L$ um campo em $\tilde{\Omega}$. Se $f \in C^{1}(U)$, onde $U$ é um aberto de $\tilde{\Omega}$, e $h: V \rightarrow \mathbb{C}$ é uma função holomorfa, onde $V$ é um aberto de $\mathbb{C}$ tal que $f(U) \subset V$, então

$$
L(h \circ f)=\left(h^{\prime} \circ f\right) L(f) \text { em } U .
$$

Em particular, quando $f$ é solução de $L$ em $U$, i.e., $L(f)=0$ em $U$, então $h \circ f$ é solução do campo $L$ em $U$, i.e., $L(h \circ f)=0$ em $U$.

Demonstração. A demonstração segue do teorema da regra da cadeia para funções de variáveis reais.

Definição 6. Seja $L$ um campo vetorial de classe $C^{0}$, definido no aberto $\tilde{\Omega}$ de $\mathbb{R}^{2}$. Uma função $Z: U \rightarrow \mathbb{C}$ de classe $C^{1}$, definida no aberto $U \subset \tilde{\Omega}$, é dita uma integral primeira de $L$ em $U$ se $L Z=0$ em $U$ e $d Z \neq 0$ em $U$. Dizemos que $L$ é localmente integrável se para cada $\mathrm{p} \in \tilde{\Omega}$ existir uma integral primeira de $L$ definida em uma vizinhança de $\mathrm{p}$.

Seja $L$ um campo vetorial complexo em $\tilde{\Omega}$. Seja $Z: U \rightarrow \mathbb{C}$ uma integral primeira de $L$ em $U$, onde $U$ é um aberto de $\tilde{\Omega}$, e seja $h: V \rightarrow \mathbb{C}$, onde $V$ é um aberto de $\mathbb{C} \operatorname{com} Z(U) \subset V$, uma função holomorfa com $h^{\prime}(\zeta) \neq 0$ para todo $\zeta \in V$. Segue da Proposição 2 que a composta $h \circ Z$ é uma integral primeira de $L$ em $U$.

Agora, vamos estabelecer a notação para mudança de variáveis em campo vetorial complexo.

Seja $L$ um campo vetorial complexo definido no aberto $\tilde{\Omega} \subset \mathbb{R}^{2}$. Sejam $\Omega_{1}, \Omega_{2}$ abertos de $\mathbb{R}^{2}$ com $\Omega_{1} \subset \tilde{\Omega}$. Denotaremos por $(x, y)$ um elemento genérico de $\Omega_{1}$, e por $(s, t)$ um elemento genérico de $\Omega_{2}$. Seja $F: \Omega_{1} \rightarrow \Omega_{2}$, com $F(x, y)=\left(F_{1}(x, y), F_{2}(x, y)\right)=(s, t)$, um difeomorfismo de classe $C^{1}$. O campo vetorial $L$ nas novas variáveis dadas por $F$, que denotaremos por $L_{F}$, é dado por

$$
L_{F}=\left(L\left(F_{1}\right) \circ F^{-1}\right) \frac{\partial}{\partial s}+\left(L\left(F_{2}\right) \circ F^{-1}\right) \frac{\partial}{\partial t} \text { em } \Omega_{2}
$$

Note que, se $Z: \Omega_{1} \rightarrow \mathbb{C}$ é de classe $C^{1}, \operatorname{com} L Z=0$ em $\Omega_{1}$, temos que a função $Z_{F}: \Omega_{2} \rightarrow \mathbb{C}$ dada por

$$
Z_{F}:=Z \circ F^{-1}
$$

é tal que $L_{F} Z_{F}=0$ em $\Omega_{2}$. 
Observação 7. Seja $f \in C^{1}\left(\Omega_{2}\right)$. O campo $L_{F}$, dado em (2.6), pode ser escrito com

$$
L_{F} f(s, t)=\left(L(f \circ F) \circ F^{-1}\right)(s, t),(s, t) \in \Omega_{2} .
$$

Observação 8. A mudança de variáveis em um campo, descrita acima, também é chamada de mudança de coordenadas.

Observação 9. Seja $L$ um campo de classe $C^{\alpha}, 0<\alpha<1$, em $\Omega$. Dado $\mathrm{p} \in \tilde{\Omega} \backslash \Sigma$ existe uma mudança de variáveis, de classe $C^{1+\alpha}$, numa vizinhança de $\mathrm{p}$ tal que $L$ pode ser escrito como múltiplo, não nulo, do operador de Cauchy-Riemann $\partial / \partial \bar{z}$. Note que $L$ é elítico em p. Em termos da notação acima, existe um difeomorfismo $F: U \rightarrow V$ de classe $C^{1+\alpha}$, onde $U, V$ são abertos com $\mathrm{p} \in U \subset \tilde{\Omega} \backslash \Sigma$, tal que

$$
L_{F}=\lambda_{F} \frac{\partial}{\partial \bar{z}} \text { em } V
$$

onde $\lambda \in C^{\alpha}(V)$, com $|\lambda|>0$ em $V$. O difeomorfismo $F$ é obtido através do estudo da equação de Beltrami relacionada ao campo $L$. Veja (COURANT; HILBERT, 1962), na página 350. Veja também (CAMPANA, 2013).

Proposição 3. Seja $L$ um campo elítico de classe $C^{0}$ em $\Omega$. Suponha que $Z: U \rightarrow \mathbb{C}$ é uma integral primeira, de classe $C^{1}$, de $L$, definida no aberto $U \subset \tilde{\Omega}, \operatorname{com} Z: U \rightarrow Z(U) \subset \mathbb{C}$ um homeomorfismo. Se $u \in C^{1}(U)$ é tal que $L u=0$ em $U$, então existe $h: Z(U) \rightarrow \mathbb{C}$ holomorfa tal que $u=h \circ Z$ em $U$.

Demonstração. Como $L$ é elítico temos que os vetores $\operatorname{grad}(\Re(Z))(\mathrm{p})$ e $\operatorname{grad}(\mathfrak{I}(Z))(\mathrm{p})$ são linearmente independentes para todo $\mathrm{p} \in U$. Então $F: U \rightarrow V$, $\operatorname{com} V=F(U)$, dado por

$$
F(x, y)=(\Re(Z)(x, y), \mathfrak{I}(Z)(x, y))
$$

é um difeomorfismo de classe $C^{1}$. Vamos denotar as coordenadas em $V$ por $(s, t)$. Fazendo a mudança de coordenas em $L$ através de $F$ obtemos

$$
L_{F}=\left(L(\bar{Z}) \circ F^{-1}\right) \frac{1}{2}\left(\frac{\partial}{\partial s}+i \frac{\partial}{\partial t}\right)=: \lambda \frac{\partial}{\partial \bar{w}}
$$

onde $w=s+i t$, e $\lambda \in C(V)$, com $|\lambda|>0$ em $U$. Ou seja, $L_{F}$ é um múltiplo do operador de Cauchy-Riemann em $V$. Além disso, $u_{F}=u \circ F^{-1}$ é de classe $C^{1}$ e satisfaz

$$
\frac{\partial u_{F}}{\partial \bar{w}}=0 \mathrm{em} V
$$

Daí temos que $h:=u \circ Z^{-1}$ é uma função holomorfa em $Z(U)$. Segue o resultado.

Seja $L$ um campo vetorial de classe $C^{\infty}$ em $\tilde{\Omega}$. Por dualidade, seguindo as regras de multiplicação por funções e a regra de diferenciação de distribuições, podemos estender $L$ a uma aplicação de $\mathscr{D}^{\prime}(\tilde{\Omega})$ em si mesmo. Assim, podemos buscar soluções fracas, isto é, soluções distribucionais, de equações envolvendo o campo $L$, por exemplo a equação $L u=f \operatorname{com} f \in$ $C^{\infty}(\tilde{\Omega})$. O próximo teorema ilustra este comentário. 
Teorema 1. Se $u \in \mathscr{D}^{\prime}(\tilde{\Omega})$ é tal que $\frac{\partial u}{\partial \bar{z}}=0$ em $\tilde{\Omega}$, então $u$ é uma função holomorfa em $\tilde{\Omega}$.

Veja demonstração em (HOUNIE, 1979)

Observação 10. Seja $L=A \partial_{x}+B \partial_{y}$ um campo vetorial complexo de classe $C^{0}$ em $\tilde{\Omega}$. Suponha que

$$
\partial_{x} A, \partial_{y} B \in C(\tilde{\Omega}) .
$$

Sejam $f \in C^{1}(\tilde{\Omega})$ e $\phi \in C_{c}^{1}(\tilde{\Omega})$. Utilizando integração por partes e (2.7) temos

$$
\int_{\tilde{\Omega}} L f(x, y) \phi(x, y) d x d y=\int_{\tilde{\Omega}} f(x, y)^{t} L \phi(x, y)
$$

onde

$$
{ }^{t} L=-L-\left(A_{x}+B_{y}\right) .
$$

Ou seja, a relação de dualidade fica bem definida. Tendo em vista essa relação, definimos $L u$ em $\tilde{\Omega}$ e $u \in C(\tilde{\Omega})$, por

$$
\langle L u, \phi\rangle=\left\langle u,{ }^{t} L \phi\right\rangle, \phi \in C_{c}^{1}(\tilde{\Omega}) .
$$

Segue da Observação 4 (página 29) que $L u \in \mathscr{D}_{1}^{\prime}(\tilde{\Omega})$.

Seja $U$ um aberto de $\mathbb{R}^{2}$ e seja $Z: U \rightarrow \mathbb{C}$ uma função de classe $C^{1+k+\alpha}, k=0,1, \ldots, \infty$, $0 \leq \alpha<1$, em $U$. O campo vetorial complexo

$$
H_{Z}:=Z_{y} \frac{\partial}{\partial x}-Z_{x} \frac{\partial}{\partial y} \text { em } U
$$

é um campo vetorial de classe $C^{k+\alpha}$ em $U$. O campo $H_{Z}$ é chamado de Hamiltoniano de $Z$ em $U$.

Proposição 4. Seja $L$ um campo não singular de classe $C^{k+\alpha}, k=0,1, \ldots, \infty, 0 \leq \alpha<1$, em $\tilde{\Omega}$. Se $Z: U \rightarrow \mathbb{C}, U$ aberto de $\tilde{\Omega}$, é uma integral primeira, de classe $C^{1+k+\alpha}$, de $L$, então existe $\lambda \in C^{k+\alpha}(U), \operatorname{com}|\lambda|>0$ em $U$, tal que

$$
L=\lambda H_{Z} \text { em } U
$$

Demonstração. Como $d Z \neq 0$ em $U$ e pela relação $A Z_{x}+B Z_{y}=0$ em $U$ fica bem definida a função $\lambda: U \rightarrow \mathbb{C}$ dada por

$$
\lambda(\mathrm{p})= \begin{cases}\frac{A(\mathrm{p})}{Z_{y}(\mathrm{p})}, & \text { se } Z_{y}(\mathrm{p}) \neq 0 \\ -\frac{B(\mathrm{p})}{Z_{x}(\mathrm{p})}, & \text { se } Z_{x}(\mathrm{p}) \neq 0 .\end{cases}
$$

É fácil verificar que $L=\lambda H_{Z}$ em $U$. 
Agora vamos demonstrar que $\lambda \in C^{k+\alpha}(U)$ e $|\lambda|>0$ em $U$.

Seja $\mathrm{p}_{0} \in U$. Temos que $Z_{x}\left(\mathrm{p}_{0}\right) \neq 0$ ou $Z_{y}\left(\mathrm{p}_{0}\right) \neq 0$. Podemos supor, sem perca de generalidade, que $Z_{y}\left(\mathrm{p}_{0}\right) \neq 0$, pois o argumento usado a seguir também funciona para o caso $Z_{x}\left(\mathrm{p}_{0}\right) \neq 0$. Como $Z_{y}\left(\mathrm{p}_{0}\right) \neq 0$, existe $V \subset U$ aberto tal que $Z_{y}(\mathrm{p}) \neq 0$ para todo $\mathrm{p} \in V$. Então, temos

$$
\lambda=\frac{A}{Z_{y}} \mathrm{em} V
$$

É fácil ver que $\lambda$ é de classe $C^{k+\alpha}$ em $V$. Suponha por contradição que existe $q_{0} \in V$ tal que $\lambda\left(\mathrm{q}_{0}\right)=0$. Então, $A\left(\mathrm{q}_{0}\right)=0$. Como

$$
A\left(\mathrm{q}_{0}\right) Z_{x}\left(\mathrm{q}_{0}\right)+B\left(\mathrm{q}_{0}\right) Z_{y}\left(\mathrm{q}_{0}\right)=0 \text { e } Z_{y}\left(\mathrm{q}_{0}\right) \neq 0
$$

temos $B\left(\mathrm{q}_{0}\right)=0$. Contradição com o fado de $L$ ser não singular. Portanto $\lambda$ não tem zeros em $V$.

Pela arbitrariedade de $p_{0}$ temos que o resultado.

Proposição 5. Sejam $L$ um campo elítico de classe $C^{1}$ em $\tilde{\Omega}$ e $Z: U \rightarrow \mathbb{C}$, com $U$ aberto de $\Omega$, uma integral primeira de $L$, de classe $C^{2}$ em $U$, tal que $Z: U \rightarrow Z(U)$ é um homeomorfismo. Se $u \in C(U)$ é tal que $L u=0$ em $U$ então existe uma função holomorfa $h: Z(U) \rightarrow \mathbb{C}$ tal que $u=h \circ Z$ em $U$.

Demonstração. Para começar a demonstração, vamos verificar que $F: U \rightarrow F(U)$, dada por $F(x, y)=(\Re(Z)(x, y), \mathfrak{I}(Z)(x, y))$, é um difeomorfismo. Como $Z(U)$ é um aberto de $\mathbb{C}$ segue que $F(U)$ é um aberto de $\mathbb{R}^{2}$. Podemos escrever

$$
L=\lambda H_{Z} \text { em } U
$$

$\operatorname{com} \lambda \in C^{1}(U)$ e $|\lambda|>0$ em $U$ (Proposição 4). Note que $L$ é elítico em $U$ se, e somente se, $H_{Z}$ é elítico em $U$. Segue daí que $\mathfrak{I}\left(Z_{y} \overline{Z_{x}}\right)(\mathrm{p}) \neq 0$ para todo $\mathrm{p} \in U$ (Proposição 1). Por outro lado, note que

$$
\left|\mathfrak{I}\left(Z_{y} \overline{Z_{x}}\right)\right|=|\operatorname{det}(D F)|
$$

Segue daí que $\operatorname{det}(D F)(\mathrm{p}) \neq 0$ para todo $\mathrm{p} \in U$ e assim concluímos que $F^{-1}$ é de classe $C^{1}$ pelo Teorema da função inversa. Portanto $F$ é um difeomorfismo.

Agora note que

$$
L u=0 \text { em } U, \text { se, e somente se, } H_{Z} u=0 \text { em } U .
$$

De fato, segue do fato de $\lambda, 1 / \lambda \in C^{1}(U)$ e $L u, H_{Z} u \in \mathscr{D}_{1}^{\prime}(U)$.

Seja dado $\psi \in C_{c}^{\infty}(V)$. Defina $\phi=\psi \circ F$. Temos

$$
\left\langle H_{Z} u, \phi\right\rangle=\left\langle u,-H_{Z} \phi\right\rangle=0 .
$$

Por outro lado,

$$
\left\langle u,-H_{Z} \phi\right\rangle=-\int_{U} u(x, y) H_{Z} \phi(x, y) d x d y
$$




$$
=-\int_{F(U)} \frac{u\left(F^{-1}(\xi, \eta)\right)\left(H_{Z} \phi\right)\left(F^{-1}(\xi, \eta)\right)}{\left|\operatorname{det} D F\left(F^{-1}(\xi, \eta)\right)\right|} d \xi d \eta
$$

Note que

$$
\left(H_{Z} \phi\right) \circ F^{-1}=\left(H_{Z}(\psi \circ F)\right) \circ F^{-1}=\left(H_{Z}(\bar{Z}) \circ F^{-1}\right) \partial_{\bar{w}} \psi=: \lambda \partial_{\bar{w}} \psi
$$

onde $w=s+$ it (veja a Observação 7). Temos assim

$$
\int_{V} \frac{u\left(F^{-1}(\xi, \eta)\right) \lambda(\xi, \eta)\left(\partial_{\bar{w}} \psi\right)(\xi, \eta)}{\left|\operatorname{det} D F\left(F^{-1}(\xi, \eta)\right)\right|} d \xi d \eta=0
$$

lembrando que $F(U)=V$. Note que

$$
H_{Z}(\bar{Z})=2 i \mathfrak{I}\left(Z_{y} \bar{Z}_{x}\right)=2 i \operatorname{det}(D F) .
$$

Daí,

$$
\frac{\lambda(\xi, \eta)}{\left|\operatorname{det}(D F)\left(F^{-1}(\xi, \eta)\right)\right|}=\frac{2 i \operatorname{det}(D F)\left(F^{-1}(\xi, \eta)\right)}{\left|\operatorname{det}(D F)\left(F^{-1}(\xi, \eta)\right)\right|}=2 i c
$$

onde $c=1$ ou -1 . Assim, por (2.10),

$$
\int_{V}\left(u \circ F^{-1}\right)(\xi, \eta)\left(\partial_{\bar{w}} \psi\right)(\xi, \eta) d \xi d \eta=0 .
$$

Assim, pela arbitrariedade de $\psi \in C_{c}^{\infty}(V)$, temos

$$
\partial_{\bar{w}}\left(u \circ F^{-1}\right)=0 \text { em } V .
$$

Segue do Teorema 1 que $h: Z(U) \rightarrow \mathbb{C}$ dada por

$$
h=u \circ Z^{-1}
$$

é holomorfa em $Z(U)$. Concluímos assim o teorema.

Neste texto, vamos considerar a seguinte definição de hipocomplexidade:

Definição 7. Um campo vetorial complexo de classe $C^{k+\varepsilon}, k=0,1,2, \ldots, \infty, 0 \leq \varepsilon<1$, é dito $C^{1+k+\varepsilon}$-hipocomplexo em $\tilde{\Omega}$, se para todo $\mathrm{p} \in \tilde{\Omega}$ existir uma integral primeira de classe $C^{1+k+\varepsilon}$, $Z: U \rightarrow \mathbb{C}$, definida em um conjunto aberto $U$, com $\mathrm{p} \in U$, tal que $Z$ é um homeomorfismo sobre sua imagem, isto é, $Z: U \rightarrow Z(U)$ é um homeomorfismo.

Um campo vetorial $C^{1}$-hipocomplexo é chamado simplesmente de hipocomplexo.

Observação 11. Veja em (TREVES, 1992) e (BERHANU; CORDARO; HOUNIE, 2008) outras definições de hipocomplexidade. 
Um conjunto de funções $Z$, como descritas na Definição 7 acima, é chamado de sistema completo de integrais primeiras que são homeomorfismos de classe $C^{1+k+\varepsilon}$ e é denotado da forma $\left\{U, Z_{U}\right\}_{U \in \mathscr{C}}$.

Segue da escrita local, em termos do operador de Cauchy-Riemann, mencionada na Observação 9, que campos elíticos de classe $C^{\alpha}, 0<\alpha<1$, são $C^{1+\alpha}$-hipocomplexos.

Proposição 6. Seja $L$ um campo vetorial $C^{1}$-hipocomplexo definido em um aberto $\tilde{\Omega} \subset \mathbb{R}^{2}$. Seja $Z: U \rightarrow \mathbb{C}$, com $U \subset \tilde{\Omega}$, uma integral primeira de $L$, de classe $C^{1}$, que é um homeomorfismo sobre sua imagem. Suponha que o conjunto característico $\Sigma$ de $L$ é uma subvariedade mergulhada de dimensão 1 de $\tilde{\Omega}$ de classe $C^{1}$. Se $u \in C^{1}(U)$ é tal que $L u=0$ em $U$ então existe $h: Z(U) \rightarrow \mathbb{C}$ holomorfa tal que $u=h \circ Z$ em $U$.

Demonstração. Defina $h: Z(U) \rightarrow \mathbb{C}$ por

$$
h=u \circ Z^{-1} \text {. }
$$

Temos que $h$ é uma função contínua em $Z(U)$. Seja $W=Z(U \backslash(\Sigma \cap U))$. Segue da Proposição 3 (página 32) que $\left.h\right|_{W}$ é holomorfa. Vamos verificar que $h$ é holomorfa numa vizinhança de cada ponto de $\zeta \in Z(\Sigma \cap U)$. Seja dado $\zeta_{0} \in Z(\Sigma \cap U)$. Seja $p_{0}=Z^{-1}\left(\zeta_{0}\right)$. Como $\Sigma$ é uma união disjunta de curvas de classe $C^{1}$ existe $\delta>0$ tal que $\overline{B\left(\mathrm{p}_{0}, \delta\right)} \subset U$ e $\overline{B\left(\mathrm{p}_{0}, \delta\right)} \cap \Sigma$ é um arco de curva que divide $B\left(\mathrm{p}_{0}, \delta\right)$ em dois abertos conexos. Além disso, como $Z$ é de classe $C^{1}$ em $U$ e $\overline{B\left(\mathrm{p}_{0}, \delta\right)} \subset U$ é compacto, segue que $Z$ é Lipschitz em $\overline{B\left(\mathrm{p}_{0}, \delta\right)}$. Segue daí que $Z\left(\overline{B\left(\mathrm{p}_{0}, \delta\right)} \cap \Sigma\right)$ é um arco de curva contínua retificável. Sejam $D=Z\left(B\left(\mathrm{p}_{0}, \delta\right)\right)$ e $\Gamma=Z\left(B\left(\mathrm{p}_{0}, \delta\right) \cap \Sigma\right)$. Como $Z$ é um homeomorfismo, temos que $\Gamma$ divide $D$ em duas componentes conexas $D_{1}$ e $D_{2}$. Temos os seguintes fatos: $h$ é contínua em $D$ e a restrição $h_{j}: D_{j} \rightarrow \mathbb{C}, j=1,2$ dada por $h_{j}=h$ em $D_{j}$, é holomorfa (como vimos acima). Por um resultado de análise complexa (ver Teorema 23, página 132) $h_{1}$ e $h_{2}$ formam uma continuação holomorfa uma da outra, ou seja, $h$ é holomorfa em $D$. Como $D$ é uma vizinhança de $\zeta_{0}$, e $\zeta_{0}$ é arbitrário, segue que $h$ é holomorfa numa vizinhança de $Z(\Sigma \cap U)$ e daí concluímos que $h$ é holomorfa em $Z(U)$.

A próxima proposição exibe mais uma boa propriedade de campos hipocomplexos: a existência de integral primeira global que é um homeomorfismo sobre sua imagem, que em geral denotamos por $Z$. Essa propriedade é fundamental neste texto. Muitos problemas e técnicas que envolvem o operador de Cauchy-Riemann $\partial / \partial \bar{z}$ podem ser generalizadas para $L$ através de $Z$. Um exemplo é a generalização do operador integral clássico $T f$ (definido em (B.2), página 136) para o operador integral $T_{Z} f$ (definido em (5.5), página 72). Na teoria clássica $T f$ é usado no estudo da equação $\partial u / \partial \bar{z}=f$, enquanto que aqui usaremos, sua generalização, $T_{Z} f$ para o estudo da equação $L u=f$ (capítulo 5, página 71).

A demonstração da seguinte proposição é inspirada em (BERGAMASCO; CORDARO; HOUNIE, 1988). 
Proposição 7. Seja $L$ um campo vetorial definido no aberto conexo $\tilde{\Omega}$ de $\mathbb{R}^{2}$. Suponha que $L$ é um campo $C^{1+\varepsilon}$-hipocomplexo, $0 \leq \varepsilon<1$, e que seu conjunto característico $\Sigma$ é uma subvariedade mergulhada de $\tilde{\Omega}$ de dimensão 1 e classe $C^{1}$. Então $L$ possui uma integral primeira global em $\tilde{\Omega}$ que é um homeomorfismo sobre sua imagem. Mais precisamente, existe uma função

$$
Z: \tilde{\Omega} \rightarrow Z(\tilde{\Omega}) \subset \mathbb{C}
$$

de classe $C^{1+\varepsilon}$, tal que $Z$ é um homeomorfismo, $L Z=0$ e $d Z \neq 0$ em $\tilde{\Omega}$.

Demonstração. Como $L$ é $C^{1+\varepsilon}$-hipocomplexo existe uma cobertura aberta $\left\{U_{v}\right\}_{v \in I}$ de $\tilde{\Omega}$ e para cada $U_{v}$ existe uma aplicação $Z_{v}: U_{v} \rightarrow \mathbb{C}$, de classe $C^{1+\varepsilon}$, tal que $L Z_{v}=0, d Z_{v} \neq 0$ e $Z_{v}: U_{v} \rightarrow Z(U) \subset \mathbb{C}$ é um homeomorfismo. Pela Proposição 6 a família $\left\{U_{v}, Z_{v}\right\}_{v \in I}$ é um atlas de $\tilde{\Omega}$, isto é, $Z_{v}: U_{v} \rightarrow Z_{v}\left(U_{v}\right)$ é um homeomorfismo do aberto $U_{v}$ de $\Omega$ sobre o aberto $Z_{v}\left(U_{v}\right)$ em $\mathbb{C},\left\{U_{v}\right\}_{v \in I}$ é uma cobertura de $\Omega$ e se $U_{v} \cap U_{\mu} \neq 0, v, \mu \in I$, então

$$
Z_{v} \circ Z_{\mu}^{-1}: Z_{\mu}\left(U_{v} \cap U_{\mu}\right) \longrightarrow Z_{v}\left(U_{v} \cap U_{\mu}\right)
$$

é uma função holomorfa. Como $\tilde{\Omega}$ é conexo, a família $\left\{U_{v}, Z_{v}\right\}_{v \in I}$ define sobre $\tilde{\Omega}$ uma estrutura de superfície de Riemann. Pelo Teorema de uniformização de Riemann (Teorema 22, página 131) existe uma aplicação holomorfa (no sentido da estrutura diferencial de superfície de Riemann de $\tilde{\Omega})$

$$
Z: \tilde{\Omega} \rightarrow Z(\tilde{\Omega}) \subset \mathbb{C}
$$

que é um homeomorfismo sobre sua imagem. Dizer que $Z$ é uma função holomorfa de $\Omega$ para $\mathbb{C}$, no sentido da estrutura diferencial de superfície de Riemann, quer dizer que

$$
h_{v}:=Z \circ Z_{v}^{-1}: Z_{v}\left(U_{v}\right) \longrightarrow \mathbb{C} \text { é holomorfa, no sentido clássico, para todo } v \in I \text {; }
$$

Assim, $\left.Z\right|_{U_{v}}=h_{v} \circ Z_{v}: U_{v} \longrightarrow \mathbb{C}$ para todo $v \in I$. Segue que $Z: \tilde{\Omega} \rightarrow \mathbb{C}$ é de classe $C^{1+\varepsilon}$. Em geral, $Z$ tem a mesma regularidade que a regularidade da família de integrais primeiras usadas como atlas acima. Além disso, $L\left(\left.Z\right|_{U_{v}}\right)=L\left(h_{v} \circ Z_{v}\right)=0$ em $U_{v}$, pois $h_{v}$ é holomorfa e $L\left(Z_{v}\right)=0$ em $U_{v}$. Segue daí que $L Z=0$ em $\tilde{\Omega}$. Para todo $v \in I, d\left(\left.Z\right|_{U_{v}}\right)=h_{v}^{\prime}\left(Z_{v}\right) d Z_{v} \neq 0$ em $U_{v}$, pois $h_{v}: Z_{v}\left(U_{v}\right) \rightarrow Z\left(U_{v}\right)$ é um biholomorfismo e assim $h_{v}^{\prime} \neq 0$ em $Z_{v}\left(U_{v}\right)$ e $d Z_{v} \neq 0$ em $U_{v}$. Segue que $d Z \neq 0$ em $\tilde{\Omega}$.

\subsubsection{Campos vetoriais de tipo finito e infinito}

Seja $L$ um campo vetorial complexo de classe $C^{\infty}$ definido no aberto $\tilde{\Omega}$ de $\mathbb{R}^{2}$. Seja $\Sigma$ seu conjunto característico em $\tilde{\Omega}$.

Um ponto $\mathrm{p}_{0} \in \Sigma$ é dito de tipo finito se existe um campo vetorial $Y$ na álgebra de Lie gerada por $L$ e $\bar{L}$ tal que $L$ e $Y$ são linearmente independentes em $\mathrm{p}_{0}$. Isto significa que existe um colchete de Lie da forma

$$
Y=\left[Y_{1},\left[Y_{2},\left[\ldots\left[Y_{k-1}, Y_{k}\right]\right]\right]\right]
$$


onde $Y_{j}=L$ ou $\bar{L}$, tal que $L$ e $Y$ são linearmente independentes em $\mathrm{p}_{0}$. O tipo de $L$ no ponto $\mathrm{p}_{0}$ é o menor $k$, que aparece na expressão de $Y$ acima, de modo que $L$ e $Y$ sejam linearmente independentes em $\mathrm{p}_{0}$. Se o campo $Y$ não existe, então $\mathrm{p}_{0}$ é dito de tipo infinito. O subconjunto de $\Sigma$ de pontos de tipo finito é denotado por $\Sigma^{0}$ e o subconjunto de pontos de tipo infinito é denotado por $\Sigma^{\infty}$. Temos assim

$$
\Sigma=\Sigma^{0} \cup \Sigma^{\infty} \text { e } \Sigma^{0} \cap \Sigma^{\infty}=\emptyset
$$

Dizemos que $L$ é de tipo finito se $\Sigma=\Sigma^{0}$ e dizemos que $L$ é de tipo infinito se $\Sigma=\Sigma^{\infty}$.

Exemplo 3. Considere os campos

$$
M=\frac{\partial}{\partial y}-i y \frac{\partial}{\partial x} \text { e } N=\frac{\partial}{\partial y}-i x \frac{\partial}{\partial x}
$$

definidos em $\mathbb{R}^{2}$. O campo $M$ é de tipo finito e o campo $N$ é de tipo infinito.

Exemplo 4. Seja $k>0$ um inteiro. O campo

$$
M_{k}=\frac{\partial}{\partial y}-i y^{k} \frac{\partial}{\partial x}
$$

definido em $\mathbb{R}^{2}$ é de tipo finito $k$ em todos os pontos de seu conjunto característico.

\subsection{0 índice}

Seja $\mathrm{p}_{0} \in \mathbb{C}$ e $\alpha:[0,1] \rightarrow \mathbb{C}$ um caminho fechado com $\alpha(t) \neq \mathrm{p}_{0}$, para todo $t \in[0,1]$.

Existe uma função contínua $\tilde{\alpha}:[0,1] \rightarrow \mathbb{R}$ tal que

$$
\alpha(t)=\mathrm{p}_{0}+\rho(t) e^{i \tilde{\alpha}(t)} \text { para todo } t \in[0,1]
$$

onde $\rho(t)=\left|\alpha(t)-\mathrm{p}_{0}\right|$. (Veja (LIMA, 2006)). A função $\tilde{\alpha}$ é chamada de função-ângulo. Qualquer outra função contínua que definida em $[0,1]$ sobre $\mathbb{R}$ que satisfaz (2.11) difere de $\tilde{\alpha}$ por $2 k \pi, \operatorname{com} k \in \mathbb{Z}$. Daí temos que $\mathrm{n}\left(\alpha ; \mathrm{p}_{0}\right)$ definido por

$$
\mathrm{n}\left(\alpha ; \mathrm{p}_{0}\right)=\frac{\tilde{\alpha}(1)-\tilde{\alpha}(0)}{2 \pi}
$$

é um número inteiro que não depende de $\tilde{\alpha}$. O número $\mathrm{n}\left(\alpha ; \mathrm{p}_{0}\right)$ é o número de voltas que $e^{i \tilde{\alpha}}(t)$ dá em $\mathbb{S}^{1}$ no sentido positivo (contrário aos ponteiros do relógio) menos o número de voltas no sentido negativo quando $t$ varia de 0 a 1 . Veja (LIMA, 2006). Veja também (AHLFORS; SARIO, 1960).

Observação 12. Se $a:[0,1] \rightarrow \mathbb{R}^{2}$ é um caminho, definimos $\mathrm{n}\left(a ; \mathrm{p}_{0}\right)$ por $\mathrm{n}\left(\alpha ; \mathrm{p}_{0}\right)$, onde $\alpha$ : $[0,1] \rightarrow \mathbb{C}$ é tal que $a=(\mathfrak{R}(\alpha), \mathfrak{I}(\alpha))$. 
Seja $F: \tilde{\Omega}_{1} \rightarrow \tilde{\Omega}_{2}$ uma aplicação contínua, com $\tilde{\Omega}_{1}, \tilde{\Omega}_{2} \subset \mathbb{R}^{2}$ abertos simplesmente conexos. Seja $\mathrm{p}_{0} \in \tilde{\Omega}$. A aplicação $F$ define um homeomorfismo entre os grupos fundamentais de $\tilde{\Omega}_{1} \backslash\left\{\mathrm{p}_{0}\right\}$ e $\tilde{\Omega}_{2} \backslash\left\{F\left(\mathrm{p}_{0}\right)\right\}$ (que ambos, são isomorfos a $\mathbb{Z}$ ). Mais especificamente,

$$
\mathscr{F}: \pi_{1}\left(\tilde{\Omega}_{1} \backslash\left\{\mathrm{p}_{0}\right\}\right) \rightarrow \pi_{1}\left(\tilde{\Omega}_{2} \backslash\left\{F\left(\mathrm{p}_{0}\right)\right\}\right),
$$

dado por $\mathscr{F}([\alpha])=[F \circ \alpha]$, onde $[\cdot]$ denota a classe de homotopia do caminho. Como $\tilde{\Omega}_{1} \backslash\left\{\mathrm{p}_{0}\right\}$ e $\tilde{\Omega}_{2} \backslash\left\{F\left(\mathrm{p}_{0}\right)\right\}$ são isomorfos a $\mathbb{Z}$, existem $\delta_{F}$ inteiro, $[\alpha]$ um gerador de $\pi\left(\tilde{\Omega}_{1} \backslash\left\{\mathrm{p}_{0}\right\}\right)$ e $[\beta]$ um gerador de $\pi\left(\tilde{\Omega}_{2} \backslash\left\{F\left(\mathrm{p}_{0}\right)\right\}\right)$ tais que

$$
\mathscr{F}\left([\alpha]^{m}\right)=[\beta]^{m \delta_{F}}, \text { para todo } m \in \mathbb{Z} .
$$

Quando $F$ é um homeomorfismo, temos que $\mathscr{F}$ é um isomorfismo e vale

$$
\mathrm{n}\left(F \circ \alpha ; F\left(\mathrm{p}_{0}\right)\right)=\delta_{F} \mathrm{n}\left(\alpha ; \mathrm{p}_{0}\right)
$$

para todo caminho fechado $\alpha$ em $\tilde{\Omega}_{1} \backslash\left\{\mathrm{p}_{0}\right\}$, onde $\delta_{F}=1$ ou $\delta_{F}=-1$. Observe que $\delta_{F}=\delta_{F^{-1}}$. Outro ponto a se observar é que $\delta_{F}$ não depende do ponto $\mathrm{p}_{0}$. Veja (AHLFORS; SARIO, 1960).

Observação 13. Seja $F: \tilde{\Omega}_{1} \rightarrow \tilde{\Omega}_{2}$, com $\tilde{\Omega}_{1}, \tilde{\Omega}_{2} \subset \mathbb{R}^{2}$ abertos simplesmente conexos, um homeomorfismo. Dizemos que $F$ preserva orientação quando $\delta_{F}=1$, e dizemos que $F$ inverte orientação quando $\delta_{F}=-1$. Este conceito de orientação concorda com o conceito de orientação de aplicações suaves dado pelo sinal do determinante da aplicação no seguinte sentido. Suponha $F$ de classe $C^{1}$ e seja $\mathrm{p}_{0} \in \tilde{\Omega}$ tal que $\operatorname{det}(D F)\left(\mathrm{p}_{0}\right) \neq 0$. Se $\operatorname{det}(D F)\left(\mathrm{p}_{0}\right)>0$, então $F$ preserva orientação e se $\operatorname{det}(D F)\left(\mathrm{p}_{0}\right)<0$ então $F$ inverte orientação.

Seja $\Gamma \subset \mathbb{R}^{2}$ uma curva fechada simples de classe $C^{1}$. Seja $G: \Gamma \rightarrow \mathbb{C}$ uma função contínua tal que $|G(\mathrm{p})|=1$ para todo $\mathrm{p} \in \Gamma$ (isto é, $G \in C\left(\Gamma, \mathbb{S}^{1}\right)$ ).

Definição 8. O índice de $G$ com respeito a $\Gamma$ é definido pelo número inteiro

$$
\operatorname{ind}_{\Gamma}(G)=\frac{1}{2 \pi} \int_{\Gamma} d \arg (G)
$$

Observação 14. A integral acima é entendida no sentido da integral de Stieltjes. Veja (GAKHOV, 1966) e (BEGEHR, 1994).

O índice de $G$ com respeito a $\Gamma$, $\operatorname{ind}_{\Gamma}(G)$, pode ser expresso pela seguinte igualdade

$$
\operatorname{ind}_{\Gamma}(G)=\mathrm{n}(G \circ \gamma ; 0)
$$

onde $\gamma:[0,1] \rightarrow \mathbb{R}^{2}$ é uma parametrização, orientada positivamente (sentido anti-horário), de $\Gamma$. Note que $G \circ \gamma$ denota o caminho $G(\gamma(t)), t \in[0,1]$. Veja (GAKHOV, 1966).

Quando não houver perigo de confusão, denotaremos o índice ind $\Gamma(G)$ simplesmente por ind $(G)$. 
Observação 15. $-\operatorname{ind}(G)=\mathrm{n}\left(G \circ \gamma^{-1} ; 0\right)$, onde $\gamma^{-1}(t)=\gamma(t-1), t \in[0,1]$.

Se $G, G_{1}$ e $G_{2} \in C\left(\Gamma, \mathbb{S}^{1}\right)$, então

$$
\operatorname{ind}\left(G_{1} G_{2}\right)=\operatorname{ind}\left(G_{1}\right)+\operatorname{ind}\left(G_{2}\right) \operatorname{eind}(1 / G)=-\operatorname{ind}(G)=\operatorname{ind}(\bar{G}) .
$$

Observação 16. Sejam $G, \Gamma$ e $\gamma$ como acima. Suponha que $\operatorname{ind}_{\Gamma}(G)=0$. Temos que a função $g=G \circ \gamma:[0,1] \rightarrow \mathbb{S}^{1} \subset \mathbb{C}$ é um caminho fechado. Como vimos no início da seção, existe uma função contínua $\tilde{g}:[0,1] \rightarrow \mathbb{R}$ que satisfaz

$$
g(t)=e^{i \tilde{g}(t)}, \text { para todo } t \in[0,1] .
$$

Como

$$
\operatorname{ind}_{\Gamma}(G)=\frac{\tilde{g}(1)-\tilde{g}(0)}{2 \pi}
$$

e $\operatorname{ind}_{\Gamma}(G)=0$, temos que $\tilde{g}(1)=\tilde{g}(0)$. Segue daí que a função $\vartheta: \Gamma \rightarrow \mathbb{R}$ dada por



onde $\mathrm{p}^{*}=\gamma(0)$ (lembrando que $\gamma(0)=\gamma(1)$ ), é contínua. Além disso, a função $\vartheta$ satisfaz

$$
G(\mathrm{p})=e^{i \vartheta(\mathrm{p})}, \text { para todo } \mathrm{p} \in \Gamma .
$$

Sejam $\tilde{\Omega}$ um aberto simplesmente conexo de $\mathbb{R}^{2}, \Gamma$ uma curva fechada simples de classe $C^{1} \mathrm{em} \tilde{\Omega} \mathrm{e}$

$$
L=A(x, y) \frac{\partial}{\partial x}+B(x, y) \frac{\partial}{\partial y},
$$

$\operatorname{com} A, B \in C(\tilde{\Omega})$, um campo não singular $C^{1}$-hipocomplexo em $\Omega$ com conjunto característico sendo uma subvariedade mergulhada de $\tilde{\Omega}$ de dimensão 1 e classe $C^{1}$.

Pela Proposição 7 (página 37) existe uma integral primeira global $Z: \Omega ् \Omega \rightarrow \mathbb{C}$ de $L$, de classe $C^{1}$, tal que $Z: \tilde{\Omega} \rightarrow Z(\tilde{\Omega})$ é um homeomorfismo. Temos que $\tilde{\Omega} \ni \mathrm{p} \mapsto \operatorname{det}(D Z)(\mathrm{p})$ não muda de sinal em $\tilde{\Omega}$. (Essa afirmação segue de um argumento relacionado a orientação e também pode ser provada diretamente. Veja o Lema 2 no fim da seção). Além disso, pela Proposição 4 (página 33) podemos escrever $L$, dado em (2.13), como

$$
L=\lambda H_{Z}=\lambda\left\{\partial_{y} Z \frac{\partial}{\partial x}-\partial_{x} Z \frac{\partial}{\partial y}\right\} \text { em } \tilde{\Omega}
$$

onde $\lambda \in C(\tilde{\Omega})$ e $\lambda(\mathrm{p}) \neq 0$ para todo $\mathrm{p} \in \tilde{\Omega}$. Daí,

$$
A=\lambda \partial_{y} Z \text { e } B=-\lambda \partial_{x} Z
$$


Como

$$
\operatorname{det}(D Z)=\left|\begin{array}{ll}
\partial_{x} \Re(Z) & \partial_{y} \mathfrak{R}(Z) \\
\partial_{x} \mathfrak{I}(Z) & \partial_{y} \mathfrak{I}(Z)
\end{array}\right|=\mathfrak{I}\left(\overline{\partial_{x} Z} \partial_{y} Z\right) \text { em } \tilde{\Omega}
$$

temos

$$
\operatorname{det}(D Z)=\mathfrak{I}\left(\overline{\partial_{x} Z} \partial_{y} Z\right)=-\mathfrak{I}\left(\overline{\left(\frac{B}{\lambda}\right)} \frac{A}{\lambda}\right)=-\frac{1}{|\lambda|^{2}} \mathfrak{I}(A \bar{B}) \text { em } \tilde{\Omega} .
$$

Em particular, $\tilde{\Omega} \ni \mathrm{p} \mapsto \mathfrak{I}(A \bar{B})(\mathrm{p})$ não muda de sinal em $\tilde{\Omega}$. Segue de (2.14) que $Z$ preserva orientação quando o sinal de $\mathfrak{I}(A \bar{B})\left(\mathrm{p}_{0}\right), \mathrm{p}_{0} \in \tilde{\Omega} \backslash \Sigma$, é negativo e inverte orientação quando $\mathfrak{I}(A \bar{B})\left(\mathrm{p}_{0}\right)$ é positivo.

Definição 9. Definimos por

$$
\operatorname{Ind}_{L, \Gamma}(G)=-\operatorname{sgn}\left(\mathfrak{I}(A \bar{B})\left(\mathrm{p}_{0}\right)\right) \operatorname{ind}_{\Gamma}(G)
$$

onde $\mathrm{p}_{0}$ é um ponto qualquer de $\tilde{\Omega} \backslash \Sigma$ e sgn denota a função sinal, isto é, sgn : $\mathbb{R} \backslash\{0\} \rightarrow\{-1,1\}$, $\operatorname{sgn}(x)=1$ se $x>0$ e $\operatorname{sgn}(x)=-1$ se $x<0$.

Segue da definição acima que $\operatorname{Ind}_{L, \Gamma}(G)$ possui as mesmas propriedades do índice clássico listadas em (2.12), isto é,

$$
\operatorname{Ind}_{L, \Gamma}\left(G_{1} G_{2}\right)=\operatorname{Ind}_{L, \Gamma}\left(G_{1}\right)+\operatorname{Ind}_{L, \Gamma}\left(G_{2}\right) \text { e } \operatorname{Ind}_{L, \Gamma}(1 / G)=-\operatorname{Ind}_{L, \Gamma}(G)=\operatorname{Ind}_{L, \Gamma}(\bar{G})
$$

Seja $j: \mathbb{C} \rightarrow \mathbb{R}^{2}$ a aplicação de identificação, i.e., $j(z)=(\Re(z), \mathfrak{I}(z))$. Definimos $\tilde{Z}=$ $j \circ Z$. Então $\tilde{Z}(\tilde{\Omega})$ é um aberto de $\mathbb{R}^{2}$ e $\tilde{Z}: \tilde{\Omega} \rightarrow \tilde{Z}(\tilde{\Omega})$ é um homeomorfismo.

Seja $\Gamma$ uma curva fechada simples de classe $C^{1}$ em $\tilde{\Omega}$ tal que $\tilde{Z}(\Gamma)$ é uma curva fechada simples de classe $C^{1}$ em $\tilde{Z}(\tilde{\Omega})$.

Seja $G \in C(\Gamma)$, com $|G(\mathrm{p})|=1$ para todo $\mathrm{p} \in \Gamma$. Temos que $\tilde{G}:=G \circ \tilde{Z}^{-1} \in C(\tilde{Z}(\Gamma))$ e $|\tilde{G}(\mathrm{p})|=1$ para todo $\mathrm{p} \in \Gamma$.

Lema 1. Vale a seguinte igualdade:

$$
\operatorname{ind}_{\tilde{Z}(\Gamma)}(\tilde{G})=\delta_{\tilde{Z}} \operatorname{ind}_{\Gamma}(G)
$$

Demonstração. Suponha que $\gamma:[0,1] \rightarrow \mathbb{R}^{2}$ é uma parametrização orientada positivamente de $\tilde{Z}(\Gamma)$. Seja $\delta=\delta_{\tilde{Z}}$. Assim,

$$
\left(\tilde{Z}^{-1} \circ \gamma\right)^{\delta}:[0,1] \rightarrow \mathbb{R}^{2}
$$

é uma parametrização orientada positivamente de $\Gamma$. Então podemos escrever

$$
\operatorname{ind}(G)=\mathrm{n}\left(G \circ\left(\left(\tilde{Z}^{-1} \circ \gamma\right)^{\delta}\right) ; 0\right) .
$$

Assim,

$$
\delta \operatorname{ind}(G)=\delta \mathrm{n}\left(G \circ\left(\tilde{Z}^{-1} \circ \gamma\right)^{\delta} ; 0\right)=\mathrm{n}\left(G \circ\left(\left(\tilde{Z}^{-1} \circ \gamma\right)^{\delta^{2}}\right) ; 0\right)
$$




$$
=\mathrm{n}\left(\left(G \circ \tilde{Z}^{-1}\right) \circ \gamma ; 0\right)=\mathrm{n}(\tilde{G} \circ \gamma ; 0)=\operatorname{ind}(\tilde{G}) .
$$

Proposição 8. Seja $G \in C(\Gamma)$, com $|G(\mathrm{p})|=1$ para todo $\mathrm{p} \in \Gamma$. Vale a igualdade

$$
\operatorname{Ind}_{L, \Gamma}(G)=\operatorname{ind}_{\tilde{Z}(\Gamma)}(\tilde{G}) .
$$

Demonstração. Sabemos que $\delta_{\tilde{Z}}$ é dado por $\operatorname{sgn}\left(\operatorname{det}(D \tilde{Z})\left(\mathrm{p}_{0}\right)\right)$ para um $\mathrm{p}_{0} \in \tilde{\Omega}$ tal que $\operatorname{det}(D \tilde{Z})\left(\mathrm{p}_{0}\right) \neq$ 0 ou equivalentemente para $\mathrm{p}_{0} \in \tilde{\Omega} \backslash \Sigma$, onde $\operatorname{sgn}(x)=1$ se $x>0$ e $\operatorname{sgn}(x)=-1$ se $x<0$. Por outro lado,

$$
\delta_{\tilde{Z}}=\operatorname{sgn}\left(\operatorname{det}\left(D Z\left(\mathrm{p}_{0}\right)\right)\right)=\operatorname{sgn}\left(\operatorname{det}\left(D \tilde{Z}\left(\mathrm{p}_{0}\right)\right)\right)=-\operatorname{sgn}\left(\mathfrak{I}(A \bar{B})\left(\mathrm{p}_{0}\right)\right) .
$$

Pelo Lema 1 temos o resultado.

Observação 17. Seja $h: Z(\tilde{\Omega}) \rightarrow \mathbb{C}$ uma função de classe $C^{1}, \operatorname{com} h: Z(\tilde{\Omega}) \rightarrow W, W=h(Z(\tilde{\Omega}))$, um homeomorfismo e $\operatorname{det}(D h)(\zeta)>0$ para todo $\zeta \in Z(\tilde{\Omega})$. Considere $\mathscr{Z}: \tilde{\Omega} \rightarrow \mathbb{C}$ dada por

$$
\mathscr{Z}=h \circ Z \text {. }
$$

A proposição anterior vale quando trocamos $Z$ por $\mathscr{Z}$. Note que $\mathscr{Z}$ tem a mesma orientação que $Z$.

O lema a seguir está relacionado com as condições $(P)$ e $(Q)$ descritas em (TREVES, 1971).

Lema 2. Seja $L$ um campo $C^{1}$-hipocomplexo definido no aberto conexo $\tilde{\Omega}$. Seja $Z: \tilde{\Omega} \rightarrow \mathbb{C}$ uma integral primeira global de $L$, de classe $C^{1}$, que é um homeomorfismo sobre sua imagem. Então a função

$$
\tilde{\Omega} \ni \mathrm{p} \rightarrow \operatorname{det}(D Z)(\mathrm{p})
$$

não muda de sinal em $\tilde{\Omega}$.

Demonstração. Basta mostrar que $f(x, y)=\operatorname{det}(D Z)(x, y),(x, y) \in \tilde{\Omega}$, não muda de sinal uma vizinhança de cada ponto de conjunto característico $\Sigma$ de $L$.

Seja $\mathrm{p}_{0} \in \Sigma$. Existem um difeomorfismo $F: U \rightarrow(-a, a)^{2}, a>0$, de classe $C^{1}$, onde $U$ é uma vizinhança aberta de $\mathrm{p}$, e um polinômio de grau $1, H$, tal que

$$
Z(x, y)=\left(H \circ Z_{\phi} \circ F\right)(x, y), \text { para todo }(x, y) \in U,
$$

onde

$$
Z_{\phi}(s, t)=s+i \phi(s, t),(s, t) \in(-a, a)^{2},
$$

$\operatorname{com} \phi:(-a, a)^{2} \rightarrow \mathbb{R}$ uma função de classe $C^{1}$. 
Segue de (2.17) que

$$
\operatorname{det}(D Z)(x, y)=\mu \operatorname{det}\left(D Z_{\phi}\right)(F(x, y)) \operatorname{det}(F)(x, y),(x, y) \in U,
$$

onde $\mu \in \mathbb{R}$ é uma constante diferente de zero. Como

$$
\operatorname{det}\left(D Z_{\phi}\right)(s, t)=\phi_{t}(s, t),(s, t) \in(-a, a)^{2}
$$

para estudar o sinal de $f$ em $U$, basta estudar o sinal de $\phi_{t}$ em $(-a, a)^{2}$.

Como $Z$ é injetiva, temos $Z_{\phi}:(-a, a)^{2} \rightarrow \mathbb{C}$ injetiva. Assim, para cada $s \in(-a, a)$ fixado, a função

$$
(-a, a) \ni t \mapsto \phi(s, t)
$$

é injetiva. Então, para cada $s \in(-a, a)$ fixado, a função

$$
(-a, a) \ni t \mapsto \phi_{t}(s, t)
$$

não muda de sinal e não se anula em intervalos, i.e., para cada $s \in(-a, a)$, o conjunto

$$
\Gamma_{s}=\left\{t \in(-a, a) ; \phi_{t}(s, t)=0\right\}
$$

não possui intervalos abertos. Então, os conjuntos

$$
A^{+}=\left\{s \in(-a, a) ; \sup _{t} \phi_{t}(s, t)>0\right\}
$$

e

$$
A^{-}=\left\{s \in(-a, a) ; \inf _{t} \phi_{t}(s, t)<0\right\}
$$

são conjuntos disjuntos e $K=(-a, a) \backslash\left(A^{+} \cup A^{-}\right)$é um conjunto fechado tal que $\phi_{t}=0 \mathrm{em}$ $K \times(-a, a)$.

Note que $K$ é o conjunto vazio. De fato, se $K \neq \emptyset$, então existe $s_{0} \in K$ tal que

$$
(-a, a) \ni t \rightarrow \phi_{t}\left(s_{0}, t\right)
$$

é a função identicamente nula, ou seja, $\Gamma_{s_{0}}=(-a, a)$. Contradição.

Daí, concluímos que $(-a, a)^{2}$ é a união dos abertos disjuntos $A^{+} \times(-a, a)$ e $A^{-} \times(-a, a)$. Como $(-a, a)^{2}$ é conexo, segue que $A^{+}=\emptyset$ ou $A^{-}=\emptyset$. Sem perda de generalidade podemos supor que $A^{-}=\emptyset$. Assim, temos $A^{+}=(-a, a)$, isto é, $(-a, a)^{2}=A^{+} \times(-a, a)$. Segue daí que

$$
\phi_{t} \geq 0 \mathrm{em}(-a, a)^{2}
$$

Portanto $f$ não muda de sinal em $U$ e assim concluímos a demonstração. 


\subsection{Transversalidade de uma curva em relação a campo}

Esta seção é inspirada em um lema de (MEZIANI, 2011).

Seja $L=A \partial_{x}+B \partial_{y}$ um campo vetorial definido em um aberto $\tilde{\Omega}$ de $\mathbb{R}^{2}$.

Definição 10. Seja $\Gamma$ uma variedade mergulhada fechada de dimensão 1 em $\Omega$. Dizemos que $\Gamma$ é transversal a $L$ se

$$
i^{*} \omega \neq 0
$$

onde $\omega=B d x-A d y$ (é uma 1-forma ortogonal a $L=A \partial_{x}+B \partial_{y}$ ) e $i: \Gamma \rightarrow \Omega$ é a aplicação de inclusão.

Observação 18. A expressão (2.18) significa:

$$
\omega(\mathrm{p}, v)=B(\mathrm{p}) v_{1}-A(\mathrm{p}) v_{2} \neq 0
$$

para todo $(\mathrm{p}, v) \in T_{\mathrm{p}} \Gamma \operatorname{com}(\mathrm{p}, v) \neq(\mathrm{p}, 0)$ e $v=\left(v_{1}, v_{2}\right)$

Suponha que $L$ é um campo hipocomplexo em $\tilde{\Omega}$ e $Z: \tilde{\Omega} \rightarrow \mathbb{C}$ é uma integral primeira global, de classe $C^{1+\varepsilon}, 0 \leq \varepsilon<1$, de $L$ que é um homeomorfismo sobre sua imagem.

Seja $j: \mathbb{C} \rightarrow \mathbb{R}^{2}$ a aplicação de identificação, i.e., $j(z)=(\Re(z), \mathfrak{I}(z))$. Definimos $\tilde{Z}$ : $\tilde{\Omega} \rightarrow \mathbb{C}$ por $\tilde{Z}=Z \circ j$. Temos que $\tilde{Z}(\tilde{\Omega})$ é um aberto de $\mathbb{R}^{2}$ e $\tilde{Z}: \tilde{\Omega} \rightarrow \tilde{Z}(\tilde{\Omega})$ é de classe $C^{1+\varepsilon}$ e um homeomorfismo.

Lema 3. Sejam $G \in C^{\alpha}(\Gamma), 0<\alpha<1$, e $\Gamma$ uma variedade mergulhada fechada de dimensão 1 de $\tilde{\Omega}$, de classe $C^{1+\varepsilon}$, transversal a $L$. Então

1. $\tilde{Z}(\Gamma)$ é uma variedade mergulhada fechada de dimensão 1 de $\tilde{Z}(\tilde{\Omega}) \subset \mathbb{R}^{2}$ e de classe $C^{1+\varepsilon}$;

2. $\tilde{G}: \tilde{Z}(\Gamma) \rightarrow \mathbb{C}$ dada por $\tilde{G}=G \circ \tilde{Z}^{-1}$ é de classe $C^{\alpha}$ em $\tilde{Z}(\Gamma)$.

Demonstração. Seja $(\mathrm{p}, v) \in T_{\mathrm{p}} \Gamma \operatorname{com}(\mathrm{p}, v) \neq(\mathrm{p}, 0)$ e $v=\left(v_{1}, v_{2}\right)$. Temos

$$
\omega(\mathrm{p}, v)=B(\mathrm{p}) v_{1}-A(\mathrm{p}) v_{2} \neq 0
$$

Como $L=A \partial_{x}+B \partial_{y}$ em $\tilde{\Omega}$, pela Proposição 4 (página 33) podemos escrever

$$
A=\lambda \partial_{y} Z \text { e } B=-\lambda \partial_{x} Z
$$

$\operatorname{com} \lambda \in C(\tilde{\Omega})$ e $|\lambda|>0$ em $\tilde{\Omega}$. Assim, de (2.19) temos

$$
\lambda(\mathrm{p})\left\{-\partial_{x} Z(\mathrm{p}) v_{1}-\partial_{y} Z(\mathrm{p}) v_{2}\right\} \neq 0,
$$

isto é,

$$
\partial_{x} Z(\mathrm{p}) v_{1}+\partial_{y} Z(\mathrm{p}) v_{2} \neq 0
$$


Daí,

$$
\mathfrak{R}\left(\partial_{x} Z(\mathrm{p}) v_{1}+\partial_{y} Z(\mathrm{p}) v_{2}\right) \neq 0 \text { ou } \mathfrak{I}\left(\partial_{x} Z(\mathrm{p}) v_{1}+\partial_{y} Z(\mathrm{p}) v_{2}\right) \neq 0
$$

Ou seja,

$$
\left[\begin{array}{ll}
\partial_{x} \mathfrak{R}(Z)(\mathrm{p}) & \partial_{y} \mathfrak{R}(Z)(\mathrm{p}) \\
\partial_{x} \mathfrak{I}(Z)(\mathrm{p}) & \partial_{y} \mathfrak{I}(Z)(\mathrm{p})
\end{array}\right]\left[\begin{array}{c}
v_{1} \\
\\
v_{2}
\end{array}\right] \neq 0, \operatorname{para}(\mathrm{p}, v) \in T_{\mathrm{p}} \Gamma \operatorname{com}(\mathrm{p}, v) \neq(\mathrm{p}, 0) .
$$

Segue daí que

$$
i^{*} \tilde{Z}:=\tilde{Z} \circ i: \Gamma \rightarrow \mathbb{R}^{2}
$$

é uma imersão, onde $i: \Gamma \rightarrow \tilde{\Omega}$ é a aplicação de inclusão. Como $Z$ é um homeomorfismo temos que $i^{*} \tilde{Z}$ é um mergulho. Logo, $\tilde{Z}(\Gamma)$ é uma variedade mergulhada de $\tilde{Z}(\tilde{\Omega}) \subset \mathbb{R}^{2}$, o que prova o item 1. Além disso,

$$
W:=i^{*} \tilde{Z}=j \circ Z \circ i: \Gamma \rightarrow \tilde{Z}(\Gamma)
$$

é um difeomorfismo. Daí, $W^{-1}: \tilde{Z}(\Gamma) \rightarrow \Gamma$ é uma aplicação de classe $C^{1}$. Seja

$$
M: \tilde{Z}(\Gamma) \rightarrow \mathbb{R}^{2}
$$

dada por $M(\mathrm{q})=W^{-1}(\mathrm{q})$. Como $\tilde{Z}(\Gamma)$ é uma variedade mergulhada fechada de $\mathbb{R}^{2}$, pelo Teorema de Tietze diferenciável (veja (LIMA, 2007)) $M$ possui uma extensão $C^{1}$ para todo o $\mathbb{R}^{2}$, isto é, existe

$$
M_{\circ}: \mathbb{R}^{2} \rightarrow \mathbb{R}^{2}
$$

de classe $C^{1}$ tal que $\left.M_{\circ}\right|_{\tilde{Z}(\Gamma)}=W^{-1}$. Como $\tilde{Z}(\Gamma)$ é compacta, a aplicação $M_{\circ}$ é Lipschitz sobre $\tilde{Z}(\Gamma)$, isto é, segue que existe uma constate $K$ tal

$$
\left|M_{\circ}\left(\mathrm{q}_{1}\right)-M_{\circ}\left(\mathrm{q}_{2}\right)\right| \leq K\left|\mathrm{q}_{1}-\mathrm{q}_{2}\right|, \mathrm{q}_{1}, \mathrm{q}_{2} \in \tilde{Z}(\Gamma) .
$$

Segue daí que,

$$
\left|\tilde{Z}^{-1}\left(\mathrm{q}_{1}\right)-\tilde{Z}^{-1}\left(\mathrm{q}_{2}\right)\right| \leq K\left|\mathrm{q}_{1}-\mathrm{q}_{2}\right|, \mathrm{q}_{1}, \mathrm{q}_{2} \in \tilde{Z}(\Gamma) .
$$

Fazendo o cálculo da condição de Hölder para $\tilde{G}$ temos

$$
\left|\tilde{G}\left(\mathrm{q}_{1}\right)-\tilde{G}\left(\mathrm{q}_{2}\right)\right| \leq\left|G\left(\tilde{Z}^{-1}\left(\mathrm{q}_{1}\right)\right)-G\left(\tilde{Z}^{-1}\left(\mathrm{q}_{2}\right)\right)\right| \leq C_{G}\left|\tilde{Z}^{-1}\left(\mathrm{q}_{1}\right)-\tilde{Z}^{-1}\left(\mathrm{q}_{2}\right)\right|^{\alpha} \leq C_{G} K^{\alpha}\left|\mathrm{q}_{1}-\mathrm{q}_{2}\right|^{\alpha},
$$

para todos $\mathrm{q}_{1}, \mathrm{q}_{2} \in \tilde{Z}(\Gamma)$. Portanto, $\tilde{G} \in C^{\alpha}(\tilde{Z}(\Gamma))$. Assim concluímos a demonstração do item 2. 

CAPÍTULO

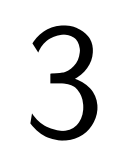

(2)

\section{A CLASSE DOS CAMPOS VETORIAIS EM \\ ESTUDO}

Neste capítulo vamos estabelecer a classe de campos vetoriais complexos que estudaremos neste texto.

\subsection{Uma classe de campos hipocomplexos}

No capítulo anterior vimos que campos hipocomplexos possuem boas propriedades, em especial a existência de integral primeira global. A seguir estabeleceremos uma classe de campos vetoriais hipocomplexos que será objeto de estudo desta tese. Um campo $L$ definido no aberto $\tilde{\Omega} \subset \mathbb{R}^{2}$ pertence a classe $\mathscr{X}(\tilde{\Omega})$ se satisfaz as seguintes condições:

(i) $L$ é um campo não singular de classe $C^{\varepsilon}, 0<\varepsilon<1$, em $\Omega$;

(ii) O conjunto característico $\Sigma$ de $L$ é uma subvariedade mergulhada de dimensão 1 de $\Omega$, de classe $C^{1+\varepsilon}$, com um número finito de componentes conexas;

(iii) $L$ é de classe $C^{1}$ em $\tilde{\Omega} \backslash \Sigma$;

(iv) Para cada $\mathrm{p} \in \tilde{\Omega} \backslash \Sigma$, existe uma integral primeira $Z: U \rightarrow \mathbb{C}$ de $L$, de classe $C^{2}$, com $U \subset \tilde{\Omega} \backslash \Sigma$ aberto e $p \in U$, tal que $Z: U \rightarrow Z(U) \subset \mathbb{C}$ é um homeomorfismo;

(v) Para cada $\mathrm{p} \in \Sigma$, existem uma integral primeira $Z: U \rightarrow \mathbb{C}$ de $L$, com $U \subset \tilde{\Omega}$ uma vizinhança aberta de p, tal que $Z: U \rightarrow Z(U) \subset \mathbb{C}$ é um homeomorfismo que satisfaz $Z_{x y}, Z_{y x} \in C(U)$ e $Z_{x y}=Z_{y x}$ em $U$, e coordenadas locais $(s, t)$ em $U$, de classe $C^{2}$ centradas em $\mathrm{p}$, tal que nas novas variáveis a integral primeira $Z$ se escreve como

$$
Z_{\sigma, \beta}(s, t)=s+i\left(\frac{t|t|^{\sigma}}{\sigma+1}+\beta(s)\right),
$$


onde $\beta$ é uma função a valores reais de classe $C^{2+\varepsilon}$ que depende apenas de $s$ e $\sigma \geq \varepsilon$ é uma constante. Além disso, $Z$ satisfaz a seguinte condição: se $L=A \partial_{x}+B \partial_{y}$ em $\Omega$, então a função $\lambda: U \rightarrow \mathbb{C}$ definida por

$$
\lambda(x, y)= \begin{cases}\frac{A(x, y)}{Z_{y}(x, y)}, & \text { se } Z_{y}(x, y) \neq 0 \\ -\frac{B(x, y)}{Z_{x}(x, y)}, & \text { se } Z_{x}(x, y) \neq 0\end{cases}
$$

é de classe $C^{1}$ em $U$.

Observação 19. Parte das condições do item (v) acima estão relacionadas com a regularidade do campo. Se $L$ é $C^{2}$-hipocomplexo (Definição 7, página 35 ) e o seu conjunto característico $\Sigma$ é uma variedade mergulhada de dimensão 1 de $\tilde{\Omega}$, de classe $C^{1+\varepsilon}$, segue da Proposição 6 (página 36) que qualquer integral primeira de $L, Z: U \rightarrow \mathbb{C}$, com $U \subset \tilde{\Omega}$ aberto, é de classe $C^{2}$. Logo $Z_{x y}$, $Z_{y x} \in C(U)$ e $Z_{x y}=Z_{y x}$ em $U$ (Teorema de Schwarz). Além disso, com essas hipóteses sobre $L$, a condição sobre $\lambda$ (dada em (3.2)) ser de classe $C^{1}$ se verifica trivialmente. A função $\lambda$ está relacionada com a Proposição 4 (página 33).

Para o número real $\sigma>0$ considere o campo vetorial complexo

$$
L_{\sigma}=\frac{\partial}{\partial y}-i|y|^{\sigma} \frac{\partial}{\partial x}
$$

definido em $\mathbb{R}^{2}$. Temos que $L_{\sigma}$ possui integral primeira global

$$
Z_{\sigma}(x, y)=x+i \frac{y|y|^{\sigma}}{\sigma+1}
$$

que é um homeomorfismo de $\mathbb{R}^{2}$ sobre $\mathbb{C}$. Assim o campo $L_{\sigma}$ é um campo vetorial complexo $C^{1+\sigma}$-hipocomplexo em $\mathbb{R}^{2}$. Observe, se $0<\sigma<1$ temos que $Z_{\sigma}$ não é de classe $C^{2}$ e $L_{\sigma}$ não é de classe $C^{1}$. O campo vetorial $L_{\sigma}$ pertence a classe $\mathscr{X}\left(\mathbb{R}^{2}\right)$. Na verdade, $L_{\sigma}$ é mais que um exemplo, $L_{\sigma}$ é o campo modelo da teoria desenvolvida nesta tese. (Veja a Proposição 11, página 53). O campo $L_{\sigma}$ também será chamando de campo padrão ao decorrer dos capítulos a seguir. O campo $L_{\sigma}$ faz parte de uma família de campos vetoriais complexos indexada a um parâmetro real $\sigma \geq 0, \operatorname{com} L_{0}=-2 i \partial / \partial \bar{z}$, onde $\partial / \partial \bar{z}$ é o operador de Cauchy-Riemann. Em essência, o que demonstraremos nesta tese é que grande parte dos resultados clássicos de resolubilidade e problemas de contorno para $L_{0}$ (isto é, para $\partial / \partial \bar{z}$ ) se generalizam para os outros campos $L_{\sigma}$, $\sigma>0$, da família.

A classe $\mathscr{X}$ engloba campos de baixa regularidade, isto é, campos que não são de classe $C^{\infty}$. Mesmo assim, é possível estudar equações diferenciais, envolvendo campos de $\mathscr{X}$, 
no sentido das distribuições. A Proposição a seguir sustenta essa afirmação como veremos na Observação 20.

Antes da proposição, vamos ressaltar um fato importante. Temos que a classe $\mathscr{X}$ é composta por campos $C^{1+\varepsilon}$-hipocomplexos. Seja $L$ um campo definido em $\Omega$ e pertencente a classe $\mathscr{X}(\tilde{\Omega})$. Pela Proposição 7 (página 37 ) o campo $L$ possui uma integral primeira global $Z$ : $\tilde{\Omega} \rightarrow \mathbb{C}$, de classe $C^{1+\varepsilon}$, que é um homeomorfismo sobre sua imagem, isto é, $Z: \tilde{\Omega} \rightarrow Z(\tilde{\Omega}) \subset \mathbb{C}$ é um homeomorfismo.

Proposição 9. Seja $L$ um campo vetorial complexo definido em $\Omega$ pertencente à classe $\mathscr{X}(\tilde{\Omega})$. Suponha que $Z: \tilde{\Omega} \rightarrow \mathbb{C}$, de classe $C^{1+\varepsilon}$, é uma integral primeira global de $L$ que é um homeomorfismo sobre a imagem. Então, $Z$ satisfaz $Z_{x y}, Z_{y x} \in C(\tilde{\Omega})$ e $Z_{x y}=Z_{y x}$ em $\tilde{\Omega}$. Além disso, existe $\lambda_{Z}: \tilde{\Omega} \rightarrow \mathbb{C}$ de classe $C^{1}$, que não se anula em $\tilde{\Omega}$, tal que

$$
L=\lambda_{Z} H_{Z} \operatorname{em} \tilde{\Omega}
$$

onde $H_{Z}=Z_{y} \partial_{x}-Z_{x} \partial_{y}$ é o Hamiltoniano de $Z$.

Demonstração. Seja $Z: \tilde{\Omega} \rightarrow \mathbb{C}$ uma integral primeira de $L$, de classe $C^{1+\varepsilon}$, que é um homeomorfismo sobre sua imagem. Pela Proposição 4 (página 33) existe $\lambda_{Z} \in C^{\varepsilon}(\tilde{\Omega})$ tal que vale a igualdade

$$
L=\lambda_{Z}\left\{Z_{y} \partial_{x}-Z_{x} \partial_{y}\right\}=\lambda_{Z} H_{Z} \operatorname{em} \tilde{\Omega}
$$

Pela Proposição 6 (página 36) e pela condição (iv) temos que $Z$ é de classe $C^{2}$ em $\tilde{\Omega} \backslash \Sigma$. Em particular, $Z_{x y}, Z_{y x} \in C(\tilde{\Omega} \backslash \Sigma)$ e pelo Teorema de $\operatorname{Schwarz} Z_{x y}=Z_{y x}$ em $\tilde{\Omega} \backslash \Sigma$. Segue da expressão de $L$ acima e da condição (iii) que $\lambda_{Z}$ é de classe $C^{1}$ em $\tilde{\Omega} \backslash \Sigma$.

Agora, seja $\mathrm{p} \in \Sigma$. Pela condição (v), para $\delta>0$ suficientemente pequeno, existem $F: U_{\delta} \rightarrow F\left(U_{\delta}\right) \subset \mathbb{R}^{2}$, com $U_{\delta}=B(\mathrm{p}, \delta)$, um difeomorfismo de classe $C^{2}$ tal que $F\left(\Sigma \cap U_{\delta}\right)=$ $(-a, a) \times\{0\}, \operatorname{com} a>0, \mathrm{e}$

$$
Z_{\sigma, \beta}(s, t)=s+i\left(\frac{t|t|^{\sigma}}{\sigma+1}+\beta(s)\right) \operatorname{em} F\left(U_{\delta}\right)
$$

com $\sigma>0$ constante e $\beta$ a valores reais de classe $C^{2+\varepsilon}$, tais que

$$
Z^{\bullet}:=Z_{\sigma, \beta} \circ F \text { em } U_{\delta}
$$

é uma integral primeira de $L$ que satisfaz

$$
Z_{x y}^{\bullet}, Z_{y x}^{\bullet} \in C\left(U_{\delta}\right) \text { e } Z_{x y}^{\bullet}=Z_{y x}^{\bullet} \text { em } U_{\delta}
$$

Pela Proposição 6, existe um biholomorfismo $h: Z^{\bullet}\left(U_{\delta}\right) \rightarrow Z\left(U_{\delta}\right)$ tal que

$$
Z=h \circ Z^{\bullet} \operatorname{em~} U_{\delta}
$$


Assim, $Z_{x y}, Z_{y x} \in C\left(U_{\delta}\right)$ e $Z_{x y}=Z_{y x}$ em $U_{\delta}$. Além disso, pela condição (v), existe $\lambda_{Z} \bullet: U_{\delta} \rightarrow \mathbb{C}$ de classe $C^{1}$ tal que

$$
L=\lambda_{Z} \cdot H_{Z} \cdot \operatorname{em} U_{\delta}
$$

Segue de (3.4) que

$$
H_{Z}=h^{\prime}\left(Z^{\bullet}\right) H_{Z} \cdot \operatorname{em} U_{\delta}
$$

Logo, por (3.3), (3.5) e (3.6), vale a seguinte igualdade

$$
\lambda_{Z} h^{\prime}\left(Z^{\bullet}\right) H_{Z} \cdot=\lambda_{Z} \cdot H_{Z} \cdot \operatorname{em} U_{\delta}
$$

Como $H_{Z}$ é um campo vetorial não singular temos

$$
\lambda_{Z} h^{\prime}\left(Z^{\bullet}\right)=\lambda_{Z} \cdot \operatorname{em} U_{\delta}
$$

Como as funções $h^{\prime} \circ Z^{\bullet}$ e $\lambda_{Z} \bullet$ são de classe $C^{1}$ em $U_{\delta}$ e $h^{\prime} \circ Z^{\bullet}$ não se anula em $U_{\delta}$ da igualdade acima temos que $\lambda_{Z}$ é de classe $C^{1}$ em $U_{\delta}$. Pela arbitrariedade de $\mathrm{p} \in \Sigma$ segue que $\lambda_{Z}$ é de classe $C^{1}$ numa vizinhança de $\Sigma$ em $\tilde{\Omega}$. Como $\lambda_{Z}$ é de classe $C^{1}$ em $\tilde{\Omega} \backslash \Sigma$, como visto acima, temos que $\lambda_{Z}$ é de classe $C^{1}$ em $\Omega$. Assim concluímos a demonstração.

Observação 20. Seja $L$ um campo vetorial pertencente a classe $\mathscr{X}(\tilde{\Omega})$ e seja $Z: \tilde{\Omega} \rightarrow \mathbb{C}$ uma integral primeira global de $L$ de classe $C^{1+\varepsilon}$ em $\tilde{\Omega}$. Pela Proposição 9 podemos escrever

$$
L=\lambda_{Z} H_{Z} \operatorname{em} \tilde{\Omega}
$$

$\operatorname{com} \lambda_{Z} \in C^{1}(\tilde{\Omega})$ e $\left|\lambda_{Z}\right|>0$ em $\tilde{\Omega}$. Pela Observação 10 (página 33) temos que $L u, H_{Z} u \in \mathscr{D}_{1}^{\prime}(\tilde{\Omega})$ para $u \in \mathscr{D}_{0}^{\prime}(\tilde{\Omega})$. Assim, podemos buscar soluções distribucionais em $\mathscr{D}_{0}^{\prime}(\tilde{\Omega})$ para equações envolvendo $L$, por exemplo, a equação $L u=f$ em $\tilde{\Omega}$, com $f \in L^{p}(\tilde{\Omega}), p>1$. Além disso, se $\Omega \subset \tilde{\Omega}$ é um aberto limitado com $\bar{\Omega} \subset \tilde{\Omega}$, estudar a equação,

$$
L u=f \operatorname{em} \Omega,
$$

em $\mathscr{D}_{0}^{\prime}(\Omega), \operatorname{com} f \in L^{p}(\Omega), p>1$, equivale, no sentido das distribuições, a estudar a equação

$$
H_{Z} u=\frac{f}{\lambda_{Z}} \operatorname{em} \Omega
$$

Esta relação é um instrumento importante no estudo da equação $L u=f$. Outro ponto a se observar é que a igualdade $Z_{x y}=Z_{y x}$ em $\tilde{\Omega}$ (Proposição 9) tem importância em variais partes deste texto, por exemplo na representação de soluções - veja a demonstração da Proposição 16, página 76 - e também no Teorema 5 (página 78) devido à seguinte relação entre $H_{Z}$ e seu transposto ${ }^{t} H_{Z}$ :

$$
{ }^{t} H_{Z}=-H_{Z}+\left\{Z_{y x}-Z_{x y}\right\}=-H_{Z}
$$


Proposição 10. Suponha que o campo vetorial $L=A \partial / \partial x+B \partial / \partial y$ definido em $\Omega$ pertença à classe $\mathscr{X}(\tilde{\Omega})$. O expoente $\sigma>0$ que aparece na condição (v) é intrínseco do campo $L$ no seguinte sentido. Para cada componente conexa de $\Sigma$, existe um único $\sigma>0$ para o qual vale (3.1).

Demonstração. Seja $\mathrm{p} \in \Sigma$ e sejam $\mathscr{U}_{j}, j=1,2$, tais que $\mathrm{p} \in \mathscr{U}_{1} \cap \mathscr{U}_{2}$ e vale a condição (v), isto é, a menos de uma mudança de coordenadas de classe $C^{2}$ em cada $\mathscr{U}_{j}$ temos que

$$
Z_{\sigma_{j}, \beta_{j}}(s, t)=s+i\left(\frac{t|t|^{\sigma_{j}}}{\sigma_{j}+1}+\beta_{j}(s)\right)
$$

é uma integral primeira de $L$ (com $\sigma_{j}$ e $\beta_{j}$ descritos na condição (v)). Então

$$
\mathfrak{I}(A \bar{B})(x, y)=\left|\rho_{j}(x, y)\right|^{\sigma_{j}} g_{j}(x, y),(x, y) \in \mathscr{U}_{j}
$$

$\operatorname{com} \rho_{j}: \mathscr{U}_{j} \rightarrow \mathbb{R}$ de classe $C^{2} \operatorname{com} d \rho_{j} \neq 0$ em $\mathscr{U}_{j}$ e $g_{j}$ de classe $C^{1}$ em $\mathscr{U}_{j} \operatorname{com} g_{j} \neq 0$ em $\mathscr{U}_{j}$.

Como o conjunto característico $\Sigma$ é uma subvariedade mergulhada de dimensão 1 em $\tilde{\Omega}$, de classe $C^{1+\varepsilon}$, existe um difeomorfismo de classe $C^{1+\varepsilon}$

$$
\Phi:(-\varepsilon, \varepsilon)^{2} \rightarrow V
$$

onde $\mathrm{p} \in V \subset \mathscr{U}_{1} \cap \mathscr{U}_{2}$, tal que

$$
\Phi((-\varepsilon, \varepsilon) \times\{0\})=\Sigma \cap V \text { e } \Phi(0,0)=\text { p. }
$$

Temos por (3.7) que

$$
\left|\rho_{1}(x, y)\right|^{\sigma_{1}} g_{1}(x, y)=\left|\rho_{2}(x, y)\right|^{\sigma_{2}} g_{2}(x, y),(x, y) \in V
$$

Daí,

$$
\left|\vartheta_{1}(s, t)\right|^{\sigma_{1}} \varphi_{1}(s, t)=\left|\vartheta_{2}(s, t)\right|^{\sigma_{2}} \varphi_{2}(s, t),(s, t) \in(-\varepsilon, \varepsilon)^{2}
$$

onde $\vartheta_{j}=\left(\rho_{j} \circ \Phi\right)$ e $\varphi_{j}=\left(g_{j} \circ \Phi\right), j=1,2$. Temos que $d \vartheta_{j} \neq 0$ em $(-\varepsilon, \varepsilon)^{2}$ e $\vartheta_{j}(s, 0)=0$ para $s \in(-\varepsilon, \varepsilon)$. Temos também que $\varphi_{j} \neq 0$ em $(-\varepsilon, \varepsilon)^{2}$.

Segue que $\partial_{t} \vartheta_{j}(s, 0) \neq 0$ para $s \in(-\varepsilon, \varepsilon)$. Assim, existe $0<\varepsilon^{\prime} \leq \varepsilon$, tal que

$$
\partial_{t} \vartheta_{j}(s, t) \neq 0,(s, t) \in\left(-\varepsilon^{\prime}, \varepsilon^{\prime}\right)^{2}
$$

Por outro lado, podemos escrever

$$
\vartheta_{j}(s, t)=t f_{j}(s, t),(s, t) \in\left(-\varepsilon^{\prime}, \varepsilon^{\prime}\right)^{2}
$$

onde

$$
f_{j}(s, t)=\int_{0}^{1} \partial_{t} \vartheta_{j}(s, t \eta) d \eta,(s, t) \in\left(-\varepsilon^{\prime}, \varepsilon^{\prime}\right)^{2}
$$


Por (3.9), segue que $f_{j}(s, t) \neq 0$ para todo $(s, t) \in\left(-\varepsilon^{\prime}, \varepsilon^{\prime}\right)^{2}$. Por (3.8) e (3.10) temos

$$
\left|t f_{1}(s, t)\right|^{\sigma_{1}} \varphi_{1}(s, t)=\left|t f_{2}(s, t)\right|^{\sigma_{2}} \varphi_{2}(s, t),(s, t) \in\left(-\varepsilon^{\prime}, \varepsilon^{\prime}\right)^{2}
$$

Assim temos a igualdade

$$
|t|^{\sigma_{1}} \mu_{1}(s, t)=|t|^{\sigma_{2}} \mu_{2}(s, t),(s, t) \in\left(-\varepsilon^{\prime}, \varepsilon^{\prime}\right)^{2}
$$

$\operatorname{com} \mu_{j}=\left|f_{j}\right|^{\sigma_{j}} \varphi_{j}, j=1,2$, em $(-\varepsilon, \varepsilon)^{2}$. Note que $\mu_{j}(s, t) \neq 0$, para todo $(s, t) \in\left(-\varepsilon^{\prime}, \varepsilon^{\prime}\right)^{2}$. Daí,

$$
\frac{|t|^{\sigma_{1}}}{|t|^{\sigma_{2}}}=\frac{\mu_{2}(s, t)}{\mu_{1}(s, t)}<\infty,(s, t) \in\left(-\varepsilon^{\prime}, \varepsilon^{\prime}\right)^{2} .
$$

Segue que $\sigma_{1} \geq \sigma_{2}$. Da mesma forma, temos

$$
\frac{|t|^{\sigma_{2}}}{|t|^{\sigma_{1}}}=\frac{\mu_{1}(s, t)}{\mu_{2}(s, t)}<\infty,(s, t) \in\left(-\varepsilon^{\prime}, \varepsilon^{\prime}\right)^{2}
$$

o que implica $\sigma_{1} \leq \sigma_{2}$. Portanto, $\sigma_{1}=\sigma_{2}$.

Segue daí que a cada componente conexa de $\Sigma$ podemos associar um único número $\sigma>0$ tal que vale (3.1), concluindo a demonstração desta proposição.

Observação 21. O expoente $\sigma$ de (3.1) associado à componente conexa $\Sigma^{\prime}$ de $\Sigma$, é chamado de tipo de $L$ em $\Sigma^{\prime}$. A palavra tipo vem de "tipo finito $k$ " ( $k$ inteiro positivo). Falaremos mais sobre isso na Seção 3.3, página 56. Veja a definição de tipo finito na Seção 2.3.1, página 37.

Exemplo 5. Temos que os campos da classe $\mathscr{X}$ são $C^{1+\varepsilon}$-hipocomplexos. Mas nem todo campo $C^{1+\varepsilon}$-hipocomplexo pertence a classe $\mathscr{X}$.

O campo vetorial (de tipo infinito)

$$
L=\frac{\partial}{\partial y}-i \frac{e^{-\frac{1}{|y|}}}{y^{2}} \frac{\partial}{\partial x}
$$

é de classe $C^{\infty}$, com conjunto característico $\Sigma=\left\{(x, y) \in \mathbb{R}^{2} ; y=0\right\}$. Além disso, $L$ é hipocomplexo com integral primeira global

$$
Z(x, y)=x+i \frac{y}{|y|} e^{-\frac{1}{|y|}}
$$

No entanto, a condição (v) não é satisfeita, pois $e^{-\frac{1}{|y|}}$ decai mais rapidamente que $|y|^{\sigma}$ para qualquer $\sigma>0$.

Vamos terminar essa seção com uma versão do teorema de representação de soluções para soluções contínuas da equação $L u=0$, para $L$ pertencente a classe $\mathscr{X}(\tilde{\Omega})$. 
Teorema 2. Seja $L$ um campo vetorial complexo, definido no aberto $\tilde{\Omega} \subset \mathbb{R}^{2}$, pertencente à classe $\mathscr{X}(\tilde{\Omega})$. Seja $Z_{U}: U \rightarrow \mathbb{C}$ de classe $C^{1+\varepsilon}$, uma integral primeira de $L$, que é um homeomorfismo sobre sua imagem, isto é, $Z_{U}: U \rightarrow Z_{U}(U) \subset \mathbb{C}$ é um homeomorfismo. Se $u \in C(U)$ é solução de $L u=0$ em $U$, então existe uma função holomorfa $h: Z_{U}(U) \rightarrow \mathbb{C}$ tal que $u=h \circ Z_{U}$ em $U$.

Demonstração. Considere a função contínua $h: Z_{U}(U) \rightarrow \mathbb{C}$ dada por

$$
h=u \circ Z_{U}^{-1} .
$$

Temos pela condição (iv) (página 47) e pela Proposição 6 (página 36) que $Z_{U}$ é de classe $C^{2}$ em $U \backslash(U \cap \Sigma)$. Temos que $L$ é elítico em $U \backslash(U \cap \Sigma)$ e pela condição (iii) (página 47) o campo $L$ é de classe $C^{1}$ em $U \backslash(U \cap \Sigma)$. Segue da Proposição 5 (página 34) que $h$ é holomorfa em $U \backslash(U \cap \Sigma)$. Como uma argumentação análoga a usada na demonstração da Proposição 6, usando o teorema da extensão de funções holomorfas (Teorema 23, página 132), podemos demonstrar que $h$ é holomorfa.

No caso em que $L$ é $C^{\infty}$-hipocomplexo o teorema de representação de soluções vale para soluções distribucionais do campo. Veja o Teorema 3 na página 57.

\subsection{Normalização}

A condição (v) estabelece, juntamente com o Teorema 2, a forma local das integrais primeiras do campo numa vizinhança de cada ponto do característico. Tal forma local é dada por (3.1), isto é,

$$
Z_{\sigma, \beta}(s, t)=s+i\left(\frac{t|t|^{\sigma}}{\sigma+1}+\beta(s)\right)
$$

onde $\beta$ é uma função de classe $C^{2+\varepsilon}$ a valores reais. A próxima proposição elimina $\beta$ da forma local através de conjugação, deixando assim, a expressão da integral primeira determinada pelo tipo $\sigma$.

A seguinte proposição pode ser encontrada em (CAMPANA; SILVA; MEZIANI, 2017).

Proposição 11. Seja $L$ um campo vetorial complexo, definido no aberto $\tilde{\Omega}$ de $\mathbb{R}^{2}$, pertencente a classe $\mathscr{X}(\tilde{\Omega})$. Para cada $\mathrm{p} \in \Sigma$, existe uma vizinhança aberta $U$ de $\mathrm{p}$ tal que $U \backslash \Sigma$ consiste em duas componentes conexas $U^{+}$e $U^{-}$e coordenadas locais $\left(x^{+}, y^{+}\right)$(respectivamente $\left(x^{-}, y^{-}\right)$) centradas em $\mathrm{p}$ tal que $L$ é um múltiplo do seguinte campo vetorial em $U^{+}$(respectivamente $\left.U^{-}\right)$:

$$
L_{\sigma}=\frac{\partial}{\partial y^{ \pm}}-i\left|y^{ \pm}\right| \sigma \frac{\partial}{\partial x^{ \pm}}
$$

com a integral primeira

$$
Z_{\sigma}\left(x^{ \pm}, y^{ \pm}\right)=x^{ \pm}+i \frac{y^{ \pm}\left|y^{ \pm}\right| \sigma}{\sigma+1} \operatorname{em~} U^{ \pm}
$$


Demonstração. Seja $\mathrm{p} \in \Sigma$ e seja $Z$ uma integral primeira de $L$ definida em uma vizinhança de $\mathrm{p}$. Pela condição (v) (página 47) e pelo Teorema 2 podemos começar a normalização a partir de $Z_{\sigma, \beta}$, dada em (3.11), numa vizinhança da origem de $\mathbb{R}^{2}, \operatorname{com} \beta(0)=0$. Seja $D_{\delta}$ um disco, de raio $\delta$ suficientemente pequeno, centrado em $(0,0)$, de tal forma que $D_{\delta}$ esteja no domínio de definição de $Z_{\sigma, \beta}$, e tal que $\Sigma \subset\left\{(s, t) \in \mathbb{R}^{2} ; t=0\right\}$ divide $D_{\delta}$ em dois semidiscos

$$
D_{\delta}^{+}=\left\{(s, t) \in \mathbb{R}^{2} ; t>0\right\} \text { e } D_{\delta}^{-}=\left\{(s, t) \in \mathbb{R}^{2} ; t<0\right\} .
$$

Note que $Z_{\sigma, \beta}\left(D^{+}\right)$e $Z_{\sigma, \beta}\left(D^{-}\right)$são abertos simplesmente conexos de $\mathbb{C}$ que compartilham a curva $\gamma_{\delta}=\{s+i \beta(s) ; s \in(-\delta, \delta)\}$, de classe $C^{2+\varepsilon}$, em seu bordo. Seja $0<\delta_{1}<\delta$. Seja $V^{ \pm} \subset Z_{\sigma, \beta}\left(D^{ \pm}\right)$aberto simplesmente conexo com bordo suave de classe $C^{2+\varepsilon}$ tal que $\gamma_{\delta_{1}}$ faz parte de seu bordo. Pelo Teorema da Aplicação de Riemann (veja o Teorema 21, página 131) podemos encontrar uma função holomorfa

$$
H^{ \pm}: V^{ \pm} \longrightarrow H^{ \pm}\left(V^{ \pm}\right) \subset \mathbb{C}
$$

que envia a curva de bordo $\gamma_{\delta_{1}}$ sobre o eixo real e $H^{ \pm}$se estende a um difeomorfismo de classe $C^{2}$ sobre $\partial V^{ \pm}$. Em particular, $H^{ \pm}$está definida numa vizinhança de 0 em $\mathbb{C}$. Podemos supor que $H^{ \pm}(0)=0$ e $H^{ \pm}\left(V^{ \pm}\right) \subset\{\zeta \in \mathbb{C} ; \pm \mathfrak{I}(\zeta)>0\}$. Assim a função

$$
Z_{\sigma, \beta}^{ \pm}=H^{ \pm} \circ Z_{\sigma, \beta}
$$

esta bem definida em $D_{\delta_{2}}, 0<\delta_{2} \leq \delta_{1}$, com regularidade $C^{1}$ e, além disso, $Z_{\sigma, \beta}^{ \pm}$é uma integral primeira de $L$ em $D_{\delta_{2}}^{ \pm}$. Mais ainda, $Z_{\sigma, \beta}^{ \pm}$satisfaz

$$
\mathfrak{I} Z_{\sigma, \beta}^{ \pm}(s, 0)=0, s \in\left(-\delta_{2}, \delta_{2}\right)
$$

Segue daí que

$$
\mathfrak{I} Z_{\sigma, \beta}^{ \pm}(s, t)=t|t|^{\sigma} \psi^{ \pm}(s, t)
$$

para uma função $\psi^{ \pm}$, de classe $C^{1}, \operatorname{com} \psi^{ \pm}(0,0)>0$. De fato, para $\zeta=\xi+i \eta$, temos

$$
\begin{gathered}
\mathfrak{I}\left(Z_{\sigma, \beta}^{ \pm}\right)(s, t)=\mathfrak{I}\left(Z_{\sigma, \beta}^{ \pm}\right)(s, 0)+\int_{0}^{1} \mathfrak{I}\left\{\left(\partial_{\zeta} H^{ \pm}-\partial_{\zeta} H^{ \pm}\right)\left(Z_{\sigma, \beta}(s, t \rho)\right) i|t \rho|^{\sigma} t\right\} d \rho \\
=t|t|^{\sigma} \int_{0}^{1} \partial_{\eta} \mathfrak{I}\left(H^{ \pm}\right)\left(Z_{\sigma, \beta}(s, t \rho)\right)|\rho|^{\sigma} d \rho=: t|t|^{\sigma} \psi^{ \pm}(s, t) .
\end{gathered}
$$

Como $\pm \mathfrak{I}\left(Z_{\sigma, \beta}^{ \pm}\right)(s, t) \geq 0$ para $(s, t) \in D_{\delta_{2}}^{ \pm}$, segue da igualdade (3.14), que $\psi^{ \pm} \geq 0$. Assim, basta verificar que $\psi^{ \pm}(0,0) \neq 0$. Temos,

$$
\psi^{ \pm}(0,0)=\int_{0}^{1} \partial_{\eta} \mathfrak{I}\left(H^{ \pm}\right)\left(Z_{\sigma, \beta}(0,0)\right)|\rho|^{\sigma} d \rho=\frac{1}{\sigma+1} \partial_{\eta} \mathfrak{I}\left(H^{ \pm}\right)(0) .
$$

Suponha por contradição que $\partial_{\eta} \mathfrak{I}\left(H^{ \pm}\right)(0)=0$. Temos assim que

$$
\operatorname{det}\left(D H^{ \pm}\right)(0)=-\partial_{\xi} \mathfrak{I}\left(H^{ \pm}\right)(0) \partial_{\eta} \mathfrak{R}\left(H^{ \pm}\right)(0)
$$


Por outro lado, temos que

$$
f(s):=\mathfrak{I}\left(H^{ \pm}\right)(\gamma(s))=0, \forall s \in\left(-\delta_{2}, \delta_{2}\right),
$$

onde, relembrando, $\gamma(s)=s+i \beta(s), \operatorname{com} \gamma(0)=0$. Daí, temos $f^{\prime}(0)=0$, ou seja,

$$
\partial_{\xi} \mathfrak{I}\left(H^{ \pm}\right)(\gamma(0))+\partial_{\eta} \mathfrak{I}\left(H^{ \pm}\right)(\gamma(0)) \beta^{\prime}(0)=0 .
$$

Como $\partial_{\eta} \mathfrak{I}\left(H^{ \pm}\right)(0)=0$, temos $\partial_{\xi} \mathfrak{I}\left(H^{ \pm}\right)(0)=0$. Assim, em (3.15), temos

$$
\operatorname{det}\left(D H^{ \pm}\right)(0)=0
$$

que é uma contradição, pois $H^{ \pm}$é um difeomorfismo numa vizinhança de 0. Portanto, $\partial_{\eta} \mathfrak{I}\left(H^{ \pm}\right)(0) \neq$ 0 e concluímos a verificação de que $\psi^{ \pm}>0 \mathrm{em} D_{\delta_{2}}$ (reduzindo $\delta_{2}$ se necessário). Assim, podemos escrever

$$
\mathfrak{I} Z_{\sigma, \beta}^{ \pm}(s, t)=\frac{t|t|^{\sigma}}{\sigma+1}\left\{\chi^{ \pm}\right\}^{\sigma+1}(s, t)
$$

em $D_{\delta_{2}}$, onde $\chi^{ \pm}:=(\sigma+1)^{\frac{1}{\sigma+1}}\left\{\psi^{ \pm}\right\}^{\frac{1}{\sigma+1}}$.

Com um argumento análogo ao usado acima, mostramos que $\left(x^{ \pm}, y^{ \pm}\right)$dadas por

$$
x^{ \pm}=\mathfrak{R} Z_{\sigma, \beta}^{ \pm}(s, t), \quad y^{ \pm}=t \chi^{ \pm}(s, t)
$$

são coordenadas locais de classe $C^{1}$ em $D_{\delta_{3}}$, para $0<\delta_{3}<\delta_{2}$. Nessas coordenadas $Z_{\sigma, \beta}$ tem o formato desejado.

Demonstramos que existe uma vizinhança $U$ de $\mathrm{p}, \mathrm{p} \in \Sigma$, tal que $U \backslash \Sigma$ consiste em duas componentes conexas $U^{+}$e $U^{-}$, isto é,

$$
U=(U \cap \Sigma) \cup U^{+} \cup U^{-},
$$

e existem difeomorfismos $F^{ \pm}: \bar{U} \rightarrow[-\delta, \delta]^{2}, G^{ \pm}: \overline{Z_{\sigma}\left((-\delta, \delta)^{2}\right)} \rightarrow \overline{V^{ \pm}}$, onde $V^{ \pm}$é um aberto de $\mathbb{C}$, tais que podemos escrever

$$
Z(x, y)=\left(G^{ \pm} \circ Z_{\sigma} \circ F^{ \pm}\right)(x, y), \forall(x, y) \in U
$$

onde

$$
Z_{\sigma}(s, t)=s+i \frac{t|t|^{\sigma}}{\sigma+1},(s, t) \in(-\delta, \delta)^{2},
$$

com $\left.G^{ \pm}\right|_{Z_{\sigma}\left((-\delta, \delta) \times I^{ \pm}\right)}: Z_{\sigma}\left((-\delta, \delta) \times I^{ \pm}\right) \rightarrow \mathbb{C}$ holomorfa injetiva, onde $I^{+}=(0, \delta)$ e $I^{-}=$ $(-\delta, 0)$.

Usando (3.16) podemos relacionar o Hamiltoniano de $Z$ e com o Hamiltoniano de $Z_{\sigma}$ e obter o formato desejado do campo $L$ em $(-\delta, \delta) \times I^{ \pm}$, a menos de um múltiplo contínuo, através da mudança de coordenadas dada por $\left(F^{ \pm}\right)^{-1}$.

A seguir temos corolários que exploram a conjugação local por difeomorfismos. 


\section{Corolário 1. (Corolário da demonstração da Proposição 11) Seja}

$$
Z_{\sigma, \beta}(s, t)=s+i\left(\frac{t|t|^{\sigma}}{\sigma+1}+\beta(s)\right),(s, t) \in V
$$

onde $V$ é uma vizinhança de 0 em $\mathbb{R}^{2}, \sigma>0$ é uma constante e $\beta$ é uma função a valores reais de classe $C^{2+\varepsilon}$. Reduzindo $V$ se necessário, existem um difeomorfismo de classe $C^{1}, F: W \rightarrow V, W$ vizinhança de $(0,0)$ em $\mathbb{R}^{2}$, e um difeomorfismo de classe $C^{1}, G: Z_{\sigma, \beta}(V) \rightarrow G\left(Z_{\sigma, \beta}(V)\right) \subset \mathbb{C}$, tais que

$$
Z_{\sigma}=G \circ Z_{\sigma, \beta} \circ F \text { em } W
$$

onde

$$
Z_{\sigma}(x, y)=x+i \frac{y|y|^{\sigma}}{\sigma+1} \text { em } W
$$

O corolário abaixo também é consequência da demonstração da proposição acima e será usado para fazer estimativas do operador $T_{Z} f$ na seção 5.1.

Corolário 2. Seja $L$ um campo vetorial complexo definido no aberto $\tilde{\Omega}$ pertencente a classe $\mathscr{X}(\tilde{\Omega})$. Seja p $\in \Sigma$ e $Z: U \rightarrow \mathbb{C}, U \subset \tilde{\Omega}$ aberto, uma integral primeira de classe $C^{1+\varepsilon}$ de $L$ que é um homeomorfismo sobre a imagem, com $\mathrm{p} \in U$. Reduzindo $U$ se necessário, existem $\sigma>0$ uma constante e difeomorfismos $F: \bar{U} \rightarrow[-\delta, \delta]^{2}$ e $G: \overline{Z_{\sigma}\left((-\delta, \delta)^{2}\right)} \rightarrow \bar{V}$, onde $V$ é um aberto de $\mathbb{C}$, tais que podemos escrever

$$
Z(x, y)=\left(G \circ Z_{\sigma} \circ F\right)(x, y), \forall(x, y) \in U
$$

onde

$$
Z_{\sigma}(s, t)=s+i \frac{t|t|^{\sigma}}{\sigma+1},(s, t) \in(-\delta, \delta)^{2}
$$

A constante $\sigma>0$ é o tipo de campo $L$ na componente conexa de $\Sigma$ onde p foi tomado. Veja a Proposição 10, página 51, e a Observação 21, página 52.

Referente ao corolário acima, ressaltamos que $Z_{\sigma}$ (dada em (3.19)) não é uma integral primeira de $L$ em outras coordenadas, uma vez que $G$ não é holomorfa em $U$.

\subsection{Observações acerca do caso hipoelítico}

Seja $L$ um campo não singular suave definido em $\tilde{\Omega}$, isto é,

$$
L=A \partial_{x}+B \partial_{y} \operatorname{em} \tilde{\Omega},
$$


$\operatorname{com} A, B \in C^{\infty}(\tilde{\Omega}),|A|+|B|>0$ em $\tilde{\Omega}$.

Suponha que $L$ é $C^{\infty}$-hipocomplexo, isto é, $L$ possui um sistema completo de integrais primeiras de classe $C^{\infty}$ que são homeomorfismos. Isto significa que para todo $\mathrm{p} \in \tilde{\Omega}$ existe uma função de classe $C^{\infty}, Z: \mathscr{U} \rightarrow \mathbb{C}$, definida em um conjunto aberto $\mathscr{U} \subset \tilde{\Omega}, \mathrm{p} \in \mathscr{U}$, tal que $d Z \neq 0, L Z=0, Z: \mathscr{U} \rightarrow Z(\mathscr{U}) \subset \mathbb{C}$ é um homeomorfismo. (Veja a Definição 7).

Definição 11. Dizemos que $L$ é hipoelítico em $\Omega$ quando se verifica a seguinte condição para todo aberto não vazio $\mathscr{V}$ de $\Omega$ : se $L u=f \in C^{\infty}(\mathscr{V})$ onde $u \in \mathscr{D}^{\prime}(\mathscr{V})$, então $u \in C^{\infty}(\mathscr{V})$.

Proposição 12. As seguintes afirmações são equivalentes:

(1) L é hipoelítico;

(2) $L$ é $C^{\infty}$-hipocomplexo.

Veja (BERGAMASCO; CORDARO; HOUNIE, 1988). Esta proposição é consequência do estudo das condições (P) e (Q) descritas em (TREVES, 1971).

Segue do teorema da Fórmula de Aproximação de Baouendi-Treves uma versão do Teorema 2, página 53, para $L$ hipoelítico:

Teorema 3. Seja $L$ um campo hipoelítico em $\tilde{\Omega}$. Seja $Z: \mathscr{U} \rightarrow \mathbb{C}$, integral primeira de $L$, de classe $C^{\infty}$, definida em um conjunto aberto $\mathscr{U} \subset \tilde{\Omega}$, tal que $Z: \mathscr{U} \rightarrow Z(\mathscr{U}) \subset \mathbb{C}$ é um homeomorfismo. Se $u \in \mathscr{D}^{\prime}(\mathscr{U})$ é solução de $L u=0$ em $\mathscr{U}$, então existe uma função holomorfa $h: Z(\mathscr{U}) \rightarrow \mathbb{C}$ tal que $u=h \circ Z$ em $\mathscr{U}$.

Demonstração. Como dito acima, esse resultado segue do Teorema da Fórmula de Aproximação de Baouendi-Treves. Veja em (TREVES, 1992) e (BERHANU; CORDARO; HOUNIE, 2008).

Como consequência, temos a versão da Proposição 7, página 37, para o caso hipoelítico.

Proposição 13. Seja $L$ um campo vetorial hipoelítico não singular definido no aberto conexo $\Omega$ de $\mathbb{R}^{2}$. Então $L$ possui uma integral primeira global em $\Omega$ que é um homeomorfismo sobre sua imagem. Mais precisamente, existe uma função

$$
Z: \tilde{\Omega} \rightarrow Z(\tilde{\Omega}) \subset \mathbb{C}
$$

de classe $C^{\infty}$, tal que $Z$ é um homeomorfismo, $L Z=0$ e $d Z \neq 0$ em $\tilde{\Omega}$.

Demonstração. Veja (BERGAMASCO; CORDARO; HOUNIE, 1988). A demonstração é inteiramente análoga à da Proposição 7. Veja também (CAMPANA, 2013). 
Note que nesta seção, até neste momento, não colocamos restrições ao conjunto característico.

Agora, vamos analisar uma classe de campos hipoelíticos que está contida na classe $\mathscr{X}$ (definida na página 47).

Seja $L$ um campo hipoelítico definido no aberto $\tilde{\Omega}$. Suponha que o conjunto característico de $L, \Sigma$, é uma subvariedade mergulhada de dimensão 1 de $\Omega$ de classe $C^{\infty}$ com um número finito de componentes conexas, $\Sigma_{1}, \cdots, \Sigma_{N}$. Para $j \in\{1, \ldots, N\}$, suponha que $L$ tenha tipo finito $k_{j}$ em todo $\mathrm{p} \in \Sigma_{j}$. Isto é, $L$ tem tipo uniforme $k_{j}$ em $\Sigma_{j}$.

Seja p $\in \Sigma_{j}$. Seja $Z$ uma integral primeira de $L$, de classe $C^{\infty}$, definida numa vizinhança de $p$ e injetiva. Existem coordenadas locais, de modo que nessas coordenadas, $Z$ está definida numa vizinhança da origem e se escreve como

$$
Z(s, t)=s+i \phi(s, t)
$$

onde $\phi$ é uma função a valores reais. A hipótese de tipo uniforme $k_{j}$ implica que

$$
\phi(s, t)=\left(t^{k_{j}+1} \psi(s, t)+\beta(s)\right)
$$

onde $\psi$ e $\beta$ são funções suaves e $\psi(0,0) \neq 0$ (veja em (BERHANU; CORDARO; HOUNIE, 2008)). Como se trata de uma análise local, podemos supor sem perda de generalidade, que $\psi$ não muda de sinal. Considere as coordenadas locais

$$
x=s \text { e } y=c t\left(k_{j}+1\right)^{\frac{1}{k_{j}+1}}|\psi(s, t)|^{\frac{1}{k_{j}+1}}
$$

$\operatorname{com} c=\operatorname{sgn}(\psi)($ sinal de $\psi)$. Nas coordenadas $(x, y)$ a integral primeira $Z$ se escreve na forma

$$
Z_{k_{j}, \beta}(x, y)=x+i\left(\frac{y^{k_{j}+1}}{k_{j}+1}+\beta(x)\right) .
$$

Em particular, como $Z_{k_{j}, \beta}$ é injetiva, segue que $k_{j}$ é par.

Concluímos assim a seguinte proposição:

Proposição 14. Seja $L$ um campo hipoelítico não singular definido em um aberto $\tilde{\Omega}$ de $\mathbb{R}^{2}$, com seu conjunto característico $\Sigma$ sendo uma subvariedade mergulhada de dimensão 1 de classe $C^{\infty} \mathrm{e}$ com um número finito de componentes conexas. Se $L$ tem tipo finito uniforme (o qual deve ser par) em cada componente conexa de $\Sigma$, então $L$ pertence a classe $\mathscr{X}(\tilde{\Omega})$ (definida na página 47 ). 
CAPÍTULO

\section{LEMAS TÉCNICOS}

Neste capítulo vamos provar alguns lemas técnicos que iremos usar nos próximos capítulos.

Lema 4. Seja $0<\delta<R, 0<\tau<1, a \in \mathbb{R}$, e $q^{\prime}>2-\tau$. Então, existe uma constante $M\left(\tau, q^{\prime}\right)>0$ tal que

$$
I=\int_{0}^{2 \pi} \int_{\delta}^{R} \frac{d r d \theta}{|a+r \sin \theta|^{\tau} r^{q^{\prime}-1}} \leq \frac{M\left(\tau, q^{\prime}\right)}{\delta^{q^{\prime}+\tau-2}}
$$

Demonstração. Esta demonstração é uma adaptação da demonstração de um lema de (MEZIANI, 2005).

Para provar esse lema é suficiente considerar $a \geq 0$. De fato, suponha que $a \leq 0$. Então, se $\theta^{\prime}=-\theta+2 \pi$, temos

$$
I=\int_{0}^{2 \pi} \int_{\delta}^{R} \frac{d r d \theta}{|a+r \sin \theta|{ }^{\tau} r^{q^{\prime}-1}}=\int_{2 \pi}^{0} \int_{\delta}^{R} \frac{-d r d \theta^{\prime}}{\left|a-r \sin \theta^{\prime}\right|{ }^{\tau} r^{q^{\prime}-1}}=\int_{0}^{2 \pi} \int_{\delta}^{R} \frac{d r d \theta^{\prime}}{\left|-a+r \sin \theta^{\prime}\right|^{\tau} r^{q^{\prime}-1}}
$$

A demonstração está dividida em três casos.

Caso 1: $a=0$. Temos,

$$
\begin{aligned}
& I=\int_{0}^{2 \pi} \int_{\delta}^{R} \frac{d r d \theta}{|r \sin \theta|^{\tau} r^{q^{\prime}-1}}=\int_{0}^{2 \pi} \int_{\delta}^{R} \frac{d r d \theta}{|\sin \theta|^{\tau} r^{q^{\prime}+\tau-1}}=\int_{0}^{2 \pi} \frac{d \theta}{|\sin \theta|^{\tau}} \int_{\delta}^{R} \frac{d r}{r^{q^{\prime}+\tau-1}} \\
& \leq C(\tau) \int_{\delta}^{R} \frac{d r}{r^{q^{\prime}+\tau-1}}=\frac{C(\tau)}{2-q^{\prime}-\tau}\left\{R^{2-q^{\prime}-\tau}-\delta^{2-q^{\prime}-\tau}\right\}=\frac{C(\tau)}{q^{\prime}+\tau-2}\left\{\left(\frac{1}{\delta}\right)^{q^{\prime}+\tau-2}-\left(\frac{1}{R}\right)^{q^{\prime}+\tau-2}\right\} \\
& \leq \frac{C(\tau)}{q^{\prime}+\tau-2}\left(\frac{1}{\delta}\right)^{q^{\prime}+\tau-2}
\end{aligned}
$$

onde

$$
C(\tau)=\int_{0}^{2 \pi} \frac{d \theta}{|\sin \theta|^{\tau}}<\infty
$$


Caso 2: $0<a<\delta$. Seja $\theta_{1} \in(-\pi / 2,0)$ tal que $\sin \theta_{1}=-a / r$. Então,

$$
\int_{-\pi / 2}^{\pi / 2} \frac{d \theta}{|a+r \sin \theta|^{\tau}}=\int_{-\pi / 2}^{\pi / 2} \frac{\left|\theta-\theta_{1}\right|^{\tau}}{r^{\tau}\left|\sin \theta-\sin \theta_{1}\right|^{\tau}} \frac{d \theta}{\left|\theta-\theta_{1}\right|^{\tau}} \leq \frac{A(\tau)}{r^{\tau}\left|\cos \theta_{1}\right|^{\tau}},
$$

para uma constante positiva $A(\tau)$. De fato, basta notar que a função $g:[-\pi / 2,0] \times[-\pi / 2, \pi / 2] \rightarrow$ $\mathbb{R}$ dada por

$$
g\left(\alpha_{1}, \alpha\right)=\cos \left(\alpha_{1}\right) \frac{\left|\alpha-\alpha_{1}\right|}{\left|\sin \alpha-\sin \alpha_{1}\right|}
$$

é limitada. É possível obter uma desigualdade semelhante para integral sobre o intervalo $[\pi / 2,3 \pi / 2]$. O passo seguinte é substituir $\left|\cos \theta_{1}\right|$ por $r / \sqrt{r^{2}-a^{2}}$, e obter

$$
\int_{0}^{2 \pi} \frac{d \theta}{|a+r \sin \theta|^{\tau}} \leq \frac{A(\tau)}{\left(r^{2}-a^{2}\right)^{\frac{\tau}{2}}}
$$

Então

$$
\begin{gathered}
I \leq A(\tau) \int_{\delta}^{R} \frac{d r}{\left(r^{2}-a^{2}\right)^{\frac{\tau}{2}} r^{q^{\prime}-1}} \leq A(\tau) \int_{\delta}^{R} \frac{d r}{\left(r^{2}-\delta^{2}\right)^{\frac{\tau}{2}} r^{q^{\prime}-1}} \\
\leq A(\tau) \delta^{2-q^{\prime}-\tau} \int_{1}^{\frac{R}{\delta}} \frac{d \rho}{\left(\rho^{2}-1\right)^{\frac{\tau}{2}} \rho^{q^{\prime}-1}} \leq A(\tau) \delta^{2-q^{\prime}-\tau} \int_{1}^{\infty} \frac{d \rho}{\left(\rho^{2}-1\right)^{\frac{\tau}{2}} \rho^{q^{\prime}-1}}=: A(\tau) \delta^{2-q^{\prime}-\tau} H .
\end{gathered}
$$

Podemos escrever

$$
H=\int_{1}^{2} \frac{d \rho}{\left(\rho^{2}-1\right)^{\frac{\tau}{2}} \rho^{q^{\prime}-1}}+\int_{2}^{\infty} \frac{d \rho}{\left(\rho^{2}-1\right)^{\frac{\tau}{2}} \rho^{q^{\prime}-1}}=: H_{1}+H_{2} .
$$

Temos

Para $\mathrm{H}_{2}$ temos

$$
H_{1}=\int_{1}^{2} \frac{d \rho}{\left(\rho^{2}-1\right)^{\frac{\tau}{2}} \rho^{q^{\prime}-1}} \leq \int_{1}^{2} \frac{d \rho}{\left(\rho^{2}-1\right)^{\frac{\tau}{2}}} \leq \frac{1}{1-\tau} .
$$

$$
H_{2}=\int_{2}^{\infty} \frac{d \rho}{\left(\rho^{2}-1\right)^{\frac{\tau}{2}} \rho^{q^{\prime}-1}} \leq \int_{2}^{\infty} \frac{d \rho}{(\rho-1)^{\tau+q^{\prime}-1}}=\frac{1}{q^{\prime}+\tau-2} .
$$

Portanto, existe uma constante $M\left(q^{\prime}, \tau\right)>0$ tal que $I \leq M\left(q^{\prime}, \tau\right) \delta^{2-q^{\prime}-\tau}$.

Caso 3: $\delta \leq a$. Temos

$$
I \leq \int_{\delta}^{a} \int_{0}^{2 \pi} \frac{d \theta d r}{|a+r \sin \theta|^{\tau} r^{q^{\prime}-1}}+\int_{a}^{\infty} \int_{0}^{2 \pi} \frac{d \theta d r}{|a+r \sin \theta|^{\tau} r^{q^{\prime}-1}}=: I_{1}+I_{2}
$$

A estimativa da integral $I_{2}$ segue do caso anterior.

Assim, resta estimar $I_{1}$.

Note que, para $\delta \leq r \leq a$ temos $|a+r \sin \theta| \geq a-r$. Então,

$$
I_{1} \leq \int_{\delta}^{a} \int_{0}^{2 \pi} \frac{d \theta d r}{(a-r)^{\tau} r^{q^{\prime}-1}}=2 \pi \int_{\delta}^{a} \frac{d \theta d r}{(a-r)^{\tau} r^{q^{\prime}-1}}=: 2 \pi J
$$

Seja $u=a-r$. Então,

$$
J=\int_{0}^{a-\delta} \frac{d u}{u^{\tau}(a-u)^{q^{\prime}-1}} .
$$

Note que $a=\delta$ implica $J=0$. Assim, podemos supor $a>\delta$. Vamos dividir o estudo da estimativa para $J$ em casos. 
61

Caso 3.1: $q^{\prime}-1<1$.

$J=a^{2-q^{\prime}-\tau} \int_{0}^{\frac{a-\delta}{a}} \frac{d \rho}{\rho^{\tau}(1-\rho)^{q^{\prime}-1}} \leq \delta^{2-q^{\prime}-\tau} \int_{0}^{\frac{a-\delta}{a}} \frac{d \rho}{\rho^{\tau}(1-\rho)^{q^{\prime}-1}} \leq \delta^{2-q^{\prime}-\tau} \int_{0}^{1} \frac{d \rho}{\rho^{\tau}(1-\rho)^{q^{\prime}-1}}$.

Como $0<\tau<1$ e $0<q^{\prime}-1<1$, temos

$$
C\left(q^{\prime}, \tau\right)=\int_{0}^{1} \frac{d \rho}{\rho^{\tau}(1-\rho)^{q^{\prime}-1}}<\infty .
$$

Portanto $J \leq C\left(q^{\prime}, \tau\right) \delta^{2-q^{\prime}-\tau}$.

Case 3.2: $q^{\prime}-1>1$

Temos $q^{\prime}-2>0$. Então,

$$
J=\int_{0}^{a-\delta} \frac{d u}{u^{\tau}(a-u)^{q^{\prime}-2}(a-u)} \leq \frac{1}{\delta^{q^{\prime}-2}} \int_{0}^{a-\delta} \frac{d u}{u^{\tau}(a-u)} .
$$

Observe que

$$
\int_{0}^{a-\delta} \frac{d u}{u^{\tau}(a-u)}=\frac{1}{(a-\delta)^{\tau}} \sum_{j=0}^{\infty} \frac{1}{j+1-\tau}\left(\frac{a-\delta}{a}\right)^{j+1}=: \frac{1}{(a-\delta)^{\tau}} S .
$$

Note que,

$$
\frac{1}{j+1-\tau} \leq \frac{2}{j+1}, \forall j \geq 1 \mathrm{e} \frac{1}{1-\tau} \leq 2 \Leftrightarrow \tau \leq \frac{1}{2}
$$

Se $\tau \leq \frac{1}{2}$, então

$$
S \leq 2 \sum_{j=0}^{\infty} \frac{1}{j+1}\left(\frac{a-\delta}{a}\right)^{j+1} .
$$

Se $\tau \geq \frac{1}{2}$, então $K(\tau):=\frac{1}{1-\tau}-2 \geq 0$, e podemos escrever

$$
\begin{gathered}
S \leq \frac{1}{1-\tau}\left(\frac{a-\delta}{a}\right)+2 \sum_{j=1}^{\infty} \frac{1}{j+1}\left(\frac{a-\delta}{a}\right)^{j+1} \\
=\frac{1}{1-\tau}\left(\frac{a-\delta}{a}\right)-2\left(\frac{a-\delta}{a}\right)+2 \sum_{j=0}^{\infty} \frac{1}{j+1}\left(\frac{a-\delta}{a}\right)^{j+1} \\
=K(\tau)\left(\frac{a-\delta}{a}\right)+2 \sum_{j=0}^{\infty} \frac{1}{j+1}\left(\frac{a-\delta}{a}\right)^{j+1} .
\end{gathered}
$$

Observe que, $\delta<a$ implica

$$
\frac{1}{(a-\delta)^{\tau}}\left(\frac{a-\delta}{a}\right)=\frac{(a-\delta)^{1-\tau}}{a} \leq \frac{a^{1-\tau}}{a}=\frac{1}{a^{\tau}} \leq \frac{1}{\delta^{\tau}} .
$$

Portanto, para $0<\tau<1$, temos

$$
\frac{1}{(a-\delta)^{\tau}} S \leq|K(\tau)| \frac{1}{\delta^{\tau}}+\frac{2}{(a-\delta)^{\tau}} \sum_{j=0}^{\infty} \frac{1}{j+1}\left(\frac{a-\delta}{a}\right)^{j+1} .
$$


Agora note que,

$$
\sum_{j=0}^{\infty} \frac{1}{j+1}\left(\frac{a-\delta}{a}\right)^{j+1}=-\ln \left(1-\frac{a-\delta}{a}\right)=-\ln \left(\frac{\delta}{a}\right)=\ln a-\ln \delta .
$$

Como $a>\delta$ podemos escrever $a=\delta(1+y)$ para um $y>0$. Então,

$$
\frac{\ln a-\ln \delta}{(a-\delta)^{\tau}}=\frac{1}{\delta^{\tau}} \frac{\ln (1+y)}{y^{\tau}} \leq \frac{1}{\delta^{\tau}} B(\tau)
$$

onde

$$
B(\tau):=\sup _{y>0} \frac{\ln (1+y)}{y^{\tau}}<\infty(\text { pois } 0<\tau<1) .
$$

Portanto,

$$
J=\frac{1}{\delta^{q^{\prime}-2}} \frac{1}{(a-\delta)^{\tau}} S \leq \frac{1}{\delta^{q^{\prime}-2}}\left\{|K(\tau)| \frac{1}{\delta^{\tau}}+2 \frac{1}{\delta^{\tau}} B(\tau)\right\}=\frac{C(\tau)}{\delta^{q^{\prime}+\tau-2}},
$$

onde $C(\tau)=|K(\tau)|+2 B(\tau)$. Assim,

$$
I_{1} \leq \frac{2 \pi C(\tau)}{\delta^{q^{\prime}+\tau-2}}
$$

Assim a demonstração do lema esta completa.

Como um caso particular do lema anterior temos o seguinte lema:

Lema 5 ((MEZIANI, 2005), Lema 3.1). Seja $0<\delta<R, 0<\tau<1, m>0$, e $0 \leq \gamma<R$. Então, existe uma constante $C(\tau)>0$ tal que

$$
\int_{\delta}^{R} \int_{0}^{2 \pi} \frac{d \theta d r}{|\gamma+r \sin \theta|^{\tau} r^{m+1}} \leq \frac{C(\tau)}{\delta^{m+\tau}}
$$

O próximo lema pode ser encontrado em (CAMPANA; SILVA; MEZIANI, 2017).

Lema 6. Seja $R>0,0<\tau<1, \gamma \in \mathbb{R}$, e $1<q<2-\tau$. Então, existe uma constante $M(q, \tau)>0$ tal que

$$
I=\int_{0}^{2 \pi} \int_{0}^{R} \frac{d r d \theta}{|\gamma+r \sin \theta|{ }^{\tau} r^{q-1}} \leq M(q, \tau) R^{2-\tau-q}
$$

Demonstração. Para provar este lema é suficiente considerar $\gamma \geq 0$.

Se $\gamma=0$, então

$$
I=\int_{0}^{2 \pi} \int_{0}^{R} \frac{d r d \theta}{|r \sin \theta|^{\tau} r^{q-1}}=\int_{0}^{2 \pi} \frac{d \theta}{|\sin \theta|^{\tau}} \int_{0}^{R} \frac{d r}{r^{\tau+q-1}}=C(\tau) \frac{R^{2-\tau-q}}{2-\tau-q},
$$

onde

$$
C(\tau)=\int_{0}^{2 \pi} \frac{d \theta}{|\sin \theta|^{\tau}}<\infty
$$


Agora, suponha $\gamma>0$. Seja $r=\rho \gamma$. Temos

$$
I=\gamma^{2-\tau-q} \int_{0}^{2 \pi} \int_{0}^{\frac{R}{\gamma}} \frac{d \rho d \theta}{|\rho \sin \theta+1|^{\tau} \rho^{q-1}} \doteq \gamma^{2-\tau-q} J
$$

Para estimar $J$, vamos considerar dois casos: $0<\gamma<R$ e $\gamma \geq R$.

Assuma que $0<\gamma<R$. Podemos escrever

$$
J=\int_{0}^{\pi} \int_{0}^{\frac{R}{\gamma}} \frac{d \rho d \theta}{|\rho \sin \theta+1|^{\tau} \rho^{q-1}}+\int_{\pi}^{2 \pi} \int_{0}^{\frac{R}{\gamma}} \frac{d \rho d \theta}{|\rho \sin \theta+1|^{\tau} \rho^{q-1}} \doteq J_{1}+J_{2} .
$$

Para $\theta \in[0, \pi]$ temos $\sin \theta \geq 0$; consequentemente, $\rho \sin \theta+1 \geq \rho \sin \theta$ para $\rho \in[0, R / \gamma]$. Assim,

$$
J_{1} \leq \int_{0}^{\pi} \int_{0}^{\frac{R}{\gamma}} \frac{d \rho d \theta}{|\rho \sin \theta|^{\tau} \rho^{q-1}} \leq \frac{C(\tau)}{2-\tau-q}\left(\frac{R}{\gamma}\right)^{2-\tau-q}
$$

Agora, iremos estimar $J_{2}$. Seja $\pi<\theta_{0}<3 \pi / 2$ tal que $-\sin \theta_{0}=\gamma / 2 R$. Podemos escrever

$$
\begin{aligned}
J_{2} & =\int_{\pi}^{\theta_{0}} \int_{0}^{\frac{R}{\gamma}} \frac{d \rho d \theta}{|\rho \sin \theta+1|^{\tau} \rho^{q-1}}+\int_{\theta_{0}}^{\frac{3 \pi}{2}} \int_{0}^{\frac{R}{\gamma}} \frac{d \rho d \theta}{|\rho \sin \theta+1|^{\tau} \rho^{q-1}} \\
& +\int_{\frac{3 \pi}{2}}^{3 \pi-\theta_{0}} \int_{0}^{\frac{R}{\gamma}} \frac{d \rho d \theta}{|\rho \sin \theta+1|^{\tau} \rho^{q-1}}+\int_{3 \pi-\theta_{0}}^{2 \pi} \int_{0}^{\frac{R}{\gamma}} \frac{d \rho d \theta}{|\rho \sin \theta+1|^{\tau} \rho^{q-1}} \\
& \doteq J_{2,1}+J_{2,2}+J_{2,3}+J_{2,4} .
\end{aligned}
$$

Seja $\varphi=-\theta+3 \pi$. Temos

$$
J_{2,3}=\int_{\frac{3 \pi}{2}}^{3 \pi-\theta_{0}} \int_{0}^{\frac{R}{\gamma}} \frac{d \rho d \theta}{|\rho \sin \theta+1|^{\tau} \rho^{q-1}}=\int_{\theta_{0}}^{\frac{3 \pi}{2}} \int_{0}^{\frac{R}{\gamma}} \frac{d \rho d \varphi}{|\rho \sin \varphi+1|^{\tau} \rho^{q-1}}=J_{2,2} .
$$

Também,

$$
J_{2,4}=\int_{3 \pi-\theta_{0}}^{2 \pi} \int_{0}^{\frac{R}{\gamma}} \frac{d \rho d \theta}{|\rho \sin \theta+1|^{\tau} \rho^{q-1}}=\int_{\pi}^{\theta_{0}} \int_{0}^{\frac{R}{\gamma}} \frac{d \rho d \varphi}{|\rho \sin \varphi+1|^{\tau} \rho^{q-1}}=J_{2,1} .
$$

Para estimar $J_{2,1}$. Note que

$$
0 \leq-\sin \theta \leq \frac{\gamma}{2 R}<\frac{1}{2}, \quad \text { para } \quad 0 \in\left[\pi, \theta_{0}\right]
$$

consequentemente, para $\theta \in\left[\pi, \theta_{0}\right]$ e $0<\rho<R / \gamma$, temos

$$
\rho|\sin \theta|<\frac{1}{2}<1+\rho \sin \theta .
$$

Assim,

$$
J_{2,1} \leq \int_{\pi}^{\theta_{0}} \int_{0}^{\frac{R}{\gamma}} \frac{d \rho d \theta}{\rho^{\tau}|\sin \theta|^{\tau} \rho^{q-1}} \leq \frac{C(\tau)}{4(2-\tau-q)}\left(\frac{R}{\gamma}\right)^{2-\tau-q}
$$


Para estimar $J_{2,2}$, considere $\varphi=\theta$ e $t=\rho \sin \theta+1$. Temos

$$
\begin{aligned}
J_{2,2} & =\int_{\theta_{0}}^{\frac{3 \pi}{2}} \int_{0}^{\frac{R}{\gamma}} \frac{d \rho d \theta}{|\rho \sin \theta+1|^{\tau} \rho^{q-1}}=\int_{\theta_{0}}^{\frac{3 \pi}{2}} \int_{1}^{\frac{R}{\gamma} \sin \varphi+1} \frac{1}{\sin \varphi} \frac{d t d \varphi}{|t|^{\tau}\left(\frac{t-1}{\sin \varphi}\right)^{q-1}} \\
& =\int_{\theta_{0}}^{\frac{3 \pi}{2}}|\sin \varphi|^{q-2}\left(\int_{\frac{R}{\gamma} \sin \varphi+1}^{1} \frac{d t}{|t|^{\tau}|t-1|^{q-1}}\right) d \varphi \\
& \leq \int_{\theta_{0}}^{\frac{3 \pi}{2}}|\sin \varphi|^{q-2}\left(\int_{-\frac{R}{\gamma}}^{1} \frac{d t}{|t|^{\tau}|t-1|^{q-1}}\right) d \varphi \cdot \leq C(q, \tau)\left(\frac{R}{\gamma}\right)^{2-\tau-q},
\end{aligned}
$$

para uma constante $C(q, \tau)>0$.

Assim, pelos cálculos acima, podemos encontrar uma constante $C_{1}(q, \tau)>0$ tal que

$$
J \leq C_{1}(q, \tau)\left(\frac{R}{\gamma}\right)^{2-\tau-q}
$$

Agora, assuma que $\gamma \geq R$. Neste caso, existe uma constante $C(q, \tau)>0$ tal que

$$
J=\int_{0}^{2 \pi} \int_{0}^{\frac{R}{\gamma}} \frac{d \rho d \theta}{|\rho \sin \theta+1|^{\tau} \rho^{q-1}} \leq \int_{0}^{2 \pi} \int_{0}^{\frac{R}{\gamma}} \frac{d \rho d \theta}{|1-\rho|^{\tau} \rho^{q-1}} \leq C(q, \tau)\left(\frac{R}{\gamma}\right)^{2-\tau-q}
$$

Finalmente, pelas estimativas (4.1), (4.2) e (4.3) existe uma constante $M(q, \tau)>0$ tal que

$$
I \leq M(q, \tau) R^{2-\tau-q}
$$

O próximo lema pode ser encontrado em (CAMPANA; SILVA; MEZIANI, 2017).

Lema 7. Seja $R>0, \gamma \geq 0,0<\tau<1,1 \leq q<2-\tau$, e $0 \leq \varphi \leq \pi$. Então, existe uma constante $C(q, \tau)>0$ tal que

$$
H=\int_{0}^{2 \pi} \int_{0}^{R} \frac{r d r d \theta}{|\gamma+r \sin (\theta+\varphi)|^{\tau} r^{q}\left|r e^{i \theta}-1\right|^{q}} \leq C(q, \tau) .
$$

Demonstração. Vamos dividir a prova em dois casos: $\gamma=0$ e $\gamma>0$.

Caso 1: $\gamma=0$. Neste caso podemos escrever

$$
H=\int_{0}^{2 \pi} \int_{0}^{R} \frac{d r d \theta}{|\sin (\theta+\varphi)|^{\tau} r^{q+\tau-1}\left|r e^{i \theta}-1\right|^{q}}=H_{1}+H_{2}+H_{3}
$$


com

$$
\begin{aligned}
& H_{1}=\int_{0}^{2 \pi} \int_{0}^{\frac{1}{2}} \frac{d r d \theta}{|\sin (\theta+\varphi)|^{\tau} r^{q+\tau-1}\left|r e^{i \theta}-1\right|^{q}}, \\
& H_{2}=\int_{0}^{2 \pi} \int_{\frac{1}{2}}^{\frac{3}{2}} \frac{d r d \theta}{|\sin (\theta+\varphi)|^{\tau} r^{q+\tau-1}\left|r e^{i \theta}-1\right|^{q}}, \\
& H_{3}=\int_{0}^{2 \pi} \int_{\frac{3}{2}}^{R} \frac{d r d \theta}{|\sin (\theta+\varphi)|^{\tau} r^{q+\tau-1}\left|r e^{i \theta}-1\right|^{q}} .
\end{aligned}
$$

0 tal que

Para $0<r<\frac{1}{2}$ temos $\left|r e^{i \theta}-1\right| \geq \frac{1}{2}$. Assim, podemos encontrar uma constante $C_{1}(q, \tau)>$

$$
H_{1} \leq 2^{q} \int_{0}^{2 \pi} \int_{0}^{\frac{1}{2}} \frac{d r d \theta}{|\sin (\theta+\varphi)|^{\tau} r^{q+\tau-1}} \leq C_{1}(q, \tau) .
$$

Para $\frac{1}{2}<r<\frac{3}{2}$ temos $\left(\frac{2}{3}\right)^{\tau+q}<\frac{1}{r^{\tau+q}}<2^{\tau+q}$, e daí,

$$
\begin{aligned}
H_{2} & \leq 2^{\tau+q} \int_{0}^{2 \pi} \int_{\frac{1}{2}}^{\frac{3}{2}} \frac{d r d \theta}{|\sin (\theta+\varphi)|^{\tau}\left|r e^{i \theta}-1\right|^{q}} \\
& =2^{\tau+q} \sum_{j=1}^{4} \int_{\frac{(j-1)}{2}}^{\frac{j \pi}{2}} \int_{\frac{1}{2}}^{\frac{3}{2}} \frac{d r d \theta}{|\sin (\theta+\varphi)|^{\tau}\left|r e^{i \theta}-1\right|^{q}} \doteq 2^{\tau+q} \sum_{j=1}^{4} H_{2, j} .
\end{aligned}
$$

Seja $0<\theta_{0}<\pi / 2$ tal que

$$
1-\frac{\theta^{2}}{2}<\cos \theta<1-\frac{\theta^{2}}{4}, \quad \text { para } 0<\theta<\theta_{0} .
$$

Então

$$
H_{2,1}=\int_{0}^{\theta_{0}} \int_{\frac{1}{2}}^{\frac{3}{2}} \frac{d r d \theta}{|\sin (\theta+\varphi)|^{\tau}\left|r e^{i \theta}-1\right|^{q}}+\int_{\theta_{0}}^{\frac{\pi}{2}} \int_{\frac{1}{2}}^{\frac{3}{2}} \frac{d r d \theta}{|\sin (\theta+\varphi)|^{\tau}\left|r e^{i \theta}-1\right|^{q}}=H_{2,1}^{1}+H_{2,1}^{2} .
$$

Para $0<\theta<\theta_{0}$ e $1 / 2 \leq r \leq 3 / 2$ temos

$$
\frac{(r-1)^{2}+\theta^{2}}{4} \leq(r-1)^{2}+\frac{\theta^{2}}{4} \leq(r-1)^{2}+r \frac{\theta^{2}}{2} \leq\left|r e^{i \theta}-1\right|^{2}
$$

e, consequentemente,

$$
\frac{1}{\left|r e^{i \theta}-1\right|^{2}} \leq \frac{4}{(r-1)^{2}+\theta^{2}}
$$

Assim,

$$
H_{2,1}^{1} \leq 2^{q} \int_{0}^{\theta_{0}} \int_{\frac{1}{2}}^{\frac{3}{2}} \frac{d r d \theta}{|\sin (\theta+\varphi)|^{\tau}\left[(r-1)^{2}+\theta^{2}\right]^{\frac{q}{2}}} \doteq 2^{q} J
$$

Suponha que $0 \leq \varphi \leq \pi / 2$. Então, $0<\theta+\varphi<\pi / 2+\theta_{0}<\pi$ e $|\sin (\theta+\varphi)| \geq C|\theta+\varphi|$, para uma constante $C>0$. Assim,

$$
\begin{aligned}
C^{\tau} J & \leq \int_{0}^{\theta_{0}} \int_{\frac{1}{2}}^{\frac{3}{2}} \frac{d r d \theta}{|\theta+\varphi|^{\tau}\left[(r-1)^{2}+\theta^{2}\right]^{\frac{q}{2}}} \leq \int_{D((0,1) ; 1)} \frac{d r d \theta}{|\theta+\varphi|^{\tau}\left[(r-1)^{2}+\theta^{2}\right]^{\frac{q}{2}}} \\
& =\int_{0}^{1} \int_{0}^{2 \pi} \frac{d \rho d t}{|\varphi+\rho \sin t|^{\tau} \rho^{q-1}} \leq M(q, \tau),
\end{aligned}
$$


onde a última desigualdade foi obtida pelo lema 6 (página 62).

Uma estimativa similar pode ser obtida no caso $\pi / 2<\varphi \leq \pi$. Portanto,

$$
\int_{0}^{\theta_{0}} \int_{\frac{1}{2}}^{\frac{3}{2}} \frac{d r d \theta}{|\sin (\theta+\varphi)|^{\tau}\left|r e^{i \theta}-1\right|^{q}} \leq \frac{2^{q} M(q, \tau)}{C^{\tau}} .
$$

Para $\theta_{0}<\theta<\frac{\pi}{2}$ temos

$$
\left|r e^{i \theta}-1\right|^{2} \geq 1-\cos \theta \geq 1-\cos \theta_{0}
$$

$\mathrm{e}$

$$
\begin{aligned}
H_{2,1}^{1} & =\int_{\theta_{0}}^{\frac{\pi}{2}} \int_{\frac{1}{2}}^{\frac{3}{2}} \frac{d r d \theta}{|\sin (\theta+\varphi)|^{\tau}\left|r e^{i \theta}-1\right|^{q}} \\
& \leq \frac{1}{\left(1-\cos \theta_{0}\right)^{\frac{q}{2}}} \int_{\theta_{0}}^{\frac{\pi}{2}} \frac{d \theta}{|\sin (\theta+\varphi)|^{\tau}} \leq \frac{C_{1}(\tau)}{\left(1-\cos \theta_{0}\right)^{\frac{q}{2}}}
\end{aligned}
$$

para uma constante $C_{1}(\tau)>0$. Segue de (4.5) e (4.6), que

$$
H_{2,1} \leq \frac{2^{q} M(q, \tau)}{C^{\tau}}+\frac{C_{1}(\tau)}{\left(1-\cos \theta_{0}\right)^{\frac{q}{2}}} .
$$

Para estimar $H_{2,2}$, vamos começar usando a mudança de variáveis $\theta=\alpha+\pi$ na integral para obter

$$
H_{2,2}=\int_{\frac{3 \pi}{2}}^{2 \pi} \int_{\frac{1}{2}}^{\frac{3}{2}} \frac{d r d \alpha}{|\sin (\alpha+\varphi)|^{\tau}\left|r e^{i \alpha}+1\right|^{q}} .
$$

Note que $\left|r e^{i \alpha}+1\right| \geq r \cos \alpha+1 \geq 1$, para $3 \pi / 2 \leq \alpha \leq 2 \pi$; assim,

$$
H_{2,2} \leq \int_{\frac{3 \pi}{2}}^{2 \pi} \frac{d \alpha}{|\sin (\alpha+\varphi)|^{\tau}}=C_{2}(\tau)<\infty
$$

Uma estimativa similar pode ser obtida para $H_{2,3}$ e $H_{2,4}$. Portanto, podemos encontrar uma constante $C_{2}(q, \tau)>0$ tal que $H_{2} \leq C_{2}(q, \tau)$.

A estimativa de $H_{3}$ é obtida da seguinte forma: como $r>3 / 2$, então $\left|r e^{i \theta}-1\right| \geq|r-1|=$ $r-1 \geq r / 3 \mathrm{e}$

$$
\begin{aligned}
H_{3} & \leq 3^{q} \int_{0}^{2 \pi} \int_{\frac{3}{2}}^{R} \frac{d r d \theta}{|\sin (\theta+\varphi)|^{\tau} r^{2 q+\tau-1}} \\
& \leq 3^{q} C_{3}(\tau) \int_{\frac{3}{2}}^{R} \frac{d r}{r^{2 q+\tau-1}} \leq \frac{3^{q} C_{3}(\tau)}{2 q+\tau-2}\left(\frac{2}{3}\right)^{2 q+\tau-2},
\end{aligned}
$$

onde $C_{3}(\tau)$ é uma constante positiva. Assim, completamos a prova de (4.4) no caso $\gamma=0$.

Caso 2: $\gamma>0$. Neste caso, $H$ (dado por (4.4)) pode ser reescrito da forma $H=H_{1}+H_{2}$, onde

$$
\begin{aligned}
& H_{1}=\int_{0}^{2 \pi} \int_{0}^{\gamma} \frac{r d r d \theta}{|\gamma+r \sin (\theta+\varphi)|^{\tau} r^{q}\left|r e^{i \theta}-1\right|^{q}}, \\
& H_{2}=\int_{0}^{2 \pi} \int_{\gamma}^{R} \frac{r d r d \theta}{|\gamma+r \sin (\theta+\varphi)|^{\tau} r^{q}\left|r e^{i \theta}-1\right|^{q}} .
\end{aligned}
$$


Para estimar $H_{1}$, consideramos três casos que dependem dos valores de $\gamma$.

Primeiro caso: $0<\gamma<\frac{1}{2}$. Neste caso, como $0<r<1 / 2$, temos $\left|r e^{i \theta}-1\right| \geq 1-r \geq 1 / 2$. Assim, pelo Lema 6 (página 62),

$$
H_{1} \leq 2^{q} \int_{0}^{2 \pi} \int_{0}^{\gamma} \frac{d r d \theta}{|\gamma+r \sin (\theta+\varphi)|^{\tau} r^{q-1}} \leq 2^{q} M(q, \tau) .
$$

Segundo caso: $\frac{1}{2}<\gamma<\frac{3}{2}$. Neste caso escrevemos $H_{1}=H_{1,1}+H_{1,2}$, onde

$$
\begin{aligned}
& H_{1,1}=\int_{0}^{2 \pi} \int_{0}^{\frac{1}{2}} \frac{r d r d \theta}{|\gamma+r \sin (\theta+\varphi)|^{\tau} r^{q}\left|r e^{i \theta}-1\right|^{q}} \\
& H_{1,2}=\int_{0}^{2 \pi} \int_{\frac{1}{2}}^{\gamma} \frac{r d r d \theta}{|\gamma+r \sin (\theta+\varphi)|^{\tau} r^{q}\left|r e^{i \theta}-1\right|^{q}} .
\end{aligned}
$$

Note que a estimativa de $H_{1,1}$ é dada pelo caso anterior.

Para $H_{1,2}$ temos

$$
\begin{aligned}
H_{1,2} & \leq 2^{q-1} \int_{0}^{2 \pi} \int_{\frac{1}{2}}^{\gamma} \frac{d r d \theta}{|\gamma-r|^{\tau}\left|r e^{i \theta}-1\right|^{q}} \\
& =2^{q-1} \sum_{j=1}^{4} \int_{\frac{(j-1) \pi}{2}}^{\frac{j \pi}{2}} \int_{\frac{1}{2}}^{\gamma} \frac{d r d \theta}{|\gamma-r|^{\tau}\left|r e^{i \theta}-1\right|^{q}}=2^{q-1} \sum_{j=1}^{4} H_{1,2}^{j} .
\end{aligned}
$$

Para estimar $H_{1,2}^{1}$ escrevemos

$$
H_{1,2}^{1}=\int_{0}^{\theta_{0}} \int_{\frac{1}{2}}^{\gamma} \frac{d r d \theta}{|\gamma-r|^{\tau}\left|r e^{i \theta}-1\right|^{q}}+\int_{\theta_{0}}^{\frac{\pi}{2}} \int_{\frac{1}{2}}^{\gamma} \frac{d r d \theta}{|\gamma-r|^{\tau}\left|r e^{i \theta}-1\right|^{q}}
$$

com $0<\theta_{0}<\pi / 2$ tal que $1-\theta^{2} / 2<\cos \theta<1-\theta^{2} / 4$ para $0<\theta<\theta_{0}$. Note que essa escolha de $\theta_{0}$ implica que $\left|r e^{i \theta}-1\right|>1-\cos \theta_{0}$ para $\theta \geq \theta_{0}$. Assim,

$$
H_{1,2}^{1} \leq 2^{q} \int_{0}^{\theta_{0}} \int_{\frac{1}{2}}^{\gamma} \frac{d r d \theta}{|\gamma-r|^{\tau}\left((r-1)^{2}+\theta^{2}\right)^{\frac{q}{2}}}+\frac{\frac{\pi}{2}-\theta_{0}}{\left(1-\cos \theta_{0}\right)^{\frac{q}{2}}} \frac{1}{1-\tau}
$$

Agora, pelo uso das coordenadas polares $r=1+\rho \sin \phi, \theta=\rho \cos \phi$ na integral, e usando o Lema 6 (página 62), obtemos

$$
\int_{0}^{\theta_{0}} \int_{\frac{1}{2}}^{\gamma} \frac{d r d \theta}{|\gamma-r|^{\tau}\left((r-1)^{2}+\theta^{2}\right)^{\frac{q}{2}}} \leq \int_{0}^{1} \int_{0}^{2 \pi} \frac{d \varphi d \rho}{|\gamma-1-\rho \sin \varphi|^{\tau} \rho^{q-1}} \leq M(q, \tau) .
$$

Assim, podemos encontrar uma constante $C_{1}(q, \tau)>0$ tal que $H_{1,2}^{1} \leq C_{1}(q, \tau)$. Procedendo como acima, podemos encontrar uma constante $C_{2}(q, \tau)>0$ tal que $H_{1,2}^{4} \leq C_{2}(q, \tau)$.

Para $H_{1,2}^{2}$, temos

$$
H_{1,2}^{2}=\int_{\frac{3 \pi}{2}}^{2 \pi} \int_{\frac{1}{2}}^{\gamma} \frac{d r d \theta}{|\gamma-r|^{\tau}\left|r e^{i \theta}+1\right|^{q}} \leq \int_{\frac{3 \pi}{2}}^{2 \pi} \int_{\frac{1}{2}}^{\gamma} \frac{d r d \theta}{|\gamma-r|^{\tau}} \leq \frac{\pi}{2(1-\tau)},
$$


uma vez que $\left|r e^{i \theta}+1\right| \geq 1$ para $\frac{3 \pi}{2} \leq \theta \leq 2 \pi$. Uma estimativa análoga pode ser usada para mostrar que $H_{1,2}^{3} \leq \frac{\pi}{2(1-\tau)}$. Portanto, podemos encontrar uma constante $C_{3}(q, \tau)>0$ tal que $H_{1} \leq C_{3}(q, \tau)$.

Terceiro caso: $\gamma>\frac{3}{2}$. Neste caso, escrevemos $H_{1}=H_{1,1}+H_{1,2}$, onde

$$
\begin{aligned}
& H_{1,1}=\int_{0}^{2 \pi} \int_{0}^{\frac{3}{2}} \frac{r d r d \theta}{|\gamma+r \sin (\theta+\varphi)|^{\tau} r^{q}\left|r e^{i \theta}-1\right|^{q}} \\
& H_{1,2}=\int_{0}^{2 \pi} \int_{\frac{3}{2}}^{\gamma} \frac{r d r d \theta}{|\gamma+r \sin (\theta+\varphi)|^{\tau} r^{q}\left|r e^{i \theta}-1\right|^{q}}
\end{aligned}
$$

A estimativa para $H_{1,1}$ segue do caso anterior.

Para $H_{1,2}$, note que, como $r \geq 3 / 2$ temos $\left|r e^{i \theta}-1\right| \geq r / 2 \mathrm{e}$

$$
H_{1,2} \leq 2^{q} \int_{0}^{2 \pi} \int_{\frac{3}{2}}^{\gamma} \frac{d r d \theta}{|\gamma+r \sin (\theta+\varphi)|^{\tau} r^{2 q-1}}=C(q, \tau)<\infty .
$$

Agora vamos estimar $\mathrm{H}_{2}$. Lembre-se que

$$
H_{2}=\int_{0}^{2 \pi} \int_{\gamma}^{R} \frac{r d r d \theta}{|\gamma+r \sin (\theta+\varphi)|^{\tau} r^{q}\left|r e^{i \theta}-1\right|^{q}}
$$

Como feito para $H_{1}$, vamos considerar três casos:

Primeiro caso: $\frac{3}{2}<\gamma<R$. Como $r>\frac{3}{2}$ implica $\left|r e^{i \theta}-1\right| \geq \frac{r}{3}$, temos

$$
H_{2} \leq 3^{q} \int_{0}^{2 \pi} \int_{\gamma}^{R} \frac{d r d \theta}{|\gamma+r \sin (\theta+\varphi)|^{\tau} r^{2 q-1}}=3^{q} \int_{0}^{2 \pi} \int_{\gamma}^{R} \frac{d r d \theta}{|\gamma+r \sin \theta|^{\tau} r^{2 q-1}}
$$

Assim, a estimativa para $\mathrm{H}_{2}$ segue do Lema 5 (página 62).

Segundo caso: $\frac{1}{2}<\gamma<\frac{3}{2}$. Neste caso vamos escrever $H_{2}=H_{2,1}+H_{2,2}$, onde

$$
\begin{aligned}
& H_{2,1}=\int_{-\frac{\pi}{2}}^{\frac{3 \pi}{2}} \int_{\gamma}^{\frac{3}{2}} \frac{r d r d \theta}{|\gamma+r \sin (\theta+\varphi)|^{\tau} r^{q}\left|r e^{i \theta}-1\right|^{q}}=H_{2,1}^{1}+H_{2,1}^{2} \\
& H_{2,2}=\int_{0}^{2 \pi} \int_{\frac{3}{2}}^{R} \frac{r d r d \theta}{|\gamma+r \sin (\theta+\varphi)|^{\tau} r^{q}\left|r e^{i \theta}-1\right|^{q}}
\end{aligned}
$$

e,

$$
\begin{aligned}
& H_{2,1}^{1}=\int_{-\frac{\pi}{2}}^{\frac{\pi}{2}} \int_{\gamma}^{\frac{3}{2}} \frac{d r d \theta}{|\gamma+r \sin \theta|^{\tau} r^{q-1}\left|r e^{i(\theta-\varphi)}-1\right|^{q}} \\
& H_{2,1}^{2}=\int_{\frac{\pi}{2}}^{\frac{3 \pi}{2}} \int_{\gamma}^{\frac{3}{2}} \frac{d r d \theta}{|\gamma+r \sin \theta|^{\tau} r^{q-1}\left|r e^{i(\theta-\varphi)}-1\right|^{q}}
\end{aligned}
$$


Note que a estimativa de $H_{2,2}$ é dada pelo caso anterior.

Para estimar $H_{2,1}^{1}$, note que, como $1 / 2<\gamma<r<3 / 2$ temos $1 / 3<\gamma / r<1$. Seja $\theta_{1} \in$ $\left(-\pi / 2,-\alpha_{0}\right)$ tal que $-\gamma / r=\sin \theta_{1}$, onde $\sin \alpha_{0}=1 / 3$. Temos então,

$$
\frac{1}{|\gamma+r \sin \theta|^{\tau}}=\frac{\left|\theta-\theta_{1}\right|^{\tau}}{r^{\tau}\left|\sin \theta-\sin \theta_{1}\right|^{\tau}} \frac{1}{\left|\theta-\theta_{1}\right|^{\tau}} \leq \frac{A(\tau)}{r^{\tau}\left|\cos \theta_{1}\right|^{\tau}} \frac{1}{\left|\theta-\theta_{1}\right|^{\tau}},
$$

para alguma constante $A(\tau)>0$; assim, substituindo $\cos \theta_{1}$ por $\sqrt{r^{2}-\gamma^{2}} / r$ obtemos

$$
\frac{1}{|\gamma+r \sin \theta|^{\tau}} \leq \frac{A(\tau)}{\left(r^{2}-\gamma^{2}\right)^{\frac{\tau}{2}}} \frac{1}{\left|\theta-\theta_{1}(r)\right|^{\tau}}
$$

Portanto,

$$
\begin{aligned}
H_{2,1}^{1} & \leq 2^{q-1} A(\tau) \int_{-\frac{\pi}{2}}^{\frac{\pi}{2}} \int_{\gamma}^{\frac{3}{2}} \frac{d r d \theta}{\left(r^{2}-\gamma^{2}\right)^{\frac{\tau}{2}}\left|\theta-\theta_{1}(r)\right| \tau\left|r e^{i(\theta-\varphi)}-1\right|^{q}} \\
& =2^{q-1} A(\tau) \int_{-\frac{\pi}{2}-\varphi}^{\frac{\pi}{2}-\varphi} \int_{\gamma}^{\frac{3}{2}} \frac{d r d \theta}{\left(r^{2}-\gamma^{2}\right)^{\frac{\tau}{2}}\left|\theta-\theta_{1}(r)+\varphi\right|^{\tau}\left|r e^{i \theta}-1\right|^{q}} \\
& \leq 2^{q-1} A(\tau) \sum_{j=1}^{4} \int_{\frac{(j-4) \pi}{2}}^{\frac{(j-3) \pi}{2}} \int_{\gamma}^{\frac{3}{2}} \frac{d r d \theta}{\left(r^{2}-\gamma^{2}\right)^{\frac{\tau}{2}}\left|\theta-\theta_{1}(r)+\varphi\right|^{\tau}\left|r e^{i \theta}-1\right|^{q}} \\
& =2^{q-1} A(\tau) \sum_{j=1}^{4} H_{2,1}^{1, j} .
\end{aligned}
$$

Temos

$$
H_{2,1}^{1,1}=\int_{-\frac{\pi}{2}}^{0} \int_{\gamma}^{\frac{3}{2}} \frac{d r d \theta}{\left(r^{2}-\gamma^{2}\right)^{\frac{\tau}{2}}\left|\theta-\pi-\theta_{1}(r)+\varphi\right|^{\tau}\left|r e^{i \theta}+1\right|^{q}} .
$$

Para $-\pi / 2 \leq \theta \leq 0$ temos $\left|r e^{i \theta}+1\right| \geq 1+r \cos \theta \geq 1 \mathrm{e}$

$$
H_{2,1}^{1,1} \leq \int_{-\frac{\pi}{2}}^{0} \int_{\gamma}^{\frac{3}{2}} \frac{d r d \theta}{\left(r^{2}-\gamma^{2}\right)^{\frac{\tau}{2}}\left|\theta-\pi-\theta_{1}(r)+\varphi\right|^{\tau}} \leq C(\tau)
$$

para uma constante $C(\tau)>0$. De forma semelhante, $H_{2,1}^{1,2} \leq \tilde{C}(\tau)$, para alguma constante $\tilde{C}(\tau)>$ 0 .

Para estimar $H_{2,1}^{1,3}$, seja $0<\theta_{0}<\alpha_{0} / 2$ tal que

$$
1-\frac{\theta^{2}}{2}<\cos \theta<1-\frac{\theta^{2}}{4}, \quad \text { para } \quad-\theta_{0} \leq \theta \leq 0 .
$$

Note que $-\theta_{0} \leq \theta \leq 0$ implica

$$
\theta-\theta_{1}(r)+\varphi \geq-\theta_{0}-\theta_{1}(r)+\varphi \geq \alpha_{0}-\frac{\alpha_{0}}{2}+\varphi \geq \frac{\alpha_{0}}{2} .
$$

Além disso, para $-\pi / 2 \leq \theta \leq-\theta_{0}$ temos

$$
\left|r e^{i \theta}-1\right|^{2} \geq 1-\cos \theta_{0}
$$


Assim,

$$
\begin{aligned}
H_{2,1}^{1,3} & \leq\left(\frac{2}{\alpha_{0}}\right)^{\tau} \int_{-\theta_{0}}^{0} \int_{\gamma}^{\frac{3}{2}} \frac{d r d \theta}{\left(r^{2}-\gamma^{2}\right)^{\frac{\tau}{2}}\left|r e^{i \theta}-1\right|^{q}} \\
& +\left(\frac{1}{1-\cos \theta_{0}}\right)^{\frac{q}{2}} \int_{-\frac{\pi}{2}}^{-\theta_{0}} \int_{\gamma}^{\frac{3}{2}} \frac{d r d \theta}{\left(r^{2}-\gamma^{2}\right)^{\frac{\tau}{2}}\left|\theta-\theta_{1}(r)+\varphi\right|^{\tau}} \\
& \leq\left(\frac{2}{\alpha_{0}}\right)^{\tau} \int_{-\theta_{0}}^{0} \int_{\gamma}^{\frac{3}{2}} \frac{d r d \theta}{\left(r^{2}-\gamma^{2}\right)^{\frac{\tau}{2}}\left|r e^{i \theta}-1\right|^{q}}+C_{1}(\tau, q),
\end{aligned}
$$

para uma constante $C_{1}(\tau, q)>0$. Note que, para $r>1 / 2$ e $-\theta_{0} \leq \theta \leq 0$ temos

$$
\left|r e^{i \theta}-1\right|^{2} \geq \frac{(r-1)^{2}+\theta^{2}}{4}
$$

assim, como $r \geq \gamma \geq 1 / 2$ implica $r^{2}-\gamma^{2} \geq r-\gamma$, temos

$$
\begin{aligned}
\int_{-\theta_{0}}^{0} \int_{\gamma}^{\frac{3}{2}} \frac{d r d \theta}{\left(r^{2}-\gamma^{2}\right)^{\frac{\tau}{2}}\left|r e^{i \theta}-1\right|^{q}} & \leq 2^{q} \int_{-\theta_{0}}^{0} \int_{\gamma}^{\frac{3}{2}} \frac{d r d \theta}{(r-\gamma)^{\frac{\tau}{2}}\left((r-1)^{2}+\theta^{2}\right)^{\frac{q}{2}}} \\
& \leq 2^{q} \int_{D((0,1) ; 1)} \frac{d r d \theta}{(r-\gamma)^{\frac{\tau}{2}}\left((r-1)^{2}+\theta^{2}\right)^{\frac{q}{2}}} \\
& =2^{q} \int_{0}^{1} \int_{0}^{2 \pi} \frac{d r d \theta}{|1-\gamma+\rho \sin \alpha|^{\frac{\tau}{2}} \rho^{q-1}} \leq C_{2}(\tau, q),
\end{aligned}
$$

onde a última estimativa segue do Lema 6 (página 62). Portanto, podemos encontrar uma constante $C_{3}(\tau, q)>0$ tal que $H_{2,1}^{1,3} \leq C_{3}(\tau, q)$. Podemos usar argumentos similares para estimar $H_{2,1}^{1,4}$ e $H_{2,1}^{2}$. Portanto, podemos encontrar uma constante $C(\tau, q)>0$ para qual $H_{2,1} \leq C(\tau, q)$.

Terceiro caso: $0<\gamma<\frac{1}{2}$. Neste caso, vamos escrever a integral $\mathrm{H}_{2}$ da seguinte forma

$$
H_{2}=\int_{0}^{2 \pi} \int_{\gamma}^{\frac{1}{2}} \frac{r d r d \theta}{|\gamma+r \sin (\theta+\varphi)|^{\tau} r^{q}\left|r e^{i \theta}-1\right|^{q}}+\int_{0}^{2 \pi} \int_{\frac{1}{2}}^{R} \frac{r d r d \theta}{|\gamma+r \sin (\theta+\varphi)|^{\tau} r^{q}\left|r e^{i \theta}-1\right|^{q}} .
$$

A segunda integral pode ser estimada como feito anteriormente. Assim, é suficiente estimar a primeira integral. Como $r<1 / 2$ implica $\left|r e^{i \theta}-1\right| \geq|r-1| \geq 1 / 2$, temos

$$
\int_{0}^{2 \pi} \int_{\gamma}^{\frac{1}{2}} \frac{r d r d \theta}{|\gamma+r \sin (\theta+\varphi)|^{\tau} r^{q}\left|r e^{i \theta}-1\right|^{q}} \leq 2^{q} \int_{0}^{2 \pi} \int_{0}^{\frac{1}{2}} \frac{d r d \theta}{|\gamma+r \sin (\theta+\varphi)|^{\tau} r^{q-1}}
$$

que pode ser estimada aplicando o Lema 6 (página 62).

Isto completa a prova do caso $\gamma>0$ e assim concluímos a demonstração do lema. 
CAPÍTULO

\section{5}

10

A EQUAÇÃO $L u=f$

Neste capítulo vamos tratar da equação $L u=f, \operatorname{com} f$ em um espaço $L^{p}$ adequado. Com a técnica desenvolvida, será possível estudar equações da forma $L u=F(x, y, u)$; em particular, estudaremos também a equação $L u=a u+b \bar{u}, \operatorname{com} a, b \in L^{p}$.

\subsection{O operador integral $T_{Z}$}

Seja $L$ uma campo vetorial complexo, no aberto $\tilde{\Omega}$ de $\mathbb{R}^{2}$, pertencente a classe $\mathscr{X}(\tilde{\Omega})$. Isto é, (como definido no Capítulo 3, página 47) o campo $L$ satisfaz as seguintes condições:

(i) $L$ é um campo não singular de classe $C^{\varepsilon}, 0<\varepsilon<1$, em $\tilde{\Omega}$;

(ii) O conjunto característico $\Sigma$ de $L$ é uma subvariedade mergulhada de dimensão 1 de $\tilde{\Omega}$, de classe $C^{1+\varepsilon}$, com um número finito de componentes conexas;

(iii) $L$ é de classe $C^{1}$ em $\tilde{\Omega} \backslash \Sigma$;

(iv) Para cada $\mathrm{p} \in \tilde{\Omega} \backslash \Sigma$, existe uma integral primeira $Z: U \rightarrow \mathbb{C}$ de $L$, de classe $C^{2}$, com $U \subset \tilde{\Omega} \backslash \Sigma$ aberto e $p \in U$, tal que $Z: U \rightarrow Z(U) \subset \mathbb{C}$ é um homeomorfismo;

(v) Para cada $\mathrm{p} \in \Sigma$, existem uma integral primeira $Z: U \rightarrow \mathbb{C}$ de $L$, com $U \subset \tilde{\Omega}$ uma vizinhança aberta de p, tal que $Z: U \rightarrow Z(U) \subset \mathbb{C}$ é um homeomorfismo que satisfaz $Z_{x y}, Z_{y x} \in C(U)$ e $Z_{x y}=Z_{y x}$ em $U$, e coordenadas locais $(s, t)$ em $U$, de classe $C^{2}$ centradas em $\mathrm{p}$, tal que nas novas variáveis a integral primeira $Z$ se escreve como

$$
Z_{\sigma, \beta}(s, t)=s+i\left(\frac{t|t|^{\sigma}}{\sigma+1}+\beta(s)\right)
$$

onde $\beta$ é uma função a valores reais de classe $C^{2+\varepsilon}$ que depende apenas de $s$ e $\sigma \geq \varepsilon$ é uma constante. Além disso, $Z$ satisfaz a seguinte condição: se $L=A \partial_{x}+B \partial_{y}$ em $\tilde{\Omega}$, então 
a função $\lambda: U \rightarrow \mathbb{C}$ definida por

$$
\lambda(x, y)= \begin{cases}\frac{A(x, y)}{Z_{y}(x, y)}, & \text { se } Z_{y}(x, y) \neq 0 \\ -\frac{B(x, y)}{Z_{x}(x, y)}, & \text { se } Z_{x}(x, y) \neq 0\end{cases}
$$

é de classe $C^{1}$ em $U$.

Seja

$$
Z: \tilde{\Omega} \rightarrow Z(\tilde{\Omega}) \subset \mathbb{C}
$$

uma integral primeira global de $L$, de classe $C^{1+\varepsilon}$, que é um homeomorfismo sobre sua imagem (veja a Proposição 7, página 37).

Defina

$$
\sigma_{\mathrm{M}}=\max _{1 \leq j \leq N}\left\{\sigma_{j}\right\}
$$

onde $\sigma_{1}, \cdots, \sigma_{N}$ são os respectivos tipos de $L$ ao longo das componentes conexas de $\Sigma$, a saber, $\Sigma_{1}, \cdots, \Sigma_{N}$. (Veja a Proposição 10, página 51, e a Observação 21, página 52). Também definimos

$$
\tau_{\mathrm{M}}=\frac{\sigma_{\mathrm{M}}}{\sigma_{\mathrm{M}}+1}
$$

Note que $0<\tau_{\mathrm{M}}<1$

Seja $\Omega$ um aberto compactamente contido em $\tilde{\Omega}$, isto é, $\bar{\Omega}$ é um compacto de $\mathbb{R}^{2}$ e $\bar{\Omega} \subset \tilde{\Omega}$.

Para $f \in L^{1}(\Omega)$, definimos o operador integral

$$
T_{Z} f(x, y)=\frac{1}{2 \pi i} \int_{\Omega} \frac{f(\xi, \eta)}{Z(\xi, \eta)-Z(x, y)} d \xi d \eta,(x, y) \in \tilde{\Omega} .
$$

Veja em (B.2), página 136, o operador integral clássico $T f$ que foi inspiração para a definição do operador integral acima.

Observação 22. Operadores integrais, como $T_{Z} f$, foram usados em (MEZIANI, 2008) e (MEZIANI, 2012) em conexão com outras classes de campos vetoriais.

Temos o seguinte teorema:

Teorema 4. Seja $\sigma_{\mathrm{M}}$ dado por (5.4) e seja $f \in L^{p}(\Omega)$, com $p>2+\sigma_{\mathrm{M}}$. Então, existe uma constante $M=M\left(p, \sigma_{1}, \ldots, \sigma_{N}, \Omega\right)>0$ tal que o operador $T_{Z}$ satisfaz

$$
\left|T_{Z} f(x, y)\right| \leq \frac{1}{2 \pi} \int_{\Omega} \frac{|f(\xi, \eta)|}{|Z(\xi, \eta)-Z(x, y)|} d \xi d \eta \leq M\|f\|_{p}, \quad \forall(x, y) \in \bar{\Omega} .
$$


Demonstração. Seja $(x, y) \in \Omega$. Então, pela desigualdade de Hölder, temos

$$
\left|T_{Z} f(x, y)\right| \leq \frac{1}{2 \pi} \int_{\Omega} \frac{|f(\xi, \eta)|}{|Z(\xi, \eta)-Z(x, y)|} d \xi d \eta \leq \frac{\|f\|_{p}}{2 \pi}\left(\int_{\Omega} \frac{d \xi d \eta}{|Z(\xi, \eta)-Z(x, y)|^{q}}\right)^{\frac{1}{q}}
$$

onde $q=p /(p-1)$.

Seja $\left\{V_{k}\right\}_{k=1, \ldots, k_{0}}$ uma cobertura finita do compacto $\Sigma \cap \bar{\Omega}$, formada por abertos pré compactos de $\tilde{\Omega}$, tais que cada aberto $V_{k}$ intercepta apenas uma componente conexa de $\Sigma$ e para cada $k$, com $V_{k} \cap \Sigma_{j} \neq \emptyset$, podemos escrever

$$
\left.Z\right|_{V_{k}}=G_{k} \circ Z_{\sigma_{j}} \circ F_{k} \text { em } V_{k}
$$

onde $F_{k}: \overline{V_{k}} \rightarrow \overline{U_{k}}, G_{k}: \overline{Z_{\sigma_{j}}\left(U_{k}\right)} \rightarrow \overline{B_{k}}$ são difeomorfismos de classe $C^{1} \mathrm{e}$

$$
Z_{\sigma_{j}}(s, t)=s+i \frac{t|t|^{\sigma_{j}}}{1+\sigma_{j}},(s, t) \in \mathbb{R}^{2}
$$

(Veja o Corolário 2, página 56). Como $\Sigma$ é uma subvariedade mergulhada de $\Omega$ com dimensão 1 e $\bar{\Omega}$ é compacto, existe uma família de abertos de $\tilde{\Omega},\left\{V_{k}\right\}_{k=k_{0}+1, \ldots, m}$, com $\overline{V_{k}}$ compacto, $\overline{V_{k}} \cap \Sigma=\emptyset$, e tal que

$$
\bar{\Omega} \subset V_{1} \cup \ldots \cup V_{k_{0}} \cup V_{k_{0}+1} \cup \ldots \cup V_{m},
$$

isto é, $\mathscr{C}=\left\{V_{k}\right\}_{k=1, \ldots, m}$ é uma cobertura de $\bar{\Omega}$. Seja $\left\{\varphi_{k}\right\}_{k=1, \ldots, m}$ uma partição da unidade subordinada a cobertura $\mathscr{C}$. Assim, temos

$$
\begin{gathered}
\int_{\Omega} \frac{d \xi d \eta}{|Z(\xi, \eta)-Z(x, y)|^{q}}=\int_{\Omega} \frac{\sum_{k=1}^{m} \varphi_{k}(\xi, \eta) d \xi d \eta}{|Z(\xi, \eta)-Z(x, y)|^{q}} \\
=\sum_{k=1}^{m} \int_{V_{k} \cap \Omega} \frac{\varphi_{k}(\xi, \eta) d \xi d \eta}{|Z(\xi, \eta)-Z(x, y)|^{q}}=: \sum_{k=1}^{m} I_{k}(x, y) .
\end{gathered}
$$

$\operatorname{Vamos} \operatorname{estimar} I_{k}(x, y)$ para $(x, y) \in \tilde{\Omega}$ e $k \in\{1, \ldots, m\}$.

Caso 1: $(x, y) \in V_{k}$ e $k \in\left\{1, \ldots, k_{0}\right\}$.

Seja $j \in\{1, \ldots, N\}$ tal que $V_{k} \cap \Sigma_{j} \neq 0$. Temos,

$$
\begin{aligned}
& \int_{V_{k}} \frac{d \xi d \eta}{|Z(\xi, \eta)-Z(x, y)|^{q}}=\int_{V_{k}} \frac{d \xi d \eta}{\mid G_{k}\left(Z_{\sigma_{j}}\left(F_{k}(\xi, \eta)\right)\right)-G_{k}\left(\left.Z_{\sigma_{j}}\left(F_{k}(x, y)\right)\right|^{q}\right.} \\
\leq & K \int_{V_{k}} \frac{d \xi d \eta}{\left|Z_{\sigma_{j}}\left(F_{k}(\xi, \eta)\right)-Z_{\sigma_{j}}\left(F_{k}(x, y)\right)\right|^{q}} \leq K N \int_{F_{k}\left(V_{k}\right)} \frac{d s d t}{\left|Z_{\sigma_{j}}(s, t)-Z_{\sigma_{j}}\left(s^{\circ}, t^{\circ}\right)\right|^{q}},
\end{aligned}
$$

onde, $\left(s^{\circ}, t^{\circ}\right)=F_{k}(x, y), K$ é uma constante de Lipschitz para toda $G_{\ell}^{-1}$, isto é,

$$
\left|\mathrm{q}_{1}-\mathrm{q}_{2}\right| \leq K\left|G_{\ell}\left(\mathrm{q}_{1}\right)-G_{\ell}\left(\mathrm{q}_{2}\right)\right|, \mathrm{q}_{1}, \mathrm{q}_{2} \in \overline{B_{\ell}}
$$

para todo $\ell \in\left\{1, \ldots, k_{0}\right\}$, e $N \geq \sup \left\{\left|\operatorname{det}\left(D F_{\ell}\right)(\xi, \eta)\right|^{-1} ;(\xi, \eta) \in V_{\ell}\right\}$, para todo $\ell \in\left\{1, \ldots, k_{0}\right\}$. 
Usando a mudança de variáveis $\xi=s$ e $\eta=\frac{t|t|^{\sigma_{j}}}{1+\sigma_{j}}$ obtemos

$$
\begin{aligned}
\left(1+\sigma_{j}\right)^{\tau_{j}} \int_{U_{k}} \frac{d s d t}{\left|Z_{\sigma_{j}}(s, t)-Z_{\sigma_{j}}\left(s^{\circ}, t^{\circ}\right)\right|^{q}} & =\int_{Z_{\sigma_{j}}\left(U_{k}\right)} \frac{d \xi d \eta}{|\eta|^{\tau_{j}}|\zeta-z|^{q}} \\
& \leq \int_{D\left(z, \mathrm{~d}_{0}\right)} \frac{d \xi d \eta}{|\eta|^{\tau_{j}|\zeta-z|^{q}}}, \tau_{j}=\sigma_{j} /\left(\sigma_{j}+1\right)
\end{aligned}
$$

onde $\zeta=\xi+i \eta, z=Z_{\sigma_{j}}\left(s^{\circ}, t^{\circ}\right)$, e $\mathrm{d}_{0} \geq \operatorname{diam}\left(Z_{\sigma_{n}}\left(U_{\ell}\right)\right)$, para todo $\ell \in\left\{1, \ldots, k_{0}\right\}$ e para todo $n \in\{1, \ldots, N\}$ com $V_{\ell} \cap \Sigma_{n} \neq 0$. Agora, com o uso das coordenadas polares $(r, \theta)$ dadas por

$$
\xi=r \cos \theta+\mathfrak{R}(z) \text { e } \eta=r \sin \theta+\mathfrak{I}(z)
$$

e pelo Lema 6 (página 62) temos

$$
\int_{D\left(z, \mathrm{~d}_{0}\right)} \frac{d \xi d \eta}{|\eta|^{\tau_{j}}|\zeta-z|^{q}}=\int_{0}^{2 \pi} \int_{0}^{\mathrm{d}_{0}} \frac{d r d \theta}{|r \sin \theta+\mathfrak{I}(z)|^{\tau_{j} r^{q-1}}} \leq M\left(q, \tau_{j}\right) \mathrm{d}_{0}{ }^{2-\tau_{j}-q}
$$

pois $p>2+\sigma_{\mathrm{M}}$ implica $1<q<2-\tau_{\mathrm{M}} \leq 2-\tau_{n}$, para todo $n \in\{1, \ldots, N\}$ (lembrando que $\left.\tau_{\mathrm{M}}=\sigma_{\mathrm{M}} /\left(\sigma_{\mathrm{M}}+1\right)\right)$. Assim, existe uma constante $M_{0}=M_{0}\left(q, \sigma_{1}, \ldots, \sigma_{N}, \Omega\right)>0$ tal que

$$
I_{k}(x, y) \leq M_{0}
$$

Caso 2: $(x, y) \in V_{k}$ e $k \in\left\{k_{0}+1, \ldots, m\right\}$.

Temos

$$
I_{k}(x, y) \leq \int_{V_{k}} \frac{d \xi d \eta}{|Z(\xi, \eta)-Z(x, y)|^{q}} \leq C \int_{Z\left(V_{k}\right)} \frac{d \xi d \eta}{|\zeta-Z(x, y)|^{q}},
$$

onde $C \geq \sup \left\{|\operatorname{det}(D Z)(\xi, \eta)|^{-1} ;(\xi, \eta) \in V_{\ell}\right\}$, para todo $\ell \in\left\{k_{0}+1, \ldots, m\right\}$.

Seja $\mathrm{d}>0$ tal que $\mathrm{d} \geq \operatorname{diam}\left(Z\left(V_{\ell}\right)\right)=\sup \left\{\left|\zeta_{1}-\zeta_{2}\right| ; \zeta_{1}, \zeta_{2} \in Z\left(V_{\ell}\right)\right\}$, para todo $\ell \in$ $\left\{k_{0}+1, \ldots, m\right\}$. Temos,

$$
\int_{Z\left(V_{k}\right)} \frac{d \xi d \eta}{|\zeta-Z(x, y)|^{q}} \leq \int_{D(Z(x, y), \mathrm{d})} \frac{d \xi d \eta}{|\zeta-Z(x, y)|^{q}}=\int_{D(0, \mathrm{~d})} \frac{d \xi d \eta}{|\zeta|^{q}}=2 \pi \int_{0}^{\mathrm{d}} s^{1-q} d s=\frac{2 \pi}{2-q} \mathrm{~d}^{2-q} .
$$

Assim, $M_{1}=M_{1}\left(q, \sigma_{1}, \ldots, \sigma_{N}, \Omega\right):=2 \pi C \mathrm{~d}^{2-q} /(2-q)$ é tal que

$$
I_{k}(x, y) \leq M_{1} .
$$

Caso 3: $(x, y) \in V_{k}^{c}$ e $k \in\{1, \ldots, m\}$.

Como supp $\varphi_{\ell} \subset V_{\ell}$, para todo $\ell \in\{1, \ldots, m\}$, temos que existe $\mu>0$ tal que

$$
0<\mu \leq \inf \left\{|Z(\xi, \eta)-Z(x, y)| ;(\xi, \eta) \in \operatorname{supp} \varphi_{\ell},(x, y) \in V_{\ell}^{c}\right\},
$$

para todo $\ell \in\{1, \ldots, m\}$. Assim,

$$
I_{k}(x, y)=\int_{V_{k} \cap \Omega} \frac{\varphi_{k}(\xi, \eta) d \xi d \eta}{|Z(\xi, \eta)-Z(x, y)|^{q}} \leq \int_{\operatorname{supp} \varphi_{k} \cap \Omega} \frac{d \xi d \eta}{|Z(\xi, \eta)-Z(x, y)|^{q}} \leq \frac{\left|\operatorname{supp} \varphi_{k} \cap \Omega\right|}{\mu^{q}} .
$$


Daí temos, que $M_{2}=M_{2}\left(q, \sigma_{1}, \ldots, \sigma_{N}, \Omega\right):=|\Omega| / \mu^{q}$ é tal que

$$
I_{k}(x, y) \leq M_{2} .
$$

Isso conclui o caso 3.

Portanto, para todo $(x, y) \in \tilde{\Omega}$ e todo $k \in\{1, \ldots, m\}$ temos

$$
I_{k}(x, y) \leq \max \left\{M_{0}, M_{1}, M_{2}\right\}=: M_{3} .
$$

Logo, de (5.8), temos

$$
\int_{\Omega} \frac{d \xi d \eta}{|Z(\xi, \eta)-Z(x, y)|^{q}} \leq m M_{3}, \text { para todo }(x, y) \in \tilde{\Omega} .
$$

De (5.6) e (5.9) obtemos o resultado.

O próximo corolário segue da demonstração do Teorema 4 acima.

Corolário 3. Sejam $Z: \tilde{\Omega} \rightarrow \mathbb{C}$ e $\Omega$ como acima. Se $1 \leq q<2-\tau_{\mathrm{M}}$, então existe uma constante $M=M\left(q, \sigma_{1}, \ldots, \sigma_{N}, \Omega\right)>0$ tal que

$$
\int_{\Omega} \frac{d \xi d \eta}{|Z(\xi, \eta)-Z(x, y)|^{q}} \leq M, \text { para todo }(x, y) \in \tilde{\Omega} .
$$

A demonstração da proposição que vem a seguir é fortemente inspirada na do caso clássico.

Proposição 15. Seja $f \in L^{1}(\Omega)$. Então, $T_{Z} f \in L^{q}(\Omega)$, para todo $1 \leq q<2-\tau_{\mathrm{M}}$.

Demonstração. Seja $f \in L^{1}(\Omega)$ e seja $g \in L^{p}(\Omega) \operatorname{com} p>2+\sigma_{\mathrm{M}}$. Pelo Teorema 4 a função

$$
g_{1}(x, y)=\int_{\Omega}|g(\xi, \eta)| \frac{d \xi d \eta}{|Z(\xi, \eta)-Z(x, y)|}
$$

é limitada. Assim, $f g_{1} \in L^{1}(\Omega)$. Aplicando o Teorema de Fubini obtemos

$$
\begin{aligned}
\int_{\Omega}|f(x, y)| g_{1}(x, y) d x d y & =\int_{\Omega}|f(x, y)|\left(\int_{\Omega}|g(\xi, \eta)| \frac{d \xi d \eta}{|Z(\xi, \eta)-Z(x, y)|}\right) d x d y \\
& =\int_{\Omega}|g(\xi, \eta)|\left(\int_{\Omega}|f(x, y)| \frac{d x d y}{|Z(\xi, \eta)-Z(x, y)|}\right) d \xi d \eta \\
& =\int_{\Omega}|g(\xi, \eta)| f_{1}(\xi, \eta) d \xi d \eta
\end{aligned}
$$

onde

$$
f_{1}(\xi, \eta)=\int_{\Omega}|f(x, y)| \frac{d x d y}{|Z(x, y)-Z(\xi, \eta)|} .
$$

Assim $|g| f_{1} \in L^{1}(\Omega)$. Como $g \in L^{p}(\Omega)$ é arbitraria, segue da recíproca da desigualdade de Hölder (Teorema 25, página 133) que $f_{1} \in L^{q}(\Omega)$, para $q=p /(p-1)$. Note que $p>2+\sigma_{M}$ implica $1<q<2-\tau_{\mathrm{M}}$. Note também que $\left|T_{Z} f\right| \leq f_{1}$. Portanto, $T_{Z} f \in L^{q}(\Omega)$, para qualquer $1<q<2-\tau_{\mathrm{M}}$. Como $\Omega$ é limitado, segue da desigualdade de Hölder que $T_{Z} f \in L^{1}(\Omega)$. 


\subsection{Existência de solução para a equação $L u=f$}

Sejam $L, Z, \tilde{\Omega}$ e $\Omega$ como na seção anterior. O próximo passo é mostrar que $T_{Z} f$ é solução da equação $H_{Z} u=f$ em $\Omega$, onde $H_{Z}$ é o Hamiltoniano de $Z$. A partir daí, obteremos uma solução para a equação $L u=f$ em $\Omega$. Para isso, vamos estudar uma fórmula de representação de soluções. Esta fórmula também será importante em outras partes deste texto.

Lema 8. (Teorema de Green) Seja $U$ um aberto conexo tal que $\bar{U}$ é compacto, $\bar{U} \subset \tilde{\Omega}$ e $\partial U$ é de classe $C^{1}$ por partes. Se $f, g \in C^{1}(\tilde{\Omega})$, então

$$
\int_{U}\left\{\partial_{x} f(x, y)-\partial_{y} g(x, y)\right\} d x d y=\int_{\partial U} g(x, y) d x+f(x, y) d y
$$

Essa versão do Teorema de Green para funções a valores complexos segue do caso real. Veja em (LINS-NETO, 2005) e (LIMA, 2008).

Seja $Z$ como em (5.3). Considere $H_{Z}$ como Halmitoniano de $Z$ em $\Omega$, isto é,

$$
H_{Z}=Z_{y} \frac{\partial}{\partial x}-Z_{x} \frac{\partial}{\partial y} \text { em } \tilde{\Omega}
$$

A próxima proposição é consequência do lema acima.

Proposição 16. Seja $U$ um aberto como no Lema 8. Se $w \in C^{1}(\tilde{\Omega})$. Então

$$
\int_{U} H_{Z} w d x d y=\int_{\partial U} w d Z(x, y) .
$$

Demonstração. Pelo Lema 8,

$$
\begin{gathered}
\int_{\partial U} w d Z=\int_{\partial U} w\left\{Z_{x} d x+Z_{y} d y\right\}=\int_{\partial U}\left(Z_{x} w\right) d x+\left(Z_{y} w\right) d y \\
=\int_{U}\left\{\left(Z_{y} w\right)_{x}-\left(Z_{x} w\right)_{y}\right\} d x d y=\int_{U}\left\{Z_{y x}-Z_{x y}\right\} w d x d y+\int_{U}\left\{Z_{y} w_{x}-Z_{x} w_{y}\right\} d x d y \\
=\int_{U}\left\{Z_{y x}-Z_{x y}\right\} w d x d y+\int_{U} H_{Z} w d x d y .
\end{gathered}
$$

Pela Proposição 9, página 49, $Z$ satisfaz $Z_{x y}=Z_{y x}$ em $U$. Logo,

$$
\int_{U}\left\{Z_{y x}-Z_{x y}\right\} w d x d y=0
$$

e por (5.12) temos o resultado.

O próximo lema é uma pequena adaptação de um teorema em (LINS-NETO, 2005). 
Lema 9. (Mudança de variáveis) Seja $U$ um aberto de $\mathbb{R}^{2}$ e sejam $w \in C(U)$ e $Z \in C^{1}(U)$ tal que $Z: U \rightarrow Z(U)$ é um homeomorfismo. Se $\gamma:[0,1] \rightarrow U$ uma curva de classe $C^{1}$ por partes em $U$, tal que $Z \circ \gamma$ é uma curva $C^{1}$ por partes em $Z(U)$, então

$$
\int_{\gamma} w(x, y) d Z(x, y)=\int_{Z \circ \gamma}\left(w \circ Z^{-1}\right)(\zeta) d \zeta, \zeta=\xi+i \eta
$$

Demonstração. Considere $\gamma(t)=\left(\gamma_{1}(t), \gamma_{2}(t)\right), Z_{1}=\Re(Z), Z_{2}=\mathfrak{I}(Z)$ e $\tilde{w}(\zeta)=\left(w \circ Z^{-1}\right)(\zeta)$. Vamos demonstrar a fórmula (5.13) no caso em que $\gamma$ é de classe $C^{1}$. O caso geral segue dividindo os intervalos de integração convenientemente.

Pela definição de integral sobre curvas e pela regra da cadeia temos

$$
\begin{aligned}
& \int_{\gamma} w(x, y) d Z(x, y)=\int_{0}^{1} w(\gamma(t))\left\{Z_{x}(\gamma(t)) \gamma_{1}^{\prime}(t)+Z_{y}(\gamma(t)) \gamma_{2}^{\prime}(t)\right\} d t \\
& =\int_{0}^{1} \tilde{w}(Z(\gamma(t)))\left\{\left[Z_{1 x}(\gamma(t))+i Z_{2 x}(\gamma(t))\right] \gamma_{1}^{\prime}(t)+\left[Z_{1 y}(\gamma(t))+i Z_{2 y}(\gamma(t))\right] \gamma_{2}^{\prime}(t)\right\} d t \\
& =\int_{0}^{1} \tilde{w}(Z(\gamma(t)))\left\{\left[Z_{1 x}(\gamma(t)) \gamma_{1}^{\prime}(t)+Z_{1 y}(\gamma(t)) \gamma_{2}^{\prime}(t)\right]+i\left[Z_{2 x}(\gamma(t)) \gamma_{1}^{\prime}(t)+Z_{2 y}(\gamma(t)) \gamma_{2}^{\prime}(t)\right]\right\} d t \\
& =\int_{0}^{1} \tilde{w}(Z(\gamma(t)))\left\{\frac{d}{d t}\left(Z_{1} \circ \gamma(t)\right)+i \frac{d}{d t}\left(Z_{2} \circ \gamma(t)\right)\right\} d t \\
& =\int_{Z \circ \gamma} \tilde{w}(\zeta)\{d \xi+i d \eta\}=\int_{Z \circ \gamma} \tilde{w}(\zeta) d \zeta .
\end{aligned}
$$

A próxima proposição nos dá uma fórmula de representação que generaliza para $L$ a fórmula de representação de Cauchy-Pompeiu ${ }^{1}$.

Proposição 17. Seja $U$ um aberto como no Lema 8. Seja $w \in C^{1}(\tilde{\Omega})$ e $Z: \tilde{\Omega} \rightarrow \mathbb{C}$ uma integral primeira global de $L$, de classe $C^{1+\varepsilon}$, que é um homeomorfismo sobre sua imagem. Então, para todo $(x, y) \in U$, temos

$$
w(x, y)=\frac{1}{2 \pi i} \int_{\partial U} \frac{w(\xi, \eta)}{Z(\xi, \eta)-Z(x, y)} d Z(\xi, \eta)-\frac{1}{2 \pi i} \int_{U} \frac{H_{Z} w(\xi, \eta)}{Z(\xi, \eta)-Z(x, y)} d \xi d \eta
$$

Demonstração. Seja $\left(x_{0}, y_{0}\right) \in U$ fixado. Considere $z_{0}=Z\left(x_{0}, y_{0}\right)$ e seja $\delta>0$ tal que $\overline{D_{\delta}} \subset$ $Z(U)$, onde $D_{\delta}=D\left(z_{0}, \delta\right)$. Defina $K_{\delta}=Z^{-1}\left(\overline{D_{\delta}}\right)$ e $U_{\delta}=U \backslash K_{\delta}$. Seja

$$
f(x, y)=\frac{w(x, y)}{Z(x, y)-z_{0}}
$$

1 A fórmula de representação de Cauchy-Pompeiu também é conhecida como fórmula de Cauchy não homogênea. Veja em (BEGEHR, 1994) e (VEKUA, 1962).Veja também o Teorema 31, página 138. 
Temos que $f$ é de classe $C^{1}$ em um aberto que contém $U_{\delta}$. Assim, pela Proposição 16 , fórmula (5.11),

$$
\begin{aligned}
\int_{U_{\delta}} \frac{H_{Z} w(x, y)}{Z(x, y)-z_{0}} d x d y & =\int_{\partial U_{\delta}} \frac{w(x, y)}{Z(x, y)-z_{0}} d Z(x, y) \\
& =\int_{\partial U} \frac{w(x, y)}{Z(x, y)-z_{0}} d Z(x, y)-\int_{\partial K_{\delta}} \frac{w(x, y)}{Z(x, y)-z_{0}} d Z(x, y)
\end{aligned}
$$

Pelo Lema 9, fórmula (5.13),

$$
\int_{\partial K_{\delta}} f(x, y) d Z(x, y)=\int_{Z\left(\partial K_{\delta}\right)}\left(f \circ Z^{-1}\right)(\zeta) d \zeta=\int_{\partial D_{\delta}} \frac{\tilde{w}(\zeta)}{\zeta-z_{0}} d \zeta
$$

onde $\tilde{w}=w \circ Z^{-1}$, então usando as coordenadas polares $\zeta=z_{0}+\delta e^{i \theta}, \theta \in[0,2 \pi]$, obtemos

$$
\int_{\partial D_{\delta}} \frac{\tilde{w}(\zeta)}{\zeta-z_{0}} d \zeta=\int_{0}^{2 \pi} \frac{\tilde{w}\left(z_{0}+\delta e^{i \theta}\right)}{\delta e^{i \theta}} i \delta e^{i \theta} d \theta \rightarrow 2 \pi i \tilde{w}\left(z_{0}\right), \text { quando } \delta \rightarrow 0 .
$$

Portanto,

$$
\int_{\partial K_{\delta}} \frac{w(x, y)}{Z(x, y)-z_{0}} d Z(x, y) \rightarrow 2 \pi i w\left(x_{0}, y_{0}\right), \text { quando } \delta \rightarrow 0
$$

Por outro lado, pelo Teorema 4 (página 72),

$$
(x, y) \mapsto \frac{1}{Z(x, y)-z_{0}} \in L^{1}(U) .
$$

Assim,

$$
\int_{U_{\delta}} \frac{H_{Z} w(x, y)}{Z(x, y)-z_{0}} d x d y \rightarrow \int_{U} \frac{H_{Z} w(x, y)}{Z(x, y)-z_{0}} d x d y, \quad \text { quando } \quad \delta \rightarrow 0 .
$$

O resultado segue de (5.14), (5.15) e (5.16).

Como anteriormente, seja $\Omega$ um aberto compactamente contido em $\tilde{\Omega}$.

Teorema 5. Se $f \in L^{1}(\Omega)$ então $T_{Z} f$ satisfaz $H_{Z}\left(T_{Z} f\right)=f$ em $\Omega$.

Demonstração. Seja $f \in L^{1}(\Omega)$. Pela Proposição 15, página 75, $T_{Z} f \in L^{1}(\Omega)$. Seja $\phi \in C_{c}^{\infty}(\Omega)$ uma função de classe $C^{\infty}$ com suporte compacto em $\Omega$. Existe um aberto simplesmente conexo $U$ com bordo suave de classe $C^{1}$ tal que $\bar{U}$ é compacto, $\bar{U} \subset \Omega$ e supp $\phi \subset U$. Logo,

$$
\int_{\partial U} \frac{\phi(x, y)}{Z(x, y)-Z(\xi, \eta)} d Z(x, y)=0
$$

para $(\xi, \eta) \in U$. Assim, pela Proposição 17, página 77, podemos escrever

$$
\phi(\xi, \eta)=-\frac{1}{2 \pi i} \int_{U} \frac{H_{Z} \phi(x, y)}{Z(x, y)-Z(\xi, \eta)} d x d y,
$$

para $(\xi, \eta) \in U$. Como supp $\phi \subset U$ segue que a representação de $\phi$ dada acima, em (5.17), vale para todo $(\xi, \eta) \in \Omega$. 
Temos

$$
\left\langle H_{Z}\left(T_{Z} f\right), \phi\right\rangle=\int_{\Omega} T_{Z} f(x, y)^{t} H_{Z} \phi(x, y) d x d y=-\int_{\Omega} T_{Z} f(x, y) H_{Z} \phi(x, y) d x d y .
$$

Daí, temos pelo Teorema de Fubini

$$
\begin{aligned}
\left\langle H_{Z}\left(T_{Z} f\right), \phi\right\rangle & =-\int_{\Omega} T_{Z} f(x, y) H_{Z} \phi(x, y) d x d y=-\int_{U} T_{Z} f(x, y) H_{Z} \phi(x, y) d x d y \\
& =-\frac{1}{2 \pi i} \int_{U}\left(\int_{\Omega} \frac{f(\xi, \eta)}{Z(\xi, \eta)-Z(x, y)} d \xi d \eta\right) H_{Z} \phi(x, y) d x d y \\
& =\int_{\Omega} f(\xi, \eta)\left(\frac{1}{2 \pi i} \int_{U} \frac{H_{Z} \phi(x, y)}{Z(x, y)-Z(\xi, \eta)} d x d y\right) d \xi d \eta \\
& =\int_{\Omega} f(\xi, \eta) \phi(\xi, \eta) d \xi d \eta=\langle f, \phi\rangle .
\end{aligned}
$$

Portanto, $H_{Z}\left(T_{Z} f\right)=f$ em $\Omega$.

Como $L$ é um campo vetorial complexo definido em $\tilde{\Omega}$ pertencente a classe $\mathscr{X}(\tilde{\Omega})$ e $Z: \tilde{\Omega} \rightarrow \mathbb{C}$ é uma integral primeira global de $L$, de classe $C^{1+\varepsilon}$, que é um homeomorfismo sobre sua imagem, podemos escrever o campo $L$ como um múltiplo do Hamiltoniano de $Z$, isto é, podemos escrever

$$
L=\lambda H_{Z} \operatorname{em} \tilde{\Omega},
$$

$\operatorname{com} \lambda \in C^{1}(\tilde{\Omega})$ e $|\lambda|>0$ em $\tilde{\Omega}$ (Proposição 9, página 49). Tendo isso em mente, vamos provar o corolário a seguir.

Como acima, seja $\Omega$ um aberto compactamente contido em $\Omega$.

Corolário 4. Se $f \in L^{1}(\Omega)$, então $u:=T_{Z}\left(\frac{f}{\lambda}\right)$, onde $\lambda$ é dado acima, satisfaz a equação $L u=f$ em $\Omega$.

Demonstração. Temos que $\lambda \in C^{1}(\tilde{\Omega})$. Como $\bar{\Omega}$ é compacto e $\bar{\Omega} \subset \tilde{\Omega}$ temos que $1 / \lambda \in$ $L^{\infty}(\Omega)$. $\operatorname{Logo} g:=f / \lambda \in L^{1}(\Omega)$. Pelo Teorema $5, H_{Z}\left(T_{Z} g\right)=g$ em $\Omega$. Como $\lambda \in C^{1}(\Omega)$ temos $\lambda H_{Z}\left(T_{Z} g\right)=\lambda g$ em $\Omega$, ou seja, $L\left(T_{Z} g\right)=f \operatorname{em} \Omega$.

O Teorema 4, página 72, nos diz que se $f \in L^{p}(\Omega), p>2+\sigma_{\mathrm{M}}\left(\sigma_{\mathrm{M}}>0\right.$ dado em (5.4), página 72), então $T_{Z} f \in L^{\infty}(\Omega)$. Assim, pelo corolário acima, se $f \in L^{p}(\Omega)$ para $p>2+\sigma_{\mathrm{M}}$, então existe uma solução $u \in L^{\infty}(\Omega)$ da equação $L u=f$ em $\Omega$. O próximo exemplo, explora a relação $p>2+\sigma_{\mathrm{M}}$ quando estamos procurando soluções limitadas da equação $L u=f$ em $\Omega$, $f \in L^{p}(\Omega)$.

Exemplo 6. Para $p \leq 2+\sigma_{\mathrm{M}}$, existe $f \in L^{p}(\Omega)$ tal que a equação $L u=f$ não tem soluções limitadas. Para o campo padrão,

$$
L_{\sigma}=\frac{\partial}{\partial y}-i|y| \frac{\partial}{\partial x}, \sigma>0
$$


a função $v: \bar{\Omega} \rightarrow \mathbb{C}$ definida por $v(x, y)=\ln |\ln | Z_{\sigma}(x, y) \|, \operatorname{com} Z_{\sigma}(x, y)=x+i \frac{y|y|}{\sigma+1}$, é não limitada, mas é solução da equação $L v=f$ com

$$
f(x, y)=\frac{-i|y|^{\sigma}}{\overline{Z_{\sigma}(x, y)} \ln \left|Z_{\sigma}(x, y)\right|} \in L^{p}(\Omega), \quad \text { para } \quad 1 \leq p \leq 2+\sigma .
$$

$\left(f \in L^{p}(\Omega)\right.$, com $p \leq 2+\sigma$, segue do fato de que $\int_{0}^{a} \frac{d r}{r^{s} \mid \ln r^{p}}<\infty, a>0$, se, e somente se, $s \leq 1)$. Para um campo mais geral, pertencente a classe $\mathscr{X}$, podemos construir um exemplo usando a normalização (estudada no Capítulo 3) perto dos pontos do seu conjunto característico.

\subsection{Continuidade Hölder das soluções de $L u=f$}

Seja $L$ um campo vetorial complexo definido em $\tilde{\Omega}$ pertencente à classe $\mathscr{X}(\tilde{\Omega})$ como no início do capítulo. Nesta seção vamos provar que as soluções contínuas de $L u=f$ são Hölder contínuas se $f \in L^{p}$, com $p>2+\sigma_{\mathrm{M}}$, onde $\sigma_{\mathrm{M}}>0$ é definido em (5.4), na página 72, como o máximo dos tipos $\sigma_{1}, \ldots, \sigma_{N}$ do campo $L$.

Seja $Z: \tilde{\Omega} \rightarrow \mathbb{C}$ uma integral primeira global de $L$, de classe $C^{1+\varepsilon}$, que é um homeomorfismo sobre sua imagem.

Como nas seções anteriores, seja $\Omega$ um aberto de $\Omega$ tal que $\bar{\Omega}$ é um compacto de $\mathbb{R}^{2}$ e $\bar{\Omega} \subset \tilde{\Omega}$. O próximo teorema nos diz que a função $T_{Z} f$ (dada em (5.5), na página 72 ) satisfaz uma condição de Hölder.

Teorema 6. Se $f \in L^{p}(\Omega)$, com $p>2+\sigma_{\mathrm{M}}$, então existe uma constante $C=C\left(p, \sigma_{1}, \ldots, \sigma_{N}, \Omega\right)>$ 0 tal que

$$
\left|T_{Z} f\left(\mathrm{p}_{1}\right)-T_{Z} f\left(\mathrm{p}_{2}\right)\right| \leq C|| f \|_{p}\left|Z\left(\mathrm{p}_{1}\right)-Z\left(\mathrm{p}_{2}\right)\right|^{\beta}, \quad \forall \mathrm{p}_{1}, \mathrm{p}_{2} \in \tilde{\Omega}
$$

onde $\beta=\left(2-q-\tau_{\mathrm{M}}\right) / q$, $\operatorname{com} q=p /(p-1)$ e $\tau_{\mathrm{M}}=\sigma_{\mathrm{M}} /\left(\sigma_{\mathrm{M}}+1\right)$.

Demonstração. Sejam $\left(x_{0}, y_{0}\right),\left(x_{1}, y_{1}\right) \in \tilde{\Omega}, \operatorname{com}\left(x_{0}, y_{0}\right) \neq\left(x_{1}, y_{1}\right)$. Temos, pela desigualdade de Hölder,

$$
\left|T_{Z} f\left(x_{1}, y_{1}\right)-T_{Z} f\left(x_{0}, y_{0}\right)\right| \leq \frac{\left|Z\left(x_{1}, y_{1}\right)-Z\left(x_{0}, y_{0}\right)\right|}{2 \pi}\|f\|_{p} J^{\frac{1}{q}}
$$

onde

$$
J=J\left(\left(x_{1}, y_{1}\right),\left(x_{0}, y_{0}\right)\right)=\int_{\Omega} \frac{d \xi d \eta}{\left|Z(\xi, \eta)-Z\left(x_{1}, y_{1}\right)\right|^{q}\left|Z(\xi, \eta)-Z\left(x_{0}, y_{0}\right)\right|^{q}}
$$

$\operatorname{com} q=p /(p-1)$.

Como no Teorema 4, página 72 , vamos estabelecer uma cobertura de $\bar{\Omega}$ onde podemos explorar as boas propriedades locais de $Z$.

Seja $\left\{V_{k}\right\}_{k=1, \ldots, k_{0}}$ uma cobertura finita do compacto $\Sigma \cap \bar{\Omega}$, formada por abertos pré compactos de $\tilde{\Omega}$, tais que cada aberto intercepta apenas uma componente conexa de $\Sigma$ (lembrando 
que $\Sigma=\Sigma_{1} \cup \ldots \cup \Sigma_{N}$, veja na página 100) e para cada $k$, com $V_{k} \cap \Sigma_{j} \neq \emptyset$, podemos escrever

$$
\left.Z\right|_{V_{k}}=G_{k} \circ Z_{\sigma_{j}} \circ F_{k} \mathrm{em} V_{k}
$$

onde $F_{k}: \overline{V_{k}} \rightarrow \overline{U_{k}}, G_{k}: \overline{Z_{\sigma_{j}}\left(U_{k}\right)} \rightarrow \overline{B_{k}}$ são difeomorfismos de classe $C^{1}$, e

$$
Z_{\sigma_{j}}(s, t)=s+i \frac{t|t|^{\sigma_{j}}}{1+\sigma_{j}} .
$$

Existe uma família de abertos de $\tilde{\Omega},\left\{V_{k}\right\}_{k=k_{0}+1, \ldots, m}$, com $\overline{V_{k}}$ compacto, $\overline{V_{k}} \cap \Sigma=\emptyset$, e tal que

$$
\bar{\Omega} \subset V_{1} \cup \ldots \cup V_{k_{0}} \cup V_{k_{0}+1} \cup \ldots \cup V_{m}
$$

isto é, $\mathscr{C}=\left\{V_{k}\right\}_{k=1, \ldots, m}$ é uma cobertura de $\bar{\Omega}$. Seja $\left\{\varphi_{k}\right\}_{k=1, \ldots, m}$ uma partição da unidade subordinada a cobertura $\mathscr{C}$. Assim, temos

$$
\begin{gathered}
J\left(\left(x_{1}, y_{1}\right),\left(x_{0}, y_{0}\right)\right)=\int_{\Omega} \frac{\sum_{k=1}^{m} \varphi_{k}(\xi, \eta) d \xi d \eta}{\left|Z(\xi, \eta)-Z\left(x_{1}, y_{1}\right)\right|^{q}\left|Z(\xi, \eta)-Z\left(x_{0}, y_{0}\right)\right|^{q}} \\
=\sum_{k=1}^{m} \int_{V_{k} \cap \Omega} \frac{\varphi_{k}(\xi, \eta) d \xi d \eta}{\left|Z(\xi, \eta)-Z\left(x_{1}, y_{1}\right)\right|^{q}\left|Z(\xi, \eta)-Z\left(x_{0}, y_{0}\right)\right|^{q}}=: \sum_{k=1}^{m} J_{k}\left(\left(x_{1}, y_{1}\right),\left(x_{0}, y_{0}\right)\right) .
\end{gathered}
$$

Vamos Estimar $J_{k}\left(\left(x_{1}, y_{1}\right),\left(x_{0}, y_{0}\right)\right)$, para $k \in\{1, \ldots, m\}$.

Suponha que $\left|Z\left(x_{1}, y_{1}\right)-Z\left(x_{0}, y_{0}\right)\right|<1$.

Caso 1: $\left(x_{0}, y_{0}\right),\left(x_{1}, y_{1}\right) \in V_{k}$ e $k \in\left\{1, \ldots, k_{0}\right\}$.

Seja $j \in\{1, \ldots, N\}$ tal que $V_{k} \cap \Sigma_{j} \neq 0$. Temos,

$$
\begin{gathered}
\int_{V_{k}} \frac{d \xi d \eta}{\left|Z(\xi, \eta)-Z\left(x_{1}, y_{1}\right)\right|^{q}\left|Z(\xi, \eta)-Z\left(x_{0}, y_{0}\right)\right|^{q}}= \\
=\int_{V_{k}} \frac{d \xi d \eta}{\left|G_{k}\left(Z_{\sigma_{j}}\left(F_{k}(\xi, \eta)\right)\right)-G_{k}\left(Z_{\sigma_{j}}\left(F_{k}\left(x_{1}, y_{1}\right)\right)\right)\right|^{q}\left|G_{k}\left(Z_{\sigma_{j}}\left(F_{k}(\xi, \eta)\right)\right)-G_{k}\left(Z_{\sigma_{j}}\left(F_{k}\left(x_{0}, y_{0}\right)\right)\right)\right|^{q}} \\
\leq K^{2} \int_{V_{k}} \frac{d \xi d \eta}{\left|Z_{\sigma_{j}}\left(F_{k}(\xi, \eta)\right)-Z_{\sigma_{j}}\left(F_{k}\left(x_{1}, y_{1}\right)\right)\right|^{q}\left|Z_{\sigma_{j}}\left(F_{k}(\xi, \eta)\right)-Z_{\sigma_{j}}\left(F_{k}\left(x_{0}, y_{0}\right)\right)\right|^{q}} \\
\leq K^{2} K_{1} \int_{F_{k}\left(V_{k}\right)} \frac{d s d t}{\left|Z_{\sigma_{j}}(s, t)-Z_{\sigma_{j}}\left(s_{1}^{\circ}, t_{1}^{\circ}\right)\right|^{q} \mid Z_{\sigma_{j}}(s, t)-Z_{\sigma_{j}}\left(s_{0}^{\circ}, t_{0}^{\circ}\right)^{q}}=: Q_{j k},
\end{gathered}
$$

onde $K$ é uma constante de Lipschitz para toda $G_{\ell}^{-1}$, isto é,

$$
\left|\mathrm{q}_{1}-\mathrm{q}_{2}\right| \leq K\left|G_{\ell}\left(\mathrm{q}_{1}\right)-G_{\ell}\left(\mathrm{q}_{2}\right)\right|, \mathrm{q}_{1}, \mathrm{q}_{2} \in \overline{B_{\ell}}
$$

para todo $\ell \in\left\{1, \ldots, k_{0}\right\}$, e $K_{1}>0$ é tal que $K_{1} \geq \sup \left\{\left|\operatorname{det}\left(D F_{\ell}\right)(\xi, \eta)\right|^{-1} ;(\xi, \eta) \in V_{\ell}\right\}$, para todo $\ell \in\left\{1, \ldots, k_{0}\right\}$, e $\left(s_{n}^{\circ}, t_{n}^{\circ}\right)=F_{k}\left(x_{n}, y_{n}\right), n=0,1$. 
Usando a mudança de coordenadas $\xi=s$ e $\eta=\frac{t|t|^{\sigma_{j}}}{\sigma_{j}+1}$ temos

$$
Q_{j k}=\frac{1}{\left(\sigma_{j}+1\right)^{\tau_{j}}} \int_{Z_{\sigma_{j}}\left(U_{k}\right)} \frac{d \xi d \eta}{|\eta|^{\tau_{j}\left|\zeta-w_{1}\right|^{q}\left|\zeta-w_{0}\right|^{q}}}, \zeta=\xi+i \eta
$$

onde $w_{n}=Z_{\sigma_{j}}\left(s_{n}, t_{n}\right), n=0,1$, e $\tau_{j}=\sigma_{j} /\left(\sigma_{j}+1\right)$. Podemos assumir, sem perda de generalidade, que $\eta_{0}>0$, (onde $w_{0}=\xi_{0}+i \eta_{0}$ ), e $w_{1}-w_{0}=\left|w_{1}-w_{0}\right| e^{i \varphi}$, com $0 \leq \varphi \leq \pi$. Temos,

$$
\begin{aligned}
Q_{j k} & \leq \frac{1}{\left(\sigma_{j}+1\right)^{\tau_{j}}} \int_{D\left(w_{0} ; \mathrm{d}\right)} \frac{d \xi d \eta}{|\eta|^{\tau_{j}\left|\zeta-w_{1}\right|^{q}\left|\zeta-w_{0}\right|^{q}}} \\
& =\frac{1}{\left(\sigma_{j}+1\right)^{\tau_{j}}} \int_{D(0 ; \mathrm{d})} \frac{d \xi d \eta}{\left.\left|\eta_{0}+\eta\right|^{\tau_{j}}\left|\zeta-\left(w_{1}-w_{0}\right)^{q}\right| \zeta\right|^{q}}
\end{aligned}
$$

onde $\mathrm{d} \geq \operatorname{diam}\left(Z_{\sigma_{n}}\left(U_{\ell}\right)\right)$, para todo $n \in\{1, \ldots, N\}$ e todo $\ell \in\left\{1, \ldots, k_{0}\right\}$ com $V_{\ell} \cap \Sigma_{n} \neq 0$. A seguir, usando a mudança de variáveis $\zeta=\left|w_{1}-w_{0}\right| \mu, \operatorname{com} \mu=a+i b$ e $w_{1}-w_{0}=\left|w_{1}-w_{0}\right| e^{i \varphi}$, obtemos

$$
Q_{j k} \leq \frac{1}{\left(\sigma_{j}+1\right)^{\tau_{j}}} \int_{D\left(0 ; \frac{d}{\left|w_{1}-w_{0}\right|}\right)} \frac{\left|w_{1}-w_{0}\right|^{2-\tau-2 q}}{\left|\frac{\eta_{0}}{\left|w_{1}-w_{0}\right|}+b\right|^{\tau}\left|\mu-e^{i \varphi}\right| q|\mu|^{q}} d a d b .
$$

Finalmente, as coordenadas polares $\mu=\zeta e^{i \varphi}, \zeta=r e^{i \theta}$ e $\gamma=\eta_{0} /\left|w_{1}-w_{0}\right|$ e o uso do Lema 7 (página 64), nos permite obter a estimativa

$$
\begin{aligned}
Q_{j k} & \leq \frac{\left|w_{1}-w_{0}\right|^{2-\tau_{j}-2 q}}{\left(\sigma_{j}+1\right)^{\tau_{j}}} \int_{0}^{2 \pi} \int_{0}^{\frac{\mathrm{d}}{\left|w_{1}-w_{0}\right|}} \frac{r d r d \theta}{|\gamma+r \sin (\theta+\varphi)|^{\tau_{j}} r^{q}\left|r e^{i \theta}-1\right|^{q}} d \xi d \eta \\
& \leq \frac{C\left(q, \tau_{j}\right)}{\left(\sigma_{j}+1\right)^{\tau_{j}}}\left|w_{1}-w_{0}\right|^{2-\tau_{j}-2 q} \leq \frac{C\left(q, \tau_{j}\right)}{\left(\sigma_{j}+1\right)^{\tau_{j}}}\left(K\left|G_{k}\left(w_{1}\right)-G_{k}\left(w_{0}\right)\right|\right)^{2-\tau_{j}-2 q} \\
& =\frac{C\left(q, \tau_{j}\right)}{\left(\sigma_{j}+1\right)^{\tau_{j}}} K^{2-\tau_{j}-2 q}\left|Z\left(x_{1}, y_{1}\right)-Z\left(x_{0}, y_{0}\right)\right|^{2-\tau_{j}-2 q} \\
& \leq \frac{C\left(q, \tau_{j}\right)}{\left(\sigma_{j}+1\right)^{\tau_{j}}} K^{2-\tau_{j}-2 q}\left|Z\left(x_{1}, y_{1}\right)-Z\left(x_{0}, y_{0}\right)\right|^{2-\tau_{\mathrm{M}}-2 q},
\end{aligned}
$$

pois, $p>2+\sigma_{\mathrm{M}}$ implica $1<q<2-\tau_{\mathrm{M}} \leq 2-\tau_{\ell}$, para todo $\ell \in\{1, \ldots, N\}$, e pela suposição de $\left|Z\left(x_{1}, y_{1}\right)-Z\left(x_{0}, y_{0}\right)\right|<1$.

Segue daí que existe uma constante $Q=Q\left(q, \sigma_{1}, \ldots, \sigma_{N}, \Omega\right)>0$ tal que

$$
\int_{V_{k}} \frac{d \xi d \eta}{\left|Z(\xi, \eta)-Z\left(x_{1}, y_{1}\right)\right|^{q}\left|Z(\xi, \eta)-Z\left(x_{0}, y_{0}\right)\right|^{q}} \leq Q\left|Z\left(x_{1}, y_{1}\right)-Z\left(x_{0}, y_{0}\right)\right|^{2-\tau_{\mathrm{M}}-2 q} .
$$

Assim, pelas estimativas feitas acima, existe uma constante $\tilde{Q}=\tilde{Q}\left(q, \sigma_{1}, \ldots, \sigma_{N}, \Omega\right)>0$ tal que

$$
J_{k}\left(\left(x_{1}, y_{1}\right),\left(x_{0}, y_{0}\right)\right) \leq \tilde{Q}\left|Z\left(x_{1}, y_{1}\right)-Z\left(x_{0}, y_{0}\right)\right|^{2-2 q-\tau_{\mathrm{M}}} .
$$


Caso 2: $\left(x_{0}, y_{0}\right),\left(x_{1}, y_{1}\right) \in V_{k}$ e $k \in\left\{k_{0}+1, \ldots, m\right\}$.

Temos

$$
\begin{aligned}
J_{k}\left(\left(x_{1}, y_{1}\right),\left(x_{0}, y_{0}\right)\right) & \leq \int_{V_{k}} \frac{d \xi d \eta}{\left|Z(\xi, \eta)-Z\left(x_{1}, y_{1}\right)\right|^{q}\left|Z(\xi, \eta)-Z\left(x_{0}, y_{0}\right)\right|^{q}} \\
& \leq C \int_{Z\left(V_{k}\right)} \frac{d \xi d \eta}{\left|\zeta-Z\left(x_{1}, y_{1}\right)\right|^{q}\left|\zeta-Z\left(x_{0}, y_{0}\right)\right|^{q}}, \zeta=\xi+i \eta
\end{aligned}
$$

onde $C>0$ é tal que $C \geq \sup \left\{|\operatorname{det}(D Z)(\xi, \eta)|^{-1} ;(\xi, \eta) \in V_{\ell}\right\}$, para todo $\ell \in\left\{k_{0}+1, \ldots, m\right\}$. Pela desigualdade de Hadamard (Lema 18, página 136) existe uma constante $M(q)>0$ tal que

$$
\int_{Z\left(V_{k}\right)} \frac{d \xi d \eta}{\left|\zeta-Z\left(x_{1}, y_{1}\right)\right|^{q}\left|\zeta-Z\left(x_{0}, y_{0}\right)\right|^{q}} \leq M(q)\left|Z\left(x_{1}, y_{1}\right)-Z\left(x_{0}, y_{0}\right)\right|^{2-2 q}
$$

Como $\left|Z\left(x_{1}, y_{1}\right)-Z\left(x_{0}, y_{0}\right)\right|<1$, temos $\left|Z\left(x_{1}, y_{1}\right)-Z\left(x_{0}, y_{0}\right)\right|^{2-2 q} \leq\left|Z\left(x_{1}, y_{1}\right)-Z\left(x_{0}, y_{0}\right)\right|^{2-2 q-\tau_{\mathrm{M}}}$. Assim, temos

$$
J_{k}\left(\left(x_{1}, y_{1}\right),\left(x_{0}, y_{0}\right)\right) \leq C M(q)\left|Z\left(x_{1}, y_{1}\right)-Z\left(x_{0}, y_{0}\right)\right|^{2-2 q-\tau_{\mathrm{M}}} .
$$

Caso 3: $\left(x_{0}, y_{0}\right) \in V_{k} \mathrm{e}\left(x_{1}, y_{1}\right) \in V_{k}^{c}$, para $k \in\{1, \ldots, m\}$.

Como $\operatorname{supp} \varphi_{\ell} \subset V_{\ell}$, para todo $\ell \in\{1, \ldots, m\}$, temos que existe $\mu>0$ tal que

$$
0<\mu \leq \inf \left\{|Z(\xi, \eta)-Z(x, y)| ;(\xi, \eta) \in \operatorname{supp} \varphi_{\ell},(x, y) \in V_{\ell}^{c}\right\}
$$

para todo $\ell \in\{1, \ldots, m\}$. Assim,

$$
\begin{aligned}
J_{k}\left(\left(x_{1}, y_{1}\right),\left(x_{0}, y_{0}\right)\right) & =\int_{V_{k} \cap \Omega} \frac{\varphi_{k}(\xi, \eta) d \xi d \eta}{\left|Z(\xi, \eta)-Z\left(x_{1}, y_{1}\right)\right|^{q}\left|Z(\xi, \eta)-Z\left(x_{0}, y_{0}\right)\right|^{q}} \\
& \leq \int_{\operatorname{supp} \varphi_{k} \cap \Omega} \frac{\varphi_{k}(\xi, \eta) d \xi d \eta}{\left|Z(\xi, \eta)-Z\left(x_{1}, y_{1}\right)\right|^{q}\left|Z(\xi, \eta)-Z\left(x_{0}, y_{0}\right)\right|^{q}} \\
& \leq \frac{1}{\mu^{q}} \int_{\operatorname{supp} \varphi_{k} \cap \Omega} \frac{d \xi d \eta}{\left|Z(\xi, \eta)-Z\left(x_{0}, y_{0}\right)\right|^{q}} \leq \frac{1}{\mu^{q}} \int_{V_{k}} \frac{d \xi d \eta}{\left|Z(\xi, \eta)-Z\left(x_{0}, y_{0}\right)\right|^{q}} \leq M_{0},
\end{aligned}
$$

por um argumento semelhante ao usado na demostração do Teorema 4, página 72.

Como $\left|Z\left(x_{1}, y_{1}\right)-Z\left(x_{0}, y_{0}\right)\right|<1$, temos

$$
1<\left|Z\left(x_{1}, y_{1}\right)-Z\left(x_{0}, y_{0}\right)\right|^{2-2 q-\tau_{\mathrm{M}}}
$$

pois, $2-2 q-\tau_{\mathrm{M}}<0$. Daí,

$$
J_{k}\left(\left(x_{1}, y_{1}\right),\left(x_{0}, y_{0}\right)\right) \leq M_{0}\left|Z\left(x_{1}, y_{1}\right)-Z\left(x_{0}, y_{0}\right)\right|^{2-2 q-\tau_{\mathrm{M}}}
$$


Caso 4: $\left(x_{0}, y_{0}\right) \in V_{k}^{c} \mathrm{e}\left(x_{1}, y_{1}\right) \in V_{k}$, para $k \in\{1, \ldots, m\}$.

Este caso é inteiramente análogo ao caso anterior.

Caso 5: $\left(x_{0}, y_{0}\right),\left(x_{1}, y_{1}\right) \in V_{k}^{c}$, para $k \in\{1, \ldots, m\}$.

Temos

$$
\begin{aligned}
J_{k}\left(\left(x_{1}, y_{1}\right),\left(x_{0}, y_{0}\right)\right) & =\int_{V_{k} \cap \Omega} \frac{\varphi_{k}(\xi, \eta) d \xi d \eta}{\left|Z(\xi, \eta)-Z\left(x_{1}, y_{1}\right)\right|^{q} \mid Z(\xi, \eta)-Z\left(x_{0}, y_{0}\right)^{q}} \\
& \leq \int_{\operatorname{supp} \varphi_{k} \cap \Omega} \frac{\varphi_{k}(\xi, \eta) d \xi d \eta}{\left|Z(\xi, \eta)-Z\left(x_{1}, y_{1}\right)\right|^{q}\left|Z(\xi, \eta)-Z\left(x_{0}, y_{0}\right)\right|^{q}} \\
& \leq \frac{\left|\operatorname{supp} \varphi_{k} \cap \Omega\right|}{\mu^{2 q}} \leq \frac{|\Omega|}{\mu^{2 q}}
\end{aligned}
$$

onde $\mu>0$ é dado em (5.22). Assim, usando (5.23), temos

$$
J_{k}\left(\left(x_{1}, y_{1}\right),\left(x_{0}, y_{0}\right)\right) \leq \frac{|\Omega|}{\mu^{2 q}}\left|Z\left(x_{1}, y_{1}\right)-Z\left(x_{0}, y_{0}\right)\right|^{2-2 q-\tau_{\mathrm{M}}} .
$$

Assim terminamos o caso 5.

Portanto, para $\left(x_{0}, y_{0}\right),\left(x_{1}, y_{1}\right) \in \tilde{\Omega}$, com $\left|Z\left(x_{1}, y_{1}\right)-Z\left(x_{0}, y_{0}\right)\right|<1$, existe uma constante $M_{1}=M_{1}\left(q, \sigma_{1}, \ldots, \sigma_{N}, \Omega\right)>0$ tal que

$$
J_{k}\left(\left(x_{1}, y_{1}\right),\left(x_{0}, y_{0}\right)\right) \leq M_{1}\left|Z\left(x_{1}, y_{1}\right)-Z\left(x_{0}, y_{0}\right)\right|^{2-2 q-\tau_{\mathrm{M}}}, \text { para todo } k \in\{1, \ldots, m\}
$$

Assim, por (5.18) e (5.21) temos

$$
\begin{aligned}
\left|T_{Z} f\left(x_{1}, y_{1}\right)-T_{Z} f\left(x_{0}, y_{0}\right)\right| & \leq \frac{\left|Z\left(x_{1}, y_{1}\right)-Z\left(x_{0}, y_{0}\right)\right|}{2 \pi}|| f||_{p}\left(m M_{1}\right)^{\frac{1}{q}}\left|Z\left(x_{1}, y_{1}\right)-Z\left(x_{0}, y_{0}\right)\right|^{\frac{2-2 q-\tau_{M}}{q}} \\
& \leq \frac{\left(m M_{1}\right)^{\frac{1}{q}}}{2 \pi}|| f||_{p}\left|Z\left(x_{1}, y_{1}\right)-Z\left(x_{0}, y_{0}\right)\right|^{\frac{2-q-\tau_{M}}{q}} .
\end{aligned}
$$

Agora, para $\left|Z\left(x_{1}, y_{1}\right)-Z\left(x_{0}, y_{0}\right)\right| \geq 1$, temos $\left|Z\left(x_{1}, y_{1}\right)-Z\left(x_{0}, y_{0}\right)\right|^{\frac{2-q-\tau_{M}}{q}} \geq 1 \mathrm{e}$

$$
\begin{aligned}
\left|T_{Z} f\left(x_{1}, y_{1}\right)-T_{Z} f\left(x_{0}, y_{0}\right)\right| & \leq\left|T_{Z} f\left(x_{1}, y_{1}\right)\right|+\left|T_{Z} f\left(x_{0}, y_{0}\right)\right| \\
& \leq\left. 2 M|| f\right|_{p} \\
& \leq 2 M|| f||_{p}\left|Z\left(x_{1}, y_{1}\right)-Z\left(x_{0}, y_{0}\right)\right|^{\frac{2-q-\tau_{M}}{q}} .
\end{aligned}
$$

onde $M>0$ é dada pelo Teorema 4 (págine 72). Tomando $C=\max \left\{2 M,\left(m M_{1}\right)^{\frac{1}{q}} / 2 \pi\right\}$, temos

$$
\left|T_{Z} f\left(x_{1}, y_{1}\right)-T_{Z} f\left(x_{0}, y_{0}\right)\right| \leq C|| f||_{p}\left|Z\left(x_{1}, y_{1}\right)-Z\left(x_{0}, y_{0}\right)\right|^{\frac{2-q-\tau_{M}}{q}}
$$

e isso completa a demonstração do teorema. 
Das propriedades relacionadas à hipocomplexidade de $L$ temos o seguinte corolário.

Corolário 5. Seja $f \in L^{p}(\Omega)$, com $p>2+\sigma_{\mathrm{M}}$. Se $u \in C(\Omega)$ satisfaz $L u=f$ em $\Omega$, então para todo aberto $U$ compactamente contido em $\Omega$ temos $u \in C^{\alpha}(U) \operatorname{com} \alpha=\left(2-q-\tau_{\mathrm{M}}\right) / q$, $\tau_{\mathrm{M}}=\sigma /\left(\sigma_{\mathrm{M}}+1\right)$ e $q=p /(p-1)$.

Demonstração. Sem perda de generalidade podemos supor que $L=H_{Z}$ em $\Omega$. Veja o Corolário 4 (página 79). Seja $u \in C(\Omega)$ tal que $L u=f$ em $\Omega$. Defina $v=u-T_{Z} f$. Temos $L v=0$ em $\Omega$. Pelo Teorema 2, página 53, podemos escrever $v=h \circ Z$ em $\Omega$, com $h: Z(\Omega) \rightarrow \mathbb{C}$ holomorfa. Assim,

$$
u=h(Z)+T_{Z} f \text { em } \Omega .
$$

Note que $T_{Z} f \in C^{\alpha}(\Omega)$ e $h \circ Z \in C^{\alpha}(U)$ para todo $U$ aberto compactamente contido em $\Omega$. Segue daí que $u \in C^{\alpha}(U)$.

O próximo corolário segue da demonstração do Teorema 6 e é uma generalização (de parte) da desigualdade de Hadamard para $Z$. (Veja a desigualdade de Hadamard clássica no Lema 18, página 136).

Corolário 6. Sejam $Z$ e $\Omega$ como no Teorema 6. Se $1 \leq q<2-\tau_{\mathrm{M}}$, então existe uma constante $M=M\left(q, \sigma_{1}, \ldots, \sigma_{N}, \Omega\right)>0$ tal que

$$
\int_{\Omega} \frac{d \xi d \eta}{\left|Z(\xi, \eta)-Z\left(x_{1}, y_{1}\right)\right|^{q}\left|Z(\xi, \eta)-Z\left(x_{0}, y_{0}\right)\right|^{q}} \leq M\left|Z\left(x_{1}, y_{1}\right)-Z\left(x_{0}, y_{0}\right)\right|^{2-\left(2 q+\tau_{\mathrm{M}}\right)},
$$

para todo $\left(x_{0}, y_{0}\right),\left(x_{1}, y_{1}\right) \in \tilde{\Omega}$.

Observação 23. Seja $0<\beta<1$ como no Teorema 6 (página 80 ). Considere $C_{Z}^{\beta}(\overline{\mathbf{\Omega}})$ como sendo o subconjunto de $C^{\beta}(\bar{\Omega})$ dado pelas funções $u$ tal que

$$
\left|u\left(\mathrm{p}_{1}\right)-u\left(\mathrm{p}_{2}\right)\right| \leq C\left|Z\left(\mathrm{p}_{1}\right)-Z\left(\mathrm{p}_{2}\right)\right|^{\beta}, \quad \forall \mathrm{p}_{1}, \mathrm{p}_{2} \in \bar{\Omega}
$$

para $C=C(u, \beta)>0$ constante. Pelo Teorema $6, T_{Z} f \in C_{Z}^{\beta}(\bar{\Omega})$, para $f \in L^{p}(\Omega)$, com $p>2+\sigma_{\mathrm{M}}$.

\subsection{Equação semilinear $L u=F(x, y, u)$}

Sejam $L$ um campo vetorial complexo definido no aberto $\tilde{\Omega}$ pertencente à classe $\mathscr{X}(\tilde{\Omega})$ como no início do capítulo. Seja $\Omega$ um aberto com $\bar{\Omega}$ compacto em $\mathbb{R}^{2}$ e $\bar{\Omega} \subset \tilde{\Omega}$. Nesta seção vamos estudar a equação semilinear

$$
L u=F(x, y, u) \text { em } \Omega,
$$

para $F: \bar{\Omega} \times \mathbb{C} \rightarrow \mathbb{C}$ em duas famílias funções. 
Seja $Z: \tilde{\Omega} \rightarrow \mathbb{C}$ uma integral primeira global de $L$, de classe $C^{1+\varepsilon}$, que é $Z$ um homeomorfismo sobre a imagem.

Para começar esta seção, vamos definir um conjunto que será de grande importância neste texto. Definimos o conjunto $\Lambda_{M, C}=\Lambda_{M, C}(Z, \Omega, \beta)$, para $M, C>0$ e $0<\beta<1$, como o conjunto

$$
\Lambda_{M, C}=\left\{v \in C(\bar{\Omega}) ;\|v\|_{\infty} \leq M,\left|v\left(\mathrm{p}_{1}\right)-v\left(\mathrm{p}_{2}\right)\right| \leq C\left|Z\left(\mathrm{p}_{1}\right)-Z\left(\mathrm{p}_{2}\right)\right|^{\beta}, \forall \mathrm{p}_{1}, \mathrm{p}_{2} \in \bar{\Omega}\right\}
$$

O conjunto $\Lambda_{M, C}$ é um subconjunto não vazio, fechado e convexo de $C(\bar{\Omega})$. Ademais, como consequência do Teorema de Arzelà-Ascoli, $\Lambda_{M, C}$ é compacto em $C(\bar{\Omega})$. Note também que $\Lambda_{M, C} \subset C_{Z}^{\beta}(\bar{\Omega})$.

Como no início do capítulo, seja

$$
\sigma_{\mathrm{M}}=\max _{1 \leq j \leq N}\left\{\sigma_{j}\right\}
$$

onde $\sigma_{1}, \cdots, \sigma_{N}$ são os respectivos tipos de $L$ ao longo das componentes conexas de $\Sigma$, a saber, $\Sigma_{1}, \cdots, \Sigma_{N}$. (Veja a Proposição 10, página 51, e a Observação 21, página 52).

Vamos tratar de dois tipos de equações semilineares. O primeiro tipo vem a seguir e o outro na página 100 , descrita por (6.5).

Sejam $\Psi \in L^{p}\left(\bar{\Omega} ; \overline{\mathbb{R}}_{+}\right), p>2+\sigma_{\mathrm{M}}$, e $0<\alpha \leq 1$. Definimos $\mathscr{F}_{\Psi}^{\alpha}$ como sendo o conjunto das funções $F: \bar{\Omega} \times \mathbb{C} \rightarrow \mathbb{C}$ que satisfazem

- $F(., \zeta) \in L^{p}(\bar{\Omega})$, para todo $\zeta \in \mathbb{C}$

- $\left|F\left(x, y, \zeta_{1}\right)-F\left(x, y, \zeta_{2}\right)\right| \leq \Psi(x, y)\left|\zeta_{1}-\zeta_{2}\right|^{\alpha}$, para todos $\zeta_{1}, \zeta_{2} \in \mathbb{C}$.

Observação 24. A família $\mathscr{F}_{\Psi}^{\alpha}$ é invariante por multiplicação por funções de $L^{\infty}(\Omega)$. Isto é, se $F \in \mathscr{F}_{\Psi}^{\alpha}$ e $\varphi \in L^{\infty}(\Omega)$, então $\varphi F \in \mathscr{F}_{\Psi}^{\alpha}$. Podemos escrever $L=\lambda H_{Z}$, com $\lambda \in C^{1}(\tilde{\Omega}) \mathrm{e}$ $|\lambda|>0$ em $\tilde{\Omega}$, onde $H_{Z}$ é o Hamiltoniano de $Z$ (Veja a Proposição 9, página 49). Em particular, $\lambda, 1 / \lambda \in C^{1}(\Omega) \cap L^{\infty}(\Omega)$. Assim, sem perda de generalidade, para estudar a existência de soluções para a equação $L u=F(x, y, u) \mathrm{em} \Omega$ podemos supor que $L=H_{Z} \mathrm{em} \tilde{\Omega}$. (Veja também a Observação 20, página 50).

Teorema 7. Seja $F \in \mathscr{F}_{\Psi}^{\alpha}$. Seja $\beta=\left(2-q-\tau_{\mathrm{M}}\right) / q$, sendo $\tau_{\mathrm{M}}=\sigma_{\mathrm{M}} /\left(\sigma_{\mathrm{M}}+1\right)$ e $q=p /(p-1)$, com $p$ e $\sigma_{\mathrm{M}}$ dados na definição da família $\mathscr{F}_{\Psi}^{\alpha}$. Então:

1. Se $0<\alpha<1$, a equação (5.24) tem solução $u \in C_{Z}^{\beta}(\bar{\Omega}) \subset C^{\beta}(\bar{\Omega})$.

2. Se $\alpha=1$ e a constante $M\left(p, \sigma_{1}, \ldots, \sigma_{N}, \Omega\right)$ que aparece no Teorema 4, página 72 , satisfaz

$$
M\left(p, \sigma_{1}, \ldots, \sigma_{N}, \Omega\right)\|\Psi\|_{p}<1,
$$

então (5.24) tem uma solução $u \in C_{Z}^{\beta}(\bar{\Omega}) \subset C^{\beta}(\bar{\Omega})$. 
Demonstração. Vamos supor $L=H_{Z}$ em $\tilde{\Omega}$ (Veja a Observação 24 acima).

Seja $C(\bar{\Omega})$ o espaço de Banach das funções contínuas em $\bar{\Omega}$ com a norma

$$
\|u\|_{\infty}=\sup \{|u(x, y)| ;(x, y) \in \bar{\Omega}\} .
$$

Para $M_{0}>0$, seja $C_{M_{0}}(\bar{\Omega})$ o subconjunto fechado de $C(\bar{\Omega})$ dado por

$$
C_{M_{0}}(\bar{\Omega})=\left\{u \in C(\bar{\Omega}) ;\|u\|_{\infty} \leq M_{0}\right\} .
$$

Suponha que $0<\alpha<1$. Então, para $M_{0}$ suficientemente grande, temos que

$$
M\left(p, \sigma_{1}, \ldots, \sigma_{N}, \Omega\right)\left\{\|\Psi\|_{p} M_{0}^{\alpha}+\|F(\cdot, 0)\|_{p}\right\} \leq M_{0},
$$

onde $M\left(p, \sigma_{1}, \ldots, \sigma_{N}, \Omega\right)>0$ é dado pelo Teorema 4, página 72 .

Considere o operador $P: C_{M_{0}}(\bar{\Omega}) \rightarrow C_{M_{0}}(\bar{\Omega})$ definido por

$$
P u=T_{Z}(F(\cdot, u)),
$$

onde $T_{Z}$ é dado por (5.5). O operador $P$ é bem definido. De fato, para $F \in \mathscr{F}_{\Psi}^{\alpha}$ e $u \in C_{M_{0}}(\bar{\Omega})$ temos

$$
\|F(\cdot, u)\|_{p} \leq\|\Psi\|_{p} M_{0}^{\alpha}+\|F(\cdot, 0)\|_{p},
$$

e segue de (5.27), (5.28) e do Teorema 4 que para $u \in C_{M_{0}}(\bar{\Omega})$, temos

$$
|P u(x, y)| \leq M\left(p, \sigma_{1}, \ldots, \sigma_{N}, \Omega\right)\left\{\|\Psi\|_{p} M_{0}^{\alpha}+\|F(\cdot, 0)\|_{p}\right\} \leq M_{0} .
$$

Além disso, para $\left(x_{1}, y_{1}\right),\left(x_{2}, y_{2}\right) \in \bar{\Omega}$, temos

$$
\begin{aligned}
\left|P u\left(x_{1}, y_{1}\right)-P u\left(x_{2}, y_{2}\right)\right| & \leq\left|T_{Z}(F(\cdot, u))\left(x_{1}, y_{1}\right)-T_{Z}(F(\cdot, u))\left(x_{2}, y_{2}\right)\right| \\
& \leq\left. C\left(p, \sigma_{1}, \ldots, \sigma_{N}, \Omega\right)|| F(\cdot, u)\right|_{p}\left|Z\left(x_{1}, y_{1}\right)-Z\left(x_{2}, y_{2}\right)\right|^{\beta} \\
& \leq C\left(p, \sigma_{1}, \ldots, \sigma_{N}, \Omega\right)\left\{\left.|| \Psi\right|_{p} M_{0}^{\alpha}+|| F(\cdot, 0)||_{p}\right\}\left|Z\left(x_{1}, y_{1}\right)-Z\left(x_{2}, y_{2}\right)\right|^{\beta} \\
& \leq C_{0}\left|Z\left(x_{1}, y_{1}\right)-Z\left(x_{2}, y_{2}\right)\right|^{\beta},
\end{aligned}
$$

onde $C_{0}:=C\left(p, \sigma_{1}, \ldots, \sigma_{N}, \Omega\right)\left\{\|\Psi\|_{p} M_{0}^{\alpha}+\|F(\cdot, 0)\|_{p}\right\}$ e $C\left(p, \sigma_{1}, \ldots, \sigma_{N}, \Omega\right)>0$ é dado pelo Teorema 6, página 80.

Temos assim que $P u \in \Lambda_{M_{0}, C_{0}}$, para todo $u \in C_{M_{0}}(\bar{\Omega})$.

O operador $P$ é contínuo. De fato, para $u, v \in C_{M_{0}}(\bar{\Omega})$, temos

$$
\begin{aligned}
|P u(x, y)-P v(x, y)| & \leq\left|T_{Z}(F(\cdot, u)-F(\cdot, v))(x, y)\right| \\
& \leq M\left(p, \sigma_{1}, \ldots, \sigma_{N}, \Omega\right)|| F(\cdot, u)-F(\cdot, v) \|_{p} \\
& \leq M\left(p, \sigma_{1}, \ldots, \sigma_{N}, \Omega\right)\|\Psi\|_{p}\|u-v\|_{\infty}^{\alpha} .
\end{aligned}
$$

Assim, a restrição $P: \Lambda_{M_{0}, C_{0}} \rightarrow \Lambda_{M_{0}, C_{0}}$ é contínua. Portanto, pelo Teorema do Ponto Fixo de Schauder (Teorema 26, página 133), existe $u \in \Lambda_{M_{0}, C_{0}}$ tal que $P u=u$. O ponto fixo

$$
u=T_{Z}(F(\cdot, u)) \in C_{Z}^{\beta}(\bar{\Omega})
$$


satisfaz $L u(x, y)=F(x, y, u(x, y)),(x, y) \in \Omega$ (Teorema 5, página 78).

Agora, suponha que $\alpha=1$ e $M\left(p, \sigma_{1}, \ldots \sigma_{N}, \Omega\right)\|\Psi\|_{p}<1$. Se $M_{0}>0$ é tomado suficientemente grande, a desigualdade (5.27) vale. Deste modo, o mesmo argumento usado quando $\alpha<1$, mostra a existência de $u \in C_{Z}^{\beta}(\bar{\Omega})$ satisfazendo (5.24) em $\Omega$.

Agora, considere $F$ dada por

$$
F(x, y, \zeta)=g(x, y) H(x, y, \zeta)+f(x, y)
$$

onde $f, g \in L^{p}(\Omega), p>2+\sigma_{\mathrm{M}}$, e $H: \bar{\Omega} \times \mathbb{C} \rightarrow \mathbb{C}$ é contínua e limitada, com $\|H\|_{\infty}<K$ para alguma constante positiva $K$.

Teorema 8. Seja $F$ dada por (6.5). Então, a equação $L u=F(x, y, u)$ tem uma solução $u \in C_{Z}^{\beta}(\bar{\Omega})$ ( $\beta$ como no teorema anterior).

Demonstração. Como no teorema anterior, seguindo a linha de pensamento da Observação 24, vamos supor $L=H_{Z}$ em $\tilde{\Omega}$.

Considere o operador $P: C(\bar{\Omega}) \rightarrow C(\bar{\Omega})$ definido por

$$
P u=T_{Z}(g H(\cdot, u)+f)
$$

Como $H(\cdot, u) \in L^{\infty}(\Omega)$ temos $g H(\cdot, u)+f \in L^{p}(\Omega), p>2+\sigma_{\mathrm{M}}$. Segue do Teorema 4, página 72 , que para todo $(x, y) \in \bar{\Omega}$ temos

$$
\begin{aligned}
|P u(x, y)| & \leq M\left(p, \sigma_{1}, \ldots, \sigma_{N}, \Omega\right)\|g H(\cdot, u)+f\|_{p} \\
& \leq M\left(p, \sigma_{1}, \ldots, \sigma_{N}, \Omega\right)\left\{\|g\|_{p}\|H(\cdot, u)\|_{\infty}+\|f\|_{p}\right\} \\
& \leq M\left(p, \sigma_{1}, \ldots, \sigma_{N}, \Omega\right)\left\{\|g\|_{p} K+\|f\|_{p}\right\}=: M_{0} .
\end{aligned}
$$

Além disso, segue do Teorema 6, página 80, que para $\left(x_{1}, y_{1}\right),\left(x_{2}, y_{2}\right) \in \bar{\Omega}$ e $u \in C(\bar{\Omega})$ existe uma constante $C\left(p, \sigma_{1}, \ldots, \sigma_{N}, \Omega\right)>0$ tal que

$$
\begin{aligned}
\left|P u\left(x_{1}, y_{1}\right)-P u\left(x_{2}, y_{2}\right)\right| & \leq\left|T_{Z}(g H(\cdot, u)+f)\left(x_{1}, y_{1}\right)-T_{Z}(g H(\cdot, u)+f)\left(x_{2}, y_{2}\right)\right| \\
& \leq C\left(p, \sigma_{1}, \ldots, \sigma_{N}, \Omega\right)|| g H(\cdot, u)+f||_{p}\left|Z\left(x_{1}, y_{1}\right)-Z\left(x_{2}, y_{2}\right)\right|^{\beta} \\
& \leq C\left(p, \sigma_{1}, \ldots, \sigma_{N}, \Omega\right)\left\{\|g\|_{p} K+|| f \|_{p}\right\}\left|Z\left(x_{1}, y_{1}\right)-Z\left(x_{2}, y_{2}\right)\right|^{\beta} .
\end{aligned}
$$

Tomando $C_{0}=C\left(p, \sigma_{1}, \ldots, \sigma_{N}, \Omega\right)\left\{\|g\|_{p} K+\|f\|_{p}\right\}$, obtemos $P u \in \Lambda_{M_{0}, C_{0}}$, para todo $u \in C(\bar{\Omega})$. O operador $P: \Lambda_{M_{0}, C_{0}} \rightarrow \Lambda_{M_{0}, C_{0}}$ é contínuo. De fato, $H$ é uniformemente contínua no conjunto compacto $U=\bar{\Omega} \times\left\{\zeta \in \mathbb{C} ;|\zeta| \leq M_{0}\right\}$; logo, dado $\varepsilon>0$, existe $\delta>0$ tal que

$$
\left|H\left(x, y, \zeta_{1}\right)-H\left(x, y, \zeta_{2}\right)\right|<\frac{\varepsilon}{M\left(p, \sigma_{1}, \ldots, \sigma_{N}, \Omega\right)\left\{\|g\|_{p}+1\right\}}
$$


para todo $(x, y) \in \bar{\Omega} \mathrm{e}\left|\zeta_{1}-\zeta_{2}\right|<\delta$. Assim, para $u, v \in \Lambda_{M_{0}, C_{0}} \operatorname{com}\|u-v\|_{\infty}<\delta$, temos

$$
\begin{aligned}
|P u(x, y)-P v(x, y)| & \leq\left|T_{Z}(g\{H(\cdot, u)-H(\cdot, v)(x, y)\})\right| \\
& \leq M\left(p, \sigma_{1}, \ldots, \sigma_{N}, \Omega\right)\|g\{H(\cdot, u)-H(\cdot, v)\}\|_{p} \\
& \leq M\left(p, \sigma_{1}, \ldots, \sigma_{N}, \Omega\right)\|g\|_{p}\|H(\cdot, u)-H(\cdot, v)\|_{\infty} \\
& \leq M\left(p, \sigma_{1}, \ldots, \sigma_{N}, \Omega\right)\|g\|_{p} \frac{\varepsilon}{M\left(p, \sigma_{1}, \ldots, \sigma_{N}, \Omega\right)\left\{\|g\|_{p}+1\right\}}<\varepsilon .
\end{aligned}
$$

Portanto, pelo Teorema do Ponto Fixo de Schauder (Teorema 26, página 133), $P$ possui um ponto fixo em $\Lambda_{M_{0}, C_{0}}$ que satisfaz a conclusão do teorema.

\subsection{Princípio da Similaridade}

Seja $\tilde{\Omega}$ um aberto de $\mathbb{R}^{2}$ e seja $L$ um campo vetorial complexo definido em $\tilde{\Omega}$ que pertence à classe $\mathscr{X}(\tilde{\Omega})$ (descrita na página 71 . Veja também o Capítulo 3 ).

Seja $\Omega$ um aberto compactamente contido em $\Omega$. Nesta seção vamos estudar as soluções contínuas da equação

$$
L u=a u+b \bar{u} \text { em } \Omega,
$$

onde $a, b \in L^{p}(\Omega)$. Mais precisamente, iremos provar (na página 96) uma versão do teorema que vem a seguir para a equação (5.31).

Teorema 9. (Princípio da Similaridade) Seja $D$ um aberto limitado de $\mathbb{C}$. Sejam $a, b \in L^{p}(D)$, $p>2$. Se $u \in C(D)$ é solução da equação

$$
\frac{\partial u}{\partial \bar{z}}=a u+b \bar{u} e m D
$$

então existe uma função h holomorfa em $D$ e uma função Hölder contínua $s \in C^{\beta}(\bar{D}), \beta=$ $(2-q) / q, q=p /(p-1)$, tal que

$$
u(z)=h(z) e^{s(z)}, \quad \forall z \in D
$$

Veja a demonstração deste resultado em (COURANT; HILBERT, 1962), (BEGEHR, 1994) e (VEKUA, 1962). As funções que satisfazem a equação (5.32) são chamadas de funções analíticas generalizadas (ou funções pseudo-analíticas). Tais funções, são objeto de estudo de vários trabalhos pelo fato de compartilharem muitas propriedades com funções analíticas (funções holomorfas de uma variável complexa). Veja também (BERS, 1956).

Em geral, o princípio de similaridade é válido para campos vetoriais elíticos, pois, dado um campo vetorial elítico $L$, é possível obter uma mudança de variável que torna $L$ um múltiplo (não singular) do operador de Cauchy-Riemann $\partial / \partial \bar{z}$ (veja a Observação 9 na página 32).

O corolário a seguir segue a linha do comentário acima. 
Corolário 7. Seja $L$ um campo elítico de classe $C^{1}$ em $\Omega$ e seja $Z: V \rightarrow \mathbb{C}, \operatorname{com} V$ um aberto de $\tilde{\Omega}$, uma integral primeira, de classe $C^{2}$, de $L$ em $V$. Se $u \in C(U)$, com $U$ um aberto compactamente contido em $V$, tal que $L u=a u+b \bar{u} \operatorname{em} U, \operatorname{com} a, b \in L^{p}(U), p>2$, então existe uma função holomorfa $h: Z(U) \rightarrow \mathbb{C}$ e uma função Hölder contínua $s \in C^{\beta}(Z(\bar{U}))$, com $\beta=(2-q) / q$, $q=p /(p-1)$, tais que

$$
u(x, y)=h(Z(x, y)) e^{s(Z(x, y))} \text { para }(x, y) \in U
$$

Demonstração. Podemos escrever $L$ da forma

$$
L=\lambda H_{Z} \text { em } V
$$

onde $H_{Z}$ é o Hamiltoniano de $Z$ e $\lambda$ é uma função de classe $C^{1}$ em $V$ tal que $|\lambda|>0$ em $V$ (Proposição 4, página 33). Daí, $1 / \lambda \in L^{\infty}(U) \cap C^{1}(U)$ e $a / \lambda, b / \lambda \in L^{p}(\Omega), p>2$.

Logo, como $L u=a u+b \bar{u}$ em $U$, dado $\psi \in C_{c}^{\infty}(Z(U))$, temos

$$
\left\langle H_{Z} u, \phi\right\rangle=\left\langle\frac{a}{\lambda} u+\frac{b}{\lambda} \bar{u}, \phi\right\rangle, \text { para } \phi:=\psi \circ Z \text {. }
$$

Como $L$ é elítico, temos que $\operatorname{det}(D Z)(x, y) \neq 0$ para todo $(x, y) \in V$. Daí, podemos usar $Z$ para fazer mudança de coordenadas. Temos

$$
\left\langle H_{Z} u, \phi\right\rangle=-\int_{U} u(x, y) H_{Z}(x, y) d x d y=-2 i c \int_{Z(U)}\left(u \circ Z^{-1}\right)(\zeta) \partial_{\zeta} \psi(\zeta) d \xi d \eta
$$

onde $c=-1$ ou 1 e $\zeta=\xi+i \eta$. (Veja a demonstração da Proposição 5, página 34). Por outro lado,

$$
\left\langle\frac{a}{\lambda} u+\frac{b}{\lambda} \bar{u}, \phi\right\rangle=\int_{U}\left(\frac{a}{\lambda} u+\frac{b}{\lambda} \bar{u}\right)(x, y) \phi(x, y) d x d y=\int_{Z(U)}\left(\tilde{a}\left(u \circ Z^{-1}\right)+\tilde{b} \overline{\left(u \circ Z^{-1}\right)}\right)(\zeta) \psi(\zeta) d \xi d \eta
$$

onde $\tilde{a}=\left(\frac{a}{\lambda \operatorname{det}(D Z)}\right) \circ Z^{-1}, \tilde{b}=\left(\frac{b}{\lambda \operatorname{det}(D Z)}\right) \circ Z^{-1}$. Segue daí que

$$
\partial_{\bar{z}}\left(u \circ F^{-1}\right)=\frac{\tilde{a}}{2 i c}\left(u \circ F^{-1}\right)+\frac{\tilde{b}}{2 i c} \overline{\left(u \circ F^{-1}\right)} \operatorname{em} Z(U) .
$$

Note que $\tilde{a} / 2 i c, \tilde{b} / 2 i c \in L^{p}(Z(U))$. Pelo Teorema 9 existem $h$ holomorfa em $Z(U)$ e $s \in$ $C^{\beta}(Z(\bar{U})), \operatorname{com} \beta=(2-q) / q, q=p /(p-1)$, tais que

$$
\left(u \circ Z^{-1}\right)(\zeta)=h(\zeta) e^{s(\zeta)} \text {, para todo } \zeta \in Z(U) .
$$

Compondo a igualdade acima com $Z$ obtemos o resultado.

\subsubsection{Lemas de diferenciação}

Seja $\tilde{\Omega}$ um aberto conexo de $\mathbb{R}^{2}$ e seja $\Omega$ um aberto conexo compactamente contido em $\tilde{\Omega}$, isto é, $\bar{\Omega}$ é um compacto de $\mathbb{R}^{2}$ e $\bar{\Omega} \subset \tilde{\Omega}$. Considere o campo vetorial

$$
L=A \frac{\partial}{\partial x}+B \frac{\partial}{\partial y} \operatorname{em} \tilde{\Omega}
$$


sendo $A, B \in C^{1}(\tilde{\Omega})$.

Seja $\phi \in C_{c}^{\infty}\left(\mathbb{R}^{2}\right)$, com $\operatorname{supp}(\phi) \subset\left\{\mathrm{p} \in \mathbb{R}^{2} ;|\mathrm{p}| \leq 1\right\}, \phi \geq 0$ e $\int_{\mathbb{R}^{2}} \phi(\mathrm{p}) d \mathrm{p}=1$. Para $\varepsilon>0$ considere $\phi_{\varepsilon}(\mathrm{p})=\phi(\mathrm{p} / \varepsilon) \varepsilon^{-2}$. Para $u \in L_{l o c}^{1}\left(\mathbb{R}^{2}\right)$ definimos

$$
u_{\varepsilon}(\mathrm{p}):=u * \phi_{\varepsilon}(\mathrm{p})=\int_{\mathbb{R}^{2}} u(\mathrm{p}-\mathrm{q}) \phi_{\varepsilon}(\mathrm{q}) d \mathrm{q}, \mathrm{p} \in \mathbb{R}^{2}
$$

Temos que $u_{\varepsilon} \in C^{\infty}\left(\mathbb{R}^{2}\right)$ e $\operatorname{supp}\left(u_{\varepsilon}\right)=\left\{\mathrm{p} \in \mathbb{R}^{2} ;|\mathrm{p}| \leq \varepsilon\right\}+\operatorname{supp}(u)$.

Lema 10. - Se $u \in C_{c}^{k}\left(\mathbb{R}^{2}\right), k=0,1,2, \ldots$, então para $0 \leq m+n \leq k$, temos

$$
\left\|\frac{\partial^{m+n} u_{\varepsilon}}{\partial x^{m} \partial y^{n}}-\frac{\partial^{m+n} u}{\partial x^{m} \partial y^{n}}\right\|_{\infty} \rightarrow 0 \text { quando } \varepsilon \rightarrow 0 ;
$$

- Se $u \in L^{p}\left(\mathbb{R}^{2}\right), 1 \leq p<\infty$, então $\left\|u_{\varepsilon}-u\right\|_{p} \rightarrow 0$ quando $\varepsilon \rightarrow 0$.

Veja (HöRMANDER, a).

Vamos utilizar um caso particular do Lema de Friedrichs, o qual pode ser visto em (HöRMANDER, b):

Lema 11. (Friedrichs) Seja $v \in L^{p}\left(\mathbb{R}^{2}\right), 1 \leq p<\infty$, e $a \in C_{c}^{1}\left(\mathbb{R}^{2}\right)$. Então

$$
\left\|\left(a \partial_{j} v\right) * \phi_{\varepsilon}-a \partial_{j}\left(v * \phi_{\varepsilon}\right)\right\|_{p, \Omega} \rightarrow 0 \text { quando } \varepsilon \rightarrow 0 \text {. }
$$

Seja $\chi \in C_{c}^{\infty}\left(\mathbb{R}^{2}\right)$ tal que $\chi=1$ in $\bar{\Omega}$ e $\chi=0$ in $\mathbb{R}^{2} \backslash \tilde{\Omega}$. Considere

$$
\tilde{A}=\chi A \text { e } \tilde{B}=\chi B
$$

onde $A$ e $B$ são os coeficientes do campo $L$ dado no início da seção. Defina

$$
\tilde{L}=\tilde{A} \frac{\partial}{\partial x}+\tilde{B} \frac{\partial}{\partial y} \mathrm{em} \mathbb{R}^{2}
$$

Observe que $\tilde{L}=L$ em $\Omega$ e $\tilde{A}, \tilde{B} \in C_{c}^{1}\left(\mathbb{R}^{2}\right)$.

Corolário 8. Seja $v \in L^{\infty}(\Omega)$ tal que $L(v) \in L^{p}(\Omega), 1 \leq p<\infty$. Então

$$
\left\|L(v)-L\left(v * \phi_{\varepsilon}\right)\right\|_{p, \Omega} \rightarrow 0, \text { quando } \varepsilon \rightarrow 0 .
$$


Demonstração. Podemos supor que $v \in L^{p}\left(\mathbb{R}^{2}\right)$ tomando $v=0$ em $\mathbb{R}^{2} \backslash \Omega$. Temos

$$
\begin{gathered}
\left\|L v-L\left(v * \phi_{\varepsilon}\right)\right\|_{p, \Omega}=\left\|\tilde{L} v-\tilde{L}\left(v * \phi_{\varepsilon}\right)\right\|_{p, \Omega} \\
\leq\left\|\tilde{L}(v)-(\tilde{L}(v)) * \phi_{\varepsilon}\right\|_{p, \Omega}+\left\|(\tilde{L}(v)) * \phi_{\varepsilon}-\tilde{L}\left(v * \phi_{\varepsilon}\right)\right\|_{p, \Omega} .
\end{gathered}
$$

$\mathrm{O}$ resultado segue da desigualdade acima. De fato, analisando os termos da desigualdade acima temos que

$$
\left\|\tilde{L}(v)-(\tilde{L}(v)) * \phi_{\varepsilon}\right\|_{p, \Omega} \rightarrow 0 \text { quando } \varepsilon \rightarrow 0,
$$

pelo Lema 10. Temos também que

$$
\left\|(\tilde{L}(v)) * \phi_{\varepsilon}-\tilde{L}\left(v * \phi_{\varepsilon}\right)\right\|_{p, \Omega} \rightarrow 0 \text { quando } \varepsilon \rightarrow 0
$$

como uma consequência do Lema 11 devido à seguinte estimativa:

$$
\begin{gathered}
\left\|(\tilde{L} v) * \phi_{\varepsilon}-\tilde{L}\left(v * \phi_{\varepsilon}\right)\right\|_{p, \Omega}=\left\|\left(\tilde{A} \partial_{x} v\right) * \phi_{\varepsilon}+\left(\tilde{B} \partial_{y} v\right) * \phi_{\varepsilon}-\tilde{A} \partial_{x}\left(v * \phi_{\varepsilon}\right)-\tilde{B} \partial_{x}\left(v * \phi_{\varepsilon}\right)\right\|_{p, \Omega} \\
\leq\left\|\left(\tilde{A} \partial_{x} v\right) * \phi_{\varepsilon}-\tilde{A} \partial_{x}\left(v * \phi_{\varepsilon}\right)\right\|_{p, \Omega}+\left\|\left(\tilde{B} \partial_{y} v\right) * \phi_{\varepsilon}-\tilde{B} \partial_{x}\left(v * \phi_{\varepsilon}\right)\right\|_{p, \Omega} .
\end{gathered}
$$

Os Lemas 12 e 13 que veremos a seguir são versões mais simples, em certo sentido, de um lema de (BERHANU; HOUNIE; SANTIAGO, 2001).

Lema 12. Sejam $u, v \in L^{\infty}(\Omega)$ tais que $f:=L u, g:=L v \in L^{p}(\Omega), 1 \leq p<\infty$. Então,

$$
L(u v)=f v+u g \operatorname{em} \Omega
$$

Demonstração. Podemos supor $u, v \in L^{\infty}\left(\mathbb{R}^{2}\right)$ tomando $u=v=0$ em $\mathbb{R}^{2} \backslash \Omega$. Considere as regularizações,

$$
u_{\varepsilon}=\phi_{\varepsilon} * u \text { e } v_{\varepsilon}=\phi_{\varepsilon} * v .
$$

Seja $f_{\varepsilon}:=L\left(u_{\varepsilon}\right)$ e $g_{\varepsilon}=L\left(v_{\varepsilon}\right)$. Pelo Corolário 8 ,

$$
f_{\varepsilon} \rightarrow f \text { e } g_{\varepsilon} \rightarrow g \text { em } L^{p}(\Omega)
$$

Podemos escrever

$$
u g-u_{\varepsilon} g_{\varepsilon}=u\left(g-g_{\varepsilon}\right)+\left(u-u_{\varepsilon}\right) g_{\varepsilon} .
$$

Logo,

$$
\left\|u g-u_{\varepsilon} g_{\varepsilon}\right\|_{p} \leq\|u\|_{\infty}\left\|g-g_{\varepsilon}\right\|_{p}+\left\|u-u_{\varepsilon}\right\|_{\infty}\left\|g_{\varepsilon}\right\|_{p}
$$

lembrando que $g_{\varepsilon}$ converge para $g$ em $L^{p}(\Omega)$, temos que existe uma constante $M>0$ tal que $\left\|g_{\mathcal{\varepsilon}}\right\|_{p} \leq M$; assim, pela desigualdade (5.34) segue que

$$
u_{\varepsilon} g_{\varepsilon} \rightarrow u g \text { em } L^{p}(\Omega)
$$


Da mesma forma, temos que

$$
v_{\varepsilon} f_{\varepsilon} \rightarrow v f \mathrm{em} L^{p}(\Omega) .
$$

Como,

$$
\left\|L\left(u_{\varepsilon} v_{\varepsilon}\right)-f v-u g\right\|_{p} \leq\left\|f_{\varepsilon} v_{\varepsilon}-f v\right\|_{p}+\left\|u_{\varepsilon} g_{\varepsilon}-u g\right\|_{p},
$$

por (5.35) e (5.36) temos

$$
L\left(u_{\varepsilon} v_{\varepsilon}\right) \rightarrow f v+u g \text { em } L^{p}(\Omega)
$$

Dado $\varphi \in C_{c}^{1}(\Omega)$, temos

$$
\begin{gathered}
\left|\left\langle L\left(u_{\varepsilon} v_{\varepsilon}\right)-(f v+u g), \varphi\right\rangle\right| \leq \int_{\Omega}\left|\left(L\left(u_{\varepsilon} v_{\varepsilon}\right)-f v-u g\right)(\xi, \eta) \varphi(\xi, \eta)\right| d \xi d \eta \\
\leq\|\varphi\|_{q}\left\|L\left(u_{\varepsilon} v_{\varepsilon}\right)-f v-u g\right\|_{p},
\end{gathered}
$$

onde $1 / p+1 / q=1$. Assim,

$$
\left|\left\langle L\left(u_{\varepsilon} v_{\varepsilon}\right)-(f v+u g), \varphi\right\rangle\right| \rightarrow 0 \text { quando } \varepsilon \rightarrow 0 .
$$

Portanto,

$$
L\left(u_{\varepsilon} v_{\varepsilon}\right) \rightarrow f v+u g \text { em } \mathscr{D}^{\prime}(\Omega) .
$$

Por outro lado, dada $\varphi \in C_{c}^{1}(\Omega)$,

$$
\begin{gathered}
\left|\left\langle L\left(u_{\varepsilon} v_{\varepsilon}\right)-L(u v), \varphi\right\rangle\right|=\left|\left\langle L\left(u_{\varepsilon} v_{\varepsilon}-u v\right), \varphi\right\rangle\right| \leq \int_{\Omega}\left|\left(u_{\varepsilon} v_{\varepsilon}-u v\right)(\xi, \eta)^{t} L \varphi(\xi, \eta)\right| d \xi d \eta \\
\leq\left\|\left.\right|^{t} L \varphi\right\|_{q}\left\|u_{\varepsilon} v_{\varepsilon}-u v\right\|_{p} \leq\left\|\left.\right|^{t} L \varphi\right\|_{q}\left\{\left\|v_{\varepsilon}\right\|_{\infty}\left\|u_{\varepsilon}-u\right\|_{p}+\|u\|_{\infty}\left\|v_{\varepsilon}-v\right\|_{p}\right\},
\end{gathered}
$$

onde $1 / p+1 / q=1$. Observe que $u, v \in L^{\infty}(\Omega) \subset L^{p}(\Omega)$ e $\left\|v_{\varepsilon}\right\|_{\infty} \leq\|v\|_{\infty}$. Segue que

$$
L\left(u_{\varepsilon} v_{\varepsilon}\right) \rightarrow L(u v) \text { em } \mathscr{D}^{\prime}(\Omega)
$$

Por (5.37) e (5.38),

$$
L(u v)=f v+u g \mathrm{em} \mathscr{D}^{\prime}(\Omega)
$$

Lema 13. Seja $g \in C(\bar{\Omega})$ tal que $L g \in L^{p}(\Omega), 1 \leq p<\infty$. Então,

$$
L\left(e^{g}\right)=e^{g} \operatorname{Lg} \text { em } \Omega .
$$

Demonstração. Seja $g_{\varepsilon}=\phi_{\varepsilon} * g$. Como $e^{z}$ é uma função Lipschitz no conjunto limitado $\{z \in$ $\left.\mathbb{C} ;|z| \leq 2\|g\|_{\infty}\right\}$ e $\left\|g_{\varepsilon}\right\|_{\infty} \leq\|g\|_{\infty}$ para todo $\varepsilon>0$, existe uma constante $K>0$ tal que

$$
\left\|e^{g_{\varepsilon}}-e^{g}\right\|_{\infty} \leq K\left\|g-g_{\varepsilon}\right\|_{\infty} \text { para todo } \varepsilon>0 .
$$

Assim, temos a seguinte estimativa:

$$
\left\|e^{g} L(g)-e^{g_{\varepsilon}} L\left(g_{\varepsilon}\right)\right\|_{p} \leq\left\|e^{g} L(g)-e^{g_{\varepsilon}} L(g)\right\|_{p}+\left\|e^{g_{\varepsilon}} L(g)-e^{g_{\varepsilon}} L\left(g_{\varepsilon}\right)\right\|_{p}
$$




$$
\begin{aligned}
& \leq\|L(g)\|\left\|_{p}\right\| e^{g}-e^{g_{\varepsilon}}\left\|_{\infty}+\right\| e^{g_{\varepsilon}}\left\|_{\infty}\right\| L(g)-L\left(g_{\varepsilon}\right) \|_{p} . \\
& \leq\|L(g)\|_{p} K\left\|g-g_{\varepsilon}\right\|_{\infty}+e^{\|g\|_{\infty}}\left\|L(g)-L\left(g_{\varepsilon}\right)\right\|_{p} .
\end{aligned}
$$

Logo, pelo Corolário 8 temos que

$$
\left\|L\left(e^{g_{\varepsilon}}\right)-e^{g} L(g)\right\|_{p} \rightarrow 0 \text { quando } \varepsilon \rightarrow 0 .
$$

Segue que

$$
L\left(e^{g_{\varepsilon}}\right) \rightarrow e^{g} L(g) \text { em } \mathscr{D}^{\prime}(\Omega) .
$$

Por outro lado, dado $\varphi \in C_{c}^{1}(\Omega)$,

$$
\begin{gathered}
\left|\left\langle L\left(e^{g_{\varepsilon}}\right)-L\left(e^{g}\right), \varphi\right\rangle\right| \leq\left.\int_{\Omega}\left|\left(e^{g_{\varepsilon}}-e^{g}\right)(\xi, \eta)\right|\right|^{t} L \varphi(\xi, \eta)|d \xi d \eta \leq| \Omega\left|\left\|\left.\right|^{t} L \varphi\right\|_{\infty}\left\|e^{g_{\varepsilon}}-e^{g}\right\|_{\infty}\right. \\
\leq|\Omega|\left\|\left.\right|^{t} L \varphi\right\|_{\infty} K\left\|g_{\varepsilon}-g\right\|_{\infty} .
\end{gathered}
$$

Segue que

$$
L\left(e^{g_{\varepsilon}}\right) \rightarrow L\left(e^{g}\right) \text { em } \mathscr{D}^{\prime}(\Omega) .
$$

Por (5.39) e (5.40)

$$
L\left(e^{g}\right)=e^{g} L(g) \text { em } \mathscr{D}^{\prime}(\Omega) .
$$

Agora, vamos demonstrar um resultado análogo aos Lemas 12 e 13 no caso em que $L$ é o campo em estudo nesse texto. Seja $L$ um campo vetorial complexo definido no aberto $\tilde{\Omega}$ de $\mathbb{R}^{2}$ pertencente a classe $\mathscr{X}(\tilde{\Omega})$. Ressaltamos que o estudo que será feito a seguir só tem relevância quando $L$ não é de classe $C^{1}$, uma vez que o caso de $L$ de classe $C^{1}$ já esta demonstrado acima.

Seja $Z: \tilde{\Omega} \rightarrow \mathbb{C}$ uma integral primeira de $L$, de classe $C^{1+\varepsilon}$, que é um homeomorfismo sobre sua imagem. Sem perda de generalidade, como fizemos na seção anterior, podemos supor

$$
L=H_{Z} \operatorname{em} \tilde{\Omega}
$$

onde $H_{Z}$ é o hamiltoniano de $Z$.

A partir de agora, suponha que $u, v \in C(\bar{\Omega})$ e $f:=L u, g:=L v \in L^{p}(\Omega), \operatorname{com} p>2+\sigma_{\mathrm{M}}$ (onde $\sigma_{\mathrm{M}}>0$ é dado em (5.4), página 72)

O campo $L$ é de classe $C^{1}$ em $\Omega \backslash \Sigma$ (condição (iii), página 71). Segue do Lema 12 que

$$
L(u v)=f v+u g \text { em } \Omega \backslash \Sigma .
$$

Defina

$$
F=f v+u g \text { em } \Omega \text {. }
$$

Note que $F \in L^{p}(\Omega)$, com $p>2+\sigma_{\mathrm{M}}$. Logo, $T_{Z} F \in C(\bar{\Omega})$ (Teorema 6, página 80) e $L\left(T_{Z} F\right)=F$ em $\Omega$ (Teorema 5, página 78 ). Defina

$$
H=u v-T_{Z} F \text { em } \Omega .
$$


Assim, $H \in C(\bar{\Omega})$ e $L H=0$ em $\Omega \backslash \Sigma$. Seja $h: Z(\bar{\Omega}) \rightarrow \mathbb{C}$ dada por

$$
h=H \circ Z^{-1} .
$$

Temos que $h$ é contínua em $Z(\bar{\Omega})$. Além disso, com um argumento análogo ao da demonstração do Teorema 2 (página 53), usando o Teorema de extensão de funções holomorfas (Teorema 23 , página 132), podemos demonstrar que $h$ é holomorfa em $Z(\Omega)$. Segue daí que $H=h \circ Z$ é solução do campo $L$ em $\Omega$, isto é, $L H=0 \mathrm{em} \Omega$.

Logo, temos que

$$
L(u v)=L\left(T_{Z} F+H\right)=L\left(T_{Z} F\right)=f v+u g \operatorname{em} \Omega .
$$

Usando o Lema 13 e um argumento análogo ao acima, é possível demonstrar que se $g \in C(\bar{\Omega})$ e $L g \in L^{p}(\Omega), p>2+\sigma_{\mathrm{M}}$, então

$$
L\left(e^{g}\right)=e^{g} \operatorname{Lg} \text { em } \Omega .
$$

Portanto, fica demonstrado o seguinte lema:

Lema 14. Seja $L$ um campo vetorial complexo definido no aberto $\tilde{\Omega}$ e pertencente a classe $\mathscr{X}(\tilde{\Omega})$ (descrita na página 71 . Veja também o Capítulo 3). Seja $\Omega$ um aberto compactamente contido em $\tilde{\Omega}$ e $\sigma_{\mathrm{M}}>0$ dado por (5.4) (na página 72 ).

1. Se $u, v \in C(\bar{\Omega})$ e $L u, L v \in L^{p}(\Omega), p>2+\sigma_{\mathrm{M}}$, então

$$
L(u v)=L(u) v+u L(v) \text { em } \Omega .
$$

2. Se $g \in C(\bar{\Omega})$ e $L g \in L^{p}(\Omega), p>2+\sigma_{\mathrm{M}}$, então

$$
L\left(e^{g}\right)=e^{g} L(g) \text { em } \Omega
$$

\subsubsection{Princípio da Similaridade para $L$}

Sejam $\tilde{\Omega}$ um aberto de $\mathbb{R}^{2}, \Omega$ um aberto conexo compactamente contido em $\tilde{\Omega}, L$ um campo vetorial complexo definido em $\tilde{\Omega}$ que pertence a classe $\mathscr{X}(\tilde{\Omega})$ (descrita na página 71 . Veja também o Capítulo 3) e $Z: \tilde{\Omega} \rightarrow \mathbb{C}$ uma integral primeira global de $L$, de classe $C^{1+\varepsilon}$, com $Z$ homeomorfismo sobre sua imagem.

O Princípio da Similaridade para algumas classes de campos vetoriais já foi estudado em (MEZIANI, 1999), (BERHANU; HOUNIE; SANTIAGO, 2001) e (MEZIANI, 2005). Como consequência dos teoremas de resolubilidade (Teorema 5, página 85, e 8, página 88), iremos provar o Princípio da Similaridade para o campo $L$.

O teorema a seguir pode ser encontrado em (CAMPANA; SILVA; MEZIANI, 2017). 
Teorema 10. (Princípio da Similaridade para o campo $L$ ) Seja $a, b \in L^{p}(\Omega), p>2+\sigma_{\mathrm{M}}$, $\sigma_{\mathrm{M}}>0$ (dado por (5.4), página 72). Então, para toda $u \in C(\Omega)$ solução da equação

$$
L u=a u+b \bar{u} \text { em } \Omega
$$

existe uma função $h$ holomorfa em $Z(\Omega)$ e uma função $s \in C^{\beta}(\bar{\Omega}), \beta=\left(2-q-\tau_{\mathrm{M}}\right) / q, \tau_{\mathrm{M}}=$ $\sigma_{\mathrm{M}} /\left(\sigma_{\mathrm{M}}+1\right), q=p /(p-1)$, tal que

$$
u(x, y)=h(Z(x, y)) e^{s(x, y)}, \forall(x, y) \in \Omega .
$$

Reciprocamente, para toda função holomorfa $h$ em $Z(\Omega)$ existe $s \in C^{\beta}(\bar{\Omega})$ tal que a função $u$ dada por (5.42) é solução da equação (5.41).

Demonstração. Esta demonstração é uma adaptação da prova do Teorema 4.1 do artigo (MEZIANI, 2005).

Como na seção anterior, podemos supor sem perda de generalidade que $L=H_{Z}$ em $\tilde{\Omega}$.

Suponha que $u \in C(\Omega)$ e que $u$ não é identicamente nula. Como $L$ pertence a classe $\mathscr{X}(\tilde{\Omega})$ segue que $L$ é elítico de classe $C^{1}$ em $\Omega \backslash \Sigma$ e $Z$ é de classe $C^{2}$ em $\Omega \backslash \Sigma$ (lembrando que $\Sigma$ denota o conjunto característico de $L$ ). Assim, pelo Corolário 7 (página 90) a função $u$ possui uma representação da forma (5.42) em uma vizinhança de cada ponto $(x, y) \notin \Sigma$. Logo, $u$ possui zeros isolados em $\Omega \backslash \Sigma$. Defina a função $\phi$ em $\Omega$ por $\phi=\bar{u} / u$ nos pontos onde $u$ é não nula e $\phi=0$ sobre $\Sigma$ e nos pontos onde $u=0$. Note que $\phi \in L^{\infty}(\Omega)$. Segue que $a+b \phi \in L^{p}(\Omega)$, $p>2+\sigma_{\mathrm{M}}$. Considere a equação

$$
L s=-(a+b \phi) \mathrm{em} \Omega
$$

Pelos teoremas 5 (página 78) e 6 (página 80 ) esta equação possui uma solução $s \in C^{\beta}(\bar{\Omega})$, com $\beta=\left(2-q-\tau_{\mathrm{M}}\right) / q, \tau_{\mathrm{M}}=\sigma_{\mathrm{M}} /\left(\sigma_{\mathrm{M}}+1\right)$ e $q=p /(p-1)$. Defina

$$
v=u e^{s} \mathrm{em} \Omega \text {. }
$$

Temos que $v \in C(\bar{\Omega})$ e pelo Lema 14 (página 95), aplicado localmente, temos que

$$
L v=0 \mathrm{em} \Omega .
$$

Então, $v$ pode ser fatorada como

$$
v=h \circ Z \mathrm{em} \Omega
$$

com $h$ holomorfa em $Z(\Omega)$ (Teorema 2, página 53). Isto prova a primeira parte do teorema.

Agora, seja $h$ uma função holomorfa em $Z(\Omega)$. Defina a função $\varphi \operatorname{em} Z(\Omega)$ por $\varphi=\bar{h} / h$ nos pontos onde $h$ não se anula e $\varphi=0$ nos pontos onde $h=0$. Então, $\tilde{\varphi}=\varphi \circ Z \in L^{\infty}(\Omega)$. Consequentemente, $b \tilde{\varphi} \in L^{p}(\Omega), p>2+\sigma_{\mathrm{M}}$. Assim, pelo Teorema 8 (página 88 ) a equação

$$
L s=a+b \tilde{\varphi} e^{\bar{s}-s} \mathrm{em} \Omega,
$$


tem uma solução $s \in C^{\beta}(\bar{\Omega})(0<\beta<1$ como acima). Segue que, $u$ dada por

$$
u(x, y)=h(Z(x, y)) e^{s(x, y)}, \quad(x, y) \in \Omega
$$

é solução de (5.41) em $\Omega$. 



\section{O PROBLEMA DE RIEMANN-HILBERT}

Seja $L$ um campo vetorial complexo definido no aberto $\tilde{\Omega}$ de $\mathbb{R}^{2}$. Seja $\Omega$ um aberto simplesmente conexo limitado, com bordo de classe $C^{1+\varepsilon}, 0<\varepsilon<1$, compactamente contido em $\tilde{\Omega}$, isto é, $\Omega$ é um compacto de $\mathbb{R}^{2}$ e $\bar{\Omega} \subset \tilde{\Omega}$.

Seja $F: \bar{\Omega} \times V \rightarrow \mathbb{C}$, onde $V$ é um aberto de $\mathbb{C}$, uma função. Neste capítulo vamos estudar o seguinte problema de contorno:

$$
\left\{\begin{array}{lll}
L u=F(x, y, u) & \text { em } & \Omega, \\
\Re(\bar{\Lambda} u)=\phi & \text { em } & \partial \Omega,
\end{array}\right.
$$

onde $\Lambda \in C^{\alpha}(\partial \Omega),|\Lambda|=1, \phi \in C^{\alpha}(\partial \Omega ; \mathbb{R}), 0<\alpha<1$.

O Problema de Riemann-Hilbert para a equação $L u=F(x, y, u)$ consiste em encontrar uma solução contínua para o problema de contorno acima. Para $L$ pertencente à classe $\mathscr{X}(\tilde{\Omega}) \mathrm{e}$ $F \in \mathscr{F}_{\Psi}^{\beta} \cup \mathscr{F}$ obtemos soluções Hölder contínuas para o problema. Descreveremos a seguir a classe $\mathscr{X}(\tilde{\Omega})$ (que é a classe central de estudo dessa tese) e as famílias de funções $\mathscr{F}_{\Psi}^{\beta}$ e $\mathscr{F}$.

A classe $\mathscr{X}(\tilde{\Omega})$ foi definida inicialmente na Seção 3.1 (página 47 ) onde foram estudas suas propriedades.

Dizemos que um campo vetorial complexo definido no aberto $\tilde{\Omega}$ de $\mathbb{R}^{2}$ pertence à classe $\mathscr{X}(\tilde{\Omega})$ se $L$ satisfaz as seguintes condições:

(i) $L$ é um campo não singular de classe $C^{\varepsilon}, 0<\varepsilon<1$, em $\tilde{\Omega}$;

(ii) O conjunto característico $\Sigma$ de $L$ é uma subvariedade mergulhada de dimensão 1 de $\Omega$, de classe $C^{1+\varepsilon}$, com um número finito de componentes conexas;

(iii) $L$ é de classe $C^{1}$ em $\tilde{\Omega} \backslash \Sigma$;

(iv) Para cada $\mathrm{p} \in \tilde{\Omega} \backslash \Sigma$, existe uma integral primeira $Z: U \rightarrow \mathbb{C}$ de $L$, de classe $C^{2}$, com $U \subset \tilde{\Omega} \backslash \Sigma$ aberto e $p \in U$, tal que $Z: U \rightarrow Z(U) \subset \mathbb{C}$ é um homeomorfismo; 
(v) Para cada $\mathrm{p} \in \Sigma$, existem uma integral primeira $Z: U \rightarrow \mathbb{C}$ de $L$, com $U \subset \tilde{\Omega}$ uma vizinhança aberta de p, tal que $Z: U \rightarrow Z(U) \subset \mathbb{C}$ é um homeomorfismo que satisfaz $Z_{x y}, Z_{y x} \in C(U)$ e $Z_{x y}=Z_{y x}$ em $U$, e coordenadas locais $(s, t)$ em $U$, de classe $C^{2}$ centradas em $\mathrm{p}$, tal que nas novas variáveis a integral primeira $Z$ se escreve como

$$
Z_{\sigma, \beta}(s, t)=s+i\left(\frac{t|t|^{\sigma}}{\sigma+1}+\beta(s)\right)
$$

onde $\beta$ é uma função a valores reais de classe $C^{2+\varepsilon}$ que depende apenas de $s$ e $\sigma \geq \varepsilon$ é uma constante. Além disso, $Z$ satisfaz a seguinte condição: se $L=A \partial_{x}+B \partial_{y}$ em $\Omega$, então a função $\lambda: U \rightarrow \mathbb{C}$ definida por

$$
\lambda(x, y)= \begin{cases}\frac{A(x, y)}{Z_{y}(x, y)}, & \text { se } Z_{y}(x, y) \neq 0, \\ -\frac{B(x, y)}{Z_{x}(x, y)}, & \text { se } \quad Z_{x}(x, y) \neq 0 .\end{cases}
$$

é de classe $C^{1}$ em $U$.

Seja $L$ um campo vetorial complexo definido no aberto $\tilde{\Omega}$ pertencente a classe $\mathscr{X}(\tilde{\Omega})$. Como no capítulo anterior, seja

$$
\sigma_{\mathrm{M}}=\max _{1 \leq j \leq N}\left\{\sigma_{j}\right\}
$$

onde $\sigma_{1}, \cdots, \sigma_{N}$ são os respectivos tipos de $L$ ao longo das componentes conexas de $\Sigma$, a saber, $\Sigma_{1}, \cdots, \Sigma_{N}$. (Veja a Proposição 10, página 51, e a Observação 21, página 52).

Agora, vamos definir as famílias de funções $\mathscr{F}_{\Psi}^{\beta}$ e $\mathscr{F}$. Recorde que no capítulo anterior estudamos a equação $L u=F(x, y, u)$ para estas famílias de funções.

Seja $\Psi \in L^{p}\left(\bar{\Omega} ; \overline{\mathbb{R}}_{+}\right)$, com $p>2+\sigma_{\mathrm{M}}, \sigma_{\mathrm{M}}>0$ dado em (6.4) e seja $\beta$ uma constante tal que $0<\beta \leq 1$. Definimos $\mathscr{F}_{\Psi}^{\beta}$ como o conjunto das funções $F: \bar{\Omega} \times \mathbb{C} \rightarrow \mathbb{C}$ que satisfazem

- $F(., \zeta) \in L^{p}(\bar{\Omega})$, para todo $\zeta \in \mathbb{C}$

- $\left|F\left(x, y, \zeta_{1}\right)-F\left(x, y, \zeta_{2}\right)\right| \leq \Psi(x, y)\left|\zeta_{1}-\zeta_{2}\right|^{\beta}$, para todos $\zeta_{1}, \zeta_{2} \in \mathbb{C}$.

Chamamos de $\mathscr{F}$ o conjunto das $F$ que têm a forma

$$
F(x, y, \zeta)=g(x, y) Q(x, y, \zeta)+f(x, y)
$$

onde $f, g \in L^{p}(\bar{\Omega}), p>2+\sigma_{\mathrm{M}}$ e $Q: \bar{\Omega} \times \mathbb{C} \rightarrow \mathbb{C}$ é uma função contínua e limitada.

Um caso particular interessante, que estudaremos na Seção 6.5, é quando a equação diferencial do problema de Riemann-Hilbert é do tipo Vekua, isto é, a função $F$ que aparece no problema de contorno (6.1) pertence à família $\mathscr{F}_{\Psi}^{1}$ e é da forma

$$
F(x, y, u)=a(x, y) u+b(x, y) \bar{u}+f(x, y), \operatorname{com} a, b, f \in L^{p}(\Omega), p>2+\sigma_{\mathrm{M}} .
$$


Esta equação tem um comportamento linear na variável $u$, quando consideramos $u$ como elemento do espaço vetorial das funções contínuas em $\bar{\Omega}$ com escalares reais (com escalares complexos a linearidade não ocorre). Este caso será tratado separadamente dos outros casos pelo fato da técnica aplicada a ele ser diferente. Veja a Seção 6.5, página 118.

\subsection{O operador integral $T_{\mathscr{Z}}^{K}$}

Sejam $\tilde{\Omega}, \Omega$ abertos e $L$ um campo vetorial complexo pertencente a classe $\mathscr{X}(\tilde{\Omega})$ como anteriormente. Seja

$$
Z: \tilde{\Omega} \rightarrow \mathbb{C}
$$

uma integral primeira global de $L$ de classe $C^{1+\varepsilon}$ que é um homeomorfismo sobre sua imagem. A existência de $Z$ é dada pela Proposição 7, página 37.

Na Seção 5.1 definimos o operador integral $T_{Z}$, para $f \in L^{1}(\Omega)$, dado por

$$
T_{Z} f(x, y)=\frac{1}{2 \pi i} \int_{\Omega} \frac{f(\xi, \eta)}{Z(\xi, \eta)-Z(x, y)} d \xi d \eta,(x, y) \in \bar{\Omega}
$$

Para facilitar a leitura, vamos listar as boas propriedades do operador $T_{Z}$.

(1) Continuidade: o operador $T_{Z}: L^{p}(\bar{\Omega}) \rightarrow C(\bar{\Omega}), p>2+\sigma_{M}$ (onde $\sigma_{M}>0$ é dado em (5.4), na página 72), é contínuo, mais que isso, valem as seguintes desigualdades: para $f \in L^{p}(\Omega), p>2+\sigma_{\mathrm{M}}$, existe uma constante $M\left(p, \sigma_{1}, \ldots, \sigma_{N}, \Omega\right)>0$ tal que

$$
\left|T_{Z} f(x, y)\right| \leq M\left(p, \sigma_{1}, \ldots, \sigma_{N}, \Omega\right)\|f\|_{p}, \quad \forall(x, y) \in \bar{\Omega}
$$

(Teorema 4 , página 72$)$ e existe uma constante $C\left(p, \sigma_{1}, \ldots, \sigma_{N}, \Omega\right)>0$ tal que

$$
\left|T_{Z} f\left(\mathrm{p}_{1}\right)-T_{Z} f\left(\mathrm{p}_{2}\right)\right| \leq C\left(p, \sigma_{1}, \ldots, \sigma_{N}, \Omega\right)\|f\|_{p}\left|Z\left(\mathrm{p}_{1}\right)-Z\left(\mathrm{p}_{2}\right)\right|^{\beta}, \quad \forall \mathrm{p}_{1}, \mathrm{p}_{2} \in \bar{\Omega}
$$

onde $\beta=\frac{2-q-\tau_{\mathrm{M}}}{q}, \operatorname{com} q=\frac{p}{p-1}$ e $\tau_{\mathrm{M}}=\frac{\sigma_{\mathrm{M}}}{\sigma_{\mathrm{M}}+1}$ (Teorema 6 , página 80 ).

(2) Resolubilidade: Para $f \in L^{1}(\Omega)$ temos $H_{Z}\left(T_{Z} f\right)=f$ em $\Omega$, onde $H_{Z}$ é o Hamiltoniano de $Z$ (Teorema 5, página 78). Como $L=\lambda H_{Z}$, com $\lambda \in C^{1}(\tilde{\Omega})$ e $|\lambda|>0 \mathrm{em} \tilde{\Omega}$, temos $u=T_{Z}(f / \lambda)$ é solução da equação $L u=f$ em $\Omega$ (Corolário 4, página 79).

O operador integral que definiremos a seguir é uma modificação de $T_{Z}$ para tratarmos do estudo de problemas de contorno. Tal modificação não afeta as boas propriedades descritas acima como veremos nos próximos resultados.

A partir de agora, vamos supor que $\partial \Omega$ é transversal a $L$, isto é, se $L=A \partial_{x}+B \partial_{y}$ em $\Omega$, então

$$
i^{*} \omega \neq 0
$$


onde $\omega=B d x-A d y$ (é uma 1-forma ortogonal a $L=A \partial_{x}+B \partial_{y}$ ) e $i: \partial \Omega \rightarrow \tilde{\Omega}$ é a aplicação de inclusão.

Como consequência da transversalidade, $Z(\partial \Omega)$ é uma curva fechada simples de classe $C^{1+\varepsilon}$. Veja a Seção 2.5 (página 44).

Proposição 18. Seja $Z$ como em (6.6). Existe um homeomorfismo de classe $C^{1}, \mathscr{Z}: U \rightarrow W$, onde $U \subset \tilde{\Omega}$ aberto com $\bar{\Omega} \subset U$ e $W$ um aberto de $\mathbb{C}$ com $\overline{\mathbb{D}} \subset W$, tal que $L \mathscr{Z}=0$ em $\Omega$ e $\mathscr{Z}(\bar{\Omega})=\overline{\mathbb{D}}$. Mais do que isso, vale a seguinte fatoração

$$
\mathscr{Z}=h \circ Z \text { em } U
$$

onde $h: Z(U) \rightarrow W$ é um difeomorfismo de classe $C^{1}$ tal que $h$ é holomorfo em $Z(\Omega)$ e $h(Z(\bar{\Omega}))=$ $\overline{\mathbb{D}}$.

Demonstração. Como $\partial \Omega$ é transversal a $L$, temos que $Z(\partial \Omega)$ é uma curva fechada simples de classe $C^{1+\varepsilon}$. Pelo Teorema 21, página 131, existe um difeomorfismo $h: \bar{V} \rightarrow \bar{W} \subset \mathbb{C}$, com $V, W \subset \mathbb{C}$ abertos, $Z(\bar{\Omega}) \subset V$ e $\overline{\mathbb{D}} \subset W$, tal que $h$ é holomorfa em $Z(\Omega)$ e $h(Z(\bar{\Omega}))=\overline{\mathbb{D}}$. Assim, $\mathscr{Z}: U \rightarrow W, U=Z^{-1}(V) \subset \tilde{\Omega}$, dada por $\mathscr{Z}=h \circ Z$ é um homeomorfismo (de classe $C^{1}$ ) com $L \mathscr{Z}=0$ em $\Omega$ e $\mathscr{Z}(\bar{\Omega})=\overline{\mathbb{D}}$.

A função $\mathscr{Z}$, dada pela proposição acima, é uma integral primeira de $L$ em $\Omega$ e mantém as boas propriedades da integral primeira $Z$ relativas as estimativas do tipo que se encontram no item (1), da lista de boas propriedades do operador $T_{Z}$ feita acima, como veremos a seguir.

O próximo resultado é consequência da fatoração descrita na Proposição 18 acima.

Teorema 11. Para $f \in L^{1}(\Omega)$ considere $T_{\mathscr{Z}} f$ definido por

$$
T_{\mathscr{Z}} f(x, y)=\frac{1}{2 \pi i} \int_{\Omega} \frac{f(\xi, \eta)}{\mathscr{Z}(\xi, \eta)-\mathscr{Z}(x, y)} d \xi d \eta,(x, y) \in \bar{\Omega},
$$

sendo $\mathscr{Z}$ dada pela Proposição 18.

(1)' Para $f \in L^{p}(\Omega), p>2+\sigma_{\mathrm{M}}$, existe uma constante $M\left(p, \sigma_{1}, \ldots, \sigma_{N}, \Omega\right)>0$ tal que

$$
\left|T_{\mathscr{Z}} f(x, y)\right| \leq M\left(p, \sigma_{1}, \ldots, \sigma_{N}, \Omega\right)\|f\|_{p}, \quad \forall(x, y) \in \bar{\Omega},
$$

e existe uma constante $C\left(p, \sigma_{1}, \ldots, \sigma_{N}, \Omega\right)>0$ tal que

$$
\begin{aligned}
& \quad\left|T_{\mathscr{Z}} f\left(\mathrm{p}_{1}\right)-T_{\mathscr{Z}} f\left(\mathrm{p}_{2}\right)\right| \leq C\left(p, \sigma_{1}, \ldots, \sigma_{N}, \Omega\right)\|f\|_{p}\left|\mathscr{Z}\left(\mathrm{p}_{1}\right)-\mathscr{Z}\left(\mathrm{p}_{2}\right)\right|^{\beta}, \quad \forall \mathrm{p}_{1}, \mathrm{p}_{2} \in \bar{\Omega}, \\
& \text { sendo } \beta=\frac{2-q-\tau_{\mathrm{M}}}{q}, \operatorname{com} q=\frac{p}{p-1} \text { e } \tau_{\mathrm{M}}=\frac{\sigma_{\mathrm{M}}}{\sigma_{\mathrm{M}}+1} .
\end{aligned}
$$

(2)' Para $f \in L^{1}(\Omega)$, temos $H_{\mathscr{Z}}\left(T_{\mathscr{Z}} f\right)=f$ em $\Omega$, onde $H_{\mathscr{Z}}$ é o Hamiltoniano de $\mathscr{Z}$. 
Demonstração. Usando a notação da Proposição 18 acima podemos escrever

$$
\mathscr{Z}=h \circ Z \text { em } U \text {. }
$$

Seja $K$ a constante de Lipschitz de $h^{-1}$ em $\bar{W}$. Então, para $\mathrm{p}_{1}, \mathrm{p}_{2} \in U$, temos

$$
\left|Z\left(\mathrm{p}_{1}\right)-Z\left(\mathrm{p}_{2}\right)\right| \leq K\left|h\left(Z\left(\mathrm{p}_{1}\right)\right)-h\left(Z\left(\mathrm{p}_{2}\right)\right)\right|=K\left|\mathscr{Z}\left(\mathrm{p}_{1}\right)-\mathscr{Z}\left(\mathrm{p}_{2}\right)\right| .
$$

Daí,

$$
\frac{1}{\left|\mathscr{Z}\left(\mathrm{p}_{1}\right)-\mathscr{Z}\left(\mathrm{p}_{2}\right)\right|} \leq \frac{K}{\left|Z\left(\mathrm{p}_{1}\right)-Z\left(\mathrm{p}_{2}\right)\right|}, \mathrm{p}_{1}, \mathrm{p}_{2} \in U
$$

Aplicando a desigualdade acima podemos generalizar as estimativas de $T_{Z} f$ para $T_{\mathscr{Z}} f$. Isto é, podemos usar o item (1) acima para provar o item (1)'. De fato, seja $f \in L^{p}(\Omega), p>2+\sigma_{\mathrm{M}}$. Usando a desigualdade de Hölder e a desigualdade acima, com $q=p /(p-1)$, temos

$\left|T_{\mathscr{Z}} f(x, y)\right| \leq\|f\|_{p}\left(\int_{\Omega} \frac{d \xi d \eta}{|\mathscr{Z}(\xi, \eta)-\mathscr{Z}(x, y)|^{q}}\right)^{\frac{1}{q}} \leq K^{\frac{1}{q}}\|f\|_{p}\left(\int_{\Omega} \frac{d \xi d \eta}{|Z(\xi, \eta)-Z(x, y)|^{q}}\right)^{\frac{1}{q}} \leq M$, para $(x, y) \in U$, onde $M=M\left(p, \sigma_{1}, \ldots, \sigma_{N}, \Omega\right)>0$ e a última desigualdade são dados pelo item (1) acima (que correspondem ao Teorema 4, página 72 ). Da mesma forma demonstramos a estima Hölder.

O item (2)' segue do item (2) acima (que corresponde ao Teorema 5, página 78) e do fato de $h$ ser holomorfa em $Z(\Omega)$.

Observação 25. Como $L=\lambda H_{\mathscr{Z}}, \operatorname{com} \lambda \in C^{1}(\Omega) \cap C(\bar{\Omega})$ e $|\lambda|>0$ em $\bar{\Omega}$, temos pelo item (2)' do Teorema acima que se $f \in L^{1}(\Omega)$, então $u=T_{\mathscr{Z}}(f / \lambda)$ é solução da equação $L u=f$ em $\Omega$.

Observação 26. As famílias de funções $\mathscr{F}_{\Psi}^{\beta}$ e $\mathscr{F}$ (definidas na página 100) são invariantes por multiplicação por funções de $L^{\infty}(\Omega)$. Isto é, se $F \in \mathscr{F}_{\Psi}^{\beta}$ e $\varphi \in L^{\infty}(\Omega)$, então $\varphi F \in \mathscr{F}_{\Psi}^{\beta}$. O mesmo ocorre com a família $\mathscr{F}$. Assim, sem perda de generalidade, no estudo da equação $L u=F(x, y, u)$ podemos supor que $L=H_{\mathscr{Z}}$ em $\tilde{\Omega}$.

Com base nas Observações 25 e 26 vamos supor daqui em diante que

$$
L=H_{\mathscr{Z}}
$$

em $\tilde{\Omega}$, onde $\mathscr{Z}$ é dada pela Proposição 18.

Seja $\kappa \in \mathbb{Z}^{+}$. Para $f \in L^{p}(\Omega), p>2+\sigma_{\mathrm{M}}$, definimos

$$
\begin{aligned}
T_{\mathscr{Z}}^{\kappa} f(x, y) & =\frac{1}{2 \pi i} \int_{\Omega} \frac{f(\xi, \eta)}{\mathscr{Z}(\xi, \eta)-\mathscr{Z}(x, y)}-\mathscr{Z}^{2 \kappa}(x, y) \frac{1}{2 \pi i} \int_{\Omega} \frac{\mathscr{Z}(x, y) \overline{f(\xi, \eta)}}{1-\mathscr{Z}(x, y) \overline{\mathscr{Z}(\xi, \eta)}} d \xi d \eta, \\
\operatorname{para}(x, y) & \in \bar{\Omega} .
\end{aligned}
$$

Podemos escrever

$$
T_{\mathscr{Z}}^{\kappa} f=T_{\mathscr{Z}} f+H \text { em } \bar{\Omega} .
$$


No próximo teorema mostraremos que $H$ é Hölder contínua em $\bar{\Omega}$ e $L H=0$ em $\Omega$. Assim, concluiremos que $T_{\mathscr{Z}}^{\kappa} f$ tem as mesmas boas propriedades de $T_{\mathscr{Z}} f$.

Como no caso do operador $T_{\mathscr{Z}} f$, o operador $T_{\mathscr{Z}}^{\kappa} f$ é uma generalização de um operador integral clássico relacionado ao operador de Cauchy-Riemann. Veja (BEGEHR, 1994) e (VEKUA, 1962).

Antes de ir para o próximo teorema, vamos provar um pequeno lema.

Lema 15. Sejam $u, v \in \mathbb{C}, \operatorname{com}|u|,|v| \leq 1$ e $|v|>0$. Então

$$
\left|\frac{v}{|v|^{2}}-u\right| \geq|v-u|
$$

Demonstração.

$$
\left|\frac{v}{|v|^{2}}-u\right|^{2}=\left|\frac{v}{|v|^{2}}\right|^{2}+|u|^{2}-2\left\langle\frac{v}{|v|^{2}}, u\right\rangle .
$$

e

$$
|v-u|^{2}=|v|^{2}+|u|^{2}-2\langle v, u\rangle
$$

Então,

$$
\begin{gathered}
\left|\frac{v}{|v|^{2}}-u\right|^{2}-|v-u|^{2}=\left|\frac{v}{|v|^{2}}\right|^{2}-2\left\langle\frac{v}{|v|^{2}}, u\right\rangle-|v|^{2}+2\langle v, u\rangle \\
=\frac{1}{|v|^{2}}-2 \frac{1}{|v|^{2}}\langle v, u\rangle-|v|^{2}+2\langle v, u\rangle=\frac{1}{|v|^{2}}\left\{1-|v|^{4}-2\langle v, u\rangle\left(1-|v|^{2}\right)\right\} \\
=\frac{1}{|v|^{2}}\left\{\left(1+|v|^{2}\right)\left(1-|v|^{2}\right)-2\langle v, u\rangle\left(1-|v|^{2}\right)\right\}=\frac{\left(1-|v|^{2}\right)}{|v|^{2}}\left\{1+|v|^{2}-2\langle v, u\rangle\right\} \\
\geq \frac{\left(1-|v|^{2}\right)}{|v|^{2}}|u-v|^{2} \geq 0 .
\end{gathered}
$$

Isto é,

$$
\left|\frac{v}{|v|^{2}}-u\right|^{2} \geq|v-u|^{2}
$$

Isto implica $\left|\frac{v}{|v|^{2}}-u\right| \geq|v-u|$.

Teorema 12. Seja $f \in L^{p}(\Omega)$, com $p>2+\sigma_{M}$, onde $\sigma_{M}>0$ é dado por (6.4). Então, $L\left(T_{\mathscr{Z}}^{K} f\right)=$ $f$ em $\Omega$ e existem constantes $M=M\left(p, \sigma_{1}, \ldots, \sigma_{N}, \Omega\right)>0$ e $C=C\left(p, \sigma_{1}, \ldots, \sigma_{N}, \Omega\right)>0$ tais que

$$
\left|T_{\mathscr{Z}}^{K} f(x, y)\right| \leq M|| f \|_{p}, \quad \forall(x, y) \in \bar{\Omega}
$$

e

$$
\left|T_{\mathscr{Z}}^{K} f\left(\mathrm{p}_{1}\right)-T_{\mathscr{Z}}^{K} f\left(\mathrm{p}_{2}\right)\right| \leq C|| f \|_{p}\left|\mathscr{Z}\left(\mathrm{p}_{1}\right)-\mathscr{Z}\left(\mathrm{p}_{2}\right)\right|^{\beta}, \quad \forall \mathrm{p}_{1}, \mathrm{p}_{2} \in \bar{\Omega}
$$

onde $0<\beta<1$ é dado por $\beta=\left(2-q-\tau_{\mathrm{M}}\right) / q$, $\operatorname{com} q=p /(p-1)$ e $\tau_{\mathrm{M}}=\sigma_{\mathrm{M}} /\left(\sigma_{\mathrm{M}}+1\right)$. 
Demonstração. Seja $(x, y) \in \bar{\Omega}$. Podemos escrever

$$
T_{\mathscr{Z}}^{\kappa} f(x, y)=T_{\mathscr{Z}} f(x, y)-\mathscr{Z}^{2 \kappa}(x, y) B_{\mathscr{Z}} f(x, y),
$$

onde

$$
B_{\mathscr{Z}} f(x, y) \doteq \frac{1}{2 \pi i} \int_{\Omega} \frac{\mathscr{Z}(x, y) \overline{f(\xi, \eta)}}{1-\mathscr{Z}(x, y) \overline{\mathscr{Z}(\xi, \eta)}} d \xi d \eta .
$$

Como $|\mathscr{Z}(x, y)|<1$ para $(x, y) \in \Omega$ e $L \mathscr{Z}=0$ em $\Omega$, segue que $L\left(B_{\mathscr{Z}} f\right)=0$ em $\Omega$. Assim, como $L\left(T_{\mathscr{Z}} f\right)=f$ em $\Omega$ (Teorema 11, página 102), temos da igualdade (6.12) que $L\left(T_{\mathscr{Z}}^{\mathrm{K}} f\right)=f$ em $\Omega$.

Segue do Teorema 11 (página 102) que, para provar as estimativas (6.10) e (6.11) para $T_{\mathscr{Z}}^{K} f$, é suficiente prová-las para $B_{\mathscr{Z}} f$.

Primeiro, note que para $\left(x_{0}, y_{0}\right) \in \Omega$ tal que $\mathscr{Z}\left(x_{0}, y_{0}\right)=0$ temos $B_{\mathscr{Z}} f\left(x_{0}, y_{0}\right)=0$. Para $(x, y) \in \bar{\Omega} \backslash\left\{\left(x_{0}, y_{0}\right)\right\}$, temos

$$
\left|B_{\mathscr{Z}} f(x, y)\right| \leq \frac{1}{2 \pi} \int_{\Omega} \frac{|f(\xi, \eta)|}{\left|\frac{\mathscr{Z}(x, y)}{|\mathscr{Z}(x, y)|^{2}}-\mathscr{Z}(\xi, \eta)\right|} d \xi d \eta .
$$

Como $|\mathscr{Z}(x, y)|<1$ para $(x, y) \in \bar{\Omega}$, temos, pelo Lema 15 ,

$$
\left|\frac{\mathscr{Z}(x, y)}{|\mathscr{Z}(x, y)|^{2}}-\mathscr{Z}(\xi, \eta)\right| \geq|\mathscr{Z}(x, y)-\mathscr{Z}(\xi, \eta)|,(\xi, \eta) \in \Omega .
$$

Por (6.13) e (6.14) temos

$$
\left|B_{\mathscr{Z}} f(x, y)\right| \leq \frac{1}{2 \pi} \int_{\Omega} \frac{|f(\xi, \eta)|}{|\mathscr{Z}(x, y)-\mathscr{Z}(\xi, \eta)|} d \xi d \eta .
$$

A estimativa (6.10) segue de (6.15) e do Teorema 11.

Sejam $\mathrm{p}_{1}=\left(x_{1}, y_{1}\right), \mathrm{p}_{2}=\left(x_{2}, y_{2}\right) \in \bar{\Omega}$, com $\mathrm{p}_{1} \neq \mathrm{p}_{2}$. Temos

$$
\begin{aligned}
& \left|B_{\mathscr{Z} f}\left(\mathrm{p}_{1}\right)-B_{\mathscr{Z}} f\left(\mathrm{p}_{2}\right)\right| \leq \\
& \quad \frac{\left|\mathscr{Z}\left(\mathrm{p}_{1}\right)-\mathscr{Z}\left(\mathrm{p}_{2}\right)\right|}{2 \pi} \int_{\Omega} \frac{|f(\xi, \eta)|}{\left|1-\mathscr{Z}\left(\mathrm{p}_{1}\right) \overline{\mathscr{Z}(\xi, \eta)}\right|\left|1-\mathscr{Z}\left(\mathrm{p}_{2}\right) \overline{\mathscr{Z}(\xi, \eta)}\right|} d \xi d \eta .
\end{aligned}
$$

Note que, quando $|\mathscr{Z}(\mathrm{p})| \leq 1 / 2$, temos

$$
|1-\mathscr{Z}(\mathrm{p}) \overline{\mathscr{Z}(\xi, \eta)}| \geq 1-\frac{1}{2}|\mathscr{Z}(\xi, \eta)| \geq \frac{1}{2},
$$

para todo $(\xi, \eta) \in \bar{\Omega}$. Assim, no caso em que $\left|\mathscr{Z}\left(\mathrm{p}_{k}\right)\right| \leq \frac{1}{2}$, para $k=1,2$, temos

$$
\int_{\Omega} \frac{|f(\xi, \eta)| d \xi d \eta}{\left|1-\mathscr{Z}\left(\mathrm{p}_{1}\right) \overline{\mathscr{Z}(\xi, \eta)}\right| \mid 1-\mathscr{Z}\left(\mathrm{p}_{2}\right) \overline{\mathscr{Z}(\xi, \eta) \mid}} \leq C(p, \Omega)\|f\|_{p}
$$

para $C(p, \Omega)>0$ constante. 
No caso em que $\left|\mathscr{Z}\left(\mathrm{p}_{1}\right)\right| \leq \frac{1}{2} \leq\left|\mathscr{Z}\left(\mathrm{p}_{2}\right)\right|$ temos

$$
\begin{aligned}
\int_{\Omega} \frac{|f(\xi, \eta)| d \xi d \eta}{\left|1-\mathscr{Z}\left(\mathrm{p}_{1}\right) \overline{\mathscr{Z}(\xi, \eta)}\right| \mid 1-\mathscr{Z}\left(\mathrm{p}_{2}\right) \overline{\mathscr{Z}(\xi, \eta) \mid}} & \leq \frac{2}{\left|\mathscr{Z}\left(\mathrm{p}_{2}\right)\right|} \int_{\Omega} \frac{|f(\xi, \eta)| d \xi d \eta}{\mid \frac{1}{\mathscr{Z}\left(\mathrm{p}_{2}\right)}-\overline{\mathscr{Z}(\xi, \eta) \mid}} \\
& \leq 2^{2} \int_{\Omega} \frac{|f(\xi, \eta)| d \xi d \eta}{\left|\mathscr{Z}\left(\mathrm{p}_{2}\right)-\mathscr{Z}(\xi, \eta)\right|} \\
& \leq M\left(p, \sigma_{1}, \ldots, \sigma_{N}, \Omega\right)|| f \|_{p}
\end{aligned}
$$

e, novamente, a última estimativa é obtida do Teorema 11.

Finalmente, quando $\left|\mathscr{Z}\left(\mathrm{p}_{k}\right)\right| \geq 1 / 2$, para $k=1,2$, temos

$$
\begin{gathered}
\int_{\Omega} \frac{|f(\xi, \eta)| d \xi d \eta}{\left|1-\mathscr{Z}\left(\mathrm{p}_{1}\right) \overline{\mathscr{Z}(\xi, \eta)}\right|\left|1-Z\left(\mathrm{p}_{2}\right) \overline{\mathscr{Z}(\xi, \eta)}\right|} \\
\leq 4 \int_{\Omega} \frac{|f(\xi, \eta)| d \xi d \eta}{\left|\frac{1}{\mathscr{Z}\left(\mathrm{p}_{1}\right)}-\overline{\mathscr{Z}(\xi, \eta)}\right| \mid \frac{1}{\mathscr{Z}\left(\mathrm{p}_{2}\right)}-\overline{\mathscr{Z}(\xi, \eta) \mid}} \\
\leq 4 \int_{\Omega} \frac{|f(\xi, \eta)| d \xi d \eta}{\left|\mathscr{Z}\left(\mathrm{p}_{1}\right)-\mathscr{Z}(\xi, \eta)\right|\left|\mathscr{Z}\left(\mathrm{p}_{2}\right)-\mathscr{Z}(\xi, \eta)\right|} \\
\leq C_{1}\|f\|_{p}\left|\mathscr{Z}\left(x_{1}, y_{1}\right)-\mathscr{Z}\left(x_{2}, y_{2}\right)\right|^{\mu},
\end{gathered}
$$

com $\mu=\left(2-\left(2 q+\tau_{\mathrm{M}}\right)\right) / q$, onde a última desigualdade segue do Teorema 11 .

Segue de (6.16), (6.18), (6.19), e (6.20) que podemos encontrar uma constante positiva $C\left(p, \sigma_{1}, \ldots, \sigma_{N}, \Omega\right)$ tal que

$$
\left|B_{\mathscr{Z}} f\left(x_{1}, y_{1}\right)-B_{\mathscr{Z}} f\left(x_{2}, y_{2}\right)\right| \leq C\left(p, \sigma_{1}, \ldots, \sigma_{N}, \Omega\right)\left|\mathscr{Z}\left(x_{1}, y_{1}\right)-\mathscr{Z}\left(x_{2}, y_{2}\right)\right|^{\frac{2-q-\tau_{\mathrm{M}}}{q}} .
$$

Isto completa a demonstração do teorema.

Proposição 19. Seja $f \in L^{p}(\Omega)$, com $p>2+\sigma_{M}$. Então, $T_{\mathscr{Z}}^{\kappa} f($ dado em (6.9)) satisfaz

$$
\left\{\begin{aligned}
L\left(T_{\mathscr{Z}}^{\kappa} f\right)=f & & \text { em } \Omega \\
\Re\left(\overline{\mathscr{Z} \kappa} T_{\mathscr{Z}}^{\kappa} f\right)=0 & & \text { em } \partial \Omega .
\end{aligned}\right.
$$

Demonstração. Sabemos, pelo Teorema 12, que $L\left(T_{\mathscr{Z}}^{\kappa} f\right)=f$ em $\Omega$. Assim, precisamos verificar somente a segunda condição. Seja $(x, y) \in \bar{\Omega}$. Podemos escrever

$$
T_{\mathscr{Z}}^{\mathcal{K}} f(x, y)=T_{\mathscr{Z}} f(x, y)-\mathscr{Z}^{2 \kappa}(x, y) B_{\mathscr{Z}} f(x, y),
$$

com

$$
B_{\mathscr{Z}} f(x, y)=\frac{1}{2 \pi i} \int_{\Omega} \frac{\mathscr{Z}(x, y) \overline{f(\xi, \eta)}}{1-\mathscr{Z}(x, y) \overline{\mathscr{Z}(\xi, \eta)}} d \xi d \eta
$$


Para $(x, y) \in \partial \Omega$, temos $|\mathscr{Z}(x, y)|=1, \log 01 / \mathscr{Z}(x, y)=\overline{\mathscr{Z}(x, y)}$. Daí obtemos

$$
B_{\mathscr{Z}} f(x, y)=\overline{\frac{1}{2 \pi i} \int_{\Omega} \frac{f(\xi, \eta)}{\mathscr{Z}(\xi, \eta)-\mathscr{Z}(x, y)} d \xi d \eta}=\overline{T_{\mathscr{Z}} f(x, y)},
$$

para $(x, y) \in \partial \Omega$. Por (6.21) e (6.22), temos para $(x, y) \in \partial \Omega$,

$$
\begin{gathered}
\overline{\mathscr{Z}^{\kappa}} T_{\mathscr{Z}}^{\kappa} f(x, y)=\overline{\mathscr{Z}^{\kappa}} T_{\mathscr{Z}} f(x, y)-\mathscr{Z}^{\kappa} B_{\mathscr{Z}} f(x, y) \\
\left.=\overline{\mathscr{Z}^{\kappa}} T_{\mathscr{Z}} f(x, y)-\mathscr{Z}^{\kappa} \overline{T_{\mathscr{Z}} f(x, y)}=2 i \mathfrak{( \overline { Z } \kappa} T_{\mathscr{Z}} f(x, y)\right) .
\end{gathered}
$$

Portanto, $\mathfrak{R}\left(\overline{\mathscr{Z} \kappa} T_{\mathscr{Z}} f(x, y)\right)=0$ para $(x, y) \in \partial \Omega$.

\subsection{0 operador $S_{\mathscr{Z}}$}

Sejam $L$ e $\mathscr{Z}$ como na seção anterior.

Agora vamos estudar o problema de Riemann-Hilbert homogêneo em um caso particular. Para $\varphi \in C(\partial \Omega ; \mathbb{R})$ e $\kappa \in \mathbb{Z}^{+}$, considere o problema

$$
\left\{\begin{aligned}
L(\Phi)=0 & \text { em } \Omega \\
\Re(\overline{\mathscr{Z} \kappa} \Phi)=\varphi & \text { em } \partial \Omega
\end{aligned}\right.
$$

Obtemos as soluções deste problema através do operador de Schwarz, $S_{\mathscr{Z}}$, associado a $L$, que iremos definir agora.

Seja $S_{\mathscr{Z}}: C(\partial \Omega ; \mathbb{R}) \rightarrow C(\bar{\Omega})$ definido por

$$
\left(S_{\mathscr{Z}} \varphi\right)(x, y) \doteq \frac{1}{2 \pi i} \int_{\partial \Omega} \varphi(\xi, \eta) \frac{\mathscr{Z}(\xi, \eta)+\mathscr{Z}(x, y)}{\mathscr{Z}(\xi, \eta)-\mathscr{Z}(x, y)} \frac{d \mathscr{Z}(\xi, \eta)}{\mathscr{Z}(\xi, \eta)}
$$

O leitor familiarizado com a teoria clássica para problemas de contorno para funções holomorfas certamente notou a semelhança com o respectivo operador clássico $S$ (veja o operador de Schwarz clássico (para o disco $\mathbb{D}$ ) em B.4, página 139. Veja também em (VEKUA, 1962) e (BEGEHR, 1994)). Tal semelhança e bom comportamento da integral primeira $\mathscr{Z}$ em $\Omega$ nos permitem generalizar os resultados referentes a $S$ e $\partial / \partial \bar{z}$ para $S_{\mathscr{Z}}$ e $L$.

Proposição 20. Para $\varphi \in C(\partial \Omega ; \mathbb{R})$, a função $S_{\mathscr{Z}} \varphi$ satisfaz

$$
\left\{\begin{aligned}
L\left(S_{\mathscr{Z}} \varphi\right)=0 & \text { em } \Omega \\
\mathfrak{R}\left(S_{\mathscr{Z}} \varphi\right)=\varphi & \operatorname{em} \partial \Omega
\end{aligned}\right.
$$

e $\mathfrak{I}\left(S_{\mathscr{Z}} \varphi\right)\left(x_{0}, y_{0}\right)=0$, onde $\left(x_{0}, y_{0}\right) \in \Omega$ é tal que $\mathscr{Z}\left(x_{0}, y_{0}\right)=0$. Além disso, se $\varphi \in C^{\alpha}(\partial \Omega ; \mathbb{R})$, $0<\alpha<1$, então $S_{\mathscr{Z}} \varphi \in C_{\mathscr{Z}}^{\alpha}(\bar{\Omega}) \subset C^{\alpha}(\bar{\Omega})$.

Demonstração. Usamos o fato de que $\partial \Omega$ é de classe $C^{1}, \mathscr{Z}: \bar{\Omega} \longrightarrow \overline{\mathbb{D}}$ é de classe $C^{1}$ e um homeomorfismo, para aplicar o lema 9 (página 77) para reescrever $S_{\mathscr{Z}} \varphi$ como

$$
\begin{aligned}
\left(S_{\mathscr{Z}} \varphi\right)(x, y) & =\frac{1}{2 \pi i} \int_{\partial \mathbb{D}}\left(\varphi \circ \mathscr{Z}^{-1}\right)(\zeta) \frac{\zeta+\mathscr{Z}(x, y)}{\zeta-\mathscr{Z}(x, y)} \frac{d \zeta}{\zeta} \\
& =\left(S\left(\varphi \circ \mathscr{Z}^{-1}\right)\right)(\mathscr{Z}(x, y)),
\end{aligned}
$$


onde $S$ é o operador de Schwarz clássico em $\mathbb{D}$. Da teoria clássica (veja Proposição 25, página 139), temos que $S\left(\varphi \circ \mathscr{Z}^{-1}\right)$ é uma função holomorfa em $\mathbb{D}, \mathfrak{R}\left(S\left(\varphi \circ \mathscr{Z}^{-1}\right)\right)=\varphi \circ \mathscr{Z}^{-1}$ sobre $\partial \mathbb{D}$, e $\mathfrak{I}\left(S\left(\varphi \circ \mathscr{Z}^{-1}\right)\right)\left(x_{0}, y_{0}\right)=0$. Além disso, $S\left(\varphi \circ \mathscr{Z}^{-1}\right)$ está na classe de funções Hölder contínuas, em $\Omega$, de mesmo expoente de Hölder que $\varphi \circ \mathscr{Z}^{-1}$ em $\partial \Omega$. Note que, de um argumento proveniente do lema 3 (página 44), a função $\varphi \circ \mathscr{Z}^{-1}$ tem o mesmo expoente de Hölder em $\partial \Omega$ que $\varphi$ possui em $\partial \mathbb{D}$. Segue assim que $L\left(S_{\mathscr{Z}} \varphi\right)=0$ em $\Omega, \Re\left(S_{\mathscr{Z}} \varphi\right)=\varphi$ em $\partial \Omega, \mathfrak{I}\left(S_{\mathscr{Z}} \varphi\right)\left(x_{0}, y_{0}\right)=0$ e se $\varphi \in C^{\alpha}(\partial \Omega ; \mathbb{R})$ então $S_{\mathscr{Z}} \varphi \in C_{\mathscr{Z}}^{\alpha}(\bar{\Omega}) \subset C^{\alpha}(\bar{\Omega})$ (veja o Corolário 9 , na página 140 , sobre o caso clássico que é aplicado a $\left.\varphi \circ \mathscr{Z}^{-1}\right)$.

Com um simples cálculo é possível verificar que

$$
\Phi:=\mathscr{Z}^{\kappa} S_{\mathscr{Z}} \varphi
$$

é solução do problema (6.23). A próxima proposição diz que toda solução deste problema é a função $\Phi$, a menos de um polinômio, em $\mathscr{Z}$, com grau no máximo $2 \kappa$.

Proposição 21. Para $\varphi \in C(\partial \Omega ; \mathbb{R})$, a solução geral do problema (6.23) é da forma

$$
\Phi(x, y)=\mathscr{Z}^{\kappa}(x, y)\left(S_{\mathscr{Z}} \varphi\right)(x, y)+\sum_{j=0}^{2 \kappa} b_{j} \mathscr{Z}^{j}(x, y),
$$

onde $b_{j} \in \mathbb{C}$, com $b_{2 \kappa-j}=-\bar{b}_{j}$, para $j=0,1, \ldots, 2 \kappa$. Além disso, se $\varphi \in C^{\alpha}(\partial \Omega ; \mathbb{R}), 0<\alpha<1$, então $\Phi \in C_{\mathscr{Z}}^{\alpha}(\bar{\Omega})$.

Demonstração. Da teoria clássica sobre o estudo do problema de Riemann-Hilbert (veja (BEGEHR, 1994), (GAKHOV, 1966) e (VEKUA, 1962)) temos que a solução geral do problema

$$
\frac{\partial h}{\partial \bar{\zeta}}=0 \quad \text { em } \quad \mathbb{D} \backslash\{0\} \quad \text { e } \quad \Re(h)=\psi \quad \text { sobre } \quad \partial \mathbb{D},
$$

$(\operatorname{com} \psi \in C(\partial \mathbb{D}, \mathbb{R}))$ tendo um polo de no máximo ordem $\kappa$ em $\zeta=0$, tem a forma

$$
h(\zeta)=\frac{1}{2 \pi i} \int_{\partial \mathbb{D}} \psi(\omega) \frac{\omega+\zeta}{\omega-\zeta} \frac{d \omega}{\omega}+i c_{0}+\sum_{j=1}^{\kappa}\left(c_{j} \zeta^{j}-\overline{c_{j}} \zeta^{-j}\right)
$$

$\operatorname{com} c_{j} \in \mathbb{C}, c_{0} \in \mathbb{R}$.

Seja $\Phi$ uma solução do problema (6.23). Seja $H:=\Phi / \mathscr{Z}^{\kappa}$ em $\bar{\Omega} \backslash\left\{\left(x_{0}, y_{0}\right)\right\}$, onde $\left(x_{0}, y_{0}\right)$ é tal que $\mathscr{Z}\left(x_{0}, y_{0}\right)=0$. Como $L \Phi=0$ em $\Omega$, temos, pelo Teorema 2 (página 53), que $\Phi \circ \mathscr{Z}^{-1}$ é uma função holomorfa em $\mathbb{D}$. Assim

$$
H \circ \mathscr{Z}^{-1}(\zeta)=\frac{\Phi \circ \mathscr{Z}^{-1}(\zeta)}{\zeta^{\kappa}}, \zeta \in \mathbb{D},
$$

tem um polo de ordem no máximo $\kappa$ em $0 \mathrm{e}$

$$
\psi:=\mathfrak{R}\left(H \circ \mathscr{Z}^{-1}\right)=\mathfrak{R}\left(\overline{\mathscr{Z}}^{\kappa} \Phi\right) \circ \mathscr{Z}^{-1}=\varphi \circ \mathscr{Z}^{-1} \text { em } \partial \mathbb{D} .
$$


Logo,

$$
H \circ \mathscr{Z}^{-1}(\zeta)=h(\zeta), \zeta \in \mathbb{D}
$$

onde $h$ é dada em (6.27). Assim, $H=h \circ \mathscr{Z}$. Ou seja,

$$
\Phi(x, y)=\mathscr{Z}(x, y)^{\kappa} h(\mathscr{Z}(x, y)),(x, y) \in \bar{\Omega} .
$$

Usando o lema 9 (página 77) para fazer a mudança de variável adequada na integral que faz parte da expressão de $h$, obtemos a forma desejada (6.26). A regularidade Hölder de $\Phi$ é igual à regularidade de $S_{\mathscr{Z}} \varphi$ que é estudada na Proposição 20.

\subsection{O problema de Riemann-Hilbert para equações do tipo $L u=F(x, y, u)$}

Nesta seção vamos estudar o problema de Riemann-Hilbert para equações semilineares. Os resultados desta seção podem ser encontrados em (CAMPANA; SILVA; MEZIANI, 2016). Como na seção anterior, $\tilde{\Omega}$ é uma aberto de $\mathbb{R}^{2}, \Omega$ é um aberto compactamente contido em $\tilde{\Omega}$ e $L$ é um campo vetorial complexo definido em $\Omega$ pertencente a classe $\mathscr{X}(\tilde{\Omega})$. A equação que faz parte do problema abaixo foi estudada na Seção 5.4; o procedimento de estudo é análogo, porém envolve novos elementos, além da troca do operador $T_{Z}$ pelo operador $T_{\mathscr{Z}}^{K}$. Mais especificamente, estudaremos aqui o problema de contorno:

$$
\left\{\begin{array}{lll}
L u=F(x, y, u) & \text { em } & \Omega, \\
\Re(\bar{\Lambda} u)=\phi & \text { em } & \partial \Omega,
\end{array}\right.
$$

onde $F \in \mathscr{F}_{\Psi}^{\beta} \cup \mathscr{F}$, (sendo $\mathscr{F}_{\Psi}^{\beta}$ e $\mathscr{F}$ definidas na Seção 5.4 e que serão descritas a seguir), $\Lambda \in C^{\alpha}(\partial \Omega),|\Lambda|=1$ e $\phi \in C^{\alpha}(\partial \Omega ; \mathbb{R})$, com $0<\alpha<1$.

Para maior conveniência do leitor, iremos definir novamente aqui as famílias de funções $\mathscr{F}_{\Psi}^{\beta}$ e $\mathscr{F}$.

Seja $\Psi \in L^{p}\left(\bar{\Omega} ; \overline{\mathbb{R}}_{+}\right)$, com $p>2+\sigma_{\mathrm{M}}, \sigma_{\mathrm{M}}>0$ dado em (6.4), página 100 , e seja $0<\beta \leq 1$. Definimos $\mathscr{F}_{\Psi}^{\beta}$ como o conjunto das funções $F: \bar{\Omega} \times \mathbb{C} \rightarrow \mathbb{C}$ que satisfazem

- $F(., \zeta) \in L^{p}(\bar{\Omega})$, para todo $\zeta \in \mathbb{C}$

- $\left|F\left(x, y, \zeta_{1}\right)-F\left(x, y, \zeta_{2}\right)\right| \leq \Psi(x, y)\left|\zeta_{1}-\zeta_{2}\right|^{\beta}$, para todos $\zeta_{1}, \zeta_{2} \in \mathbb{C}$

Chamamos de $\mathscr{F}$ o conjunto das $F$ que têm a forma

$$
F(x, y, \zeta)=g(x, y) Q(x, y, \zeta)+f(x, y)
$$

onde $f, g \in L^{p}(\bar{\Omega}), p>2+\sigma_{\mathrm{M}}$ e $Q: \bar{\Omega} \times \mathbb{C} \rightarrow \mathbb{C}$ é uma função contínua limitada. 
Os Teoremas 13 e 14 abaixo tratam do problema de Riemann-Hilbert quando a condição de contorno é mais simples, i.e., quando em (6.28) trocamos $\Lambda$ por $\mathscr{Z}^{\kappa}$, para $\kappa \in \mathbb{Z}^{+}$. Obteremos a versão mais geral no fim da seção, no Teorema 15, através de uma mudança de incógnita.

Teorema 13. Seja $F \in \mathscr{F}_{\Psi}^{\beta}$, com $0<\beta<1$, e sejam $q=p /(p-1)$ e $\tau_{\mathrm{M}}=\sigma_{\mathrm{M}} /\left(\sigma_{\mathrm{M}}+1\right)$, onde $\sigma_{\mathrm{M}}>0$ é dado por (5.4), página 72. Seja $\kappa \geq 0$ um número inteiro. Então, para toda $\varphi \in C^{\alpha}(\partial \Omega ; \mathbb{R})$, com $0<\alpha<1$, o problema de contorno

$$
\left\{\begin{array}{lll}
L u=F(x, y, u) & \text { em } & \Omega \\
\mathfrak{R}(\overline{\mathscr{Z}} u)=\varphi & \text { em } & \partial \Omega
\end{array}\right.
$$

tem uma solução $u \in C_{\mathscr{Z}}^{\alpha_{0}}(\bar{\Omega}) \subset C^{\alpha_{0}}(\bar{\Omega}), \operatorname{com} \alpha_{0}=\min \left\{\alpha, \frac{2-q-\tau_{\mathrm{M}}}{q}\right\}$.

Demonstração. Seja $\Phi=S_{\mathscr{Z}} \varphi$. Lembre que $\Phi \in C_{\mathscr{Z}}^{\alpha}(\bar{\Omega}) \subset C^{\alpha}(\bar{\Omega})$ e satisfaz

$$
\left\{\begin{array}{rcl}
L \Phi=0 & \text { em } \quad & \Omega \\
\Re(\Phi)=\varphi & \text { sobre } & \partial \Omega
\end{array}\right.
$$

(veja a Proposição 20, página 107).

Seja $C(\bar{\Omega})$ o espaço de Banach das funções contínuas em $\bar{\Omega}$, com a norma

$$
\|u\|_{\infty}=\sup \{|u(x, y)| ;(x, y) \in \bar{\Omega}\} .
$$

Para $M_{0}>0$, seja $C_{M_{0}}(\bar{\Omega})$ dado por

$$
C_{M_{0}}(\bar{\Omega})=\left\{u \in C(\bar{\Omega}) ;\|u\|_{\infty} \leq M_{0}\right\}
$$

Como $F \in \mathscr{F}_{\Psi}^{\beta}, 0<\beta<1$, para $M_{0}>0$ suficientemente grande, temos

$$
M\left(p, \sigma_{1}, \ldots, \sigma_{N}, \Omega\right)\left\{\|\Psi\|_{p} M_{0}^{\beta}+\|F(\cdot, 0)\|_{p}\right\}+\|\Phi\|_{\infty} \leq M_{0},
$$

sendo $M\left(p, \sigma_{1}, \ldots, \sigma_{N}, \Omega\right)>0$ a constante que aparece no Teorema 12, página 104 .

Defina o operador $P: C_{M_{0}}(\bar{\Omega}) \rightarrow C_{M_{0}}(\bar{\Omega})$ por

$$
P u(x, y)=T_{\mathscr{Z}}^{\kappa}(F(\cdot, u))(x, y)+\mathscr{Z}^{\kappa} \Phi
$$

onde $T_{\mathscr{Z}}^{K}$ é dado por (6.9), página 103. O operador $P$ é bem definido. De fato, para $F \in \mathscr{F}_{\Psi}^{\beta}$ e $u \in C_{M_{0}}(\bar{\Omega})$ temos

$$
\|F(\cdot, u)\|_{p} \leq\|\Psi\|_{p} M_{0}^{\beta}+\|F(\cdot, 0)\|_{p}
$$

Então, segue de (6.31), (6.32), e do Teorema 12 (página 104), que para toda $u \in C_{M_{0}}(\bar{\Omega})$ temos

$$
|P u(x, y)| \leq M\left(p, \sigma_{1}, \ldots, \sigma_{N}, \Omega\right)\left\{\|\Psi\|_{p} M_{0}^{\beta}+\|F(\cdot, 0)\|_{p}\right\}+\|\Phi\|_{\infty} \leq M_{0},
$$


para todo $(x, y) \in \bar{\Omega}$. Além disso, para $\mathrm{p}_{1}, \mathrm{p}_{2} \in \bar{\Omega}$, temos

$$
\begin{aligned}
\left|P u\left(\mathrm{p}_{1}\right)-P u\left(\mathrm{p}_{2}\right)\right| & \leq\left|T_{\mathscr{Z}}^{\kappa}(F(\cdot, u))\left(\mathrm{p}_{1}\right)-T_{\mathscr{Z}}^{\kappa}(F(\cdot, u))\left(\mathrm{p}_{2}\right)\right|+\left|\mathscr{Z}^{\kappa} \Phi\left(\mathrm{p}_{1}\right)-\mathscr{Z}^{\kappa} \Phi\left(\mathrm{p}_{2}\right)\right| \\
& \leq C\left(p, \sigma_{1}, \ldots, \sigma_{N}, \Omega\right)|| F(\cdot, u)||_{p}\left|\mathscr{Z}\left(\mathrm{p}_{1}\right)-\mathscr{Z}\left(\mathrm{p}_{2}\right)\right|^{\mu}+C_{\mathscr{Z}{ }^{\kappa}}\left|\mathscr{Z}\left(\mathrm{p}_{1}\right)-\mathscr{Z}\left(\mathrm{p}_{2}\right)\right|^{\alpha},
\end{aligned}
$$

onde $C\left(p, \sigma_{1}, \ldots, \sigma_{N}, \Omega\right)>0$ é uma constante que aparece no Teorema 12 (página 104), $\mu=$ $\left(2-q-\tau_{\mathrm{M}}\right) / q$ e $C_{\mathscr{Z}{ }^{\kappa} \Phi}$ é a $\mathscr{Z}$-constante de Hölder de $\mathscr{Z}^{\kappa} \Phi$. Sejam

$$
K_{1}=\sup \left\{\left|\mathscr{Z}\left(\mathrm{p}_{1}\right)-\mathscr{Z}\left(\mathrm{p}_{2}\right)\right|^{\mu-\alpha_{0}} ; \mathrm{p}_{1}, \mathrm{p}_{2} \in \bar{\Omega}\right\}
$$

$\mathrm{e}$

$$
K_{2}=\sup \left\{\left|\mathscr{Z}\left(\mathrm{p}_{1}\right)-\mathscr{Z}\left(\mathrm{p}_{2}\right)\right|^{\alpha-\alpha_{0}} ; \mathrm{p}_{1}, \mathrm{p}_{2} \in \bar{\Omega}\right\},
$$

onde $\alpha_{0}=\min \{\alpha, \mu\}$. Segue da desigualdade acima que

$$
\begin{gathered}
\left|P u\left(\mathrm{p}_{1}\right)-P u\left(\mathrm{p}_{2}\right)\right| \leq\left[\left.C\left(p, \sigma_{1}, \ldots, \sigma_{N}, \Omega\right)|| F(\cdot, u)\right|_{p} K_{1}+C_{\mathscr{Z} \kappa_{\Phi}} K_{2}\right]\left|\mathscr{Z}\left(\mathrm{p}_{1}\right)-\mathscr{Z}\left(\mathrm{p}_{2}\right)\right|^{\alpha_{0}} \\
\leq\left[C\left(p, \sigma_{1}, \ldots, \sigma_{N}, \Omega\right)\left\{|| \Psi \|_{p} M_{0}^{\beta}+|| F(\cdot, 0)||_{p}\right\} K_{1}+C_{\mathscr{Z}{ }^{\kappa} \Phi} K_{2}\right]\left|\mathscr{Z}\left(\mathrm{p}_{1}\right)-\mathscr{Z}\left(\mathrm{p}_{2}\right)\right|^{\alpha_{0}} \\
\leq C_{0}\left|\mathscr{Z}\left(\mathrm{p}_{1}\right)-\mathscr{Z}\left(\mathrm{p}_{2}\right)\right|^{\alpha_{0}},
\end{gathered}
$$

sendo

$$
C_{0}=C\left(p, \sigma_{1}, \ldots, \sigma_{N}, \Omega\right)\left\{\|\Psi\|_{p} M_{0}^{\beta}+\|F(\cdot, 0)\|_{p}\right\} K_{1}+C_{\mathscr{Z} \kappa_{\Phi}} K_{2} .
$$

Como em (5.25), página 86, defina $\Lambda_{M_{0}, C_{0}}=\Lambda_{M_{0}, C_{0}}\left(\mathscr{Z}, \Omega, \alpha_{0}\right)$ como o conjunto das funções $v \in C_{M_{0}}(\bar{\Omega})$ que satisfazem

$$
\left|v\left(x_{1}, y_{1}\right)-v\left(x_{2}, y_{2}\right)\right| \leq C_{0}\left|\mathscr{Z}\left(x_{1}, y_{1}\right)-\mathscr{Z}\left(x_{2}, y_{2}\right)\right|^{\alpha_{0}}, \quad \forall\left(x_{1}, y_{1}\right),\left(x_{2}, y_{2}\right) \in \bar{\Omega} .
$$

Como visto anteriormente, o conjunto $\Lambda_{M_{0}, C_{0}}$ é convexo e compacto em $C(\bar{\Omega})$. Além disso, pelo que foi mostrado acima, $P u \in \Lambda_{M_{0}, C_{0}}$, para toda $u \in C_{M_{0}}(\bar{\Omega})$.

Afirmamos que o operador $P$ é contínuo. De fato, para $u, v \in C_{M_{0}}(\bar{\Omega})$, temos

$$
\begin{aligned}
\|P u-P v\|_{\infty} & \leq\left\|T_{Z}^{K}(F(\cdot, u)-F(\cdot, v))\right\|_{\infty} \\
& \leq M\left(p, \sigma_{1}, \ldots, \sigma_{N}, \Omega\right)\|F(\cdot, u)-F(\cdot, v)\|_{p} \\
& \leq M\left(p, \sigma_{1}, \ldots, \sigma_{N}, \Omega\right)\|\Psi\|_{p}\|u-v\|_{\infty}^{\beta}
\end{aligned}
$$

pelo Teorema 12. Assim, a restrição $P: \Lambda_{M_{0}, C_{0}} \rightarrow \Lambda_{M_{0}, C_{0}}$ é contínua. Pelo Teorema do Ponto Fixo de Schauder (Teorema 26, página 133) o operador $P$ tem um ponto fixo, isto é, existe $u \in \Lambda_{M_{0}, C_{0}}$ tal que $P u=u$. Tal ponto fixo satisfaz

$$
u=T_{\mathscr{Z}}^{\kappa}(F(\cdot, u))+\mathscr{Z}^{\kappa} \Phi \in C_{\mathscr{Z}}^{\alpha_{0}}(\bar{\Omega}) \subset C^{\alpha_{0}}(\bar{\Omega}) .
$$

Como $u \in C_{M_{0}}(\bar{\Omega})$, temos $F(\cdot, u) \in L^{p}(\Omega), p>2+\sigma_{\mathrm{M}}$. Pela Proposição 19, página 106, $L u(x, y)=F(x, y, u(x, y))$, para todo $(x, y) \in \Omega$. 
Para $(x, y) \in \partial \Omega$, temos

$$
\overline{\mathscr{Z}^{\kappa}(x, y)} u(x, y)=\overline{\mathscr{Z} \kappa}(x, y) T_{\mathscr{Z}}^{\kappa}(F(\cdot, u))(x, y)+\Phi(x, y) .
$$

Novamente, pela Proposição 19,

$$
\mathfrak{R}\left(\overline{\mathscr{Z} \kappa}(x, y) T_{\mathscr{Z}}^{\kappa}(F(\cdot, u))(x, y)\right)=0 \text { para }(x, y) \in \partial \Omega .
$$

Por (6.34) e (6.35) o ponto fixo $u$ satisfaz a condição de contorno

$$
\mathfrak{R}(\overline{\mathscr{Z}} u)=\mathfrak{R}(\Phi)=\varphi \text { sobre } \partial \Omega \text {. }
$$

Portanto, o ponto fixo $u$ é uma solução do problema (6.30).

Teorema 14. Seja $F \in \mathscr{F}$, isto é,

$$
F(x, y, \zeta)=g(x, y) Q(x, y, \zeta)+f(x, y)
$$

onde $f, g \in L^{p}(\bar{\Omega}), p>2+\sigma_{\mathrm{M}}$ e $Q: \bar{\Omega} \times \mathbb{C} \rightarrow \mathbb{C}$ é uma função contínua limitada. Se $\kappa \geq 0$ inteiro, então, para $\varphi \in C^{\alpha}(\partial \Omega ; \mathbb{R}), 0<\alpha<1$, o problema

$$
\left\{\begin{array}{lcl}
L u=F(x, y, u) & \text { em } & \Omega, \\
\Re\left(\overline{\mathscr{Z}}^{\kappa} u\right)=\varphi & \text { sobre } & \partial \Omega
\end{array}\right.
$$

tem uma solução $u \in C_{\mathscr{Z}}^{\alpha_{0}}(\bar{\Omega}) \subset C^{\alpha_{0}}(\bar{\Omega}), \operatorname{com} \alpha_{0}=\min \left\{\alpha, \frac{2-q-\tau_{\mathrm{M}}}{q}\right\}$.

Demonstração. Tome $K>0$ tal que $K \geq\|Q\|_{\infty}$ e seja $P: C(\bar{\Omega}) \rightarrow C(\bar{\Omega})$ definido por

$$
P u(x, y)=T_{\mathscr{Z}}^{\kappa}(g Q(\cdot, u)+f)(x, y)+\mathscr{Z}^{\kappa}(x, y) \Phi(x, y),
$$

onde $\Phi=S_{\mathscr{Z}} \varphi$ (com $S_{\mathscr{Z}} \varphi$ definido em (6.24), página 107). Como $g Q(\cdot, u)+f \in L^{p}(\bar{\Omega})$, $p>2+\sigma_{\mathrm{M}}$, segue do Teorema 12 (página 104) que

$$
|P u(x, y)| \leq M\left(p, \sigma_{1}, \ldots, \sigma_{N}, \Omega\right)\left\{\|g\|_{p} K+\|f\|_{p}\right\}+\|\Phi\|_{\infty} \doteq M_{0}
$$

para todo $(x, y) \in \bar{\Omega}$. Além disso, pelo Teorema 12 e pela Proposição 20, para $\mathrm{p}_{1}, \mathrm{p}_{2} \in \bar{\Omega} \mathrm{e}$ $u \in C(\bar{\Omega})$ existe $C\left(p, \sigma_{1}, \ldots, \sigma_{N}, \Omega\right)>0$ tal que $\left|P u\left(\mathrm{p}_{1}\right)-P u\left(\mathrm{p}_{2}\right)\right| \leq C\left(p, \sigma_{1}, \ldots, \sigma_{N}, \Omega\right)\left\{|| g \|_{p} K+|| f||_{p}\right\}\left|\mathscr{Z}\left(\mathrm{p}_{1}\right)-\mathscr{Z}\left(\mathrm{p}_{2}\right)\right|^{\mu}+C_{\mathscr{Z}{ }^{\kappa} \Phi}\left|\mathscr{Z}\left(\mathrm{p}_{1}\right)-\mathscr{Z}\left(\mathrm{p}_{2}\right)\right|^{\alpha}$, onde $\mu=\left(2-q-\tau_{\mathrm{M}}\right) / q$, e $C_{\mathscr{Z}{ }^{\kappa} \Phi}>0$ é a $\mathscr{Z}$-constante de Hölder de $\mathscr{Z}^{\kappa} \Phi$. Sejam

$$
\lambda_{1}=\sup \left\{\left|\mathscr{Z}\left(\mathrm{p}_{1}\right)-\mathscr{Z}\left(\mathrm{p}_{2}\right)\right|^{\mu-\alpha_{0}} ; \mathrm{p}_{1}, \mathrm{p}_{2} \in \bar{\Omega}\right\}
$$

$\mathrm{e}$

$$
\lambda_{2}=\sup \left\{\left|\mathscr{Z}\left(\mathrm{p}_{1}\right)-\mathscr{Z}\left(\mathrm{p}_{2}\right)\right|^{\alpha-\alpha_{0}} ; \mathrm{p}_{1}, \mathrm{p}_{2} \in \bar{\Omega}\right\}
$$


onde $\alpha_{0}=\min \{\alpha, \mu\}$. Segue da desigualdade acima que

$$
\left|P u\left(\mathrm{p}_{1}\right)-P u\left(\mathrm{p}_{2}\right)\right| \leq\left[C\left(p, \sigma_{1}, \ldots, \sigma_{N}, \Omega\right)\left\{\|g\|_{p} K+\|f\|_{p}\right\} \lambda_{1}+C_{\mathscr{Z} \kappa_{\Phi}} \lambda_{2}\right]\left|\mathscr{Z}\left(\mathrm{p}_{1}\right)-\mathscr{Z}\left(\mathrm{p}_{2}\right)\right|^{\alpha_{0}} .
$$

Seja

$$
C_{0}=C\left(p, \sigma_{1}, \ldots, \sigma_{N}, \Omega\right)\left\{\|g\|_{p} K+\|f\|_{p}\right\} \lambda_{1}+C_{\mathscr{Z}{ }^{\kappa} \Phi} \lambda_{2} .
$$

Defina $\Lambda_{M_{0}, C_{0}} \subset C(\bar{\Omega})$ como na demostração do Teorema anterior (Teorema 13). Note que $P u \in \Lambda_{M_{0}, C_{0}}$, para toda $u \in C(\bar{\Omega})$.

A restrição $P: \Lambda_{M_{0}, C_{0}} \rightarrow \Lambda_{M_{0}, C_{0}}$ é contínua. De fato, como $Q$ é uniformemente contínua no conjunto compacto, $U=\bar{\Omega} \times\left\{\zeta \in \mathbb{C} ;|\zeta| \leq M_{0}\right\}$, dado $\varepsilon>0$, existe $\delta>0$ tal que

$$
\left|Q\left(x, y, \zeta_{1}\right)-Q\left(x, y, \zeta_{2}\right)\right|<\frac{\varepsilon}{M\left(p, \sigma_{1}, \ldots \sigma_{N}, \Omega\right)\left\{\|g\|_{p}+1\right\}},
$$

para todo $(x, y) \in \bar{\Omega} \mathrm{e}\left|\zeta_{1}-\zeta_{2}\right|<\delta$. Segue que se $u, v \in \Lambda_{M_{0}, C_{0}}$, com $\|u-v\|_{\infty}<\delta$, temos

$$
\begin{aligned}
|P u(x, y)-P v(x, y)| & \leq\left|T_{\mathscr{Z}}^{\kappa}(g\{Q(\cdot, u)-Q(\cdot, v)\})\right| \\
& \leq M\left(p, \sigma_{1}, \ldots, \sigma_{N}, \Omega\right)\|g\|_{p}\|Q(\cdot, u)-Q(\cdot, v)\|_{\infty} \\
& \leq \frac{M\left(p, \sigma_{1}, \ldots, \sigma_{N}, \Omega\right)\|g\|_{p}}{M\left(p, \sigma_{1}, \ldots, \sigma_{N}, \Omega\right)\left\{\|g\|_{p}+1\right\}} \varepsilon<\varepsilon .
\end{aligned}
$$

Portanto a restrição $P: \Lambda_{M_{0}, C_{0}} \rightarrow \Lambda_{M_{0}, C_{0}}$ é contínua. Pelo Teorema do Ponto Fixo de Schauder (Teorema 26, página 133) o operador $P$ possui um ponto fixo em $\Lambda_{M_{0}, C_{0}}$, isto é, existe $u \in \Lambda_{M_{0}, C_{0}}$ tal que $P u=u$. Tal ponto fixo satisfaz

$$
u=T_{\mathscr{Z}}^{\kappa}(F(\cdot, u))+\mathscr{Z}^{\kappa} \Phi \in C_{\mathscr{Z}}^{\alpha_{0}}(\bar{\Omega}) \subset C^{\alpha_{0}}(\bar{\Omega})
$$

e $L u(x, y)=F(x, y, u(x, y))$, para todo $(x, y) \in \Omega$. De forma análogo a prova do teorema anterior, Teorema 13, mostramos que $u$ satisfaz a condição de contorno. Portanto $u$ é uma solução do problema (6.36).

O próximo teorema trata de uma versão do problema de Riemann-Hilbert que é mais geral do que as que foram vistas nos dois teoremas anteriores, a saber, o problema (6.28). A existência de solução do problema está relacionada com o índice de $\Lambda$ associado a $L$ com respeito a $\partial \Omega$. Se $L$ é expresso por $L=A \partial / \partial x+B \partial / \partial y$ no aberto $\tilde{\Omega}$ (que contém $\bar{\Omega}$ ), então o índice de $\Lambda$ associado a $L$ com respeito a $\partial \Omega$ é dado por

$$
\operatorname{Ind}_{L, \partial \Omega}(\Lambda)=-\operatorname{sgn}\left(\mathfrak{I}(A \bar{B})\left(\mathrm{p}_{0}\right)\right) \operatorname{ind}_{\partial \Omega}(\Lambda)
$$

onde $\mathrm{p}_{0}$ é um ponto qualquer de $\tilde{\Omega} \backslash \Sigma$ e sgn denota a função sinal, isto é, sgn : $\mathbb{R} \backslash\{0\} \rightarrow\{-1,1\}$, $\operatorname{sgn}(x)=1$ se $x>0$ e $\operatorname{sgn}(x)=-1$ se $x<0$. Quando $-\operatorname{sgn}\left(\mathfrak{I}(A \bar{B})\left(\mathrm{p}_{0}\right)\right)>0$, a aplicação $\mathscr{Z}: \bar{\Omega} \rightarrow \overline{\mathbb{D}}$ preserva orientação e quando $-\operatorname{sgn}\left(\mathfrak{I}(A \bar{B})\left(\mathrm{p}_{0}\right)\right)<0$ a aplicação $\mathscr{Z}$ inverte a orientação. O índice de $\Lambda$ com respeito a $\partial \Omega$, ind $\partial \Omega(\Lambda)$, é definido na definição 8 (página 39 ). (Veja mais na Seção 2.4, página 38). 
Teorema 15. Seja $F \in \mathscr{F}_{\Psi}^{\beta} \cup \mathscr{F}$, com $0<\beta<1$, onde $\mathscr{F}_{\Psi}^{\beta}$ e $\mathscr{F}$ são famílias de funções definidas na página 109. Sejam $\Lambda \in C^{\alpha}(\partial \Omega),|\Lambda|=1$ e $\varphi \in C^{\alpha}(\partial \Omega ; \mathbb{R})$, com $0<\alpha<1$. Se $\operatorname{Ind}_{L, \partial \Omega}(\Lambda) \geq 0$, então o problema de Riemann-Hilbert

$$
\left\{\begin{array}{lll}
L u=F(x, y, u) & \text { em } & \Omega, \\
\Re(\bar{\Lambda} u)=\varphi & \text { em } & \partial \Omega
\end{array}\right.
$$

tem uma solução $u \in C_{\mathscr{Z}}^{\alpha_{0}}(\bar{\Omega}) \subset C^{\alpha_{0}}(\bar{\Omega})$, onde $\alpha_{0}=\min \left\{\alpha, \frac{2-q-\tau_{\mathrm{M}}}{q}\right\}$ (como nos Teoremas 13 e 14).

Demonstração. Usaremos uma técnica baseada na teoria clássica do problema Riemann-Hilbert (veja (BEGEHR, 1994), (GAKHOV, 1966) e (VEKUA, 1962)) para trocar a função $\Lambda$ na condição de contorno por $\mathscr{Z}^{\kappa}$ através de uma mudança de incógnita. Para isso, faremos uso do operador de Schwarz $S_{\mathscr{Z}}$ definido em (6.24), página 107.

Seja $\kappa:=\operatorname{Ind}_{L, \partial \Omega}(\Lambda)$ o índice de $\Lambda$ associado a $L$ com respeito a $\partial \Omega$ e seja

$$
g(x, y)=\mathscr{Z}^{-\kappa}(x, y) \Lambda(x, y) \text { em } \partial \Omega .
$$

Temos que $\operatorname{Ind}_{L, \partial \Omega}(g)=0$. Consequentemente, existe uma função $\vartheta: \partial \Omega \rightarrow \mathbb{R}$ Hölder contínua (com mesmo expoente de Hölder que $g$ ) tal que

$$
g(x, y)=e^{i \vartheta(x, y)}, \text { para todo }(x, y) \in \partial \Omega
$$

Para $(x, y) \in \bar{\Omega}$ defina

$$
\gamma(x, y)=\left(S_{\mathscr{Z}} \vartheta\right)(x, y) .
$$

Então, $L \gamma=0$ em $\Omega$ e $\Re(\gamma)=\vartheta$ em $\partial \Omega$ (Proposição 20, página 107). Assim,

$$
e^{-i \gamma}=e^{\mathfrak{I}(\gamma)+i \kappa \arg (\mathscr{Z})-i \arg (\Lambda)}=e^{\mathfrak{I}(\gamma)} \mathscr{Z}^{\kappa} \bar{\Lambda} \operatorname{em} \partial \Omega .
$$

Seja $\tilde{F} \in \mathscr{F}_{\Psi}^{\beta} \cup \mathscr{F}$ dada por

$$
\tilde{F}(x, y, \zeta)=\frac{F\left(x, y, e^{i \gamma(x, y)} \zeta\right)}{e^{i \gamma(x, y)}} .
$$

Segue dos Teoremas 13 e 14 que o problema

$$
\begin{cases}L U=\tilde{F}(x, y, U) & \text { em } \quad \Omega, \\ \mathfrak{R}(\overline{\mathscr{Z} \kappa} U)=e^{\mathfrak{I}(\gamma)} \varphi & \text { em } \quad \partial \Omega,\end{cases}
$$

tem uma solução $U \in C_{\mathscr{Z}}^{\alpha_{0}}(\bar{\Omega}), \operatorname{com} \alpha_{0}=\min \left\{\alpha, \frac{2-q-\tau_{\mathrm{M}}}{q}\right\}$. Defina $u=e^{i \gamma} U$ em $\bar{\Omega}$. Temos

$$
L u(x, y)=e^{i \gamma(x, y)} L U(x, y)=e^{i \gamma(x, y)} \tilde{F}(x, y, U(x, y))=F(x, y, u(x, y)),
$$

para todo $(x, y) \in \Omega$. Além disso, por (6.37) e (6.38),

$$
\mathfrak{R}(\bar{\Lambda} u)=e^{-\mathfrak{I}(\gamma)} \mathfrak{R}\left(\overline{\mathscr{Z}}^{\kappa} u\right)=\varphi \operatorname{em} \partial \Omega .
$$

Isto completa a prova do teorema. 


\subsection{Uma propriedade do operador $T_{\mathscr{Z}}$}

Na próxima seção vamos usar uma propriedade técnica do operador integral $T_{\mathscr{Z}}$ que provaremos nessa seção (Proposição 22). Os resultados dessa seção podem ser encontrados em (CAMPANA; MEZIANI, 2016).

Seja $L$ um campo vetorial complexo definido no conjunto aberto $\tilde{\Omega} \subset \mathbb{R}^{2}$ pertencente a classe $\mathscr{X}(\tilde{\Omega})$ e seja $Z: \tilde{\Omega} \rightarrow Z(\tilde{\Omega}) \subset \mathbb{C}$ uma integral primeira global de classe $C^{1+\varepsilon}$ que também é um homeomorfismo. Seja $\Omega$ um aberto simplesmente conexo, com bordo de classe $C^{1+\varepsilon}$ transversal a $L$ e compactamente contido em $\tilde{\Omega}$, isto é, $\bar{\Omega}$ é um compacto de $\mathbb{R}^{2}$ e $\bar{\Omega} \subset \tilde{\Omega}$. Pela Proposição 18 (página 102), reduzindo $\Omega \tilde{\Omega}$, podemos obter uma função $\mathscr{Z}: \tilde{\Omega} \rightarrow \mathbb{C}$, de classe $C^{1}$, tal que $\mathscr{Z}: \tilde{\Omega} \rightarrow \mathscr{Z}(\tilde{\Omega})$ é um homeomorfismo, $\mathscr{Z}$ é uma integral primeira de $L$ em $\Omega$, $\mathscr{Z}(\bar{\Omega})=\overline{\mathbb{D}}$ e $\mathscr{Z}$ mantém as boas propriedades de $Z$ de modo que vale o Teorema 11 (página 102). Sem perda de generalidade podemos supor que

$$
L=H_{\mathscr{Z}} \operatorname{em} \tilde{\Omega} .
$$

Veja as Observações 25 e 26 (página 103).

Proposição 22. Seja $f \in L^{p}(\Omega), p>2+\sigma_{\mathrm{M}}$. Então, para $(s, t) \in \Omega$, temos

$$
\int_{\partial \Omega} \frac{T_{\mathscr{Z}} f(x, y)}{\mathscr{Z}(x, y)-\mathscr{Z}(s, t)} d \mathscr{Z}(x, y)=0 .
$$

O restante desta seção é dedicado à prova desta proposição.

Sejam $1<R_{1}<R_{2}$ tais que $\overline{B_{R_{2}}(0)} \subset \mathscr{Z}(\tilde{\Omega})$ e sejam $V_{1}, V_{2} \subset \tilde{\Omega}$ dados por

$$
V_{j}=Z^{-1}\left(B_{R_{j}}(0)\right) \quad j=1,2 .
$$

Temos então

$$
\bar{\Omega} \subset V_{1} \subset \overline{V_{1}} \subset V_{2} \subset \overline{V_{2}} \subset \tilde{\Omega} .
$$

Considere $\chi:[0, \infty) \rightarrow[0,1]$, uma função $C^{\infty}$, satisfazendo

1. $\chi=1 \mathrm{em}\left[0, R_{1}\right]$,

2. $\chi=0$ em $\left[R_{2}, \infty\right) \mathrm{e}$

3. $\chi$ é estritamente decrescente em $\left[R_{1}, R_{2}\right]$.

Seja $\mathscr{Z}_{\circ}: V_{2} \rightarrow \mathbb{C}$ a função de classe $C^{1}$ dada por

$$
\mathscr{Z}_{\circ}(\mathrm{p})=\frac{\mathscr{Z}(\mathrm{p})}{\chi(|\mathscr{Z}(\mathrm{p})|)} .
$$

Lema 16. A função $\mathscr{Z}_{\circ}: V_{2} \rightarrow \mathbb{C}$ é um homeomorfismo. 
Demonstração. Primeiro vamos mostrar que $\mathscr{Z}_{\circ}$ é uma bijeção. Note que, como $\chi\left(R_{2}\right)=0 \mathrm{e}$ $V_{2}=\mathscr{Z}^{-1}\left(B_{R_{2}}(0)\right)$, temos $\mathscr{Z}_{\circ}\left(V_{2}\right)=\mathbb{C}$.

Sejam $\mathrm{p}_{1}, \mathrm{p}_{2} \in V_{2}$. Se $\left|\mathscr{Z}\left(\mathrm{p}_{1}\right)\right|<\left|\mathscr{Z}\left(\mathrm{p}_{2}\right)\right|$, então $\chi\left(\left|\mathscr{Z}\left(\mathrm{p}_{1}\right)\right|\right) \geq \chi\left(\left|\mathscr{Z}\left(\mathrm{p}_{2}\right)\right|\right)$ e

$$
\left|\mathscr{Z}_{\circ}\left(\mathrm{p}_{1}\right)\right|=\frac{\left|\mathscr{Z}\left(\mathrm{p}_{1}\right)\right|}{\chi\left(\left|\mathscr{Z}\left(\mathrm{p}_{1}\right)\right|\right)}<\frac{\left|\mathscr{Z}\left(\mathrm{p}_{2}\right)\right|}{\chi\left(\left|\mathscr{Z}\left(\mathrm{p}_{2}\right)\right|\right)}=\left|\mathscr{Z}_{\circ}\left(\mathrm{p}_{2}\right)\right| .
$$

Assim, para termos $\mathscr{Z}_{\circ}\left(\mathrm{p}_{1}\right)=\mathscr{Z}_{\circ}\left(\mathrm{p}_{2}\right)$, precisamos ter $\left|\mathscr{Z}\left(\mathrm{p}_{1}\right)\right|=\left|\mathscr{Z}\left(\mathrm{p}_{2}\right)\right|$. Suponha $\mathscr{Z}_{\circ}\left(\mathrm{p}_{1}\right)=$ $\mathscr{Z}_{\circ}\left(\mathrm{p}_{2}\right)$. Pelo que vimos acima, $\left|\mathscr{Z}\left(\mathrm{p}_{1}\right)\right|=\left|\mathscr{Z}\left(\mathrm{p}_{2}\right)\right|$. Olhando para a expressão de $\mathscr{Z}_{\circ}$ em (6.39) concluímos que $\mathscr{Z}\left(\mathrm{p}_{1}\right)=\mathscr{Z}\left(\mathrm{p}_{2}\right)$. Logo, $\mathrm{p}_{1}=\mathrm{p}_{2}$, pois $\mathscr{Z}$ é um homeomorfismo. Portanto, $\mathscr{Z}_{\circ}: V_{2} \rightarrow \mathbb{C}$ é uma bijeção.

Finalmente, vamos provar que $F=\mathscr{Z}_{o}^{-1}$ é contínua. Sejam $\zeta_{0} \in \mathbb{C}$ e $\left(\zeta_{j}\right)_{j \in \mathbb{N}} \subset \mathbb{C}$ tais que $\zeta_{j} \rightarrow \zeta_{0}$. Então a sequência $\left(F\left(\zeta_{j}\right)\right)_{j \in \mathbb{N}}$ tem um ponto de acumulação em $\overline{V_{2}}$. Seja p $\in \overline{V_{2}}$ tal ponto de acumulação. Então, existe uma subsequência $w_{k}:=F\left(\zeta_{j_{k}}\right)$ que converge para p. Temos que $p \in V_{2}$. De fato, suponha que $p \in \partial V_{2}$, então,

$$
\infty=\lim _{k \rightarrow \infty} \mathscr{Z}_{\circ}\left(w_{k}\right)=\lim _{k \rightarrow \infty} \zeta_{j_{k}}=\zeta_{0}
$$

o que é uma contradição. Como $\mathscr{Z}_{\circ}$ é contínua no ponto $\mathrm{p}$, temos $\zeta_{0}=\mathscr{Z}_{\circ}(\mathrm{p})$ e $F\left(\zeta_{0}\right)=\mathrm{p}$. Isto implica que $F\left(\zeta_{j}\right) \rightarrow F\left(\zeta_{0}\right)$ e assim concluímos que $F$ é contínua.

Seja $\left\{\Omega_{n}\right\}_{n \in \mathbb{N}}$ uma sequência de subconjuntos abertos de $\Omega$ tais que $\bar{\Omega}_{n} \subset \Omega_{n+1} \subset$ $\bar{\Omega}_{n+1} \subset \Omega$, para todo $n \in \mathbb{N}$ e $\Omega_{n} \rightarrow \Omega$ quando $n \rightarrow \infty$.

Lema 17. Seja $f \in L^{p}(\Omega), p>2+\sigma_{M}$ e considere

$$
T_{\mathscr{Z}_{\circ}, n} f(x, y):=\frac{1}{2 \pi i} \int_{\Omega_{n}} \frac{f(\xi, \eta) d \xi d \eta}{\mathscr{Z}_{\circ}(\xi, \eta)-\mathscr{Z}_{\circ}(x, y)},(x, y) \in V_{2}
$$

Então $T_{\mathscr{Z}_{\circ}, n} f(x, y) \rightarrow 0$ (uniformemente) quando $(x, y) \rightarrow \partial V_{2}$.

Demonstração. Como $\left|\mathscr{Z}_{\circ}(x, y)\right| \geq\left|\mathscr{Z}_{\circ}(\xi, \eta)\right|$ quando $(x, y) \in V_{2} \backslash \bar{\Omega} \mathrm{e}(\xi, \eta) \in \Omega$, temos

$$
\left|\mathscr{Z}_{\circ}(x, y)\right|-\left|\mathscr{Z}_{\circ}(\xi, \eta)\right| \geq\left|\mathscr{Z}_{\circ}(x, y)\right|-1 \geq 0 .
$$

Daí, para $(x, y) \in V_{2} \backslash \bar{\Omega}$, temos

$$
\begin{gathered}
\left|T_{\mathscr{Z}_{\circ}, n} f(x, y)\right| \leq \frac{1}{2 \pi} \int_{\Omega_{n}} \frac{|f(\xi, \eta)| d \xi d \eta}{\left|\mathscr{Z}_{\circ}(x, y)\right|-1} \\
\leq \frac{1}{2 \pi} \int_{\Omega} \frac{|f(\xi, \eta)| d \xi d \eta}{\left|\mathscr{Z}_{\circ}(x, y)\right|-1}=\frac{1}{2 \pi} \frac{\|f\|_{1}}{\left|\mathscr{Z}_{\circ}(x, y)\right|-1} .
\end{gathered}
$$

A conclusão do lema segue da estimativa acima. 
Considere o campo vetorial complexo

$$
L_{\circ}=\frac{\partial \mathscr{Z}_{\circ}}{\partial y} \partial_{x}-\frac{\partial \mathscr{Z}_{\circ}}{\partial x} \partial_{y} .
$$

Note que, como $\mathscr{Z}_{\circ}$ é uma integral primeira global, de classe $C^{1}$, de $L_{\circ}$ e também um homeomorfismo sobre sua imagem, temos que $L_{\circ}$ é um campo $C^{1}$-hipocomplexo em $V_{2}$. Como $T_{\mathscr{Z}_{o}, n} f \in C^{1}\left(V_{2} \backslash \bar{\Omega}_{n}\right)$ e $L_{\circ}\left(T_{\mathscr{Z}_{o}, n} f\right)=0$ em $V_{2} \backslash \bar{\Omega}_{n}$ temos pela Proposição 6 (página 36) que

$$
T_{\mathscr{Z}_{0}, n} f(x, y)=h_{n}\left(\mathscr{Z}_{\circ}(x, y)\right), \quad(x, y) \in V_{2} \backslash \bar{\Omega}_{n},
$$

onde $h_{n}: \mathscr{Z}_{\circ}\left(V_{2} \backslash \bar{\Omega}_{n}\right) \rightarrow \mathbb{C}$ é uma função holomorfa.

Para $\varepsilon>0$ dado, seja $\delta>0$ (lema 17) tal que

$$
\operatorname{dist}\left((x, y), \partial V_{2}\right)<\delta \Longrightarrow\left|T_{\mathscr{Z}_{0}, n} f(x, y)\right|<\varepsilon
$$

Seja $R \in\left[R_{1}, R_{2}\right]$ tal que $(x, y) \in \mathscr{Z}_{\circ}^{-1}\left(\partial B_{R}(0)\right)$, implica $\operatorname{dist}\left((x, y), \partial V_{2}\right)<\delta$. Segue de $(6.40)$ que,

$$
\left|h_{n}(\zeta)\right|<\varepsilon, \quad \forall \zeta \in \partial B_{R}(0)
$$

$\operatorname{Se}(x, y) \in \Omega$, temos

$$
\begin{aligned}
\int_{\partial \Omega} \frac{T_{\mathscr{Z}_{\circ}, n} f(\xi, \eta) d Z_{\circ}(\xi, \eta)}{\mathscr{Z}_{\circ}(\xi, \eta)-\mathscr{Z}_{\circ}(x, y)} & =\int_{\partial \Omega} \frac{h_{n}\left(\mathscr{Z}_{\circ}(\xi, \eta)\right) d \mathscr{Z}_{\circ}(\xi, \eta)}{\mathscr{Z}_{\circ}(\xi, \eta)-\mathscr{Z}_{\circ}(x, y)} \\
& =\int_{\partial \mathbb{D}} \frac{h_{n}(\zeta) d \zeta}{\zeta-\mathscr{Z}_{\circ}(x, y)} \\
& =\int_{\partial B_{R}(0)} \frac{h_{n}(\zeta) d \zeta}{\zeta-\mathscr{Z}_{\circ}(x, y)},
\end{aligned}
$$

pelo Teorema de Cauchy. Estimando a última integral, obtemos

$$
\begin{aligned}
\left|\int_{\partial B_{R}(0)} \frac{h_{n}(\zeta) d \zeta}{\zeta-\mathscr{Z}_{\circ}(x, y)}\right| & \leq \int_{\partial B_{R}(0)} \frac{\left|h_{n}(\zeta)\right||d \zeta|}{\left|\zeta-\mathscr{Z}_{\circ}(x, y)\right|} \\
& \leq \int_{\partial B_{R}(0)} \frac{\varepsilon|d \zeta|}{R-1} \leq \frac{2 \pi R \varepsilon}{R-1} \leq \frac{2 \pi R_{1} \varepsilon}{R_{1}-1} .
\end{aligned}
$$

Segue de (6.41) e (6.42) que

$$
\int_{\partial \Omega} \frac{T_{\mathscr{Z}_{\circ}, n} f(\xi, \eta) d \mathscr{Z}_{\circ}(\xi, \eta)}{\mathscr{Z}_{\circ}(\xi, \eta)-\mathscr{Z}_{\circ}(x, y)}=0, \quad \forall(x, y) \in \Omega .
$$

Considere as funções $g_{n}$ e $g$ definidas em $\overline{\mathbb{D}}$ por

$$
g_{n}(\zeta)=T_{\mathscr{Z}_{\circ}, n} f\left(\mathscr{Z}_{\circ}^{-1}(\zeta)\right) \text { e } g(\zeta)=T_{\mathscr{Z}_{\circ}} f\left(\mathscr{Z}_{\circ}^{-1}(\zeta)\right)
$$


Note que, como $\sup _{\Omega}\left|T_{\mathscr{Z}_{\circ}, n} f-T_{\mathscr{Z}_{\odot}} f\right| \rightarrow 0$ quando $n \rightarrow \infty$, temos $\sup _{\overline{\mathbb{D}}}\left|g_{n}-g\right| \rightarrow 0$ quando $n \rightarrow \infty$.

Seja $\left(x_{0}, y_{0}\right) \in \Omega, z_{0}=\mathscr{Z}\left(x_{0}, y_{0}\right)$ e seja $r=\operatorname{dist}\left(\partial \mathbb{D}, z_{0}\right)$. Usando (6.43) obtemos a estimativa

$$
\begin{aligned}
\left|\int_{\partial \Omega} \frac{T_{\mathscr{Z}_{\circ}} f(\xi, \eta) d \mathscr{Z}_{\circ}(\xi, \eta)}{\mathscr{Z}_{\circ}(\xi, \eta)-\mathscr{Z}_{\circ}\left(x_{0}, y_{0}\right)}\right| & =\left|\int_{\partial \Omega} \frac{\left\{T_{\mathscr{Z}_{\circ}} f(\xi, \eta)-T_{\mathscr{Z}_{\circ}, n} f(\xi, \eta)\right\} d \mathscr{Z}_{\circ}(\xi, \eta)}{\mathscr{Z}_{\circ}(\xi, \eta)-\mathscr{Z}_{\circ}\left(x_{0}, y_{0}\right)}\right| \\
& =\left|\int_{\partial \mathbb{D}} \frac{\left\{g(\zeta)-g_{n}(\zeta)\right\} d \zeta}{\zeta-z_{0}}\right| \leq \frac{2 \pi}{r}\left\|g_{n}-g\right\|_{\overline{\mathbb{D}}} .
\end{aligned}
$$

Isto implica que, para cada $\left(x_{0}, y_{0}\right) \in \Omega$,

$$
\int_{\partial \Omega} \frac{T_{\mathscr{Z}_{\circ}} f(\xi, \eta) d \mathscr{Z}_{\circ}(\xi, \eta)}{\mathscr{Z}_{\circ}(\xi, \eta)-\mathscr{Z}_{\circ}\left(x_{0}, y_{0}\right)}=0
$$

Finalmente, como $\mathscr{Z}_{\circ}=\mathscr{Z}$ em $V_{1}$ e $\Omega \subset V_{1}$, temos

$$
\int_{\partial \Omega} \frac{T_{\mathscr{Z}} f(x, y)}{\mathscr{Z}(x, y)-\mathscr{Z}(s, t)} d \mathscr{Z}(x, y)=\int_{\partial \Omega} \frac{T_{\mathscr{Z}} f(x, y)}{\mathscr{Z}_{\circ}(x, y)-\mathscr{Z}_{\circ}(s, t)} d \mathscr{Z}_{\circ}(x, y)=0
$$

para $(s, t) \in \Omega$. Isto completa a prova da proposição.

\subsection{O problema de Riemann-Hilbert para a equação $L u=$ $a u+b \bar{u}+f$}

Nesta seção, vamos considerar um tipo de problema de Riemann-Hilbert para o campo $L$ em um aberto simplesmente conexo limitado. Vamos assumir, como nas seções anteriores, que $L$ é um campo vetorial complexo definido na aberto conexo $\tilde{\Omega}$ de $\mathbb{R}^{2}$ pertencente a classe $\mathscr{X}(\tilde{\Omega})$. Seja $\Omega$ um aberto simplesmente conexo compactamente contido em $\Omega$ com bordo, $\partial \Omega$, de classe $C^{1+\varepsilon}$ e transversal a $L$.

Os resultados dessa seção podem ser encontrados em (CAMPANA; MEZIANI, 2016).

Para tal campo vetorial complexo, vamos considerar o seguinte problema de RiemannHilbert

$$
\left\{\begin{array}{lll}
L u=a u+b \bar{u}+f & \text { em } & \Omega, \\
\Re(\bar{\Lambda} u)=\varphi & \text { em } & \partial \Omega,
\end{array}\right.
$$

onde $\Lambda \in C^{\alpha}(\partial \Omega),|\Lambda|=1, \varphi \in C^{\alpha}(\partial \Omega ; \mathbb{R}), 0<\alpha<1$, e $a, b, f \in L^{p}(\bar{\Omega}), p>2+\sigma_{\mathrm{M}}$, onde $\sigma_{\mathrm{M}}>0$ é dado por (6.4), página 100.

A técnica desenvolvida aqui nesta seção é uma generalização de uma técnica da teoria das funções analíticas generalizadas, que pode ser encontrada em (VEKUA, 1962), que em poucas palavras, é utilizada para resolver o problema de Riemann-Hilbert acima quando tomamos no lugar de $L$ o operador de Cauchy-Riemann. 
Pela Proposição 18 (página 102), reduzindo $\tilde{\Omega}$, podemos obter uma função $\mathscr{Z}: \tilde{\Omega} \rightarrow \mathbb{C}$, de classe $C^{1}$, tal que $\mathscr{Z}: \tilde{\Omega} \rightarrow \mathscr{Z}(\tilde{\Omega})$ é um homeomorfismo, $\mathscr{Z}$ é uma integral primeira de $L$ em $\Omega, \mathscr{Z}(\bar{\Omega})=\overline{\mathbb{D}}$ e $\mathscr{Z}$ mantém as boas propriedades de $Z$ de modo que vale o Teorema 11 (página 102). Sem perda de generalidade podemos supor que

$$
L=H_{\mathscr{Z}} \operatorname{em} \tilde{\Omega}
$$

onde $H_{\mathscr{Z}}=\mathscr{Z}_{y} \partial / \partial_{x}-\mathscr{Z}_{x} \partial / \partial_{y}$. Veja as Observações 25 e 26 (página 103).

Para resolver esse problema, antes vamos tratar de uma versão particular do problema que terá a resolubilidade estabelecida usando o operador $T_{\mathscr{Z}}^{\kappa}$ (veja em (6.9), página 103) e o Teorema da Alternativa de Fredholm (Teorema 27, página 133).

Para $\kappa \geq 0$ inteiro, considere o seguinte problema

$$
\left\{\begin{array}{lll}
L u=a u+b \bar{u}+f & \text { em } & \Omega \\
\Re(\overline{\mathscr{Z} \kappa} u)=\varphi & \text { em } & \partial \Omega
\end{array}\right.
$$

onde $a, b, f$ e $\varphi$ como acima.

Note que $u \in C(\bar{\Omega})$ é uma solução de (6.44) se, e somente se, $u$ satisfaz a equação integral

$$
u=T_{\mathscr{Z}}^{\kappa}(a u+b \bar{u})+T_{\mathscr{Z}}^{\kappa}(f)+\Phi
$$

onde $L \Phi=0$ em $\Omega$ e $\Re(\overline{\mathscr{Z} \kappa} \Phi)=\varphi$ sobre $\partial \Omega$.

Podemos reescrever a equação (6.45) como

$$
u=G u+\phi
$$

onde $\phi=T_{\mathscr{Z}}^{\kappa}(f)+\Phi$ e com $G$ sendo o operador integral

$$
G u=T_{\mathscr{Z}}^{K}(a u+b \bar{u}),
$$

definido no espaço de Banach real ${ }^{1}$

$$
X:=\left\{C(\bar{\Omega} ; \mathbb{C}),\|\cdot\|_{\infty}\right\}
$$

É fácil ver que $G$ é um operador linear em $X$.

Afim de aplicar o Teorema de Fredholm (Teorema 27, página 133), vamos estudar a compacidade do operador $G$ e o núcleo do operador $I-G$ nas próximas proposições.

Seja $\mathscr{B}_{X}=\left\{w \in X ;\|w\|_{\infty} \leq 1\right\}$ a bola unitária de $X$.

Proposição 23. O operador $G: X \rightarrow X$ é compacto.

1 Estamos trabalhando aqui com o espaço vetorial das funções contínuas $w: \bar{\Omega} \rightarrow \mathbb{C}$ com escalares reais. 
Demonstração. Para $u \in \mathscr{B}_{X}$ temos pelo Teorema 12 (página 104), desigualdade (6.10), que existe uma constante $M=M\left(p, \sigma_{1}, \ldots, \sigma_{N}, \Omega\right)>0$ tal que

$$
\|G u\|_{\infty} \leq M\left(\|a\|_{p}+\|b\|_{p}\right)\|u\|_{\infty} \leq M\left(\|a\|_{p}+\|b\|_{p}\right)=: M_{0} .
$$

Para $\mathrm{p}_{1}, \mathrm{p}_{2} \in \bar{\Omega}$, temos, pelo Teorema 12, desigualdade (6.11), que existe uma constante $C=$ $C\left(p, \sigma_{1}, \ldots, \sigma_{N}, \Omega\right)>0$ tal que

$$
\begin{aligned}
\left|G u\left(\mathrm{p}_{1}\right)-G u\left(\mathrm{p}_{2}\right)\right| & \leq C|| a u+b \bar{u} \|_{p}\left|\mathscr{Z}\left(\mathrm{p}_{1}\right)-\mathscr{Z}\left(\mathrm{p}_{2}\right)\right|^{\beta} \\
& \leq C\left\{\|a\|_{p}+\|b\| \|_{p}\right\}\|u\|_{\infty}\left|\mathscr{Z}\left(\mathrm{p}_{1}\right)-\mathscr{Z}\left(\mathrm{p}_{2}\right)\right|^{\beta} \\
& \leq C\left\{\|a\|_{p}+\|b\|_{p}\right\}\left|\mathscr{Z}\left(\mathrm{p}_{1}\right)-\mathscr{Z}\left(\mathrm{p}_{2}\right)\right|^{\beta} \\
& \leq C_{0}\left|\mathscr{Z}\left(\mathrm{p}_{1}\right)-\mathscr{Z}\left(\mathrm{p}_{2}\right)\right|^{\beta}
\end{aligned}
$$

$\operatorname{com} C_{0}:=C\left\{\|a\|_{p}+\|b\|_{p}\right\}$, e $\beta=\frac{2-q-\tau_{\mathrm{M}}}{q}$, onde $\tau_{\mathrm{M}}=\frac{\sigma_{\mathrm{M}}}{\sigma_{\mathrm{M}}+1}$ e $q=\frac{p}{p-1}$.

Das estimativas (6.47) e (6.48) temos que $G\left(\mathscr{B}_{X}\right) \subset \Lambda_{M_{0}, C_{0}}=\Lambda_{M_{0}, C_{0}}(\mathscr{Z}, \Omega, \beta)$, onde $\Lambda_{M_{0}, C_{0}} \subset X$, definido em (5.25) (página 86), é compacto. Segue daí que $G$ é um operador compacto.

Proposição 24. $\operatorname{Ker}(I-G)=\{0\}$.

Demonstração. Precisamos provar que toda solução $w \in C(\bar{\Omega})$ da equação integral

$$
w(x, y)=T_{\mathscr{Z}}(a w+b \bar{w})(x, y)-H(x, y),
$$

com

$$
H(x, y):=\frac{\mathscr{Z}^{2 \kappa+1}(x, y)}{2 \pi i} \int_{\Omega} \frac{(a w+b \bar{w})(\xi, \eta)}{1-\mathscr{Z}(x, y) \overline{\mathscr{Z}(\xi, \eta)}} d \xi d \eta
$$

é a solução trivial $w=0$.

Temos que $H \in C(\bar{\Omega})$ e $L H=0$. Segue da fórmula de representação (Proposição 17, página 77) que para todo $(s, t) \in \Omega$, temos

$$
H(s, t)=\frac{1}{2 \pi i} \int_{\partial \Omega} \frac{H(x, y)}{\mathscr{Z}(x, y)-\mathscr{Z}(s, t)} d \mathscr{Z}(x, y) .
$$

Assim, se $w$ satisfaz a equação (6.49), então, para $(s, t) \in \Omega$,

$$
\frac{1}{2 \pi i} \int_{\partial \Omega} \frac{w(x, y) d \mathscr{Z}(x, y)}{\mathscr{Z}(x, y)-\mathscr{Z}(s, t)}=\frac{1}{2 \pi i} \int_{\partial \Omega} \frac{T_{\mathscr{Z}}(a w+b \bar{w}) d \mathscr{Z}(x, y)}{\mathscr{Z}(x, y)-\mathscr{Z}(s, t)}-H(s, t) .
$$

Pela Proposição 22 (página 115), temos

$$
\int_{\partial \Omega} \frac{T_{\mathscr{Z}}(a w+b \bar{w}) d \mathscr{Z}(x, y)}{\mathscr{Z}(x, y)-\mathscr{Z}(s, t)}=0, \text { para todo }(s, t) \in \Omega .
$$

Assim, a igualdade (6.50) torna-se

$$
\frac{1}{2 \pi i} \int_{\partial \Omega} \frac{w(x, y) d \mathscr{Z}(x, y)}{\mathscr{Z}(x, y)-\mathscr{Z}(s, t)}=-H(s, t), \text { para todo }(s, t) \in \Omega .
$$


Seja $\tilde{H}=H / \mathscr{Z}^{2 \kappa+1}$. Temos que $\tilde{H}: \Omega \rightarrow \mathbb{C}$ é continua e $L \tilde{H}=0$ em $\Omega$. Pelo Teorema 2, página 53, existe $h: \mathbb{D} \rightarrow \mathbb{C}$ holomorfa tal que $\tilde{H}=h \circ \mathscr{Z}$ em $\Omega$. Podemos escrever $h$ em série de potências em $\mathbb{D}$, com expansão em $0 \in \mathbb{D}$. Assim, $H$ pode ser escrita na forma

$$
H(s, t)=\mathscr{Z}^{2 \kappa+1}(s, t) \sum_{j=0}^{\infty} a_{j} \mathscr{Z}^{j}(s, t)
$$

$\operatorname{com} a_{j} \in \mathbb{C}$.

Por outro lado, para $(x, y) \in \partial \Omega \mathrm{e}(s, t) \in \Omega$, temos

$$
\frac{w(x, y)}{\mathscr{Z}(x, y)-\mathscr{Z}(s, t)}=w(x, t) \cdot \frac{1}{\mathscr{Z}(x, y)} \cdot \frac{1}{1-\frac{\mathscr{Z}(s, t)}{\mathscr{Z}(x, y)}}=\frac{w(x, y)}{\mathscr{Z}(x, y)} \sum_{k=0}^{\infty}\left(\frac{\mathscr{Z}(s, t)}{\mathscr{Z}(x, y)}\right)^{k}
$$

ou seja,

$$
\frac{w(x, y)}{\mathscr{Z}(x, y)-\mathscr{Z}(s, t)}=\sum_{k=0}^{\infty} \frac{w(x, y) \mathscr{Z}^{k}(s, t)}{\mathscr{Z}^{k+1}(x, y)} .
$$

Segue então de (6.53), (6.52), e (6.51) que

$$
\int_{\partial \Omega} \frac{w(x, y)}{\mathscr{Z}^{k}(x, y)} \frac{d \mathscr{Z}(x, y)}{\mathscr{Z}(x, y)}=0, \quad k=0,1, \ldots, 2 \kappa .
$$

Para $g \in L^{p}(\Omega), p>2+\sigma_{\mathrm{M}}$, seja

$$
P g(x, y):=\frac{1}{2 \pi i} \int_{\Omega}\left\{\frac{g(\xi, \eta)}{\mathscr{Z}(\xi, \eta)-\mathscr{Z}(x, y)}+\frac{\mathscr{Z}(x, y) \overline{g(\xi, \eta)}}{1-\mathscr{Z}(x, y) \overline{\mathscr{Z}(\xi, \eta)}}\right\} d \xi d \eta .
$$

O operador $P$ tem propriedades semelhantes às de a $T_{\mathscr{Z}}^{K}$ (veja em (6.9), página 103, a definição de $\left.T_{\mathscr{Z}}^{\kappa}\right)$. Em particular,

$$
L(P g)=g \text { em } \Omega \text { e } \mathfrak{I}(P g)=0 \text { em } \partial \Omega ;
$$

além disso, $P g \in C_{\mathscr{Z}}^{\beta}(\bar{\Omega}) \subset C^{\beta}(\bar{\Omega})$.

Como a função $w$ satisfaz (6.49), $w$ também é solução do problema de contorno

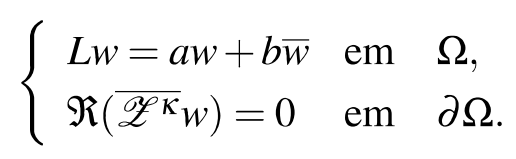

Pelo Princípio da Similaridade, Teorema 10, página 96, $w$ é similar às soluções da equação $L u=0$ em $\Omega$ e portanto tem zeros isolados em $\Omega$. Seja $\psi(x, y)=\bar{w}(x, y) / w(x, y)$ se $w(x, y) \neq 0$ e $\psi(x, y)=0$ caso contrário. Então $\psi \in L^{\infty}(\Omega)$ e $a+b \psi \in L^{p}(\Omega), p>2+\sigma_{\mathrm{M}}$. Para $(x, y) \in \bar{\Omega}$, seja

$$
s(x, y)=P(a+b \psi)(x, y)
$$

Então, a função

$$
\Phi(x, y):=w(x, y) e^{-s(x, y)}
$$

satisfaz $L \Phi=0$ em $\Omega$ (aqui foi usado o Lema 14, página 95). Além disso,

$$
\mathfrak{R}\left(\overline{\mathscr{Z}} \kappa_{\Phi}\right)=e^{-\mathfrak{R}(s)} \mathfrak{R}\left(\overline{\mathscr{Z}} \kappa_{w}\right)=0 \text { em } \partial \Omega .
$$


Pela Proposição 21 (página 108), podemos escrever $\Phi$ da forma

$$
\Phi(x, y)=\sum_{j=0}^{2 \kappa} b_{j} \mathscr{Z}^{j}(x, y),
$$

$\operatorname{com} b_{j} \in \mathbb{C}, b_{2 \kappa-j}=-\bar{b}_{j}$ para $j=0,1, \ldots, 2 \kappa$. Segue então que a função $w=\Phi e^{s}$ tem a forma

$$
w(x, y)=\left(\sum_{j=0}^{2 \kappa} b_{j} \mathscr{Z}^{j}(x, y)\right) e^{s(x, y)}
$$

Com a expressão de $w$ dada por (6.57), podemos reescrever (6.54) como

$$
\sum_{k=0}^{2 \kappa} b_{k} \int_{\partial \Omega} \mathscr{Z}^{k-j}(x, y) e^{s(x, y)} \frac{d \mathscr{Z}(x, y)}{\mathscr{Z}(x, y)}=0, j=0,1, \ldots, 2 \kappa .
$$

Então, mudando de variável, (usando o Lema 9, página 77) temos

$$
\sum_{k=0}^{2 \kappa} b_{k} \int_{\partial \mathbb{D}} \zeta^{k-j} e^{\widetilde{s}(\zeta)} \frac{d \zeta}{\zeta}=0, j=0,1, \ldots, 2 \kappa
$$

$\operatorname{com} \tilde{s}=s \circ \mathscr{Z}^{-1}, \operatorname{com} \tilde{s}(\zeta)=\overline{\tilde{s}(\zeta)}, \zeta \in \partial \mathbb{D}$.

Seja $\mathscr{P}(\mathbb{D})=\left\{u \in C(\overline{\mathbb{D}}) ; u(\zeta)=a_{0}+a_{1} \zeta+\ldots+a_{2 \kappa} \zeta^{2 \kappa}, a_{1}, \ldots, a_{2 \kappa} \in \mathbb{C}, \zeta \in \mathbb{D}\right\}$. Considere o produto interno $\langle\cdot, \cdot\rangle: \mathscr{P}(\mathbb{D}) \times \mathscr{P}(\mathbb{D}) \rightarrow \mathbb{C}$ dado por

$$
\langle u, v\rangle:=\frac{1}{2 \pi i} \int_{\partial \mathbb{D}} u(\zeta) \overline{v(\zeta)} e^{\widetilde{s}(\zeta)} \frac{d \zeta}{\zeta} .
$$

Assim, podemos reescrever (6.59) como

$$
\operatorname{Gr}\left(v_{1}, \ldots, v_{2 \kappa}\right) v=0
$$

onde

com

$$
\operatorname{Gr}\left(v_{1}, \ldots, v_{2 \kappa}\right)=\left[\begin{array}{cccc}
\left\langle v_{0}, v_{0}\right\rangle & \left\langle v_{0}, v_{1}\right\rangle & \cdots & \left\langle v_{0}, v_{2 \kappa}\right\rangle \\
\left\langle v_{1}, v_{0}\right\rangle & \left\langle v_{1}, v_{1}\right\rangle & \cdots & \left\langle v_{1}, v_{2 \kappa}\right\rangle \\
\vdots & \vdots & \ddots & \vdots \\
\left\langle v_{2 \kappa}, v_{0}\right\rangle & \left\langle v_{2 \kappa}, v_{1}\right\rangle & \cdots & \left\langle v_{2 \kappa}, v_{2 \kappa}\right\rangle
\end{array}\right] \mathrm{e} \mathrm{v}=\left[\begin{array}{c}
b_{0} \\
b_{1} \\
\vdots \\
b_{2 \kappa}
\end{array}\right]
$$

$$
v_{j}(\zeta)=\zeta^{j}, j=0,1,2, \ldots, 2 \kappa .
$$

A matriz $\operatorname{Gr}\left(v_{1}, \ldots, v_{2 \kappa}\right)$ é chamada matriz de Gramm do conjunto de vetores em (6.60). Pela independência linear dos vetores em (6.60), temos que o determinante de $\operatorname{Gr}\left(v_{1}, \ldots, v_{2 \kappa}\right)$ é não nulo (ver Teorema 24, página 132). Logo, v =0, ou seja,

$$
b_{k}=0, \text { para } k=0,1, \ldots, 2 \kappa .
$$

Assim, segue de (6.57) que $w=0$. 
Assim, temos o seguinte teorema

Teorema 16. Sejam $a, b, f \in L^{p}(\Omega), p>2+\sigma_{\mathrm{M}}$, e $\varphi \in C^{\alpha}(\partial \Omega, \mathbb{R}), 0<\alpha<1$. O problema de contorno (6.44) tem uma única solução $u \in C_{\mathscr{Z}}^{\alpha_{0}}(\bar{\Omega}) \subset C^{\alpha_{0}}(\bar{\Omega}), \operatorname{com} \alpha_{0}=\min \left\{\alpha,\left(2-q-\tau_{\mathrm{M}}\right) / q\right\}$, onde $q=p /(p-1)$.

Demonstração. Pela Proposição 21 (página 108) existe $\Phi \in C_{\mathscr{Z}}^{\alpha}(\bar{\Omega})$ satisfazendo $L \Phi=0$ em $\Omega$ e $\Re(\overline{\mathscr{Z} \kappa} \Phi)=\varphi$ em $\partial \Omega$.

Como $G$ é compacto (Proposição 23), $\operatorname{Ker}(G-I)=\{0\}$ (Proposição 24) e $\psi=T_{\mathscr{Z}}^{K} f+$ $\Phi \in X$, pelo Teorema de Fredholm (Teorema 27, página 133) temos que existe $u \in X$ tal que

$$
(G-I) u=\psi
$$

Ou seja, existe $u \in C(\bar{\Omega})$ tal que

$$
u=T_{\mathscr{Z}}^{\kappa}(a u+b \bar{u})+T_{\mathscr{Z}}^{\kappa}(f)+\Phi \operatorname{em} \Omega
$$

Segue da expressão acima que $u \in C_{\mathscr{Z}}^{\alpha_{0}}(\bar{\Omega}) \subset C^{\alpha_{0}}(\bar{\Omega})$, com $\alpha_{0}=\min \left\{\alpha,\left(2-q-\tau_{\mathrm{M}}\right) / q\right\}$, onde $q=p /(p-1)$.

Por (6.61) e pela Proposição 19 (página 106)

$$
\mathfrak{R}\left(\overline{\mathscr{Z}}^{\kappa} u\right)=\mathfrak{R}(\overline{\mathscr{Z} \kappa} \Phi)=\varphi \operatorname{em} \partial \Omega
$$

Pela Proposição 12 (página 104)

$$
L u=a u+b \bar{u}+f \text { em } \Omega .
$$

A unicidade da solução segue da Proposição 24.

Vamos agora considerar o seguinte problema de Riemann-Hilbert mais geral do que o tratado anteriormente

$$
\left\{\begin{array}{lll}
L u=a u+b \bar{u}+f & \text { em } & \Omega, \\
\Re(\bar{\Lambda} u)=\varphi & \text { em } & \partial \Omega,
\end{array}\right.
$$

$\operatorname{com} a, b, f \in L^{p}(\Omega), p>2+\sigma_{\mathrm{M}}, \Lambda \in C^{\alpha}(\partial \Omega),|\Lambda|=1, \varphi \in C^{\alpha}(\partial \Omega ; \mathbb{R}), 0<\alpha<1$.

O próximo teorema trata de uma versão do problema de Riemann-Hilbert que é mais geral do que a versão que foi vista no último teorema, a saber, o problema (6.62). A existência de solução do problema está relacionada com o índice de $\Lambda$ associado a $L$ com respeito a $\partial \Omega$. Se $L$ é expresso por $L=A \partial / \partial x+B \partial / \partial y$ no aberto $\tilde{\Omega}$ (que contém $\bar{\Omega}$ ), então o índice de $\Lambda$ associado a $L$ com respeito a $\partial \Omega$ é dado por

$$
\operatorname{Ind}_{L, \partial \Omega}(\Lambda)=-\operatorname{sgn}\left(\mathfrak{I}(A \bar{B})\left(\mathrm{p}_{0}\right)\right) \operatorname{ind}_{\partial \Omega}(\Lambda),
$$


onde $\mathrm{p}_{0}$ é um ponto qualquer de $\tilde{\Omega} \backslash \Sigma$ e sgn denota a função sinal, isto é, sgn : $\mathbb{R} \backslash\{0\} \rightarrow\{-1,1\}$, $\operatorname{sgn}(x)=1$ se $x>0$ e $\operatorname{sgn}(x)=-1$ se $x<0$. Quando $-\operatorname{sgn}\left(\mathfrak{I}(A \bar{B})\left(\mathrm{p}_{0}\right)\right)>0$, a aplicação $\mathscr{Z}: \bar{\Omega} \rightarrow \overline{\mathbb{D}}$ preserva orientação e quando $-\operatorname{sgn}\left(\mathfrak{I}(A \bar{B})\left(\mathrm{p}_{0}\right)\right)<0$ a aplicação $\mathscr{Z}$ inverte a orientação. O índice de $\Lambda$ com respeito a $\partial \Omega$, ind $\partial \Omega(\Lambda)$, é definido na definição 8 (página 39 ). (Veja mais na Seção 2.4, página 38).

De modo análogo ao que foi feito na demonstração do Teorema 15 (página 114), vamos fazer uma mudança de incógnita, usando o índice $\operatorname{Ind}_{L, \partial \Omega}(\Lambda)$, para obter uma solução do problema (6.62) a partir de uma solução do problema (6.44).

Teorema 17. Se $\operatorname{Ind}_{L, \partial \Omega}(\Lambda) \geq 0$, então o problema de Riemann-Hilbert (6.62) tem uma única solução $u \in C_{\mathscr{Z}}^{\alpha_{0}}(\bar{\Omega}) \subset C^{\alpha_{0}}(\bar{\Omega})$ (com $\alpha_{0}$ como no Teorema 16).

Demonstração. Seja $\kappa=\operatorname{Ind}_{L, \partial \Omega}(\Lambda) \geq 0$. Vamos fazer uma mudança de incógnita usando o operador de Schwarz $S_{\mathscr{Z}}$ (definido in (6.24), página 107) para trocar a função da condição de contorno $\Lambda$ por $\mathscr{Z}^{\kappa}$ e assim poder aplicar o teorema anterior (Teorema 16).

Seja $\kappa:=\operatorname{Ind}_{L, \partial \Omega}(\Lambda)$ e seja $g: \partial \Omega \rightarrow \mathbb{S}^{1} \subset \mathbb{C}$ dada por

$$
g(x, y)=\mathscr{Z}^{-\kappa}(x, y) \Lambda(x, y) .
$$

Temos que $\operatorname{Ind}_{L, \partial \Omega}(g)=0$. Consequentemente, existe uma função $\vartheta: \partial \Omega \rightarrow \mathbb{R}$ Hölder contínua (com mesmo expoente de Hölder que $g$ ) tal que

$$
g(x, y)=e^{i \vartheta(x, y)}, \text { para todo }(x, y) \in \partial \Omega
$$

$\operatorname{Para}(x, y) \in \bar{\Omega}$ defina

$$
\gamma(x, y)=\left(S_{\mathscr{Z}} \vartheta\right)(x, y) .
$$

Então $L \gamma=0$ em $\Omega$ e $\Re(\gamma)=\vartheta$ em $\partial \Omega$ (Proposição 20, página 107). Assim,

$$
e^{-i \gamma}=e^{\mathfrak{I}(\gamma)+i \kappa \arg (\mathscr{Z})-i \arg (\Lambda)}=e^{\mathfrak{I}(\gamma) \mathscr{Z}^{\kappa} \bar{\Lambda}}
$$

$\operatorname{Sejam} \tilde{b}=b \frac{\overline{e^{i \gamma}}}{e^{i \gamma}}$ e $\tilde{f}=f e^{-i \gamma}$. Então $\tilde{b}, \tilde{f} \in L^{p}(\Omega), p>2+\sigma_{\mathrm{M}}$ e pelo Teorema 16 o problema

$$
\left\{\begin{array}{lll}
L U=a U+\tilde{b} \bar{U}+\tilde{f} & \text { em } & \Omega, \\
\Re(\overline{\mathscr{Z} \kappa} U)=e^{\mathfrak{I}(\gamma)} \varphi & \text { em } & \partial \Omega,
\end{array}\right.
$$

tem solução $U \in C^{\alpha_{0}}(\bar{\Omega}), \operatorname{com} \alpha_{0}=\min \left\{\alpha,\left(2-q-\tau_{\mathrm{M}}\right) / q\right\}$. Defina $u=e^{i \gamma} U$ em $\bar{\Omega}$. Temos

$$
L u=e^{i \gamma} L U=e^{i \gamma}\{a U+\tilde{b} \bar{U}+\tilde{f}\}=a u+b \bar{u}+f \operatorname{em} \Omega,
$$

Além disso, por (6.63) e (6.64),

$$
\mathfrak{R}(\bar{\Lambda} u)=e^{-\mathfrak{I}(\gamma)} \mathfrak{R}(\overline{\mathscr{Z} \kappa} u)=\varphi \text { em } \partial \Omega .
$$

Isto completa a prova do teorema. 
Observação 27. A existência de solução para o Problema de Riemann-Hilbert (6.62) quando $\kappa=\operatorname{Ind}_{L, \partial \Omega} \Lambda<0$ depende do estudo de condições de compatibilidade que envolvem o operador de Schwarz $S_{\mathscr{Z}}$, as condições de contorno do problema e o operador $T_{\mathscr{Z}}^{\kappa}$. Veja como exemplo o caso clássico descrito na Observação 36, página 141.

\subsection{O problema de Riemann-Hilbert para a equação $L u=$ $a u+f$ quando $L$ é hipoelítico}

Seja $\tilde{\Omega}$ um aberto conexo de $\mathbb{R}^{2}$ e $L$ um campo vetorial complexo de classe $C^{\infty}$ não singular definido em $\tilde{\Omega}$, isto é,

$$
L=A(x, y) \frac{\partial}{\partial x}+B(x, y) \frac{\partial}{\partial y}
$$

$\operatorname{com} A, B \in C^{\infty}(\tilde{\Omega})$ e $|A|+|B|>0$ em $\Omega$. Seja $\Omega$ um aberto simplesmente conexo com bordo suave de $\mathbb{R}^{2}$ tal que $\bar{\Omega}$ é um compacto de $\mathbb{R}^{2}$ e $\bar{\Omega} \subset \tilde{\Omega}$.

Vamos supor nesta seção que $L$ é hipoelítico ${ }^{1}$ em $\Omega$.

Nesta seção vamos considerar o seguinte problema de Riemann-Hilbert:

$$
\left\{\begin{array}{lll}
L u=a u+f & \text { em } & \Omega, \\
\Re(\bar{\Lambda} u)=\varphi & \text { em } & \partial \Omega,
\end{array}\right.
$$

onde $a, f \in C^{\infty}(\tilde{\Omega}), \Lambda, \varphi \in C^{\alpha}(\partial \Omega), 0<\alpha<1, \operatorname{com}|\Lambda|=1$.

Na Seção 3.3, página 56, definimos hipoeliticidade de um campo vetorial complexo e relacionamos este conceito com a classe de campos estudada neste texto.

Esta seção ilustra, através do estudo do problema acima, o caso em que a equação diferencial, do tipo de problema de contorno estudado nesta tese, é $\mathbb{C}$-linear em relação a "variável" $u$. Neste caso a abordagem do problema é mais simples e não é necessário utilizar a teoria desenvolvida para o operador integral $T_{Z}$. A existência de solução do problema será consequência da teoria clássica, da resolubilidade local de $L$, uma vez que campos hipoelíticos (não singulares) satisfazem a condição (P), e da existência de uma integral primeira global de $L$ que é um homeomorfismo sobre sua imagem.

Assinalamos que não haverá hipóteses sobre o conjunto característico como nos problemas anteriores.

\subsubsection{Resolubilidade global para campos hipoelíticos no plano}

Sejam $\tilde{\Omega}$ e $L$ como anteriormente. Nesta parte estudaremos um pouco sobre a resolubilidade de $L$.

\footnotetext{
1 veja a definição 11 , página 57.
} 
Definição 12. Um campo vetorial complexo $L$ é dito localmente resolúvel em $\Omega$, se para todo ponto $\mathrm{p} \in \tilde{\Omega}$ existe $\mathscr{O}_{\mathrm{p}}$ um aberto de $\tilde{\Omega}$, com $\mathrm{p} \in \mathscr{O}_{\mathrm{p}}$, e $u_{\mathrm{p}} \in \mathscr{D}^{\prime}\left(\mathscr{O}_{\mathrm{p}}\right)$ tal que $L u_{\mathrm{p}}=f$ em $\mathscr{O}_{\mathrm{p}}$.

Um campo vetorial não singular hipoelítico é localmente resolúvel. Isso segue do fato de que todo campo vetorial não singular hipoelítico satisfaz a condição $(P)$ de Nirenberg e Treves. (Veja (TREVES, 1971)). A condição (P) é necessária e suficiente para resolubilidade local (veja em (BERHANU; CORDARO; HOUNIE, 2008)).

Definição 13. Um campo vetorial $L$ é dito globalmente resolúvel em $\tilde{\Omega}$ se para toda $f \in C^{\infty}(\tilde{\Omega})$ existe uma distribuição $u \in \mathscr{D}^{\prime}(\tilde{\Omega})$ tal que $L u=f \mathrm{em} \tilde{\Omega}$.

Note que, se $L$ é um campo vetorial hipoelítico em $\tilde{\Omega}$ e $f \in C^{\infty}(\tilde{\Omega})$, então toda solução $u$ da equação $L u=f$ em $\Omega$ é uma função de classe $C^{\infty}$ em $\tilde{\Omega}$.

Teorema 18. Para toda $f \in C^{\infty}(\tilde{\Omega})$ existe uma função $u \in C^{\infty}(\tilde{\Omega})$ tal que $\partial u / \partial \bar{z}=f$ em $\tilde{\Omega}$.

Demonstração. Veja (HöRMANDER, 1973), página 12, Teorema 1.4.4.

Observação 28. A demonstração do resultado acima, Teorema 18, que se encontra em (HöRMANDER, 1973), não é uma consequência imediata do Teorema 32, página 138.

Teorema 19. Se $L$ é um campo hipoelítico não singular em $\Omega$, então $L$ é globalmente resolúvel em $\tilde{\Omega}$, i.e., para toda $f \in C^{\infty}(\tilde{\Omega})$ existe uma função $u \in C^{\infty}(\tilde{\Omega})$ tal que $L u=f$ em $\tilde{\Omega}$.

Demonstração. Como $L$ é um campo vetorial hipoelítico não singular em $\tilde{\Omega}$, existe $Z: \tilde{\Omega} \rightarrow \mathbb{C}$, de classe $C^{\infty}$, tal que $L Z=0$ em $\tilde{\Omega}, d Z \neq 0$ em $\tilde{\Omega}$ e $Z: \tilde{\Omega} \rightarrow Z(\tilde{\Omega})$ é um homeomorfismo. (Veja a Proposição 13, na página 57).

Seja $f \in C^{\infty}(\tilde{\Omega})$. Como $L$ é hipoelítico (e localmente resolúvel) existe uma cobertura aberta $\left\{\mathscr{O}_{v}\right\}_{v=1}^{\infty}$ de $\tilde{\Omega}$ tal que para cada $v$ natural existe $u_{v} \in C^{\infty}\left(\mathscr{O}_{v}\right)$ que satisfaz $L u_{v}=f$ em $\mathscr{O}_{v}$.

Se $\mathscr{O}_{j} \cap \mathscr{O}_{k} \neq 0$ então $L\left(u_{j}-u_{k}\right)=f-f=0$ em $\mathscr{O}_{j} \cap \mathscr{O}_{k}$. Assim, pelo Teorema 3 (página 57), existe $h_{j k}: Z\left(\mathscr{O}_{j}\right) \cap Z\left(\mathscr{O}_{k}\right) \rightarrow \mathbb{C}$ holomorfa tal que

$$
\left(u_{j}-u_{k}\right)(\mathrm{p})=h_{j k}(Z(\mathrm{p})) \operatorname{em} Z\left(\mathscr{O}_{j}\right) \cap Z\left(\mathscr{O}_{k}\right)
$$

A família $\left\{h_{j k}\right\}$ satisfaz:

(a) $h_{j j}=0 \mathrm{em} Z\left(\mathscr{O}_{j}\right), j \in \mathbb{N}$;

(b) $h_{j k}=-h_{k j} \operatorname{em~} Z\left(\mathscr{O}_{j}\right) \cap Z\left(\mathscr{O}_{k}\right), j, k \in \mathbb{N}$;

(c) $h_{j k}+h_{k l}+h_{l j}=0$ em $Z\left(\mathscr{O}_{j}\right) \cap Z\left(\mathscr{O}_{k}\right) \cap Z\left(\mathscr{O}_{l}\right), j, k, l \in \mathbb{N}$. 
Seja $\left\{\varphi_{v}\right\}$ uma partição da unidade subordinada à cobertura aberta $\left\{Z\left(\mathscr{O}_{v}\right)\right\}_{v=1}^{\infty}$ de $Z(\tilde{\Omega})$. Para cada $k \in \mathbb{N}$, seja

$$
w_{k}:=\sum_{\nu=1}^{\infty} \varphi_{v} h_{v k} \operatorname{em~} Z(\tilde{\Omega}) .
$$

A função $w_{k}$ está bem definida e $w_{k} \in C^{\infty}(Z(\tilde{\Omega}))$. Por (b) e (c) temos

$$
w_{k}-w_{j}=\sum_{v=1}^{\infty} \varphi_{v}\left(h_{v k}-h_{v j}\right)=\sum_{v=1}^{\infty} \varphi_{v} h_{j k}=h_{j k} \sum_{v=1}^{\infty} \varphi_{v}=h_{j k}
$$

em $Z\left(\mathscr{O}_{k}\right) \cap Z\left(\mathscr{O}_{j}\right), j, k \in \mathbb{N}$. Por (6.67) temos

$$
\frac{\partial w_{k}}{\partial \bar{z}}=\frac{\partial w_{j}}{\partial \bar{z}} \text { em } Z\left(\mathscr{O}_{k}\right) \cap Z\left(\mathscr{O}_{j}\right), j, k \in \mathbb{N}
$$

Assim, existe uma função $\Psi \in C^{\infty}(Z(\tilde{\Omega}))$ tal que

$$
\left.\Psi\right|_{Z\left(\mathscr{O}_{k}\right)}=\frac{\partial w_{k}}{\partial \bar{z}} \text { em } Z\left(\mathscr{O}_{k}\right) \forall k \in \mathbb{N}
$$

Como o operador de Cauchy-Riemann é globalmente resolúvel (veja o Teorema 18) existe $U \in C^{\infty}(\tilde{\Omega})$ tal que

$$
\frac{\partial U}{\partial \bar{z}}=-\Psi \operatorname{em~} Z(\tilde{\Omega})
$$

Seja $h_{k}:=w_{k}+U$ em $Z\left(\mathscr{O}_{k}\right)$. Assim

$$
\frac{\partial h_{k}}{\partial \bar{z}}=\frac{\partial w_{k}}{\partial \bar{z}}+\frac{\partial U}{\partial \bar{z}}=\Psi-\Psi=0 \text { em } Z\left(\mathscr{O}_{k}\right), \quad \forall k \in \mathbb{N} .
$$

Portanto $h_{k}$ é holomorfa em $Z\left(\mathscr{O}_{k}\right), k \in \mathbb{N}$. Além disso, temos por (6.67)

$$
h_{k}-h_{j}=w_{k}+U-w_{j}-U=w_{k}-w_{j}=h_{k j} \text { em } Z\left(\mathscr{O}_{k}\right) \cap Z\left(\mathscr{O}_{j}\right), \quad \forall j, k \in \mathbb{N} .
$$

Assim podemos definir a função $u: \tilde{\Omega} \rightarrow \mathbb{C}$ dada por

$$
u(\mathrm{p})=u_{j}(\mathrm{p})-h_{j}(Z(\mathrm{p})), \text { se } \mathrm{p} \in \mathscr{O}_{j}
$$

Por (6.68) $u$ está bem definida. De fato, seja $\mathrm{p} \in \mathscr{O}_{k} \cap \mathscr{O}_{j}$. Então, por (6.66) e (6.68), temos

$$
\begin{aligned}
& \left(u_{k}(\mathrm{p})-h_{k}(Z(\mathrm{p}))\right)-\left(u_{j}(\mathrm{p})-h_{j}(Z(\mathrm{p}))\right)=u_{k}(\mathrm{p})-u_{j}(\mathrm{p})-\left(h_{k}(Z(\mathrm{p}))-h_{j}(Z(\mathrm{p}))\right)= \\
& \left(u_{k}(\mathrm{p})-u_{j}(\mathrm{p})\right)-\left(h_{k}(Z(\mathrm{p}))-h_{j}(Z(\mathrm{p}))\right)=h_{k j}(Z(\mathrm{p}))-h_{k j}(Z(\mathrm{p}))=0 .
\end{aligned}
$$

Note que $u \in C^{\infty}(\tilde{\Omega})$. Agora observe que, se $\mathrm{p} \in \tilde{\Omega}$, então existe $j \in \mathbb{N}$ tal que $\mathrm{p} \in \mathscr{O}_{j}$ e

$$
L u=L u_{j}+L\left(h_{j} \circ Z\right)=f \text { em } \mathscr{O}_{j} .
$$

Como $\left\{\mathscr{O}_{v}\right\}_{v=1}^{\infty}$ é uma cobertura de $\tilde{\Omega}$, temos que $L u=f$ em $\tilde{\Omega}$. 


\subsubsection{O problema de Riemann-Hilbert para a equação $L u=a u+f$}

Seja $L$ um campo hipoelítico não singular definido em um aberto $\Omega$ de $\mathbb{R}^{2}$ e $\Omega$ um aberto simplesmente conexo de $\tilde{\Omega}$ tal que $\bar{\Omega}$ é um compacto de $\mathbb{R}^{2}$, com $\bar{\Omega} \subset \tilde{\Omega}$ e $\partial \Omega$ é uma variedade mergulhada de dimensão 1 de $\mathbb{R}^{2}$, de classe $C^{1+\varepsilon}, 0<\varepsilon<1$, transversal a $L$ no seguinte sentido

$$
i^{*} \omega \neq 0
$$

onde $\omega=B d x-A d y$ é ortogonal a $L=A \partial / \partial x+B \partial / \partial y$ e $i: \Omega \rightarrow \mathbb{R}^{2}$ é a aplicação de inclusão. (Veja a Seção 2.5, página 44).

Seja $Z: \tilde{\Omega} \rightarrow \mathbb{C}$ uma integral primeira global de $L$, de classe $C^{\infty}$, que é um homeomorfismo sobre sua imagem.

Nesta seção vamos provar a existência de solução para o seguinte problema de contorno

$$
\left\{\begin{array}{lll}
L u=a u+f & \text { em } & \Omega, \\
\Re(\bar{\Lambda} u)=\varphi & \text { em } & \partial \Omega,
\end{array}\right.
$$

onde $\Lambda \in C^{\alpha}(\partial \Omega), \operatorname{com}|\Lambda|=1, \varphi \in C^{\alpha}(\partial \Omega, \mathbb{R}), 0<\alpha<1$ e $f, a \in C^{\infty}(\tilde{\Omega})$.

Vamos relembrar o conceito de índice usado neste texto. Se $L$ é expresso por $L=$ $A \partial / \partial x+B \partial / \partial y$ no aberto $\Omega$, então o índice de $\Lambda$ associado a $L$ com respeito a $\partial \Omega$ é dado por

$$
\operatorname{Ind}_{L, \partial \Omega}(\Lambda)=-\operatorname{sgn}\left(\mathfrak{I}(A \bar{B})\left(\mathrm{p}_{0}\right)\right) \operatorname{ind}_{\partial \Omega}(\Lambda),
$$

onde $\mathrm{p}_{0}$ é um ponto qualquer de $\tilde{\Omega} \backslash \Sigma$, onde $\Sigma$ é o conjunto característico de $L$, e sgn denota a função sinal, isto é, sgn : $\mathbb{R} \backslash\{0\} \rightarrow\{-1,1\}, \operatorname{sgn}(x)=1$ se $x>0$ e $\operatorname{sgn}(x)=-1$ se $x<0$. Quando $-\operatorname{sgn}\left(\mathfrak{I}(A \bar{B})\left(\mathrm{p}_{0}\right)\right)>0$, a integral primeira $Z: \tilde{\Omega} \rightarrow \mathbb{C}$ preserva orientação e quando $-\operatorname{sgn}\left(\mathfrak{I}(A \bar{B})\left(\mathrm{p}_{0}\right)\right)<0$ a integral primeira $Z$ inverte a orientação. O índice de $\Lambda$ com respeito a $\partial \Omega$, ind $\partial \Omega(\Lambda)$, é definido na definição 8 (página 39). (Veja mais na Seção 2.4, página 38).

Teorema 20. Se o índice $\operatorname{Ind}_{L, \partial \Omega}(\Lambda) \geq 0$, então o problema (6.69) possui uma solução $u \in$ $C^{\infty}(\Omega) \cap C^{\alpha}(\bar{\Omega})$.

Demonstração. Pelo Teorema 19, que fala sobre a resolubilidade global de campos hipoelíticos, existem $v \in C^{\infty}(\tilde{\Omega})$ tal que $L v=a \operatorname{e~} V \in C^{\infty}(\tilde{\Omega})$ tal que $L V=f e^{-v}$. Então $U:=V e^{v}$ é de classe $C^{\infty}$ em $\tilde{\Omega}$ e satisfaz

$$
L U=(L V) e^{v}+V e^{v} L v=f e^{-v} e^{v}+V e^{v} a=f+U a=a U+f \text { em } \tilde{\Omega} .
$$

Agora considere o problema

$$
\left\{\begin{array}{lll}
L H=0 & \text { em } & \Omega, \\
\Re(\bar{\chi} H)=\theta & \text { em } & \partial \Omega,
\end{array}\right.
$$

onde $\chi=\Lambda e^{v}$ e $\theta=\varphi-\Re(\bar{\Lambda} U)$. Temos que $\chi, \theta \in C^{\alpha}(\partial \Omega)$. Temos também que $\operatorname{Ind}_{L, \partial \Omega}(\chi) \geq 0$, uma vez que $\operatorname{Ind}_{L, \partial \Omega}(\Lambda) \geq 0$ e $\left|e^{v}\right|>0$ (veja (2.15), página 41). 
Sejam $\tilde{\chi}=\chi \circ Z^{-1}$ e $\tilde{\theta}=\theta \circ Z^{-1}$. Temos $\tilde{\chi}, \tilde{\theta} \in C^{\alpha}(Z(\partial \Omega))$ pela transversalidade de $\partial \Omega$ em relação a $L$ (lema 3, página 44). Como $\operatorname{Ind}_{L, \partial \Omega}(\chi) \geq 0$ temos o índice clássico $\operatorname{ind}_{Z(\partial \Omega)}(\tilde{\chi}) \geq 0$. Pela teoria clássica do problema de Riemann-Hilbert (veja (VEKUA, 1962) e (BEGEHR, 1994); veja também o Corolário 11, página 141), temos que existe $h \in C^{\alpha}(Z(\bar{\Omega}))$ tal que

$$
\left\{\begin{array}{l}
\frac{\partial h}{\partial \bar{z}}=0 \quad \text { em } \quad Z(\Omega), \\
\Re(\overline{\tilde{\chi}} h)=\tilde{\theta} \quad \text { em } \quad Z(\partial \Omega) .
\end{array}\right.
$$

Defina $H: \bar{\Omega} \rightarrow \mathbb{C}$ por $H=h \circ Z$. Temos que $L H=0$ em $\Omega$ pois $h$ é holomorfa em $Z(\Omega)$ e $L Z=0$ em $\Omega$. (Veja a Proposição 2, página 31).

Seja $(x, y) \in \partial \Omega$. Temos

$$
\mathfrak{R}(\bar{\chi} H(x, y))=\mathfrak{R}(\overline{\tilde{\chi}(Z(x, y))} h(Z(x, y)))=\tilde{\theta}(Z(x, y))=\theta(x, y)=\{\varphi-\Re(\bar{\Lambda} U)\}(x, y) .
$$

Portanto $H$ é uma solução do problema (6.70).

Seja $u:=H e^{v}+U$ em $\bar{\Omega}$. Temos que $u \in C^{\alpha}(\bar{\Omega}) \cap C^{\infty}(\Omega)$ e

$$
L u=L\left(H e^{v}\right)+L U=a H e^{v}+a U+f=a\left(H e^{v}+U\right)+f=a u+f \operatorname{em} \Omega .
$$

A condição de contorno:

$$
\mathfrak{R}(\bar{\Lambda} u)=\mathfrak{R}\left(\bar{\Lambda} H e^{v}\right)+\mathfrak{R}(\bar{\Lambda} U)=\mathfrak{R}\left(\bar{\Lambda} e^{v} H\right)+\mathfrak{R}(\bar{\Lambda} U)=\varphi-\mathfrak{R}(\bar{\Lambda} U)+\mathfrak{R}(\bar{\Lambda} U)=\varphi \operatorname{em} \partial \Omega
$$

Portanto, $u$ é uma solução do problema (6.69). 



\section{A}

\section{TEOREMAS IMPORTANTES USADOS NO}

\section{Teoremas de Riemann}

Teorema 21. (Teorema da Aplicação de Riemann e Suavidade Sobre o Bordo) Seja $D$ um aberto limitado simplesmente conexo de $\mathbb{C}$ com bordo suave de classe $C^{k+\varepsilon}, k=1,2, \ldots$ e $0<\varepsilon<1$. Então, um biholomorfismo de $D$ sobre $\mathbb{D}$ que se estende a um difeomorfismo de classe $C^{k}$, de $\bar{D}$ sobre $\overline{\mathbb{D}}$.

Veja em (POMMERENKE, 1992), (KRANTZ, 2006).

Teorema 22. Seja $S$ uma superfície de Riemann que é homeomorfa a um aberto de $\mathbb{R}^{2}$. Então existe uma aplicação holomorfa injetiva $f: S \rightarrow \mathbb{C}$.

Veja em (SPRINGER, 1957).

Observação 29. O termo "holomorfa" usado no enunciado do teorema acima é referente a estrutura de superfície de Reimann de $S$. Dizemos que $f: S \rightarrow \mathbb{C}$ é holomorfa se para capa ponto $\mathrm{p} \in S$ existe uma carta $(U, \Phi)$ de $S$, com $\mathrm{p} \in U$, tal que

$$
f \circ \Phi^{-1}: \Phi(U) \rightarrow \mathbb{C}
$$

é uma função holomorfa (no sentido usual da análise complexa).

Veja em (FARKAS; KRA, 1980) teoremas semelhantes ao Teorema 22. Tais teoremas são chamados de teoremas de uniformização. 


\section{Extensão de funções holomorfas}

Um arco de Jordan é a imagem de intervalo fechado em $\mathbb{R}$ por uma aplicação contínua e injetiva chegando em $\mathbb{C}$.

Teorema 23. Seja $D$ um aberto conexo de $\mathbb{C}$ e seja $\Gamma$ um arco de Jordan retificável que divide $D$ em dois abertos conexos $D_{1}$ e $D_{2}$. Suponha que $f_{j}: \Gamma \cup D_{j} \rightarrow \mathbb{C}, j=1$, 2, é contínua e holomorfa em $D_{j}, j=1,2$. Então a função $f: D \rightarrow \mathbb{C}$ definida por $f=f_{j}$ em $\Gamma \cup D_{j}, j=1,2$, é holomorfa em $D$.

Veja em (ZALCMAN, 1974).

\section{Teorema de Gramm}

Seja $E$ um espaço vetorial de dimensão $n$. Seja $\langle\cdot, \cdot\rangle: E \times E \rightarrow \mathbb{C}$ um produto interno em $E$. A matriz de Gramm do conjunto de vetores $v_{1}, v_{2}, \ldots, v_{n} \in E$ é a matriz $\operatorname{Gr}\left(v_{1}, \ldots, v_{n}\right), n \times n$, dada por

$$
\operatorname{Gr}\left(v_{1}, \ldots, v_{n}\right)=\left[\begin{array}{cccc}
\left\langle v_{1}, v_{1}\right\rangle & \left\langle v_{1}, v_{2}\right\rangle & \cdots & \left\langle v_{1}, v_{n}\right\rangle \\
\left\langle v_{2}, v_{1}\right\rangle & \left\langle v_{2}, v_{2}\right\rangle & \cdots & \left\langle v_{2}, v_{n}\right\rangle \\
\vdots & \vdots & \ddots & \vdots \\
\left\langle v_{n}, v_{1}\right\rangle & \left\langle v_{n}, v_{2}\right\rangle & \cdots & \left\langle v_{n}, v_{n}\right\rangle
\end{array}\right]
$$

Teorema 24. Os vetores $v_{1}, v_{2}, \ldots, v_{n}$ são linearmente independentes em $E$ se, e somente se, a sua matriz de $\operatorname{Gramm} \operatorname{Gr}\left(v_{1}, \ldots, v_{n}\right)$ é não singular.

Demonstração. Suponha que $v_{1}, v_{2}, \ldots, v_{n}$ são linearmente dependentes. Então existe um vetor não nulo $a=\left(a_{k}\right)_{k=1, \ldots, n} \in \mathbb{C}^{n}$ tal que

$$
\sum_{k=1}^{n} a_{k} v_{k}=0
$$

Então, para todo $j=1,2, \ldots, n$,

$$
\sum_{k=1}^{n} a_{k}\left\langle v_{j}, v_{k}\right\rangle=0 .
$$

Ou seja, $\operatorname{Gr}\left(v_{1}, \ldots, v_{n}\right) a=0$. Portanto, $\operatorname{Gr}\left(v_{1}, \ldots, v_{n}\right)$ é singular.

Reciprocamente, suponha que $v_{1}, v_{2}, \ldots, v_{n}$ são linearmente independentes. Seja $a \in \mathbb{C}^{n}$ tal que $\operatorname{Gr}\left(v_{1}, \ldots, v_{n}\right) a=0$. Podemos escrever

$$
a=\sum_{k=1}^{n} a_{k} v_{k},
$$


com $a_{k} \in \mathbb{C}^{n}, k=1, \ldots, n$. Então, para todo $j=1,2, \ldots, n$,

$$
\left\langle v_{j}, a\right\rangle=\sum_{k=1}^{n} a_{k}\left\langle v_{j}, v_{k}\right\rangle=0
$$

$\operatorname{logo} a$ é ortogonal a si mesmo, isto é, $\langle a, a\rangle=0$. Portanto $a=0$ e concluímos assim que $\operatorname{Gr}\left(v_{1}, \ldots, v_{n}\right)$ é não singular.

\section{Recíproca da desigualdade de Hölder}

Seja $\Omega$ um aberto de $\mathbb{R}^{2}$. Se $f \in L^{p}(\Omega), 1<p<\infty$, e $g \in L^{q}(\Omega)$ com $1 / p+1 / q=1$, temos pela desigualdade de Hölder que $f g \in L^{1}(\Omega)$. A recíproca desse resultado segue do seguinte teorema:

Teorema 25. (Recíproca da desigualdade de Hölder) Seja $\Omega$ um aberto de $\mathbb{R}^{2}$. Seja $f \in L^{1}(\Omega$ ). Se tivermos $f g \in L^{1}(\Omega)$, para toda $g \in L^{p}(\Omega), 1<p<\infty$, então $f \in L^{q}(\Omega) \operatorname{com} 1 / p+1 / q=1$.

(Veja, (YEH, 2006); também, (LEACH, 1956))

\section{Teoremas de ponto fixo}

Teorema 26. (Teorema do Ponto Fixo de Schauder) Seja $X$ um espaço de Banach. Se $T: A \rightarrow$ $A$ é uma aplicação contínua, onde $A$ é um subconjunto não vazio, compacto e convexo de $X$, então $T$ possui um ponto fixo, i.e., existe $\mathrm{p} \in A$ tal que $T(\mathrm{p})=\mathrm{p}$.

Veja uma demonstração em (COURANT; HILBERT, 1962), na página 403.

Seja $X$ um espaço de Banach com a norma $\|\cdot\|_{X}$ e $\mathscr{B}_{X}=\left\{f \in X ;\|f\|_{X} \leq 1\right\}$. Dizemos que um operador linear contínuo $G: X \rightarrow X$ é compacto se $G\left(\mathscr{B}_{X}\right)$ é pré-compacto no topologia forte de $X$.

O nosso próximo teorema refere-se na resolubilidade da equação $(I-G) u=v$.

Teorema 27. (Alternativa de Fredholm). Seja X é um espaço de Banach e $G: X \rightarrow X$ um operador compacto. Temos que $\operatorname{Ker}(I-G)=0$ se, e somente se, $\operatorname{Im}(I-G)=X$.

Veja (BREZIS, 2010). 



\section{ALGUNS RESULTADOS DA TEORIA \\ CLÁSSICA}

Neste apêndice vamos falar um pouco da teoria clássica que trata de equações envolvendo o operador de Cauchy-Riemann

$$
\frac{\partial}{\partial \bar{z}}=\frac{1}{2}\left(\frac{\partial}{\partial x}+i \frac{\partial}{\partial y}\right), z=x+i y
$$

O conteúdo deste apêndice pode ser encontrado em (BEGEHR, 1994), (VEKUA, 1962) e (GAKHOV, 1966).

Seja $\Gamma$ uma curva suave por partes em $\mathbb{C}$ e $\varphi \in C(\Gamma)$. Então,

$$
\phi(z):=\frac{1}{2 \pi} \int_{\Gamma} \frac{\varphi(\zeta)}{\zeta-z} d \zeta
$$

é holomorfa em $\mathbb{C} \backslash \Gamma$ e $\phi(\infty)=0$. Em geral, $\phi$ não existe sobre a curva $\Gamma$.

Seja $\Gamma$ uma curva fechada simples e suave por partes em $\mathbb{C}$. Se $\varphi \in C^{\alpha}(\Gamma), 0<\alpha<1$, então $\phi$ dada por (B.1) existe sobre $\Gamma$ como valor principal de Cauchy.

O teorema a seguir é conhecido como fórmula de Plemelj-Sokhotzki.

Teorema 28. (PLEMELJ-SOKHOTZKI). Com as mesmas condições acima, temos que

$$
\phi^{+}\left(z_{0}\right):=\lim _{z \rightarrow z_{0}} \phi(z), z \in D^{+}, \text {e } \phi^{-}\left(z_{0}\right):=\lim _{z \rightarrow z_{0}} \phi(z), z \in D^{-}
$$

onde $D^{+}$é a região delimitada por $\Gamma=\partial D^{+}$e $D^{-}=\mathbb{C} \backslash \overline{D^{+}}$. Além disso, para $z_{0} \in \Gamma$,

$$
\phi^{+}\left(z_{0}\right)-\phi\left(z_{0}\right)^{-}=\varphi\left(z_{0}\right) \text { e } \phi^{+}\left(z_{0}\right)+\phi\left(z_{0}\right)^{-}=2 \varphi\left(z_{0}\right)
$$

Mais ainda, $\phi^{+} \in C^{\alpha}\left(\overline{D^{+}}\right)$e $\phi^{-} \in C^{\alpha}\left(\overline{D^{-}}\right)$. 
Seja $D \subset \mathbb{C}$ um aberto limitado.

Definição 14. Para $f \in L^{1}(D)$ definimos o operador integral

$$
T f(z)=-\frac{1}{\pi} \int_{D} \frac{f(\zeta)}{\zeta-z} d \xi d \eta, \zeta=\xi+i \eta, z \in \mathbb{C} .
$$

O operador $T f$ é conhecido como operador de Cauchy-Pompeiu.

Teorema 29. Se $f \in L^{p}(D)$, com $p>2$, então

$$
|T f(z)| \leq M(p, D)\|f\|_{p}, \text { para todo } z \in \mathbb{C}
$$

Demonstração. Seja $z \in D$. Pela desigualdade de Hölder,

$$
|T f(z)| \leq \frac{\|f\|_{p}}{\pi}\left(\int_{D} \frac{d \xi d \eta}{|\zeta-z|^{q}}\right)^{\frac{1}{q}}=: \frac{\|f\|_{p}}{\pi} I^{\frac{1}{q}}, q=p /(p-1) .
$$

Seja $\mathrm{d}=\operatorname{diam}(D)=\sup \{|\zeta-z| ; \zeta, z \in D\}$. Temos assim,

$$
I=\int_{D} \frac{d \xi d \eta}{|\zeta-z|^{q}} \leq \int_{|\zeta-z| \leq \mathrm{d}} \frac{d \xi d \eta}{|\zeta-z|^{q}}=2 \pi \int_{0}^{\mathrm{d}} r^{1-q} d r=\frac{2 \pi}{2-q} \mathrm{~d}^{2-q} .
$$

Portanto, tomando $M(p, D)=\frac{1}{\pi}\left(\frac{2 \pi}{2-q} \mathrm{~d}^{2-q}\right)^{\frac{1}{q}}$ temos o resultado.

Sejam $\alpha, \beta \in \mathbb{R}$ com $0<\alpha<2$ e $0<\beta<2$. Para $z_{1}, z_{2} \in \mathbb{C}$, com $z_{1} \neq z_{2}$, definimos

$$
J_{\alpha, \beta}\left(z_{1}, z_{2}, D\right)=\int_{D} \frac{d \xi d \eta}{\left|\zeta-z_{1}\right|^{\alpha}\left|\zeta-z_{2}\right|^{\beta}}, \zeta=\xi+i \eta
$$

Lema 18. (Desigualdade de Hadamard) Existem constantes $M_{1}(\alpha, \beta), M_{2}(\alpha, \beta, D)$ e $M_{3}(\alpha, \beta, D)>$ 0 tais que

$$
J_{\alpha, \beta}\left(z_{1}, z_{2}, D\right) \leq \begin{cases}M_{1}(\alpha, \beta)\left|z_{1}-z_{2}\right|^{2-\alpha-\beta}, & \text { se } \alpha+\beta>2 \\ M_{2}(\alpha, \beta, D)+8 \pi|\ln | z_{1}-z_{2}||, & \text { se } \alpha+\beta=2 \\ M_{3}(\alpha, \beta, D), & \text { se } \alpha+\beta<2\end{cases}
$$

Demonstração. Seja $\rho>0$ tal que

$$
\bar{D} \subset\left\{z \in \mathbb{C} ;\left|z-z_{1}\right|<2 \rho\right\} .
$$

Sejam

$$
D_{1}=\left\{z \in \mathbb{C} ;\left|\zeta-z_{1}\right|<2 \rho\right\} \text { e } D_{2}=\left\{z \in \mathbb{C} ;\left|z-z_{1}\right|<2\left|z_{1}-z_{2}\right|\right\}
$$

Temos

$$
J_{\alpha, \beta}\left(z_{1}, z_{2}, D\right) \leq \int_{D_{1} \backslash D_{2}} \frac{d \xi d \eta}{\left|\zeta-z_{1}\right|^{\alpha}\left|\zeta-z_{2}\right|^{\beta}}+\int_{D_{2}} \frac{d \xi d \eta}{\left|\zeta-z_{1}\right|^{\alpha}\left|\zeta-z_{2}\right|^{\beta}}:=J_{1}+J_{2} .
$$


Para $\zeta \in D_{1} \backslash D_{2}$, temos

$$
2\left|z_{1}-z_{2}\right| \leq\left|\zeta-z_{1}\right|<2 \rho
$$

Daí,

$$
\left|\zeta-z_{2}\right| \geq\left|\zeta-z_{1}\right|-\left|z_{1}-z_{2}\right| \geq\left|\zeta-z_{1}\right|-\frac{1}{2}\left|\zeta-z_{1}\right|=\frac{1}{2}\left|\zeta-z_{1}\right|
$$

Assim,

$$
J_{1} \leq 2^{\beta} \int_{D_{1} \backslash D_{2}} \frac{d \xi d \eta}{\left|\zeta-z_{1}\right|^{\alpha+\beta}}=2^{\beta+1} \pi \int_{2\left|z_{1}-z_{2}\right|}^{2 \rho} \frac{d r}{r^{\alpha+\beta-1}}
$$

Logo,

$$
J_{1} \leq 2^{\beta+1} \pi \begin{cases}\frac{2^{2-\alpha-\beta}}{\alpha+\beta-2}\left|z_{1}-z_{2}\right|^{2-\alpha-\beta}, & \text { se } \alpha+\beta>2, \\ \ln \frac{\rho}{\left|z_{1}-z_{2}\right|}, & \text { se } \alpha+\beta=2, \\ \frac{2^{2-\alpha-\beta}}{2-\alpha-\beta} \rho^{2-\alpha-\beta}, & \text { se } \alpha+\beta<2 .\end{cases}
$$

Observe,

$$
\ln \frac{\rho}{\left|z_{1}-z_{2}\right|}=\ln (\rho)-\ln \left|z_{1}-z_{2}\right| \leq|\ln (\rho)|+|\ln | z_{1}-z_{2}|| .
$$

Estimando $J_{2}$ temos

$$
J_{2}=\int_{D_{2}} \frac{d \xi d \eta}{\left|\zeta-z_{1}\right|^{\alpha}\left|\zeta-z_{2}\right|^{\beta}}=\left|z_{1}-z_{2}\right|^{-\alpha-\beta} \int_{D_{2}} \frac{d \xi d \eta}{\left|\frac{\zeta-z_{1}}{z_{1}-z_{2}}\right|^{\alpha}\left|\frac{\zeta-z_{2}}{z_{1}-z_{2}}\right|^{\beta}} .
$$

Temos que

Assim

$$
\left|\frac{\zeta-z_{2}}{z_{1}-z_{2}}\right|=\left|\frac{\zeta-z_{1}}{z_{1}-z_{2}}+\frac{z_{1}-z_{2}}{z_{1}-z_{2}}\right|=\left|\frac{\zeta-z_{1}}{z_{1}-z_{2}}+1\right| .
$$

$$
J_{2}=\left|z_{1}-z_{2}\right|^{-\alpha-\beta} \int_{D_{2}} \frac{d \xi d \eta}{\left|\frac{\zeta-z_{1}}{z_{1}-z_{2}}\right|^{\alpha}\left|\frac{\zeta-z_{1}}{z_{1}-z_{2}}+1\right|^{\beta}} .
$$

Mudando de coordenada através da função $F: D_{2} \rightarrow B(0,2)$ dada por

$$
F(\zeta)=\frac{\zeta-z_{1}}{z_{1}-z_{2}}
$$

temos

$$
J_{2}=\left|z_{1}-z_{2}\right|^{2-\alpha-\beta} \int_{B(0,2)} \frac{d \xi d \eta}{|\zeta|^{\alpha}|\zeta+1|^{\beta}} \leq M(\alpha, \beta)\left|z_{1}-z_{2}\right|^{2-\alpha-\beta}
$$

onde $M(\alpha, \beta)$ é uma constante positiva.

Juntando as estimativas de $J_{1}$ e $J_{2}$ obtemos a estimativa (B.3).

Teorema 30. Se $f \in L^{p}(D)$, com $p>2$, então

$$
\left|T f\left(z_{1}\right)-T f\left(z_{2}\right)\right| \leq C(p, D)|| f||_{p}\left|z_{1}-z_{2}\right|^{\frac{2-q}{q}}, \text { para todos } z_{1}, z_{2} \in \mathbb{C},
$$

$\operatorname{com} q=p /(p-1)$. 
Demonstração. Sejam $z_{1}, z_{2} \in \mathbb{C}, \operatorname{com} z_{1} \neq z_{2}$. Temos, pela desigualdade de Hölder,

$$
\begin{aligned}
& \left|T f\left(z_{1}\right)-T f\left(z_{2}\right)\right|=\left|\frac{\left(z_{1}-z_{2}\right)}{\pi} \int_{D} \frac{f(\zeta)}{\left(\zeta-z_{1}\right)\left(\zeta-z_{2}\right)} d \xi d \eta\right| \\
\leq & \frac{\left|z_{1}-z_{2}\right|}{\pi}|| f\left\|_{p}\left(\int_{D} \frac{d \xi d \eta}{\left|\zeta-z_{1}\right|^{q}\left|\zeta-z_{2}\right|^{q}}\right)^{\frac{1}{q}}=: \frac{\left|z_{1}-z_{2}\right|}{\pi}|| f\right\|_{p} J^{\frac{1}{q}} .
\end{aligned}
$$

Pela Desigualdade de Hadamard, lema 18, temos

$$
J \leq M(q)\left|z_{1}-z_{2}\right|^{2-q} .
$$

Daí,

$$
\left|T f\left(z_{1}\right)-T f\left(z_{2}\right)\right| \leq \frac{\left|z_{1}-z_{2}\right|}{\pi}|| f||_{p}\left(M(q)\left|z_{1}-z_{2}\right|^{2-2 q}\right)^{\frac{1}{q}}=C(q)|| f||_{p}\left|z_{1}-z_{2}\right|^{\frac{2-q}{q}} .
$$

Observação 30. Dizer que $\Gamma \subset \mathbb{C}$ é uma curva de classe $C^{1+\varepsilon}, 0 \leq \varepsilon<1$, equivale a dizer que $\tilde{G}=\left\{(x, y) \in \mathbb{R}^{2} ; x+i y \in \Gamma\right\} \subset \mathbb{R}^{2}$ é uma subvariedade mergulhada de dimensão 1 , de classe $C^{1+\varepsilon}$, de $\mathbb{R}^{2}$. Veja em (MUSKHELISHVILI, 1953) sobre propriedades de curvas suaves. Sobre a teoria de variedades diferenciáveis veja (LIMA, 2007).

Teorema 31. (Fórmula de representação Cauchy-Pompeiu). Seja $U \subset \mathbb{C}$ um aberto limitado simplesmente conexo com bordo de classe $C^{1}$. Se $w$ é uma função de classe $C^{1}$ numa vizinhança de $\bar{U}$, então

$$
w(z)=\frac{1}{2 \pi i} \int_{\partial U} \frac{w(\zeta)}{\zeta-z} d \zeta-\frac{1}{\pi} \int_{U} \frac{\partial w}{\partial \bar{z}}(\zeta) \frac{d \xi d \eta}{\zeta-z}, \zeta=\xi+i \eta
$$

para todo $z \in U$.

Observação 31. A expressão "função de classe $C^{1}$ numa vizinhança de $\bar{U}$ " no enunciado acima significa que $\tilde{w}$ é uma função de classe $C^{1}$, onde $\tilde{w}(x, y)=w(x+i y)$, numa vizinhança de $\left\{(x, y) \in \mathbb{R}^{2} ; x+i y \in \bar{U}\right\}$ em $\mathbb{R}^{2}$.

Observação 32. A Fórmula de representação Cauchy-Pompeiu também é conhecida como a Fórmula de Cauchy não-homogênea.

O operador $T$ pode ser usado para obter soluções fracas para a equação $\partial w / \partial \bar{z}=f$ em $D$, para $f$ com "baixa regularidade" como veremos no próximo teorema. A demonstração é uma consequência do Teorema 31. Veja (BEGEHR, 1994) e (VEKUA, 1962).

Teorema 32. Se $f \in L^{1}(D)$, então $\frac{\partial T f}{\partial \bar{z}}=f$ em $D$. 
Seja $D$ um aberto simplesmente conexo $\mathbb{C}$ com bordo de classe $C^{1}$ e com $0 \in D$.

Definição 15. O problema de contorno de Schwarz consiste em encontrar uma função holomorfa $w$ em $D$, i.e., $\partial w / \partial \bar{z}=0$ em $D$, que satisfaz

$$
\mathfrak{R}(w)=\gamma \operatorname{em} \partial D \text { e } \mathfrak{I}(w)(0)=c
$$

para $\gamma \in C(\partial D ; \mathbb{R}), c \in \mathbb{R}$ dados.

Definição 16. Um operador

$$
S: C(\partial D ; \mathbb{R}) \rightarrow \mathscr{A}(D) \cap C(\bar{D} ; \mathbb{C})
$$

do espaço das funções contínuas a valores reais definidas em $D$ sobre o espaço das funções holomorfas em $D, \mathscr{A}(D)$, que são contínuas em $\bar{D}$ que satisfaz

$$
\mathfrak{R}(S \varphi)=\varphi \operatorname{em} \partial D
$$

é chamado de operador de Schwarz.

$$
\text { Seja } \mathbb{D}=\{z \in \mathbb{C} ;|z|<1\} .
$$

Proposição 25. No caso $D=\mathbb{D}$ o operador de Schwarz com o núcleo de Schwarz-Poisson é

$$
(S \varphi)(z)=\frac{1}{2 \pi i} \int_{|\zeta|=1} \varphi(\zeta) \frac{\zeta+z}{\zeta-z} \frac{d \zeta}{\zeta}+i c, \quad z \in \overline{\mathbb{D}}
$$

onde $c \in \mathbb{R}$ é uma constante arbitrária.

Observação 33. O operador definido na proposição acima satisfaz $\mathfrak{I}\{S \varphi\}(0)=c$.

Teorema 33. (PRIVALOV). Seja $w=u+i v$ uma função holomorfa em $\mathbb{D}$, onde $v$ é contínua em $\overline{\mathbb{D}}$ e Hölder contínua sobre o bordo $\partial \mathbb{D}$, satisfazendo, para $0<\alpha<1$,

$$
\left|v\left(z_{1}\right)-v\left(z_{2}\right)\right| \leq K\left|z_{1}-z_{2}\right|^{\alpha}, \zeta_{1}, \zeta_{2} \in \partial \mathbb{D}
$$

Então, $w$ é Hölder contínua em $\overline{\mathbb{D}}$ com o mesmo expoente e constante $c K, c=c(\alpha)$, i.e.,

$$
\left|w\left(z_{1}\right)-w\left(z_{2}\right)\right| \leq c K\left|z_{1}-z_{2}\right|^{\alpha}, \zeta_{1}, \zeta_{2} \in \overline{\mathbb{D}}
$$

(Veja (BEGEHR, 1994)) 
Temos assim o seguinte corolário.

Corolário 9. Se $g \in C^{\alpha}(\partial \mathbb{D} ; \mathbb{R}), 0<\alpha<1$, então $S g \in C^{\alpha}(\overline{\mathbb{D}})$.

Demonstração. Segue direto da Proposição 25 e do Teorema 33.

O Problema de Riemann-Hilbert. Dados $\Lambda \in C^{\alpha}(\partial D)$, com $|\Lambda|=1$ em $\partial D, \varphi \in C^{\alpha}(\partial D, \mathbb{R})$, $0<\alpha<1$, o Problema de Riemann-Hilbert consiste em encontrar uma função $w$ holomorfa em $D$, Hölder contínua em $\bar{D}$, e tal que

$$
\Re(\overline{\Lambda(\zeta)} w(\zeta))=\varphi(\zeta) \text { para } \zeta \in \partial D
$$

Observação 34. Em alguns livros a condição de fronteira (B.5) é escrita de uma forma diferente. Se escrevermos $w$ e $\Lambda$ em termos de suas partes reais e imaginas, $w=w_{1}+i w_{2}$ e $\Lambda=\Lambda_{1}+i \Lambda_{2}$, então podemos escrever (B.5) como

$$
\Lambda_{1} w_{1}+\Lambda_{2} w_{2}=\varphi \operatorname{em} \partial D
$$

Seja $\Gamma$ uma curva fechada simples de classe $C^{1}$ em $\mathbb{C}$

Definição 17. Seja $G \in C(\Gamma, \mathbb{C}),|G|=1$. O índice de $G$ com respeito a $\Gamma$ é definido pelo número inteiro

$$
\operatorname{ind}_{\Gamma}(G)=\frac{1}{2 \pi} \int_{\Gamma} d \arg (G) .
$$

Observação 35. A integral acima é entendida no sentido da integral de Stieltjes. Veja (GAKHOV, 1966) e (BEGEHR, 1994) sobre a definição do índice e propriedades. Veja também a seção 2.4, na página 38.

A partir do operador de Schwarz podemos obter a solução do problema de RiemannHilbert. No próximo teorema, o aberto $D$ será o disco aberto $\mathbb{D}$, e $S$ denotará o operador em (B.4).

Teorema 34. (BEGEHR, 1994) O problema de Riemann-Hilbert para o índice $\kappa=\operatorname{ind}_{\partial \mathbb{D}}(\Lambda) \geq 0$ é resolúvel. A solução geral é dada por

$$
w(z)=z^{\kappa} e^{i \gamma(z)}\left[(S \tilde{\varphi})(z)+i c_{0}+\sum_{k=1}^{\kappa}\left(c_{k} z^{k}-\overline{c_{k}} z^{-k}\right)\right], z \in \mathbb{D}
$$

onde

$$
\begin{aligned}
\gamma(z) & :=\left(\operatorname{Sarg}\left(\zeta^{-\kappa} \Lambda(\zeta)\right)\right)(z), z \in \mathbb{D} \\
\tilde{\varphi}(\zeta) & :=e^{\mathfrak{I}(\gamma(\zeta))}|\zeta|^{-\kappa} \varphi(\zeta), \zeta \in \partial \mathbb{D}
\end{aligned}
$$

e $c_{0} \in \mathbb{R}, c_{k} \in \mathbb{C}, 1 \leq k \leq \kappa$, são constantes arbitrarias. 
Observação 36. $\mathrm{O}$ caso em que $\kappa=\operatorname{ind}_{\partial \mathbb{D}}(\Lambda)<0$ o problema de Riemann-Hilbert possui solução se e somente se $S \tilde{\varphi}$ possui um zero de ordem $-\kappa$ em $0 \in \mathbb{D}$. Usando a expressão do operador de Schwarz de $\mathbb{D}$ (dado em (B.4)) podemos mostrar que $S \tilde{\varphi}$ ter um zero de ordem $-\kappa$ em $0 \in \mathbb{D}$ equivale as $-\kappa$ condições de compatibilidade

$$
\int_{|\zeta|=1} \tilde{\varphi}(\zeta) \frac{d \zeta}{\zeta^{k+1}}, 0 \leq k \leq-\kappa-1 .
$$

Neste caso, a solução fica da forma $w(z)=z^{\kappa} e^{i \gamma(z)}(S \tilde{\varphi})(z), z \in \overline{\mathbb{D}}$. Veja (BEGEHR, 1994).

Vamos supor que $D$ tenha bordo suave de classe $C^{1+\varepsilon}, 0<\varepsilon<1$. Utilizando o Teorema de Riemann 21 (página 131) podemos obter um operador de Schwarz para o aberto simplesmente conexo $D$ e também uma solução do problema de Riemann-Hilbert.

De fato, seja $\Phi: \bar{D} \rightarrow \overline{\mathbb{D}}$ um difeomorfismo de classe $C^{1+\varepsilon}$ tal que $\partial \Phi / \partial \bar{z}=0$ em $D$ e $\Phi(\partial D)=\partial \mathbb{D}($ veja Teorema 21$)$. Temos que $\mathscr{S}: C(\partial D) \rightarrow \mathscr{A}(D) \cap C(\bar{D} ; \mathbb{C})$ dado por

$$
\mathscr{S} \varphi:=\left(S\left(\varphi \circ \Phi^{-1}\right)\right)(\Phi)
$$

é um operador de Schwarz para $D$ (definição 16). Assim, o seguinte corolário está provado:

Corolário 10. (Operador de Schwarz para $D$ ) Seja $D$ um aberto limitado simplesmente conexo de $\mathbb{C}$ com bordo suave de classe $C^{1+\varepsilon}, 0<\varepsilon<1$. Então existe um operador $\mathscr{S}: C(\partial D) \rightarrow$ $\mathscr{A}(D) \cap C(\bar{D} ; \mathbb{C})$ tal que

$$
\Re(\mathscr{S} \varphi)=\varphi \text { em } \partial D, \text { para todo } \varphi \in C(\partial D)
$$

Além disso, se $\varphi \in C^{\alpha}(\partial D), 0<\alpha<1$, então $\mathscr{S} \varphi \in C^{\alpha}(\bar{D})$.

Da mesma forma podemos provar uma versão do Teorema 34 usando o difeomorfismo $\Phi: \bar{D} \rightarrow \overline{\mathbb{D}}$ (descrito no paragrafo antes do Teorema 10).

Corolário 11. Seja $D$ um aberto limitado simplesmente conexo de $\mathbb{C}$ com bordo suave de classe $C^{1+\varepsilon}, 0<\varepsilon<1$. Sejam $\Lambda \in C^{\alpha}(\partial D)$, com $|\Lambda|=1$ em $\partial D, \varphi \in C^{\alpha}(\partial D, \mathbb{R}), 0<\alpha<1$. Se $\operatorname{ind}_{\partial D}(\Lambda) \geq 0$, então existe uma função $w$ holomorfa em $D$, com $w \in C^{\alpha}(\bar{D})$, tal que

$$
\Re(\overline{\Lambda(\zeta)} w(\zeta))=\varphi(\zeta) \text { para } \zeta \in \partial D
$$

Demonstração. Vamos usar a mesma notação usada no paragrafo anterior ao Corolário 10. Sejam $\tilde{\Lambda}:=\Lambda \circ \Phi^{-1}$ e $\tilde{\varphi}:=\varphi \circ \Phi^{-1}$. Como $\Phi: \bar{D} \rightarrow \overline{\mathbb{D}}$ é um difeomorfismo de classe $C^{1+\varepsilon}$, temos que $\tilde{\Lambda} \in C^{\alpha}(\partial \mathbb{D})$ e $\tilde{\varphi}:=\varphi \circ \Phi^{-1} \in C^{\alpha}(\partial \mathbb{D}, \mathbb{R})$. Além disso, como $\Phi$ é uma função 
holomorfa em $D$ e $D$ é simplesmente conexo, segue que $\Phi$ preserva orientação. Em particular $\operatorname{ind}_{\partial \mathbb{D}}(\tilde{\Lambda})=\operatorname{ind}_{\partial D}(\Lambda) \geq 0$. Pelo Teorema 34 existe $h$ solução do problema

$$
\left\{\begin{array}{l}
\frac{\partial h}{\partial \bar{z}}=0 \quad \text { em } \quad \mathbb{D} \\
\Re(\bar{\Lambda} h)=\tilde{\varphi} \quad \text { em } \quad \partial \mathbb{D} .
\end{array}\right.
$$

Então, $w:=h \circ \Phi$ é de classe $C^{\alpha}(\bar{D})$, holomorfa em $D$ e satisfaz a condição de bordo (B.6).

Usando um fator integrante obtido através da função exponencial e da resolubilidade global do operador de Cauchy-Riemann (Teorema 18, página 126) podemos provar um resultado um pouco mais geral do que o corolário acima.

Corolário 12. Seja $D$ um aberto limitado simplesmente conexo de $\mathbb{C}$ com bordo suave de classe $C^{1+\varepsilon}, 0<\varepsilon<1$. Sejam $\Lambda \in C^{\alpha}(\partial D)$, com $|\Lambda|=1 \mathrm{em} \partial D, \varphi \in C^{\alpha}(\partial D, \mathbb{R}), 0<\alpha<1$. Sejam $a$, $f$ funções de classe $C^{\infty}$ numa vizinhança de $\bar{D}$. Se ind ${ }_{\partial D}(\Lambda) \geq 0$, então o problema de contorno

$$
\left\{\begin{array}{l}
\frac{\partial w}{\partial \bar{z}}=a w+f \quad \text { em } \quad D \\
\Re(\bar{\Lambda} w)=\varphi \quad \text { em } \quad \partial D
\end{array}\right.
$$

possui uma solução Hölder contínua em $\bar{D}$ e suave em $D$.

Observação 37. O termo suave no enunciado acima significa que $\tilde{w} \in C^{\infty}(\tilde{D})$, onde $\tilde{w}(x, y)=$ $w(x+i y)$ e $\tilde{D}=\left\{(x, y) \in \mathbb{R}^{2} ; x+i y \in D\right\}$.

Demonstração. (do Corolário 12). A demonstração é análoga ao início da demonstração do Teorema 20 (página 128) trocando $L$ por $\partial / \partial \bar{z}$.

O Problema de Riemann-Hilbert Generalizado. O problema de contorno que busca uma função $w \in C(\bar{D})$ que seja solução da equação

$$
\frac{\partial w}{\partial \bar{z}}=a w+b \bar{w}+f \text { em } D
$$

onde $a, b$ e $f \in L^{p}(D)$, e que satisfaça a condição de contorno

$$
\mathfrak{R}(\bar{\Lambda} w)=\varphi \operatorname{em} \partial D
$$

com $\Lambda$ e $\varphi$ como no Problema de Riemann-Hilbert (página 140) é chamado de Problema de Riemann-Hilbert generalizado e é um dos temas centrais de estudo de (VEKUA, 1962). Em (VEKUA, 1962) é provado que o problema acima possui solução Hölder contínua em $\bar{D}$ quando $a, b$ e $f \in L^{p}(D)$, com $p>2$ e $\operatorname{ind}_{\partial D}(\Lambda) \geq 0$. A demonstração desse resultado usa elementos 
da Análise Funcional e Topologia para obter a solução do problema acima através de ponto fixo de operadores integrais. $O$ caso em que ind ${ }_{\partial D}(\Lambda)<0$ depende da análise de condições de compatibilidade (do mesmo tipo que as mencionadas na observação 36 quando $D=\mathbb{D}$ ). 



\section{REFERÊNCIAS}

ABLOWITZ, M.; CLARKSON, P. Solitons, Nonlinear Evolution Equations and Inverse Scattering. [S.1.: s.n.], 1991. (London Mathematical Society Lecture Note Series 149, Cambridge University Press). Citado na página 17.

AHLFORS, L.; SARIO, L. Riemann Surfaces. [S.1.: s.n.], 1960. (Princeton Math. Series, vol. 2, Princeton University Press, Princeton). Citado 2 vezes nas páginas 38 e 39.

BEGEHR, H. Complex analytic methods for partial differential equations. An introductory text. [S.1.: s.n.], 1994. Citado 15 vezes nas páginas 19, 23, 39, 77, 89, 104, 107, 108, 114, 129, $135,138,139,140$ e 141.

BEGEHR, H.; CELEBI, A.; TUTSCHKE, W. Complex methods for partial differential equations. 1999. Citado na página 17.

BEGEHR, H.; GILBERT, R.; WEN, G. Partial differential and integral equations. International Society for Analysis, Applications and Computations, Kluwer Academic Publishers, 1999. Citado na página 17.

BERGAMASCO, A.; CORDARO, P.; HOUNIE, J. Global properties of a class of vector fields in the plane. Journal of differential equations, v. 74, p. 179-199, 1988. Citado 2 vezes nas páginas 36 e 57.

BERHANU, S.; CORDARO, P.; HOUNIE, J. An introduction to involutive structures. [S.1.: s.n.], 2008. (New Math. Mono., 6, Cambridge University Press, Cambridge). Citado 6 vezes nas páginas $17,18,35,57,58$ e 126.

BERHANU, S.; HOUNIE, J.; SANTIAGO, P. A similarity principle for complex vector fields and applications. Trans. Amer. Math. Soc. 353, no. 4, p. 1661-1675, 2001. Citado 2 vezes nas páginas 92 e 95.

BERS, L. An outline of the theory of pseudoanalytic functions. v. 62, p. 291-331, 1956. Citado na página 89.

BREZIS, H. Functional analysis, Sobolev spaces and partial differential equations. [S.1.: s.n.], 2010. Citado na página 133.

CAMPANA, C. Campos Hipoelíticos no Plano. [S.l.: s.n.], 2013. (Dissertação ( Mestrado em Matemática)-Instituto de Ciências Matemáticas e de Computação, Universidade de São Paulo, São Carlos, abril de 2013). Citado 2 vezes nas páginas 32 e 57.

CAMPANA, C.; MEZIANI, A. Boundary value problems for a class of planar complex vector fields. Jornal of Differential Equations, 10, v. 261, p. 5609-5636, 2016. Citado 3 vezes nas páginas 18,115 e 118.

CAMPANA, C.; SILVA, P. L. Dattori da; MEZIANI, A. Riemann-hilbert problem for a class of hypocomplex vector fields. Complex Variables and Elliptic Equations, 12, v. 61, p. 16561667, 2016. Citado 2 vezes nas páginas 18 e 109. 
Properties of solutions of a class of hypocomplex vector fields. Contemporary Mathematics, v. 681, p. 29-50, 2017. Citado 5 vezes nas páginas 18, 53, 62, 64 e 95.

CHEN, Y. Single crack problem with polynomial traction on the crack. Engineering Fracture Mechanics, v. 46, p. 353-355, 1993. Citado na página 17.

COURANT, R.; HILBERT, D. Methods of mathematical physics, vol. II. [S.1.: s.n.], 1962. Citado 3 vezes nas páginas 32, 89 e 133.

FARKAS, H. M.; KRA, I. Riemann Surfaces. [S.1.: s.n.], 1980. (Springer-Verlag, New York). Citado na página 131.

GAKHOV, F. D. Boundary Value Problems. [S.1.: s.n.], 1966. (Dover Publications, Inc.). Citado 8 vezes nas páginas 18, 19, 23, 39, 108, 114, 135 e 140.

HOUNIE, J. Teoria Elementar das Distribuições. [S.l.: s.n.], 1979. (Publicações do IMPA, $12^{\circ}$ Colóquio Brasileiro de Matemática, IMPA - Rio de Janeiro). Citado na página 33.

HöRMANDER, L. The Analysis of Linear Partial Differential Operators I, PseudoDifferential Operators. [S.1.: s.n.]. v. 1. Citado 3 vezes nas páginas 28, 29 e 91.

The Analysis of Linear Partial Differential Operators III, Pseudo-Differential Operators. [S.1.: s.n.]. v. 3. Citado na página 91.

An introduction to complex analysis in several variables. [S.1.: s.n.], 1973. (Fundamenta Mathematicae). Citado na página 126.

KRANTZ, S. G. Geometric Function Theory: Explorations in Complex Analysis. [S.1.: s.n.], 2006. (Springer Science \& Business Media). Citado na página 131.

LEACH, E. B. On a converse of the hölder inequality. Proc. Amer. Math. Soc. 7, p. 607-608, 1956. Citado na página 133.

LIMA, E. L. Grupo Fundamental e Espaços de Recobrimeno. [S.1.: s.n.], 2006. (Projeto Euclides, Rio de Janeiro, IMPA, terceira edição). Citado na página 38.

Variedades Diferenciáveis. [S.1.: s.n.], 2007. (Publicações Matemáticas, Rio de Janeiro, IMPA). Citado 3 vezes nas páginas 26, 45 e 138.

Curso de Análise. [S.1.: s.n.], 2008. v. 2. (Projeto Euclides, Rio de Janeiro, IMPA, décima edição, v. 2). Citado na página 76.

LINS-NETO, A. Funções de uma variável complexa. [S.1.: s.n.], 2005. (Projeto Euclides, Rio de Janeiro, IMPA, segunda edição). Citado na página 76.

MEZIANI, A. On the similarity principle for planar vector fields: application to second order pde. Jornal of Differential Equations 157, no. 1, p. 1-19, 1999. Citado na página 95.

Representation of solutions of planar elliptic vector fields with degeneracies. Geometric analysis of PDE and several complex variables, 357-370, Contemp. Math., 368, Amer. Math. Soc., Providence, RI, 2005., 2005. Citado 4 vezes nas páginas 59, 62, 95 e 96.

Representation of solutions of a singular cauchy-riemann equation in the plane. v. 53, p. 1111-1130, 2008. Citado na página 72. 
Global solvability of real analytic complex vector fields in two variables. Jornal of Differential Equations, 251, p. 2896-2931, 2011. Citado 2 vezes nas páginas 18 e 44.

On first and second order planar elliptic equations with degeneracies. Mem. Amer. Math. Soc. 217, no. 1019, 2012. Citado na página 72.

MUSKHELISHVILI, N. I. Singular Integral Equations, Boundary problems of functions theoty and their application to mathematical physics. [S.1.: s.n.], 1953. (Groningen-Hollandl). Citado 3 vezes nas páginas 17, 18 e 138.

POMMERENKE, C. Boundary Behaviour of Conformal Maps. [S.1.: s.n.], 1992. (SpringerVerlag). Citado na página 131.

SPRINGER, G. Introduction to Riemann Surfaces. [S.1.: s.n.], 1957. (Addison-Wesley, MA). Citado na página 131.

TREVES, F. Hypoelliptic partial differential equations of principal type. sufficient conditions and necessary conditions*. Comm. Pure Appl. Math. 24, p. 631-670, 1971. Citado 3 vezes nas páginas 42, 57 e 126.

Hypo-analytic structures: Local theory. 1992. Citado 3 vezes nas páginas 17, 35 e 57.

VEKUA, I. N. Generalized Analytic Functions. [S.1.: s.n.], 1962. v. 25. (Pergamon Press, v. 25). Citado 14 vezes nas páginas 17, 19, 23, 77, 89, 104, 107, 108, 114, 118, 129, 135, 138 e 142.

YEH, J. Real analysis. Theory of measure and integration, Second edition. [S.l.: s.n.], 2006. (World Scientific Publishing, NJ). Citado na página 133.

ZALCMAN, L. Real proofs of complex theorems (and vice versa). Mathematical Association of America; The American Mathematical Monthly, No. 2, v. 81, p. 115-137, 1974. Citado na página 132. 

Analítica, 27

Arzelà-Ascoli

teorema de, 86

Beltrami

equação de, 32

Biholomorfismo, 27

Cauchy

teorema de, 117

Cauchy-Pompeiu

Fórmula de representação de, 77, 138

operador de, 136

Cauchy-Riemann

operador de, 30

Condição (P), 57, 126

Condição (Q), 57

Conjunto característico, 30

Curva de classe $C^{1+\varepsilon}, 26,138$

Desigualdade de Hölder, 27

Desigualdade de Hadamard, 83, 136

Distribuições, 28

Elítico, 30

Fórmula de Aproximação de Baouendi-Treves, 57

Fredholm, 119, 133 teorema de, 119

Friedrichs

lema de, 91

Funções analíticas generalizadas, 89

Funções pseudo-analíticas, 89

Globalmente resolúvel, 126
Gramm

matriz de, 122, 132

Green

teorema de, 76

Hamiltoniano, 33

Hipocomplexo, 35

Hipoelítico, 57

Holomorfa, 27, 131

Integral primeira, 31

Lipschitz, 26

Localmente integrável, 31

Localmente resolúvel, 126

Mudança de coordenadas, 32

Mudança de variáveis, 31

Plemelj-Sokhotzki

fórmula de, 135

Princípio da Similaridade, 89

Princípio da similaridade, 121

Privalov, 139

Problema de Riemann-Hilbert, 140

Problema de Riemann-Hilbert Generalizado, 142

Riemann

superfície de, 37

Teorema da aplicação de, 131

teorema de uniformização de, 37

Schauder

Teorema do Ponto Fixo de, 87, 89, 111, 113,133

Schwarz 
teorema de, 49

O problema de contorno de, 139

Operador de, 107, 139

Schwarz-Poisson, 139

Tipo finito, 37, 58

Tipo infinito, 38, 52

Transversal, 44 